Supporting information

\title{
Stereoselective Synthesis of Terminal Monofluoroalkenes from Trifluoromethylated Alkenes
}

\author{
Pauline Poutrel, ${ }^{\dagger}$ Xavier Pannecoucke, ${ }^{\dagger}$ Philippe Jubault, ${ }^{+*}$ and Thomas Poisson ${ }^{\dagger, \neq *}$ \\ + Normandie Univ, INSA Rouen, UNIROUEN, CNRS, COBRA (UMR 6014), 76000 Rouen, France. \\ ${ }^{\ddagger}$ Institut Universitaire de France, 1 rue Descartes, 75231 Paris, France.
}




\title{
Supporting information
}

\author{
Table of contents
}

1. General information 3

2. Synthesis of starting materials 4

2.i. General procedure for the synthesis of $\alpha-\mathrm{CF}_{3}$ styrenes 4

2.ii. Characterization of $\alpha-\mathrm{CF}_{3}$ styrenes 4

2.iii. Preparation and characterization of compounds $1 p$ and $1 q$

2.iv. Preparation and characterization of compounds 1v, 1w and 1x 7

2.v. General procedure for the synthesis of $\beta-\mathrm{CF}_{3}$ acrylates 9

2.vi. Characterization of $\beta-\mathrm{CF}_{3}$ acrylates 9

3. Optimization \& mechanistic studies 11

$\begin{array}{ll}\text { 3.i. Optimization } & 11\end{array}$

$\begin{array}{ll}\text { 3.ii. Mechanistic studies } & 12\end{array}$

4. Determination of the stereochemistry of the monofluoroalkenes 15

5. Reduction of $\beta-\mathrm{CF}_{3}$ nitrostyrene and acrylonitrile 17

6. Synthesis of monofluoroalkenes $\quad 18$

$\begin{array}{lc}\text { 6.i. General procedures } & 18\end{array}$

$\begin{array}{lr}\text { 6.ii. Characterization } & 20\end{array}$

$\begin{array}{ll}\text { 7. References } & 33\end{array}$

8. NMR spectra $\quad 34$ 


\section{General information}

All reactions were carried out using oven dried glassware and magnetic stirring under argon atmosphere unless otherwise stated. When needed, reactions were heated with an oil bath. Tetrahydrofuran was distilled from sodium and benzophenone and dichloromethane was distilled from calcium hydride. Lithium aluminum hydride $95 \%$ in powder was purchased from Fisher Scientific and was stored in a glovebox. Other solvents and commercially available reagents were purchased from standard chemical suppliers (Sigma Aldrich, Fisher Scientific, VWR and Fluorochem) and used as received without further purification, unless otherwise stated.

Column chromatographies were carried out using silica gel $(40-63 \mu \mathrm{m})$ supplied by VWR and analytical thin layer chromatographies performed on pre-coated silica gel aluminium plates (60 $\mathrm{F}_{254}$ from Merck) were visualized by UV light $\left(254 \mathrm{~nm}\right.$ ) and/or stained with $\mathrm{KMnO}_{4}$.

${ }^{1} \mathrm{H}(300 \mathrm{MHz}),{ }^{13} \mathrm{C}(75 \mathrm{MHz})$ and ${ }^{19} \mathrm{~F}(282 \mathrm{MHz}) \mathrm{NMR}$ spectra were recorded on a Bruker Advance 300 in $\mathrm{CDCl}_{3}$ unless otherwise noted. Chemical shifts $\delta$ are quoted in ppm relatively to the residual solvent peak for $\mathrm{CDCl}_{3}\left(\delta_{\mathrm{H}}=7.26 \mathrm{ppm}\right.$ and $\left.\delta_{\mathrm{C}}=77.16 \mathrm{ppm}\right)$ or $\mathrm{C}_{6} \mathrm{D}_{6}\left(\delta_{\mathrm{H}}=7.16\right.$ ppm and $\delta_{C}=128.06 \mathrm{ppm}$ ) or relatively to the external standard $\mathrm{CFCl}_{3}\left(\delta_{\mathrm{F}}=0.0 \mathrm{ppm}\right)$. Coupling constants $J$ are expressed in hertz and the following abbreviations for multiplicities are used: $\mathrm{s}$ for singlet, $\mathrm{d}$ for doublet, $\mathrm{t}$ for triplet, $\mathrm{q}$ for quartet, $\mathrm{h}$ for heptuplet, $\mathrm{dd}$ for doublet of doublets, qd for quartet of doublets and $\mathrm{m}$ for multiplet.

IR spectra were recorded on a PerkinElmer spectrum 100. HRMS were recorded with a Waters LCP Premier XR spectrometer with a TOF analyzer. 


\section{Synthesis of starting materials}

$\alpha-\mathrm{CF}_{3}$ styrene $\mathbf{1 a}$ was purchased from Fluorochem. $\alpha-\mathrm{CF}_{3}$ styrenes $\mathbf{1 b}, \mathbf{1 c}, \mathbf{1 d}, \mathbf{1 e}, \mathbf{1 f}, \mathbf{1 g}, \mathbf{1} \mathbf{h}, \mathbf{1} \mathbf{i}$, $\mathbf{1 j}, \mathbf{1 k}, \mathbf{1}, \mathbf{1} \mathbf{m}$ and $\mathbf{1 n}$ were synthesized according to the literature. ${ }^{[1]}$

Compound 1u was synthesized according to the literature. ${ }^{[2]}$

$\beta-\mathrm{CF}_{3}$ acrylates $1 \mathbf{u}^{\prime}, \mathbf{1 y}, \mathbf{1 z}$, 1aa, 1ab, 1ac, 1ad, 1ae, 1af, 1ag and 1ai were synthesized according to the literature. ${ }^{[3]}$

Compound 1ak was synthesized according to the literature. ${ }^{[4]}$

\section{2.i. General procedure for the synthesis of $\alpha-\mathrm{CF}_{3}$ styrenes}<smiles>O=C(c1cc[R1]cc1)C(F)(F)F</smiles>

Ao, Ar, As, At

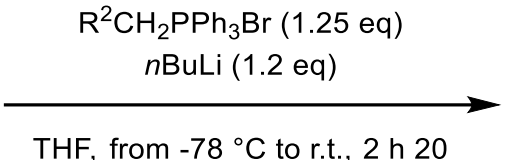

THF, from $-78^{\circ} \mathrm{C}$ to r.t., 2 h 20

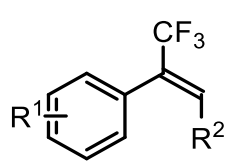

1o, 1r, 1s, 1t

Commercially available ketones $\mathbf{A o}, \mathbf{A r}, \mathbf{A s}$, At and salts $\mathrm{R}^{2} \mathrm{CH}_{2} \mathrm{PPh}_{3} \mathrm{Br}$ were used.

A dry round bottom flask was loaded with $\mathrm{R}^{2} \mathrm{CH}_{2} \mathrm{PPh}_{3} \mathrm{Br}(12.5 \mathrm{mmol})$ and freshly distilled tetrahydrofuran $(30 \mathrm{~mL})$. After cooling at $0{ }^{\circ} \mathrm{C}, n \mathrm{BuLi}(12 \mathrm{mmol}, 2.5 \mathrm{M}$ in hexanes) was added dropwise and the mixture was stirred at $0{ }^{\circ} \mathrm{C}$ for 20 minutes. After cooling to $-78{ }^{\circ} \mathrm{C}$, ketone Ao or Ar or As or At (10 mmol) dissolved in THF $(5 \mathrm{~mL})$ was added dropwise. The mixture was then allowed to warm to room temperature and was stirred for 2 hours. The reaction was quenched with a saturated solution of $\mathrm{NH}_{4} \mathrm{Cl}(30 \mathrm{~mL})$ and the aqueous layer was extracted with diethyl ether $(3 \times 30 \mathrm{~mL})$. The combined organic layers were dried over $\mathrm{Na}_{2} \mathrm{SO}_{4}$, filtered off and concentrated under reduced pressure. The residue was finally purified by column chromatography on silica gel to afford the product 10 or $1 \mathrm{r}$ or $1 \mathrm{~s}$ or $1 \mathrm{t}$.

\section{2.ii. Characterization of $\alpha-\mathrm{CF}_{3}$ styrenes}

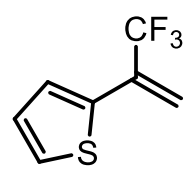

2-(3,3,3-trifluoroprop-1-en-2-yl)thiophene 10: obtained as a pale yellow liquid ( $36 \%$ yield, $1.00 \mathrm{~g})$ after column chromatography on silica gel ( $100 \%$ pentane). Rf: 0.65 (100\% pentane); ${ }^{1} \mathbf{H}$ NMR (300 MHz, $\left.\mathrm{CDCl}_{3}\right): \delta$ 7.30-7.29 (m, 1H), 7.23$7.19(\mathrm{~m}, 1 \mathrm{H}), 7.05-7.02(\mathrm{~m}, 1 \mathrm{H}), 5.84-5.81(\mathrm{~m}, 2 \mathrm{H}) \mathrm{ppm} ;{ }^{13} \mathrm{C} \mathrm{NMR}\left(75 \mathrm{MHz}, \mathrm{CDCl}_{3}\right)$ : $10 \delta 135.8,132.8(q, J=31.3 \mathrm{~Hz}), 128.0,127.0(q, J=1.5 \mathrm{~Hz}), 126.2,122.9(\mathrm{q}, J=$ $272.5 \mathrm{~Hz}), 117.7(\mathrm{q}, J=5.5 \mathrm{~Hz}) \mathrm{ppm} ;{ }^{19} \mathrm{~F}$ NMR $\left(282 \mathrm{MHz}, \mathrm{CDCl}_{3}\right): \delta-66.5 \mathrm{ppm}$; IR (neat): $v$ 3112, 2921, 1628, 1356, 1168, 1122, 1085, 1062, 1027, 925, 698, $642 \mathrm{~cm}^{-1}$; HRMS (El+) m/z: [M] ${ }^{+}$Calcd for $\mathrm{C}_{7} \mathrm{H}_{5} \mathrm{~F}_{3} \mathrm{~S}$ 178.0064; Found 178.0062 . 
<smiles>CCC=C(c1ccccc1)C(F)(F)F</smiles>

$1 \mathrm{r}$

(1,1,1-trifluoropent-2-en-2-yl)benzene 1r: obtained as a mixture of nonseparable diastereomers $(90 \%$ yield, $0.52 \mathrm{~g}$, d.r. $=56: 44)$ after column chromatography on silica gel (100\% pentane). Rf: 0.69 (100\% pentane); ${ }^{1} \mathbf{H}$ NMR (300 MHz, CDCl $)$ : $\delta$ 7.39-7.22 (m, 9H), 6.41 (t, J = 7.5 Hz, 1H, major), 6.01 $(\mathrm{t}, J=7.5 \mathrm{~Hz}, 0.8 \mathrm{H}$, minor), 2.51-2.40 (m, $1.6 \mathrm{H}$, minor), 2.06-1.96 (m, $2 \mathrm{H}$, major), 1.12 (t, $J=7.5 \mathrm{~Hz}, 2.4 \mathrm{H}$, minor), 0.99 (t, $J=7.5 \mathrm{~Hz}, 3 \mathrm{H}$, major) ppm; ${ }^{13} \mathrm{C}$ NMR $\left(75 \mathrm{MHz}, \mathrm{CDCl}_{3}\right): \delta 143.8(q, J=3.0 \mathrm{~Hz}), 138.3(q, J=5.5 \mathrm{~Hz}), 136.9(q, J=1.5 \mathrm{~Hz}), 132.7$, $131.4(q, J=29.3 \mathrm{~Hz}), 130.9$ ( $\mathrm{q}, J=29.3 \mathrm{~Hz}), 129.9,128.6,128.5,128.4-128.3(\mathrm{~m}, 2 \mathrm{C}), 128.1$, $124.3(q, J=274.0 \mathrm{~Hz}), 123.9(q, J=271.0 \mathrm{~Hz}), 22.4(q, J=2.0 \mathrm{~Hz}), 21.9,13.8(q, J=1.3 \mathrm{~Hz})$, 13.3 (q, $J=1.3 \mathrm{~Hz}$ ) ppm; ${ }^{19} \mathrm{~F} \mathrm{NMR}\left(282 \mathrm{MHz}, \mathrm{CDCl}_{3}\right.$ ): $\delta$-57.7 (minor), -66.2 (major) ppm; IR (neat): v 2973, 2880, 1667, 1496, 1461, 1321, 1295, 1202, 1189, 1164, 1111, 895, 763, $699 \mathrm{~cm}^{-}$ 1; HRMS (El+) m/z: [M] ${ }^{+}$Calcd for $\mathrm{C}_{11} \mathrm{H}_{11} \mathrm{~F}_{3}$ 200.0813; Found 200.0813.

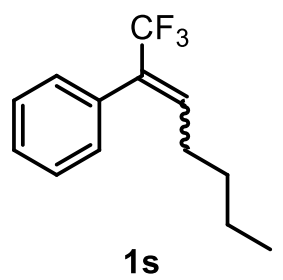

(1,1,1-trifluorohept-2-en-2-yl)benzene 1s: obtained as a mixture of nonseparable diastereomers (99\% yield, $2.02 \mathrm{~g}$, d.r. $=63: 37)$ after column chromatography on silica gel (100\% pentane). Rf: 0.68 (100\% pentane); ${ }^{1} \mathbf{H}$ NMR $\left(300 \mathrm{MHz}_{\mathrm{CDCl}}\right): \delta$ 7.42-7.22 (m, 8H), $6.42(\mathrm{t}, J=7.5 \mathrm{~Hz}, 1 \mathrm{H}$, major), $6.02(\mathrm{t}, J=7.5 \mathrm{~Hz}, 0.6 \mathrm{H}$, minor), 2.48-2.40 (m, $1.2 \mathrm{H}$, minor), 2.04-1.96 (m, $2 \mathrm{H}$, major), $1.54-1.19(\mathrm{~m}, 6.4 \mathrm{H}), 0.94(\mathrm{t}, J=6.0 \mathrm{~Hz}, 1.8 \mathrm{H}$, minor), $0.84(\mathrm{t}, J=$ $7.5 \mathrm{~Hz}, 3 \mathrm{H}$, major) ppm; ${ }^{13} \mathrm{C} \mathrm{NMR}\left(75 \mathrm{MHz}, \mathrm{CDCl}_{3}\right): \delta 142.5(\mathrm{q}, J=3.0 \mathrm{~Hz}), 137.1-136.9(\mathrm{~m}, 2 \mathrm{C})$, $132.8,131.9(q, J=29.5 \mathrm{~Hz}), 131.5(\mathrm{q}, J=29.0 \mathrm{~Hz}), 130.0,128.6,128.5,128.4-128.3(\mathrm{~m}, 2 \mathrm{C})$, $128.1,124.4(q, J=273.8 \mathrm{~Hz}), 124.0(q, J=270.8 \mathrm{~Hz}), 31.7(q, J=1.0 \mathrm{~Hz}), 31.1(q, J=1.0 \mathrm{~Hz})$, $28.7(q, J=2.0 \mathrm{~Hz}), 28.2,22.5,22.3,13.8,13.7$ ppm; ${ }^{19} \mathrm{~F} \mathrm{NMR}\left(282 \mathrm{MHz}, \mathrm{CDCl}_{3}\right): \delta-57.6$ (minor), -66.2 (major) ppm; IR (neat): v 2961, 2931, 2863, 1665, 1496, 1446, 1380, 1302, 1203, 1164, 1112, 766, $699 \mathrm{~cm}^{-1}$; HRMS (El+) m/z: [M] ${ }^{+}$Calcd for $\mathrm{C}_{13} \mathrm{H}_{15} \mathrm{~F}_{3}$ 228.1126; Found 228.1124 .<smiles>FC(F)(F)/C(=C\Cc1ccccc1)c1ccccc1</smiles>

$1 \mathrm{t}$

(4,4,4-trifluorobut-2-ene-1,3-diyl)dibenzene 1t: obtained as a mixture of non-separable diastereomers ( $33 \%$ yield, $0.36 \mathrm{~g}$, d.r. $=50: 50$ ) after column chromatography on silica gel (100\% pentane). Rf: 0.41 (100\% pentane); ${ }^{1} \mathbf{H}$ NMR $\left(300 \mathrm{MHz}, \mathrm{CDCl}_{3}\right): \delta$ 7.46-7.39 (m, 3H), 7.36-7.20 (m, 15H), 7.13-7.10 $(\mathrm{m}, 2 \mathrm{H}), 6.60(\mathrm{t}, J=7.5 \mathrm{~Hz}, 1 \mathrm{H}), 6.17(\mathrm{t}, J=7.5 \mathrm{~Hz}, 1 \mathrm{H}), 3.79(\mathrm{~d}, J=9.0 \mathrm{~Hz}, 2 \mathrm{H})$, $3.35(\mathrm{~d}, J=9.0 \mathrm{~Hz}, 2 \mathrm{H}) \mathrm{ppm} ;{ }^{13} \mathrm{C}$ NMR $\left(75 \mathrm{MHz}, \mathrm{CDCl}_{3}\right): \delta 140.3(\mathrm{q}, J=3.0 \mathrm{~Hz})$, $138.9(q, J=1.0 \mathrm{~Hz}), 138.6(q, J=1.0 \mathrm{~Hz}), 136.4(q, J=1.8 \mathrm{~Hz}), 134.9(q, J=5.5 \mathrm{~Hz}), 132.2-132.1$ ( $\mathrm{m}, 2 \mathrm{C}), 131.8(\mathrm{~d}, J=1.5 \mathrm{~Hz}), 129.9,128.9-128.7(\mathrm{~m}, 5 \mathrm{C}), 128.5-128.3(\mathrm{~m}, 4 \mathrm{C}), 126.8-126.7(\mathrm{~m}$, 2C), $124.1(q, J=274.0 \mathrm{~Hz}), 123.6(q, J=271.3 \mathrm{~Hz}), 35.3(q, J=2.0 \mathrm{~Hz}), 34.6 \mathrm{ppm} ;{ }^{19} \mathrm{~F} \mathrm{NMR}(282$ $\mathrm{MHz}, \mathrm{CDCl}_{3}$ ): $\delta$-57.1, -66.3 ppm; IR (neat): v 3064, 3030, 1664, 1603, 1496, 1378, 1309, 1204, 1152, 1112, 745, 736, $696 \mathrm{~cm}^{-1}$; HRMS (EI+) m/z: [M] ${ }^{+}$Calcd for $\mathrm{C}_{16} \mathrm{H}_{13} \mathrm{~F}_{3}$ 262.0969; Found 262.0968 . 


\section{2.iii. Preparation and characterization of compounds $1 p$ and $1 q$}
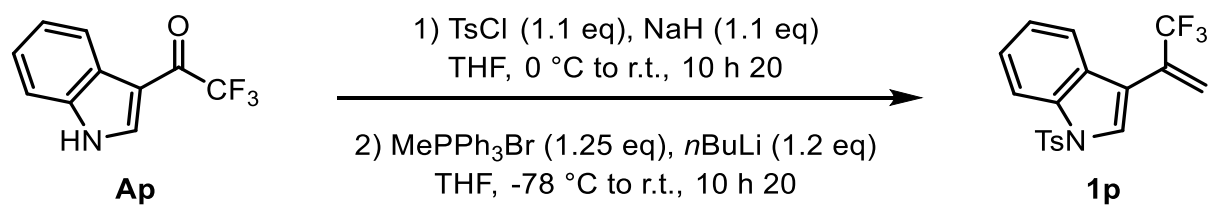

Commercially available ketone Ap was used.

A dry round bottom flask was loaded with $\mathrm{Ap}(10 \mathrm{mmol})$ and freshly distilled tetrahydrofuran $(20 \mathrm{~mL})$. After cooling at $0^{\circ} \mathrm{C} \mathrm{NaH}(11 \mathrm{mmol})$ was added portionwise, the mixture was allowed to warm to room temperature and it was stirred for 20 minutes. $\mathrm{TsCl}(11 \mathrm{mmol})$ was then added and the mixture was stirred at room temperature for 10 hours. The reaction was quenched with brine $(10 \mathrm{~mL})$ and the aqueous layer was extracted with diethyl ether $(3 \times 10$ $\mathrm{mL}$ ). The combined organic layers were dried over $\mathrm{Na}_{2} \mathrm{SO}_{4}$, filtered off and concentrated under reduced pressure. The residue was used in the next step without any further purification.

A dry round bottom flask was loaded with $\mathrm{MePPh}_{3} \mathrm{Br}(12.5 \mathrm{mmol})$ and freshly distilled tetrahydrofuran $(30 \mathrm{~mL})$. After cooling at $0{ }^{\circ} \mathrm{C}, n \mathrm{BuLi}(12 \mathrm{mmol}, 2.5 \mathrm{M}$ in hexanes) was added dropwise and the mixture was stirred at $0{ }^{\circ} \mathrm{C}$ for 20 minutes. After cooling at $-78{ }^{\circ} \mathrm{C}$, ketone resulting from the previous step $(10 \mathrm{mmol})$ dissolved in THF $(5 \mathrm{~mL})$ was added dropwise. The mixture was then allowed to warm to room temperature and was stirred for 2 hours. The reaction was quenched with a saturated solution of $\mathrm{NH}_{4} \mathrm{Cl}(30 \mathrm{~mL})$ and the aqueous layer was extracted with diethyl ether $(3 \times 30 \mathrm{~mL})$. The combined organic layers were dried over $\mathrm{Na}_{2} \mathrm{SO}_{4}$, filtered off and concentrated under reduced pressure. The residue was finally purified by column chromatography on silica gel to afford the product $\mathbf{1 p}$.

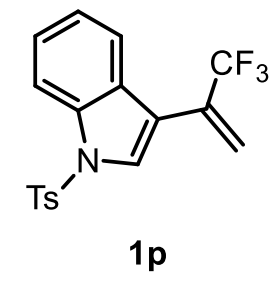

1-tosyl-3-(3,3,3-trifluoroprop-1-en-2-yl)-1H-indole 1p: obtained as a white amorphous solid (61\% yield, $1.20 \mathrm{~g}$ ) after column chromatography on silica gel (pentane:Et ${ }_{2} \mathrm{O}$, from 100:0 to 80:20). Rf: 0.43 (PE:Et $\left.{ }_{2} \mathrm{O}, 80: 20\right) ;{ }^{1} \mathbf{H} \mathbf{~ N M R}$ $\left(300 \mathrm{MHz}, \mathrm{CDCl}_{3}\right): \delta 8.02(\mathrm{~d}, J=9.0 \mathrm{~Hz}, 1 \mathrm{H}), 7.80-7.74(\mathrm{~m}, 3 \mathrm{H}), 7.66(\mathrm{~d}, J=9.0$ $\mathrm{Hz}, 1 \mathrm{H}), 7.39-7.23(\mathrm{~m}, 4 \mathrm{H}), 6.12(\mathrm{~s}, 1 \mathrm{H}), 5.95(\mathrm{~s}, 1 \mathrm{H}), 2.35(\mathrm{~s}, 3 \mathrm{H}) \mathrm{ppm} ;{ }^{13} \mathrm{C}$ NMR $\left(75 \mathrm{MHz}, \mathrm{CDCl}_{3}\right): \delta 145.5,135.0(\mathrm{q}, J=4.5 \mathrm{~Hz}), 131.2,130.8,130.2$, $128.9,127.1,125.4-125.3(\mathrm{~m}, 2 \mathrm{C}), 124.0,123.0$ (q, $J=272.3 \mathrm{~Hz}), 120.8(\mathrm{q}, J=5.5 \mathrm{~Hz}$ ), 120.3, 114.8, 113.9, 21.7 ppm; $\left.{ }^{19} \mathrm{~F} \mathrm{NMR} \mathrm{(282} \mathrm{MHz,} \mathrm{CDCl}\right)$ ): $\delta$-67.1 ppm; IR (neat): v 3134, 3082, 1596, 1370, 1176, 1125, 1088, 963, 742, 671, 586, 574, $536 \mathrm{~cm}^{-1}$; HRMS (ES+) m/z: [M + NH $]^{+}$Calcd for $\mathrm{C}_{18} \mathrm{H}_{18} \mathrm{~F}_{3} \mathrm{~N}_{2} \mathrm{O}_{2} \mathrm{~S} 383.1041$; Found 383.1058. 


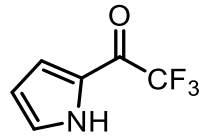

Aq

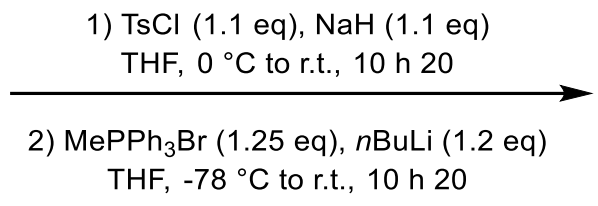

) $\mathrm{TsCl}(1.1 \mathrm{eq}), \mathrm{NaH}(1.1 \mathrm{eq})$

${ }^{\circ} \mathrm{C}$ to r.t, $10 \mathrm{~h} 20$

THF, $-78^{\circ} \mathrm{C}$ to r.t., $10 \mathrm{~h} 20$

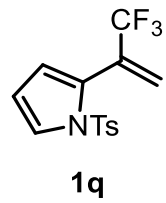

$1 q$

Commercially available ketone Aq was used.

The same procedure as the one used for the synthesis of $1 p$ was used.<smiles></smiles>

$1 q$

1-tosyl-2-(3,3,3-trifluoroprop-1-en-2-yl)-1H-pyrrole 1q: obtained as an orange liquid $(66 \%$ yield, $1.30 \mathrm{~g})$ after column chromatography on silica gel (pentane:Et ${ }_{2} \mathrm{O}$, from 100:0 to 80:20). Rf: 0.42 (PE:Et $\left.{ }_{2} \mathrm{O}, 80: 20\right) ;{ }^{1} \mathrm{H} \mathrm{NMR}(300 \mathrm{MHz}$, $\left.\mathrm{CDCl}_{3}\right): \delta$ 7.58-7.55 (m, 2H), 7.44-7.42 (m, $\left.1 \mathrm{H}\right), 7.24-7.21(\mathrm{~m}, 2 \mathrm{H}), 6.31-6.24(\mathrm{~m}$, $\left.\mathrm{CDCl}_{3}\right): \delta 145.4,135.7,129.9,129.0(\mathrm{q}, J=32.3 \mathrm{~Hz}), 127.3-127.2(\mathrm{~m}, 2 \mathrm{C}), 126.1,125.5,122.3$ $(\mathrm{q}, J=272.0 \mathrm{~Hz}), 117.5,112.0,21.6 \mathrm{ppm} ;{ }^{19} \mathrm{~F}$ NMR $\left(282 \mathrm{MHz}, \mathrm{CDCl}_{3}\right): \delta-67.0 \mathrm{ppm}$; IR (neat): v 3152, 2987, 2874, 1597, 1371, 1173, 1144, 1076, 666, 588, 580, $541 \mathrm{~cm}^{-1}$; HRMS (AP+) m/z: $[\mathrm{M}+\mathrm{H}]^{+}$Calcd for $\mathrm{C}_{14} \mathrm{H}_{13} \mathrm{~F}_{3} \mathrm{NO}_{2} \mathrm{~S} 316.0619$; Found 316.0628 .

\section{2.iv. Preparation and characterization of compounds $1 v, 1 w$ and $1 x$}<smiles>OC/C=C(\c1ccccc1)C(F)(F)F</smiles>

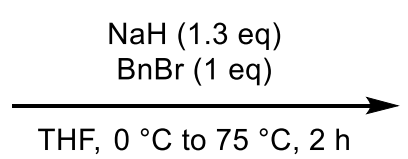

$\mathrm{THF}, 0^{\circ} \mathrm{C}$ to $75^{\circ} \mathrm{C}, 2 \mathrm{~h}$<smiles>FC(F)(Br)CCOCC=C(c1ccccc1)C(F)(F)F</smiles>

1v

A dry round bottom flask was loaded with $1 \mathbf{u}(1.5 \mathrm{mmol})$ and freshly distilled tetrahydrofuran $(5 \mathrm{~mL})$. It was cooled at $0{ }^{\circ} \mathrm{C}, \mathrm{NaH}(1.9 \mathrm{mmol})$ was added to the mixture and it was stirred at 0 ${ }^{\circ} \mathrm{C}$ for 1 hour. $\mathrm{BnBr}(1.5 \mathrm{mmol})$ was then added and the mixture was heated to $75^{\circ} \mathrm{C}$ for 1 hour. After cooling to room temperature, the reaction was quenched with a saturated solution of $\mathrm{NH}_{4} \mathrm{Cl}(10 \mathrm{~mL})$ and the aqueous layer was extracted with diethyl ether $(3 \times 10 \mathrm{~mL})$. The combined organic layers were dried over $\mathrm{Na}_{2} \mathrm{SO}_{4}$, filtered off and concentrated under reduced pressure. The residue was finally purified by column chromatography on silica gel to afford the product $\mathbf{1 v}$.<smiles>FC(F)(F)C(=CCOc1ccccc1)c1ccccc1</smiles>

1v
(E)-(4-(benzyloxy)-1,1,1-trifluorobut-2-en-2-yl)benzene 1v: obtained as a yellow oil ( $18 \%$ yield, $79 \mathrm{mg}$ ) after column chromatography on silica gel (pentane:Et ${ }_{2} \mathrm{O}$, from 100:0 to 95:5). Rf: 0.68 (PE:Et $\left.{ }_{2} \mathrm{O}, 80: 20\right) ;{ }^{1} \mathbf{H}$ NMR (300 $\left.\mathrm{MHz}, \mathrm{CDCl}_{3}\right): \delta$ 7.40-7.21 (m, 10H), 6.62-6.58 (m, 1H), $4.44(\mathrm{~s}, 2 \mathrm{H}), 4.02-3.99$ (m, 2H) ppm; $\left.{ }^{13} \mathrm{C} \mathrm{NMR} \mathrm{(75} \mathrm{MHz,} \mathrm{CDCl}_{3}\right): \delta 137.7,133.8-132.6(\mathrm{~m}, 2 \mathrm{C}), 131.6$, $129.4,129.0,128.6-128.5(\mathrm{~m}, 2 \mathrm{C}), 128.0,127.9,123.2$ (q, $J=271.5 \mathrm{~Hz}$ ), 
72.9, 66.4 ppm; ${ }^{19} \mathrm{~F}$ NMR (282 MHz, $\left.\mathrm{CDCl}_{3}\right): \delta$-66.7 ppm; HRMS (El+) m/z: [M] ${ }^{+}$Calcd for $\mathrm{C}_{17} \mathrm{H}_{15} \mathrm{~F}_{3} \mathrm{O}$ 292.1075; Found 292.1070.<smiles>OC/C=C(\c1ccccc1)C(F)(F)F</smiles>

$1 \mathrm{u}$

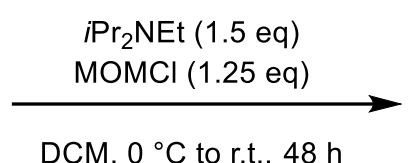

DCM, $0{ }^{\circ} \mathrm{C}$ to r.t., $48 \mathrm{~h}$<smiles>COC/C=C(\c1ccccc1)C(F)(F)F</smiles>

$1 w$

A dry round bottom flask was loaded with $1 \mathrm{u}(1.5 \mathrm{mmol})$ and freshly distilled dichloromethane $(10 \mathrm{~mL})$. It was cooled at $0{ }^{\circ} \mathrm{C}, i \operatorname{Pr}_{2} \mathrm{NEt}(2.2 \mathrm{mmol})$ was added to the mixture, followed by $\mathrm{MOMCl}(1.9 \mathrm{eq})$. It was then allowed to warm to room temperature and the mixture was stirred for 48 hours. After quenching with brine $(10 \mathrm{~mL})$, the aqueous layer was extracted with dichloromethane $(3 \times 10 \mathrm{~mL})$, the combined organic layers were dried over $\mathrm{Na}_{2} \mathrm{SO}_{4}$, filtered off and concentrated under reduced pressure. The residue was finally purified by column chromatography on silica gel to afford the product $\mathbf{1} \mathbf{w}$.<smiles>COC/C=C(\c1ccccc1)C(F)(F)F</smiles>

$1 \mathrm{w}$

(E)-(1,1,1-trifluoro-4-(methoxymethoxy)but-2-en-2-yl)benzene 1w: obtained as a pale yellow liquid ( $36 \%$ yield, $1.00 \mathrm{~g}$ ) after column chromatography on silica gel (PE:Et ${ }_{2} \mathrm{O}$, from 100:0 to 90:10). Rf: 0.67 (PE: $\left.\mathrm{Et}_{2} \mathrm{O}, 80: 20\right) ;{ }^{1} \mathrm{H}$ NMR (300 MHz, $\left.\mathrm{CDCl}_{3}\right): \delta$ 7.43-7.38 (m, 3H), 7.28$7.22(\mathrm{~m}, 2 \mathrm{H}), 6.58-6.54(\mathrm{~m}, 1 \mathrm{H}), 4.58(\mathrm{~s}, 2 \mathrm{H}), 4.05-4.02(\mathrm{~m}, 2 \mathrm{H}), 3.32(\mathrm{~s}$, 3H) ppm; ${ }^{13} \mathrm{C}$ NMR (75 MHz, CDCl 3$): \delta 133.2$ (q, $\left.J=30.0 \mathrm{~Hz}\right), 132.9$ (q, $J=$ $5.5 \mathrm{~Hz}$ ), 131.5, 129.4, 129.0, 128.6, 123.2 (q, $J=271.5 \mathrm{~Hz}$ ), 96.3, 63.9, 55.3 ppm; ${ }^{19} \mathrm{~F}$ NMR (282 $\mathrm{MHz}, \mathrm{CDCl}_{3}$ ): $\delta$-66.7 ppm; IR (neat): v 2936, 2885, 1497, 1446, 1351, 1291, 1171, 1151, 1117, 1043, 1030, 994, 970, 920, $702 \mathrm{~cm}^{-1}$; HRMS (El+) m/z: [M] Calcd for $\mathrm{C}_{12} \mathrm{H}_{13} \mathrm{~F}_{3} \mathrm{O}_{2}$ 246.0868; Found 246.0866 .<smiles>OC/C=C(/c1ccccc1)C(F)(F)F</smiles>

1u

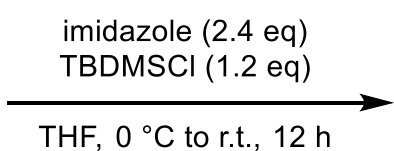

THF, $0{ }^{\circ} \mathrm{C}$ to r.t., $12 \mathrm{~h}$<smiles>CS(C)(C)O[O+]C/C=C(/c1ccccc1)C(F)(F)F</smiles>

$1 \mathrm{x}$

A dry round bottom flask was loaded with $1 \mathbf{u}(1.5 \mathrm{mmol})$ and freshly distilled tetrahydrofuran (6 mL). It was cooled at $0{ }^{\circ} \mathrm{C}$ and imidazole $(3.6 \mathrm{mmol})$ was added. TBDMSCl $(1.8 \mathrm{eq})$ in tetrahydrofuran $(4 \mathrm{~mL})$ was then slowly added to the mixture and it was stirred at $0{ }^{\circ} \mathrm{C}$ for 2 hours. It was then allowed to warm to room temperature and was stirred for 10 additional hours. After quenching with a saturated solution of $\mathrm{NH}_{4} \mathrm{Cl}(10 \mathrm{~mL})$, the aqueous layer was extracted with diethyl ether $(3 \times 10 \mathrm{~mL})$, the combined organic layers were dried over $\mathrm{Na}_{2} \mathrm{SO}_{4}$, filtered off and concentrated under reduced pressure. The residue was finally purified by column chromatography on silica gel to afford the product $\mathbf{1 x}$. 
$\underbrace{c F_{3}}_{\text {OTBDMS }}$

$1 x$

(E)-tert-butyldimethyl((4,4,4-trifluoro-3-phenylbut-2-en-1yl)oxy)silane 1x: obtained as a pale yellow liquid (99\% yield, $0.47 \mathrm{~g}$ ) after column chromatography on silica gel (PE:Et ${ }_{2} \mathrm{O}$, from 100:0 to 90:10). Rf: 0.42 (PE:Et $\left.{ }_{2} \mathrm{O}, 80: 20\right) ;{ }^{1} \mathbf{H}$ NMR (300 MHz, $\mathrm{CDCl}_{3}$ ): $\delta 7.40-$ $7.38(\mathrm{~m}, 3 \mathrm{H}), 7.23-7.19(\mathrm{~m}, 2 \mathrm{H}), 6.52-6.48(\mathrm{~m}, 1 \mathrm{H}), 4.13-4.11(\mathrm{~m}, 2 \mathrm{H})$, 0.85 (s, 9H), 0.01 (s, 6H) ppm; ${ }^{13} \mathrm{C} \mathrm{NMR} \mathrm{(75} \mathrm{MHz,} \mathrm{CDCl} 3$ ): $\delta 136.2$ (q, J $=5.3 \mathrm{~Hz}$ ), 131.8, 131.3 (q, $J=29.8 \mathrm{~Hz}), 129.5,129.0,128.6,123.4$ (q, $J=271.5 \mathrm{~Hz}), 60.0,25.9$, 18.4, -5.2 ppm; ${ }^{19} \mathrm{~F}$ NMR (282 MHz, CDCl $): \delta$-66.5 ppm; IR (neat): v 2956, 2932, 2859, 1496, $1473,1351,1295,1253,1171,1121,1074,909,833,814,775,739,700,672 \mathrm{~cm}^{-1}$; HRMS (Cl+) $\mathrm{m} / \mathrm{z}:[\mathrm{M}+\mathrm{H}]^{+}$Calcd for $\mathrm{C}_{16} \mathrm{H}_{24} \mathrm{~F}_{3} \mathrm{OSi}$ 317.1549; Found 317.1542.

\section{2.v. General procedure for the synthesis of $\beta-\mathrm{CF}_{3}$ acrylates}<smiles>O=C(c1cc[R1]cc1)C(F)(F)F</smiles>

Aah, Aaj

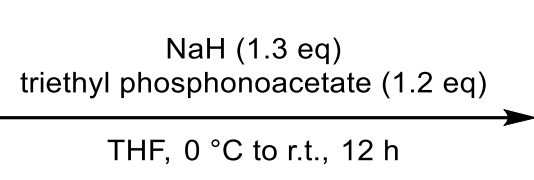

THF, $0{ }^{\circ} \mathrm{C}$ to r.t., 12

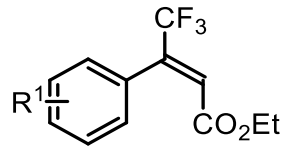

1ah, 1aj

Commercially available ketones Aah and Aaj were used.

A dry round bottom flask was loaded with $\mathrm{NaH}(13 \mathrm{mmol})$ and freshly distilled tetrahydrofuran $(40 \mathrm{~mL})$. It was cooled at $0{ }^{\circ} \mathrm{C}$ and triethyl phosphonoacetate $(12 \mathrm{mmol})$ was slowly added to the mixture, which was then allowed to warm to room temperature and stirred for 2 hours. Ketone Aah or Aaj (10 mmol) was then added dropwise to the mixture and it was stirred at room temperature for 10 hours. After quenching at $0{ }^{\circ} \mathrm{C}$ with a saturated solution of $\mathrm{NH}_{4} \mathrm{Cl}(40$ $\mathrm{mL})$, the aqueous layer was extracted with diethyl ether $(3 \times 30 \mathrm{~mL})$ and the combined organic layers were washed with brine $(100 \mathrm{~mL})$, dried over $\mathrm{Na}_{2} \mathrm{SO}_{4}$, filtered off and concentrated under reduced pressure. The residue was finally purified by column chromatography on silica gel to afford the product 1 ah or $\mathbf{1}$ aj.

\section{2.vi. Characterization of $\beta-\mathrm{CF}_{3}$ acrylates}<smiles>CCOC(=O)C=C(OCC)c1cccc(Cl)c1</smiles>

$1 \mathrm{ah}$

Ethyl (E)-3-(3-chlorophenyl)-4,4,4-trifluorobut-2-enoate 1ah: obtained as a pale yellow liquid ( $41 \%$ yield, $1.66 \mathrm{~g}$ ) after column chromatography on silica gel (PE:Et ${ }_{2} \mathrm{O}$, from 100:0 to 98:2). Rf: 0.64 (PE:Et $\left.{ }_{2} \mathrm{O}, 80: 20\right) ;{ }^{1} \mathbf{H}$ NMR $\left(300 \mathrm{MHz}, \mathrm{CDCl}_{3}\right): \delta$ 7.43-7.28 $(\mathrm{m}, 3 \mathrm{H}), 7.19-7.16(\mathrm{~m}, 1 \mathrm{H}), 6.63-$ $6.62(\mathrm{~m}, 1 \mathrm{H}), 4.07(\mathrm{q}, J=7.2 \mathrm{~Hz}, 2 \mathrm{H}), 1.09(\mathrm{t}, J=7.5 \mathrm{~Hz}, 3 \mathrm{H}) \mathrm{ppm} ;{ }^{13} \mathrm{C}$ NMR $\left(75 \mathrm{MHz}, \mathrm{CDCl}_{3}\right): \delta 163.7,140.9(\mathrm{q}, J=31.3 \mathrm{~Hz}), 134.2,132.8$, 129.6-129.5 (m, 2C), 128.9, 127.0, 125.5 (q, $J=5.5 \mathrm{~Hz}$ ), 122.4 (q, $J=272.8 \mathrm{~Hz}$ ), 61.3, $13.7 \mathrm{ppm}$; ${ }^{19} \mathrm{~F}$ NMR $\left(282 \mathrm{MHz}, \mathrm{CDCl}_{3}\right)$ : $\delta$-68.0 ppm; IR (neat): v 2987, 1733, 1282, 1255, 1203, 1174, 1130, 1028, 786, $699 \mathrm{~cm}^{-1}$; HRMS (El+) m/z: [M] Calcd for $\mathrm{C}_{12} \mathrm{H}_{10} \mathrm{ClF}_{3} \mathrm{O}_{2} 278.0321$; Found 278.0320. 
<smiles>CCOC(=O)C=C(OCC)c1cccs1</smiles>

1aj

Ethyl (Z)-4,4,4-trifluoro-3-(thiophen-2-yl)but-2-enoate 1aj: obtained as a yellow liquid ( $42 \%$ yield, $1.16 \mathrm{~g}$ ) after column chromatography on silica gel (pentane:acetone, from 100:0 to 98:2). Rf: 0.61 (PE:Et $2 \mathrm{O}, 80: 20) ;{ }^{1} \mathbf{H} \mathbf{~ N M R}$ (300 MHz, $\left.\mathrm{CDCl}_{3}\right): \delta$ 7.49-7.47 (m, 1H), 7.25-7.24 (m, 1H), 7.09-7.06 (m, 1H), $6.60(\mathrm{~m}, 1 \mathrm{H}), 4.17(\mathrm{q}, J=7.0 \mathrm{~Hz}, 2 \mathrm{H}), 1.19(\mathrm{t}, J=7.5 \mathrm{~Hz}, 3 \mathrm{H}) \mathrm{ppm} ;{ }^{13} \mathrm{C}$ NMR (75 MHz, $\mathrm{CDCl}_{3}$ ): $\delta$ 164.4, 134.3 (q, $J=31.8 \mathrm{~Hz}$ ), 130.5, 130.1, 128.8, 127.1, 124.6 (q, $J=5.5$ $\mathrm{Hz}$ ), 122.3 (q, $J=273.8 \mathrm{~Hz}$ ), 61.5, 13.9 ppm; ${ }^{19} \mathrm{~F} \mathrm{NMR} \mathrm{(282} \mathrm{MHz,} \mathrm{CDCl} 3$ ): $\delta-67.8$ ppm; IR (neat): v 2986, 1728, 1280, 1257, 1231, 1177, 1129, 1025, $704 \mathrm{~cm}^{-1}$; HRMS (El+) m/z: [M] Calcd for $\mathrm{C}_{10} \mathrm{H}_{9} \mathrm{~F}_{3} \mathrm{O}_{2} \mathrm{~S} 250.0275$; Found 250.0274. 


\section{Optimization \& mechanistic studies}

\section{3.i. Optimization}<smiles>C=C(c1ccccc1)C(F)(F)F</smiles>

$1 \mathrm{a}$

$0.23 \mathrm{mmol}$

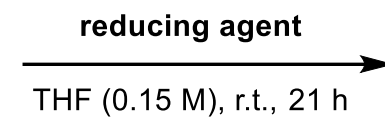

THF (0.15 M), r.t., $21 \mathrm{~h}$

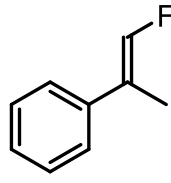

$2 a$

\begin{tabular}{cccc}
\hline Entry & Reducing agent & Yield $^{\mathbf{a}} \mathbf{( \% )}^{\mathbf{b}}$ & d.r. $^{\mathbf{c}}$ \\
\hline $\mathbf{1}$ & $\mathrm{LiAlH}_{4}(1 \mathrm{eq})$ & $78(56)$ & $95: 5$ \\
\hline $\mathbf{2}$ & $\mathrm{DIBAL}^{(4}$ eq) & 0 & - \\
\hline $\mathbf{3}$ & $\operatorname{RedAl}^{\circledR}(2$ eq) & 62 & $85: 15$ \\
\hline $\mathbf{4}$ & $\mathrm{NaBH}_{4}(1 \mathrm{eq})$ & $\mathrm{NR}$ & - \\
\hline $\mathbf{5}$ & $\mathrm{LiBH}_{4}(1$ eq) & $\mathrm{NR}$ & -
\end{tabular}

a Yield determined by ${ }^{19} \mathrm{~F}$ NMR using 4-nitrofluorobenzene as an internal standard. ${ }^{\mathrm{b}}$ Isolated yield. ${ }^{\mathrm{c}}$ Determined by ${ }^{19} \mathrm{~F}$ NMR on the crude reaction mixture.

\section{Entry 2:}

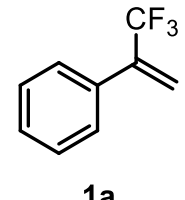

$(0.23 \mathrm{mmol})$

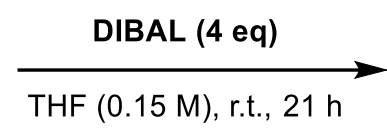

THF (0.15 M), r.t., $21 \mathrm{~h}$

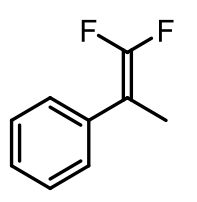

$2 a^{\prime}$

$74 \%\left({ }^{19} \mathrm{~F}\right.$ NMR yield $)$ 


\section{3.ii. Mechanistic studies}

$\underline{\text { Substrate geometry effect: }}$<smiles>CCOC(=O)/C=C(\c1ccc(F)cc1)C(F)(F)F</smiles>

(E)-1 af

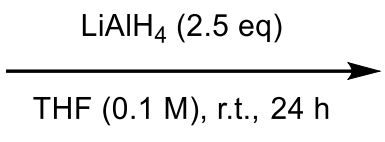

$\mathrm{LiAlH}_{4}(2.5 \mathrm{eq})$

$\operatorname{THF}(0.1 \mathrm{M})$, r.t., $24 \mathrm{~h}$<smiles>OCC/C(=C\F)c1ccc(F)cc1</smiles>

(E)-2af

$71 \%$ d.r. $=74: 26$

\section{$\underline{\text { Reaction's intermediates: }}$}<smiles>C=C(c1ccc(OC)cc1)C(F)(F)F</smiles>

3

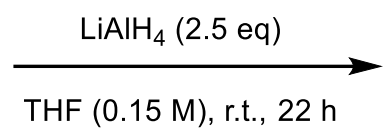

THF (0.15 M), r.t., $22 \mathrm{~h}$

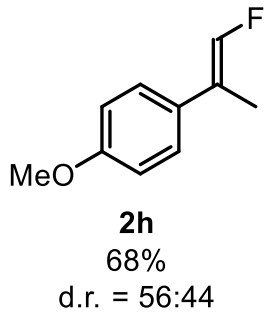<smiles>C=C(c1ccc(OC)cc1)C(F)(F)F</smiles>

$1 \mathrm{~h}$<smiles>COc1ccc(C(C)=C(F)F)cc1</smiles>

$98 \%$

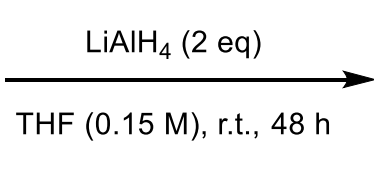

$\mathrm{MeO}$

$99 \%{ }^{a}$

d.r. $=95: 5$

${ }^{\text {a }}$ Yield determined by ${ }^{19} \mathrm{~F}$ NMR using 4-nitrofluorobenzene as an internal standard. 
<smiles>COc1ccc(C(C)=C(F)F)cc1</smiles>

4

1-(1,1-difluoroprop-1-en-2-yl)-4-methoxybenzene $\mathbf{2} \mathbf{h}^{\prime}$ : obtained as a light yellow liquid ( $98 \%$ yield, $72 \mathrm{mg}$ ) after column chromatography on silica gel (pentane:Et ${ }_{2} \mathrm{O}$, from 100:0 to 90:10). ${ }^{1} \mathbf{H}$ NMR (300 MHz, $\mathrm{CDCl}_{3}$ ): $\delta$ 7.31$7.28(\mathrm{~m}, 2 \mathrm{H}), 6.92-6.86(\mathrm{~m}, 2 \mathrm{H}), 3.81(\mathrm{~s}, 3 \mathrm{H}), 1.95-1.93(\mathrm{~m}, 3 \mathrm{H}) \mathrm{ppm} ;{ }^{13} \mathrm{C}$ NMR $\left(75 \mathrm{MHz} \mathrm{CDCl}_{3}\right): \delta$ 158.6, $153.4(\mathrm{dd}, J=286.9 \& 283.9 \mathrm{~Hz}), 128.7$ (dd, $J=4.5$ \& $3.8 \mathrm{~Hz}), 127.3-127.1(\mathrm{~m}, 2 \mathrm{C}), 113.9,55.4,13.5-13.4(\mathrm{~m}) \mathrm{ppm} ;{ }^{19} \mathrm{~F}$

NMR $\left(282 \mathrm{MHz}, \mathrm{CDCl}_{3}\right): \delta-92.2,-92.5$ ppm; IR (neat): v 3004, 2964, 2937, 2838, 1727, 1610, 1514, 1292, 1235, 1181, 1134, 1104, 1032, 899, 829, $559 \mathrm{~cm}^{-1}$; HRMS (El+) m/z: [M] Calcd for $\mathrm{C}_{10} \mathrm{H}_{10} \mathrm{~F}_{2} \mathrm{O}$ 184.0700; Found 184.0704.

\section{Deuteration experiments:}<smiles>C=C(c1ccc2ccccc2c1)C(F)(F)F</smiles>

$1 \mathrm{~g}$

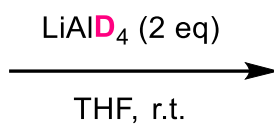

THF, r.t.<smiles>[2H]C(F)=C(c1ccc2ccccc2c1)C([2H])[2H]</smiles>

[D]-2g

$99 \%^{\mathrm{a}}$

d.r. $=93: 7$

${ }^{a}$ Yield determined by ${ }^{19} \mathrm{~F}$ NMR using 4-nitrofluorobenzene as an internal standard.
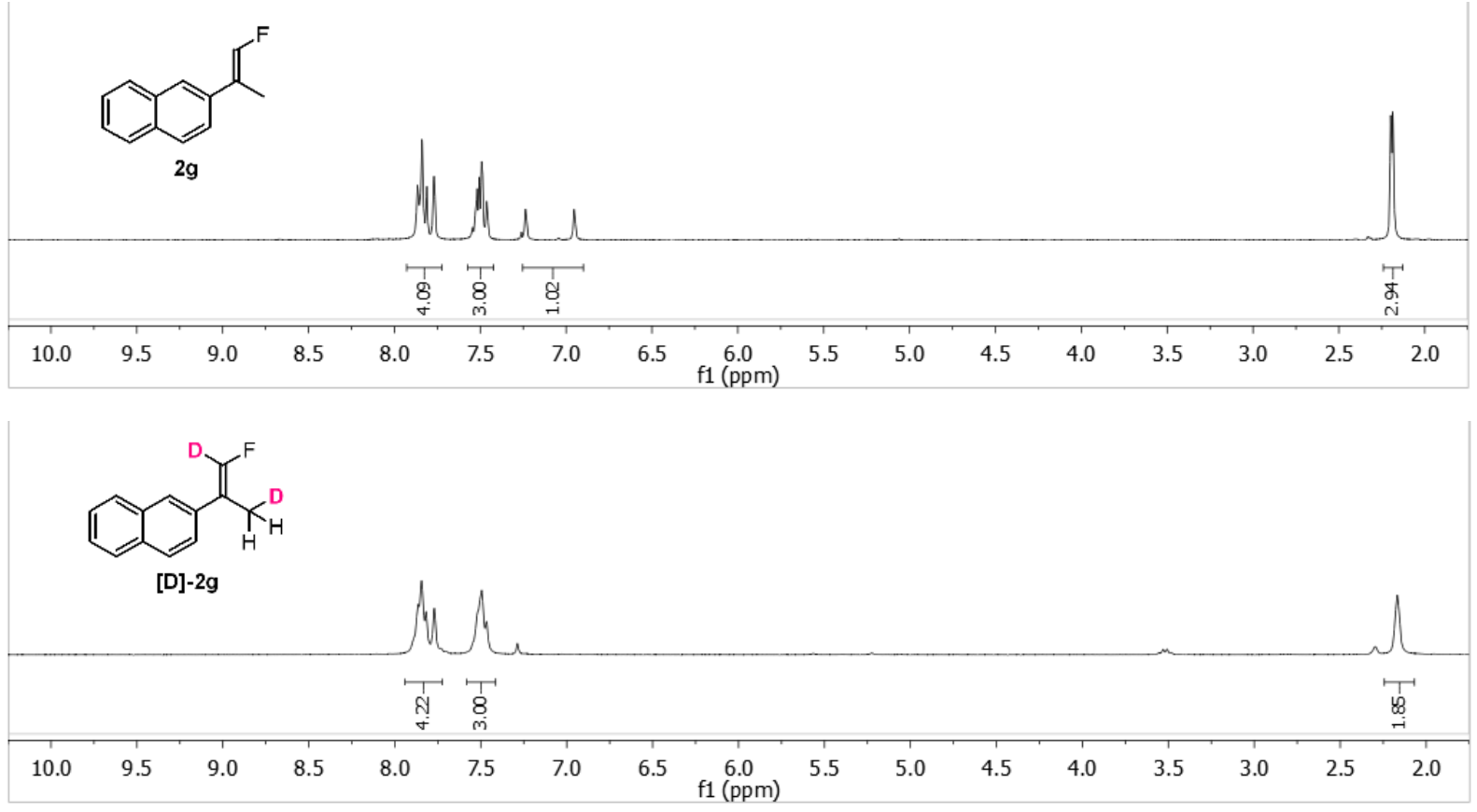


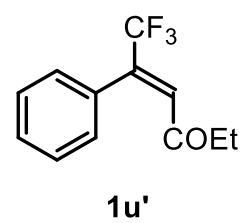

$1 u^{\prime}$

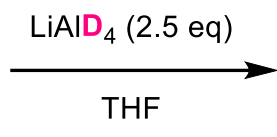

THF<smiles>[2H]C(F)=C(c1ccccc1)C([2H])C([2H])([2H])O</smiles>

[D]-2u

$99 \%^{a}$

d.r. $=74: 26$

a Yield determined by ${ }^{19} \mathrm{~F}$ NMR using 4-nitrofluorobenzene as an internal standard.

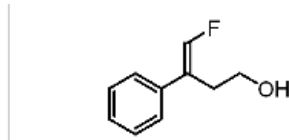

$2 u$
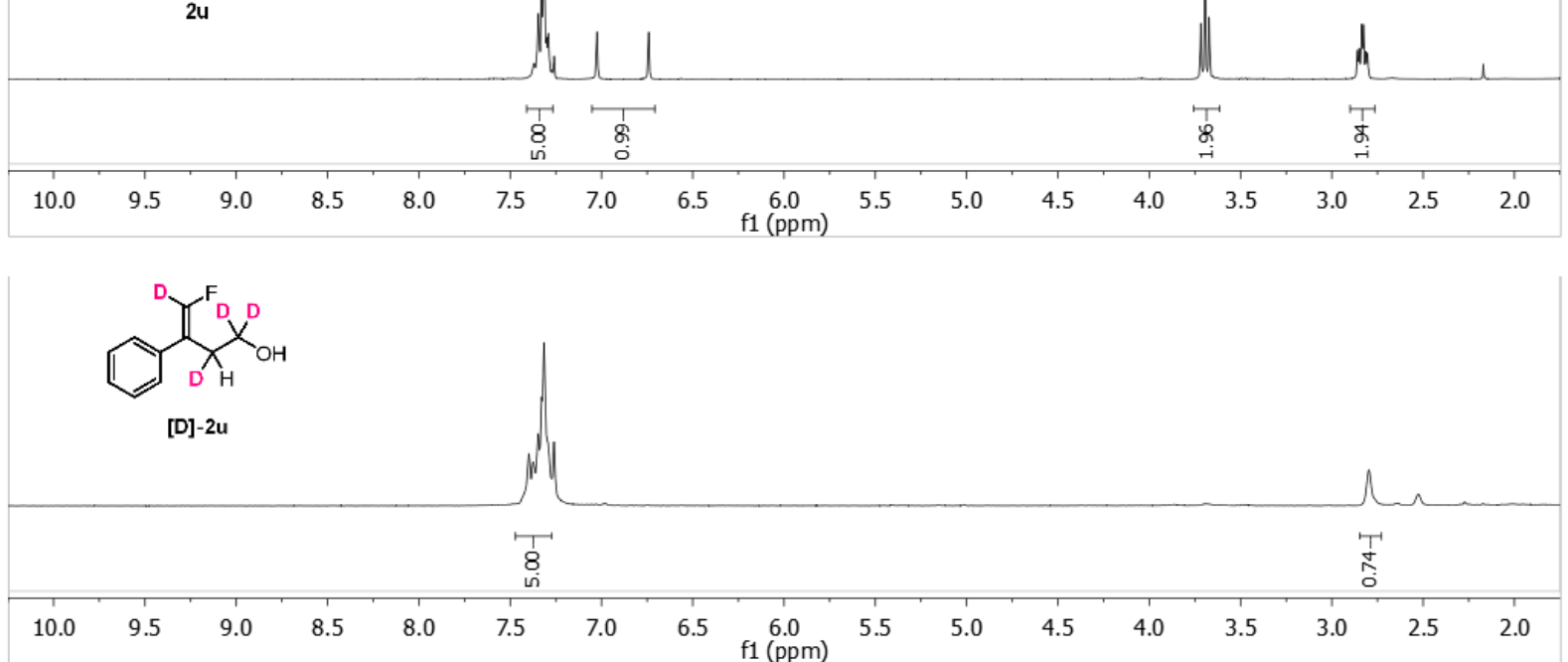
4. Determination of the stereochemistry of the monofluoroalkenes

$\underline{\text { HOESY NMR spectrum of } 2 \mathrm{c}}$

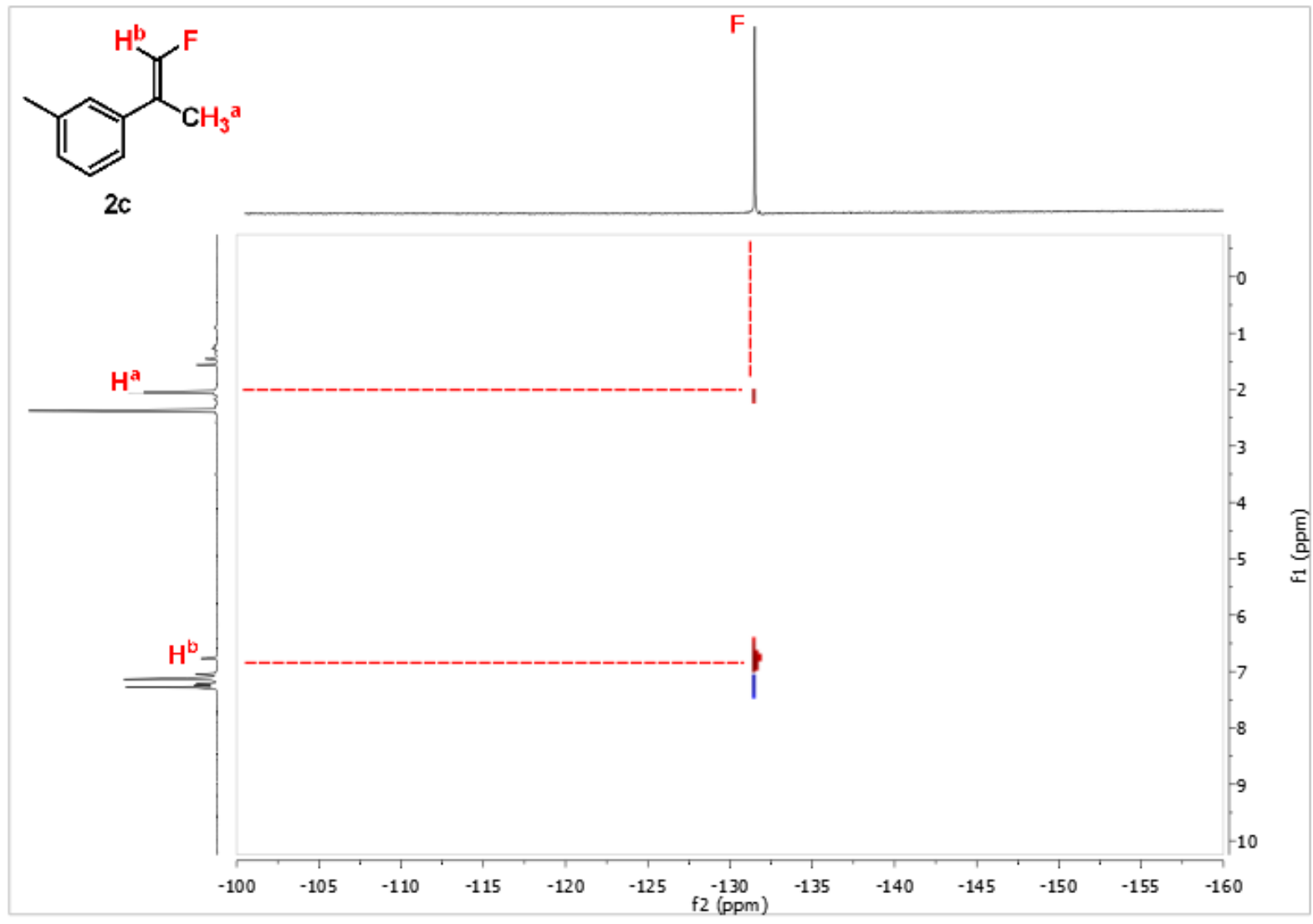


HOESY NMR spectra of $2 \mathbf{u}:$
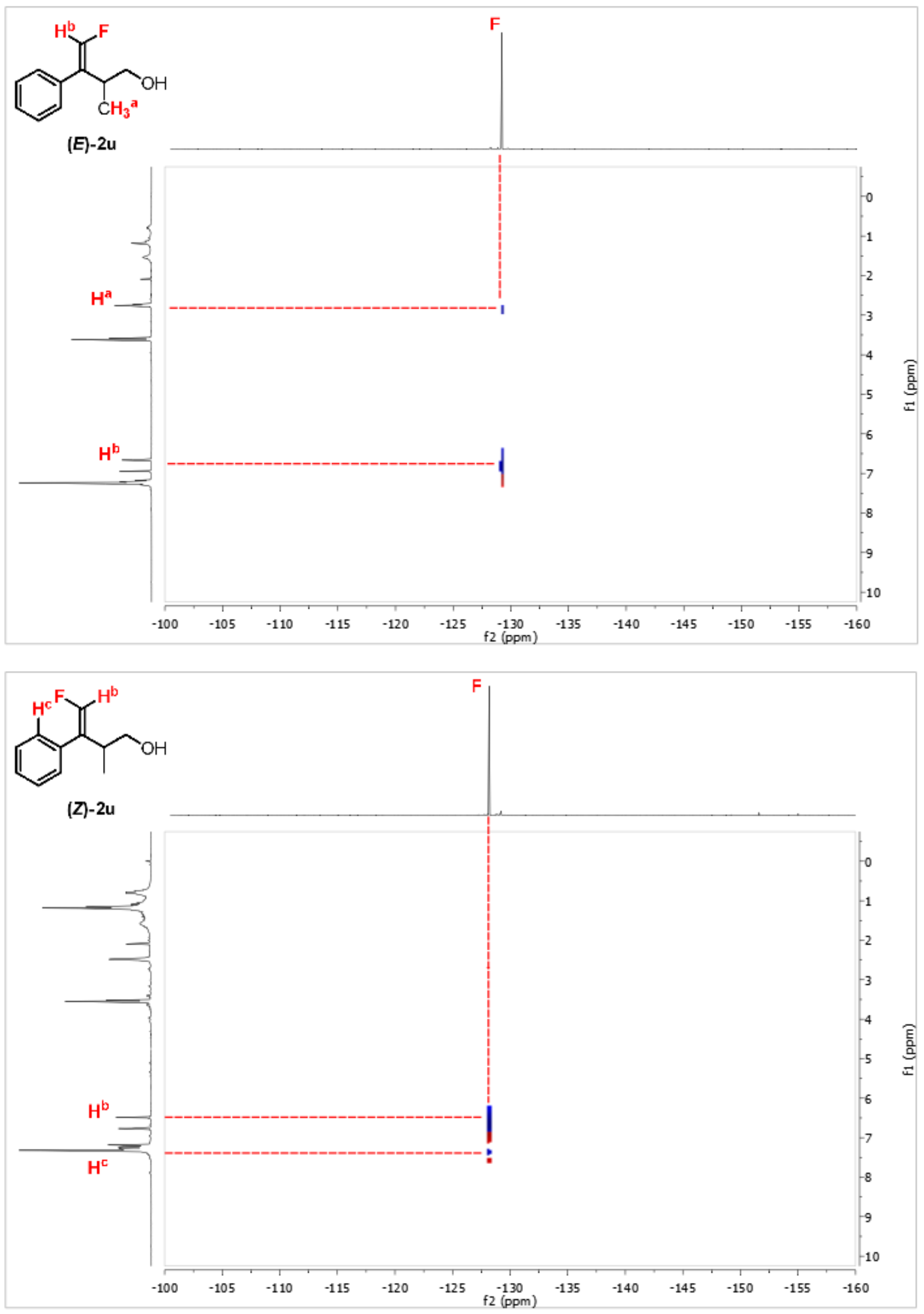
5. Reduction of $\beta-\mathrm{CF}_{3}$ nitrostyrene and acrylonitrile<smiles>O=[N+]([O-])C=C(c1ccccc1)C(F)(F)F</smiles>

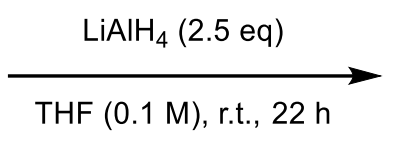<smiles>O=[N+]([O-])CC(c1ccccc1)C(F)(F)F</smiles>

$100 \%$ conversion<smiles>N#C/C=C(\c1ccccc1)C(F)(F)F</smiles>

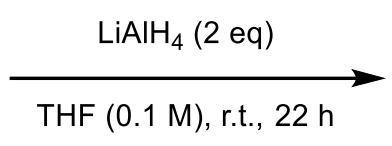<smiles>NCCC(c1ccccc1)C(F)(F)C(F)(F)F</smiles> 


\section{Synthesis of monofluoroalkenes}

\section{6.i. General procedures}

Caution: several compounds proved to be highly volatile and particular attention should be paid.

Procedure for the reduction of $\alpha-\mathrm{CF}_{3}$ styrenes 1a-1r:

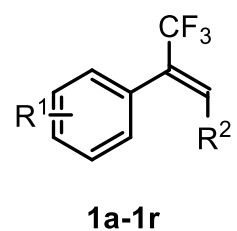

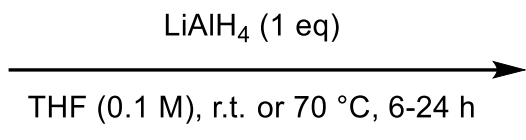

$\operatorname{THF}(0.1 \mathrm{M})$, r.t. or $70^{\circ} \mathrm{C}, 6-24 \mathrm{~h}$

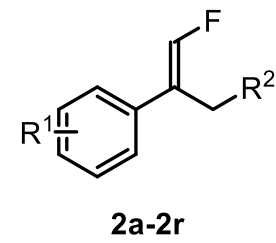

A dry tube under inert atmosphere was loaded with $1(0.3 \mathrm{mmol})$ and freshly distilled tetrahydrofuran $(1 \mathrm{~mL})$. In a second tube $\mathrm{LiAlH}_{4}(0.3 \mathrm{mmol}, 95 \%)$ was mixed with tetrahydrofuran $(2 \mathrm{~mL})$ and this suspension was slowly added to the reaction mixture at room temperature. It was then allowed to stir at room temperature or $70^{\circ} \mathrm{C}$ until completion of the reaction (6-24 h). The reaction was carefully quenched with a $30 \%$ potassium sodium tartrate solution $(5 \mathrm{~mL})$ at room temperature and the resulting mixture was stirred for 30 minutes. The mixture was diluted with diethyl ether $(10 \mathrm{~mL})$ and the aqueous layer was extracted with diethyl ether $(3 \times 10 \mathrm{~mL})$. The combined organic layers were dried over $\mathrm{Na}_{2} \mathrm{SO}_{4}$, filtered off and concentrated under reduced pressure. The residue was finally purified by column chromatography on silica gel to afford the product $\mathbf{2}$.

Note: 4-nitrofluorobenzene was used as an internal standard for ${ }^{19} \mathrm{~F} N \mathrm{MR}$ yields.

\section{Procedure for the reduction of $\alpha-\mathrm{CF}_{3}$ styrenes 1s-1x:}<smiles></smiles>

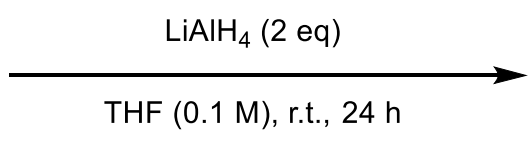

$\operatorname{THF}(0.1 \mathrm{M})$, r.t., $24 \mathrm{~h}$<smiles>[R]C/C(=C/F)c1cc[R1]cc1</smiles>

A dry tube under inert atmosphere was loaded with $1(0.3 \mathrm{mmol})$ and freshly distilled tetrahydrofuran $(1 \mathrm{~mL})$. In a second tube $\mathrm{LiAlH}_{4}(0.6 \mathrm{mmol}, 95 \%)$ was mixed with tetrahydrofuran $(2 \mathrm{~mL})$ and this suspension was slowly added to the reaction mixture at room temperature. It was then allowed to stir at room temperature for 24 hours. The reaction was carefully quenched with a $30 \%$ potassium sodium tartrate solution $(5 \mathrm{~mL})$ at room temperature and the resulting mixture was stirred for 30 minutes. The mixture was diluted with diethyl ether $(10 \mathrm{~mL})$ and the aqueous layer was extracted with diethyl ether $(3 \times 10 \mathrm{~mL})$. 
The combined organic layers were dried over $\mathrm{Na}_{2} \mathrm{SO}_{4}$, filtered off and concentrated under reduced pressure. The residue was finally purified by column chromatography on silica gel to afford the product 2.

General procedure for the reduction of $\beta-\mathrm{CF}_{3}$ acrylates $1 \mathbf{u}^{\prime}$ and $\mathbf{1 y}$-1aj:<smiles>CCOC(=O)C=C(OCC)c1cc[R1]cc1</smiles>

$1 u^{\prime}, 1 y-1$ aj

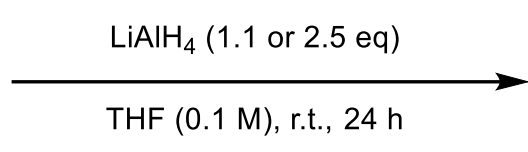

$\operatorname{THF}(0.1 \mathrm{M})$, r.t., $24 \mathrm{~h}$<smiles>OCCC(=CF)c1ccncc1</smiles>

$2 u, 2 y-2 a j$

A dry tube under inert atmosphere was loaded with $1(0.3 \mathrm{mmol})$ and freshly distilled tetrahydrofuran $(1 \mathrm{~mL})$. In a second tube $\mathrm{LiAlH}_{4}(0.75 \mathrm{mmol}, 95 \%)$ was mixed with tetrahydrofuran $(2 \mathrm{~mL})$ and this suspension was slowly added to the reaction mixture at room temperature. It was then allowed to stir at room temperature for 24 hours. The reaction was carefully quenched with a $30 \%$ potassium sodium tartrate solution $(5 \mathrm{~mL})$ at room temperature and the resulting mixture was stirred for 30 minutes. The mixture was diluted with diethyl ether $(10 \mathrm{~mL})$ and the aqueous layer was extracted with diethyl ether $(3 \times 10 \mathrm{~mL})$. The combined organic layers were dried over $\mathrm{Na}_{2} \mathrm{SO}_{4}$, filtered off and concentrated under reduced pressure. The residue was finally purified by column chromatography on silica gel to afford the product 2 .

\section{Procedure for the reduction of tetrasubstituted $-\mathrm{CF}_{3}$ alkene 1ak:}<smiles>CCOC(=O)C(C)=C(c1ccccc1)C(F)(F)F</smiles>

$1 \mathrm{ak}$

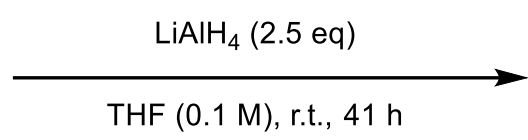

THF (0.1 M), r.t., $41 \mathrm{~h}$<smiles>CC(CO)C(=CF)c1ccccc1</smiles>

2ak

A dry tube under inert atmosphere was loaded with 1ak $(0.3 \mathrm{mmol})$ and freshly distilled tetrahydrofuran $(1 \mathrm{~mL})$. In a second tube $\mathrm{LiAlH}_{4}(0.75 \mathrm{mmol}, 95 \%)$ was mixed with tetrahydrofuran $(2 \mathrm{~mL})$ and this suspension was slowly added to the reaction mixture at room temperature. It was then allowed to stir at room temperature for 41 hours. The reaction was carefully quenched with a $30 \%$ potassium sodium tartrate solution $(5 \mathrm{~mL})$ at room temperature and the resulting mixture was stirred for 30 minutes. The mixture was diluted with diethyl ether $(10 \mathrm{~mL})$ and the aqueous layer was extracted with diethyl ether $(3 \times 10 \mathrm{~mL})$. The combined organic layers were dried over $\mathrm{Na}_{2} \mathrm{SO}_{4}$, filtered off and concentrated under reduced pressure. The residue was finally purified by column chromatography on silica gel to afford the product 2 ak. 


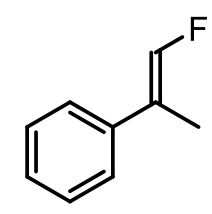

$2 a$

(E)-(1-fluoroprop-1-en-2-yl)benzene 2a: obtained as a colorless liquid $\left(78 \%{ }^{19} \mathrm{~F}\right.$ NMR yield, $56 \%$ isolated yield, $67 \mathrm{mg}$, d.r. = 95:5) after column chromatography on silica gel (100\% pentane). Rf: 0.70 (100\% pentane); ${ }^{1} \mathbf{H}$ NMR ( $\left.300 \mathrm{MHz}, \mathrm{CDCl}_{3}\right)$ : 6 7.39-7.27 (m, 5H), 7.05-6.76 (m, 1H), 2.05-2.04 (m, 3H) ppm; ${ }^{13} \mathrm{C} \mathrm{NMR}(75 \mathrm{MHz}$, $\left.\mathrm{CDCl}_{3}\right): \delta 146.2(\mathrm{~d}, J=256.5 \mathrm{~Hz}), 137.8(\mathrm{~d}, J=9.0 \mathrm{~Hz}), 128.8,127.6(\mathrm{~d}, J=0.8 \mathrm{~Hz})$, $126.1(\mathrm{~d}, J=3.8 \mathrm{~Hz}), 120.3(\mathrm{~d}, J=9.8 \mathrm{~Hz}), 12.5(\mathrm{~d}, J=6.0 \mathrm{~Hz}) \mathrm{ppm} ;{ }^{19} \mathrm{~F}$ NMR $(282$ $\mathrm{MHz}, \mathrm{CDCl}_{3}$ ): $\delta$-131.7 ppm; IR (neat): v 2955, 2923, 2854, 1741, 1461, 1377, 1232, 1155, 860 $\mathrm{cm}^{-1}$; HRMS $(\mathrm{Cl}+) \mathrm{m} / \mathrm{z}:[\mathrm{M}]^{+} \mathrm{Calcd}$ for $\mathrm{C}_{9} \mathrm{H}_{9} \mathrm{~F}$ 136.0688; Found 136.0689 .

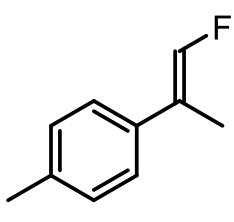

2b

(E)-1-(1-fluoroprop-1-en-2-yl)-4-methylbenzene $\mathbf{2 b}$ : obtained as a colorless liquid (94\% ${ }^{19} \mathrm{~F} \mathrm{NMR}$ yield, $58 \%$ isolated yield, $47 \mathrm{mg}$, d.r. = 94:6) after column chromatography on silica gel (100\% pentane). Rf: 0.70 (100\% pentane); ${ }^{1} \mathbf{H}$ NMR $\left(300 \mathrm{MHz} \mathrm{CDCl}_{3}\right): \delta$ 7.21-7.13 $(\mathrm{m}, 4 \mathrm{H}), 6.88(\mathrm{~d}, J=84.0 \mathrm{~Hz}, 1 \mathrm{H}), 2.34(\mathrm{~s}$, $3 \mathrm{H}), 2.03-2.01(\mathrm{~m}, 3 \mathrm{H}) \mathrm{ppm} ;{ }^{13} \mathrm{C}$ NMR $\left(75 \mathrm{MHz}, \mathrm{CDCl}_{3}\right): \delta 145.7$ (d, $J=255.0$ $\mathrm{Hz}), 137.3(\mathrm{~d}, J=1.5 \mathrm{~Hz}), 134.7(\mathrm{~d}, J=8.3 \mathrm{~Hz}), 129.4,125.9(\mathrm{~d}, J=3.0 \mathrm{~Hz}), 120.0$ (d, $J=9.8 \mathrm{~Hz}), 21.2,12.4(\mathrm{~d}, J=6.0 \mathrm{~Hz}) \mathrm{ppm} ;{ }^{19} \mathrm{~F}$ NMR $\left(282 \mathrm{MHz}, \mathrm{CDCl}_{3}\right): \delta-132.8 \mathrm{ppm}$; IR (neat): v 2959, 2925, 1665, 1515, 1118, 1064, 805, $539 \mathrm{~cm}^{-1}$; HRMS (Cl+) m/z: [M] Calcd for $\mathrm{C}_{10} \mathrm{H}_{11} \mathrm{~F}$ 150.0845; Found 150.0837.

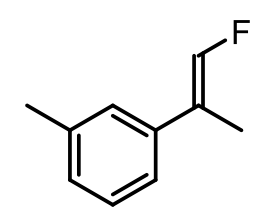

2c

(E)-1-(1-fluoroprop-1-en-2-yl)-3-methylbenzene 2c: obtained as a light yellow liquid ( $86 \%{ }^{19} \mathrm{~F}$ NMR yield, $49 \%$ isolated yield, $39 \mathrm{mg}$, d.r. = 95:5) after column chromatography on silica gel (100\% pentane). Rf: 0.68 (100\% pentane); ${ }^{1} \mathbf{H}$ NMR $\left(300 \mathrm{MHz}, \mathrm{CDCl}_{3}\right): \delta 7.23-7.20(\mathrm{~m}, 1 \mathrm{H}), 7.11-7.09(\mathrm{~m}, 3 \mathrm{H})$, 7.04-6.74 (m, 1H), $2.36(\mathrm{~s}, 3 \mathrm{H}), 2.04-2.02(\mathrm{~m}, 3 \mathrm{H}) \mathrm{ppm} ;{ }^{13} \mathrm{C}$ NMR $(75 \mathrm{MHz}$, $\left.\mathrm{CDCl}_{3}\right): \delta 146.0(\mathrm{~d}, J=255.8 \mathrm{~Hz}), 138.3,137.7(\mathrm{~d}, J=8.3 \mathrm{~Hz}), 128.6,128.3(\mathrm{~d}, J$ $=0.8 \mathrm{~Hz}), 126.8(\mathrm{~d}, J=3.0 \mathrm{~Hz}), 123.1(\mathrm{~d}, J=3.0 \mathrm{~Hz}), 120.2(\mathrm{~d}, J=9.0 \mathrm{~Hz}), 21.6,12.4(\mathrm{~d}, J=6.0$ $\mathrm{Hz}$ ) ppm; ${ }^{19} \mathrm{~F}$ NMR $\left(282 \mathrm{MHz}, \mathrm{CDCl}_{3}\right): \delta-132.0$ ppm; IR (neat): v 3036, 2925, 2863, 1666, 1446, 1116, 1077, 1065, 780, $645 \mathrm{~cm}^{-1}$; HRMS (Cl+) m/z: [M]+ Calcd for $\mathrm{C}_{10} \mathrm{H}_{11} \mathrm{~F}$ 150.0845; Found 150.0848 .

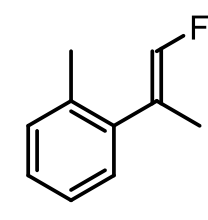

2d

(E)-1-(1-fluoroprop-1-en-2-yl)-2-methylbenzene $2 \mathrm{~d}$ : obtained as a light yellow liquid (92\% ${ }^{19} \mathrm{~F}$ NMR yield, $42 \%$ isolated yield, $34 \mathrm{mg}$, d.r. = 82:18) after column chromatography on silica gel (100\% pentane). Rf: 0.68 (100\% pentane); ${ }^{\mathbf{1}} \mathbf{H}$ NMR (300 MHz, $\mathrm{CDCl}_{3}$ ): $\delta 7.33(\mathrm{~s}, 1 \mathrm{H}), 7.22-7.15(\mathrm{~m}, 3 \mathrm{H}), 6.57(\mathrm{~d}, J=84.0 \mathrm{~Hz}, 1 \mathrm{H}), 2.37$ (s, 3H), 2.01-2.00 (m, 3H) ppm; ${ }^{13} \mathrm{C}$ NMR $\left(75 \mathrm{MHz}, \mathrm{CDCl}_{3}\right): \delta 146.2$ (d, $J=257.3$ $\mathrm{Hz}), 137.6(\mathrm{~d}, J=9.8 \mathrm{~Hz}), 130.4,129.6(\mathrm{~d}, J=3.0 \mathrm{~Hz}), 128.9,127.8,125.9,120.2$ (d, $J=8.3 \mathrm{~Hz}), 19.8,14.6(\mathrm{~d}, J=5.3 \mathrm{~Hz}) \mathrm{ppm} ;{ }^{19} \mathrm{~F} \mathbf{N M R}\left(282 \mathrm{MHz}, \mathrm{CDCl}_{3}\right): \delta-131.1 \mathrm{ppm}$; IR 
(neat): v 3066, 3020, 2926, 2861, 1674, 1457, 1108, 1061, 758, 725, $455 \mathrm{~cm}^{-1}$; HRMS (Cl+) m/z: $[\mathrm{M}]^{+}$Calcd for $\mathrm{C}_{10} \mathrm{H}_{11} \mathrm{~F}$ 150.0845; Found 150.0845 .<smiles>C/C(=C\F)c1ccc(C(C)C)cc1</smiles>

$2 e$

(E)-1-(1-fluoroprop-1-en-2-yl)-4-isopropylbenzene 2e: obtained as a colorless liquid (98\% ${ }^{19} \mathrm{~F}$ NMR yield, $80 \%$ isolated yield, $67 \mathrm{mg}$, d.r. = 93:7) after column chromatography on silica gel (100\% pentane). Rf: 0.76 (100\% pentane); ${ }^{1} \mathbf{H}$ NMR (300 MHz, $\left.\mathrm{CDCl}_{3}\right): \delta$ 7.25-7.18 (m, 4H), 7.04-6.74 (m, 1H), $2.90(\mathrm{~h}, J=7.0 \mathrm{~Hz}, 1 \mathrm{H}), 2.04-2.02(\mathrm{~m}, 3 \mathrm{H}), 1.26-1.24(\mathrm{~m}, 6 \mathrm{H}) \mathrm{ppm} ;{ }^{13} \mathrm{C} \mathbf{N M R}$ $\left(75 \mathrm{MHz}, \mathrm{CDCl}_{3}\right): \delta 148.3(\mathrm{~d}, J=1.5 \mathrm{~Hz}), 145.8(\mathrm{~d}, J=255.8 \mathrm{~Hz}), 135.1$ (d, $J=$ $4.5 \mathrm{~Hz}$ ), 126.8, 126.0 (d, $J=3.8 \mathrm{~Hz}$ ), 120.0 (d, $J=9.8 \mathrm{~Hz}$ ), 33.9, 24.1, 12.4, $12.3 \mathrm{ppm} ;{ }^{19} \mathrm{~F} \mathrm{NMR}$ (282 MHz, CDCl $)$ ): $\delta$-132.7 ppm; IR (neat): v 3090, 2961, 2928, 2871, 1664, 1461, 1118, 1065, 836, 813, $564 \mathrm{~cm}^{-1}$; HRMS (Cl+) m/z: [M] ${ }^{+}$Calcd for $\mathrm{C}_{12} \mathrm{H}_{15} \mathrm{~F}$ 178.1158; Found 178.1163.<smiles>C/C(=C\F)c1ccc(-c2ccccc2)cc1</smiles>

$2 f$

(E)-4-(1-fluoroprop-1-en-2-yl)-1,1'-biphenyl 2f: obtained as a white amorphous solid $(74 \%$ yield, $63 \mathrm{mg}$, d.r. = 93:7) after column chromatography on silica gel (100\% pentane). Rf: 0.75 (cyclohexane:Et ${ }_{2} \mathrm{O}$, 90:10); ${ }^{1} \mathbf{H}$ NMR (300 MHz, $\left.\mathrm{CDCl}_{3}\right): \delta$ 7.61-7.56 (m, 4H), 7.47-7.33 (m, 5H), $6.98(\mathrm{~d}, J=87.0 \mathrm{~Hz}, 1 \mathrm{H}), 2.09-2.08(\mathrm{~m}, 3 \mathrm{H}) \mathrm{ppm} ;{ }^{13} \mathrm{C} \mathrm{NMR}\left(75 \mathrm{MHz}, \mathrm{CDCl}_{3}\right)$ : $\delta 146.2(\mathrm{~d}, J=256.5 \mathrm{~Hz}), 140.7,140.4(\mathrm{~d}, J=1.5 \mathrm{~Hz}), 136.6(\mathrm{~d}, J=9.0 \mathrm{~Hz})$, 129.0, 127.5, 127.4, 127.1, $126.3(\mathrm{~d}, J=3.8 \mathrm{~Hz}), 119.8$ (d, $J=10.5 \mathrm{~Hz}), 12.3(\mathrm{~d}, J=6.0 \mathrm{~Hz}$ ) ppm; ${ }^{19}$ F NMR (282 MHz, CDCl 3 ): $\delta$-131.4 ppm; IR (neat): v 3037, 2997, 2924, 1655, 1409, 1115, 1065, 819, 760, 719, 689, $444 \mathrm{~cm}^{-1}$; HRMS (El+) m/z: [M] ${ }^{+}$Calcd for $\mathrm{C}_{15} \mathrm{H}_{13} \mathrm{~F}$ 212.1001; Found 212.1002 .<smiles>CC(=CF)c1ccc2ccccc2c1</smiles>

$2 \mathrm{~g}$

(E)-2-(1-fluoroprop-1-en-2-yl)naphthalene 2g: obtained as a white amorphous solid (76\% yield, $63 \mathrm{mg}$, d.r. $=93: 7)$ after column chromatography on silica gel (100\% pentane). $\mathbf{R f :} 0.78$ (cyclohexane:Et ${ }_{2} \mathrm{O}$, 90:10); ${ }^{1} \mathrm{H}$ NMR (300 MHz, $\left.\mathrm{CDCl}_{3}\right): \delta$ 7.87-7.81 (m, 3H), $7.77(\mathrm{~s}, 1 \mathrm{H}), 7.55-$ $7.46(\mathrm{~m}, 3 \mathrm{H}), 7.24-6.95(\mathrm{~m}, 1 \mathrm{H}), 2.20-2.18(\mathrm{~m}, 3 \mathrm{H}) \mathrm{ppm} ;{ }^{13} \mathrm{C} \mathrm{NMR}(75 \mathrm{MHz}$, $\left.\mathrm{CDCl}_{3}\right): \delta 146.6(\mathrm{~d}, J=256.5 \mathrm{~Hz}), 135.0(\mathrm{~d}, J=8.3 \mathrm{~Hz}), 133.6,132.8,128.3$, 128.0, 127.7, 126.5, 126.0, 124.7 (d, J = 4.5 Hz), 124.0 (d, J=2.3 Hz), 120.1 (d, J = 9.8 Hz), 12.3 $(\mathrm{d}, J=6.8 \mathrm{~Hz}) \mathrm{ppm} ;{ }^{19} \mathrm{~F}$ NMR $\left(282 \mathrm{MHz}, \mathrm{CDCl}_{3}\right): \delta-130.9$ ppm; IR (neat): v 3057, 2924, 1655, 1594, 1384, 1106, 1069, 1057, 860, 810, 747, 730, 480, $472 \mathrm{~cm}^{-1}$; HRMS (El+) m/z: [M] ${ }^{+}$Calcd for $\mathrm{C}_{13} \mathrm{H}_{11} \mathrm{~F}$ 186.0845; Found 186.0845 . 
<smiles>COc1ccc(/C(C)=C/F)cc1</smiles>

$2 \mathrm{~h}$

(E)-1-(1-fluoroprop-1-en-2-yl)-4-methoxybenzene $\mathbf{2 h}$ : obtained as a light yellow liquid (99\% ${ }^{19} \mathrm{~F}$ NMR yield, $87 \%$ isolated yield, $71 \mathrm{mg}$, d.r. = 95:5) after column chromatography on silica gel (pentane: $\mathrm{Et}_{2} \mathrm{O}$, from 100:0 to 80:20). Rf: 0.21 (100\% pentane); ${ }^{1} \mathrm{H}$ NMR (300 MHz, $\left.\mathrm{CDCl}_{3}\right): \delta$ 7.25-7.21 (m, $2 \mathrm{H}), 7.00-6.70(\mathrm{~m}, 1 \mathrm{H}), 6.90-6.85(\mathrm{~m}, 2 \mathrm{H}), 3.81(\mathrm{~s}, 3 \mathrm{H}), 2.02-2.00(\mathrm{~m}, 3 \mathrm{H})$ ppm; $\left.{ }^{13} \mathrm{C} \mathrm{NMR} \mathrm{(75} \mathrm{MHz,} \mathrm{CDCl} 3\right): \delta 159.2,145.3$ (d, $\left.J=255.0 \mathrm{~Hz}\right), 130.0$ (d, J $=8.3 \mathrm{~Hz}), 127.1(\mathrm{~d}, J=3.8 \mathrm{~Hz}), 119.6(\mathrm{~d}, J=9.8 \mathrm{~Hz}), 114.1,55.4,12.5(\mathrm{~d}, J=6.0 \mathrm{~Hz}) \mathrm{ppm} ;{ }^{19} \mathrm{~F}$ NMR (282 MHz, CDCl 3 ): $\delta$-133.6 ppm; IR (neat): v 3000, 2957, 2838, 1664, 1608, 1513, 1245, 1109, 1032, 832, 806, $587 \mathrm{~cm}^{-1}$; HRMS (EI+) m/z: [M] ${ }^{+}$Calcd for $\mathrm{C}_{10} \mathrm{H}_{11} \mathrm{FO}$ 166.0794; Found 166.0794 .<smiles>COc1cccc(/C(C)=C/F)c1</smiles>

$2 \mathbf{i}$

(E)-1-(1-fluoroprop-1-en-2-yl)-3-methoxybenzene 2i: obtained as a light yellow liquid (90\% ${ }^{19} \mathrm{~F}$ NMR yield, $85 \%$ isolated yield, $69 \mathrm{mg}$, d.r. = 95:5) after column chromatography on silica gel (pentane:Et ${ }_{2} \mathrm{O}$, from 100:0 to 80:20). Rf: 0.24 (100\% pentane); ${ }^{1} \mathbf{H}$ NMR (300 MHz, $\mathrm{CDCl}_{3}$ ): $\delta$ 7.28-7.25 $(\mathrm{m}, 1 \mathrm{H}), 7.06-6.76(\mathrm{~m}, 1 \mathrm{H}), 6.92-6.88(\mathrm{~m}, 1 \mathrm{H}), 6.84-6.81(\mathrm{~m}, 2 \mathrm{H}), 3.82(\mathrm{~s}$, $3 \mathrm{H})$, 2.04-2.02 (m, 3H) ppm; ${ }^{13} \mathrm{C}$ NMR $\left(75 \mathrm{MHz}, \mathrm{CDCl}_{3}\right): \delta 159.8,146.3$ (d, J $=256.5 \mathrm{~Hz}$ ), $139.2(\mathrm{~d}, J=9.0 \mathrm{~Hz}), 129.7,120.3(\mathrm{~d}, J=9.8 \mathrm{~Hz}), 118.5(\mathrm{~d}, J=3.0 \mathrm{~Hz}), 112.6$ (d, J = $0.8 \mathrm{~Hz}), 112.1$ (d, $J=3.0 \mathrm{~Hz}), 55.3,12.4(\mathrm{~d}, J=5.3 \mathrm{~Hz}) \mathrm{ppm} ;{ }^{19} \mathrm{~F} \mathrm{NMR}\left(282 \mathrm{MHz}, \mathrm{CDCl}_{3}\right): \delta-131.5$ ppm; IR (neat): v 3006, 2956, 2837, 1664, 1601, 1578, 1428, 1291, 1217, 1115, 1045, 775, 693 $\mathrm{cm}^{-1} ;$ HRMS $(\mathrm{Cl}+) \mathrm{m} / \mathrm{z}$ : [M] $]^{+}$Calcd for $\mathrm{C}_{10} \mathrm{H}_{11} \mathrm{FO}$ 166.0794; Found 166.0801 .<smiles>C/C(=C\F)c1ccc(N(C)C)cc1</smiles>

$2 \mathbf{j}$

(E)-4-(1-fluoroprop-1-en-2-yl)- $\mathbf{N}, \mathbf{N}$-dimethylaniline $\mathbf{2 j}$ : obtained as a light yellow amorphous solid (99\% yield, $69 \mathrm{mg}$, d.r. $=95: 5)$ after column chromatography on silica gel (pentane: $\mathrm{Et}_{2} \mathrm{O}$, from 100:0 to 80:20). Rf: 0.85 (pentane:Et $\left.{ }_{2} \mathrm{O}, 70: 30\right) ;{ }^{1} \mathbf{H}$ NMR $\left(300 \mathrm{MHz}, \mathrm{CDCl}_{3}\right): \delta$ 7.21-7.18 (m, 2H), 7.00 (s, 0.5H), 6.72-6.69 (m, 2.5H), $2.95(\mathrm{~s}, 3 \mathrm{H}), 2.01-2.00(\mathrm{~m}, 3 \mathrm{H}) \mathrm{ppm} ;{ }^{13} \mathrm{C}$ NMR $\left(75 \mathrm{MHz}, \mathrm{CDCl}_{3}\right): \delta 150.1,144.7(\mathrm{~d}, J=252.8 \mathrm{~Hz}), 126.7(\mathrm{~d}, J=3.0 \mathrm{~Hz}), 125.6$ (d, $J=8.3 \mathrm{~Hz}), 119.7(\mathrm{~d}, J=9.8 \mathrm{~Hz}), 112.7,40.7,12.3(\mathrm{~d}, J=6.0 \mathrm{~Hz}) \mathrm{ppm} ;{ }^{19} \mathrm{~F}$ NMR $(282 \mathrm{MHz}$, $\mathrm{CDCl}_{3}$ ): $\delta$-135.7 ppm; IR (neat): v 3102, 3048, 2924, 2804, 1653, 1609, 1520, 1350, 1229, 1101, 1062, 940, 821, 796, 571, $481 \mathrm{~cm}^{-1}$; HRMS (Cl+) m/z: [M] ${ }^{+}$Calcd for $\mathrm{C}_{11} \mathrm{H}_{14} \mathrm{FN}$ 179.1110; Found 179.1115 .<smiles>C/C(=C\F)c1ccc(C(F)(F)F)cc1</smiles>

2k

(E)-1-(1-fluoroprop-1-en-2-yl)-4-(trifluoromethyl)benzene 2k: obtained as a yellow liquid (94\% ${ }^{19} \mathrm{~F}$ NMR yield, $58 \%$ isolated yield, $50 \mathrm{mg}$, d.r. $=95: 5$ ) after column chromatography on silica gel (100\% pentane). Rf: $0.58(100 \%$ pentane); ${ }^{1} \mathrm{H}$ NMR $\left(300 \mathrm{MHz}, \mathrm{CDCl}_{3}\right): \delta 7.60-7.57(\mathrm{~m}, 2 \mathrm{H}), 7.42-7.40(\mathrm{~m}, 2 \mathrm{H})$, 7.09-6.81 (m, 1H), 2.07-2.05 (m, 3H) ppm; $\left.{ }^{13} \mathrm{C} \mathrm{NMR} \mathrm{(75} \mathrm{MHz,} \mathrm{CDCl}_{3}\right): \delta$ $147.1(\mathrm{~d}, J=259.5 \mathrm{~Hz}), 141.4(\mathrm{~d}, J=7.5 \mathrm{~Hz}), 129.6(\mathrm{qd}, J=32.3 \& 1.5 \mathrm{~Hz})$, $128.2(\mathrm{~d}, J=6.0 \mathrm{~Hz}), 126.2(\mathrm{~d}, J=3.0 \mathrm{~Hz}), 125.7$ (q, $J=4.0 \mathrm{~Hz}), 124.3$ (q, J = $270.0 \mathrm{~Hz}$ ), 12.2 (d, $J=6.0 \mathrm{~Hz}$ ) ppm; ${ }^{19} \mathrm{~F} \mathrm{NMR}\left(282 \mathrm{MHz}, \mathrm{CDCl}_{3}\right): \delta-63.1(3 \mathrm{~F}),-128.5 \mathrm{ppm}$; IR (neat): v 2930, 1664, 
1618, 1413, 1323, 1166, 1112, 1073, 845, $821 \mathrm{~cm}^{-1}$; HRMS (Cl+) m/z: [M] ${ }^{+}$Calcd for $\mathrm{C}_{10} \mathrm{H}_{8} \mathrm{~F}_{4}$ 204.0562; Found 204.0556.

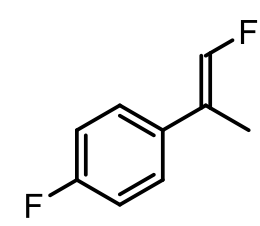

21

(E)-1-fluoro-4-(1-fluoroprop-1-en-2-yl)benzene 2l: obtained as a colorless liquid (83\% ${ }^{19} \mathrm{~F}$ NMR yield, 33\% isolated yield, $27 \mathrm{mg}$, d.r. = 95:5) after column chromatography on silica gel (100\% pentane). Rf: 0.61 (100\% pentane); ${ }^{1} \mathbf{H}$ NMR $\left(300 \mathrm{MHz}, \mathrm{CDCl}_{3}\right): \delta$ 7.29-7.23 $(\mathrm{m}, 2 \mathrm{H}), 7.06-6.98(\mathrm{~m}, 2.5 \mathrm{H}), 6.71-6.70$ $(\mathrm{m}, 0.5 \mathrm{H}), 2.03-2.01(\mathrm{~m}, 3 \mathrm{H}) \mathrm{ppm} ;{ }^{13} \mathrm{C} \mathrm{NMR}\left(75 \mathrm{MHz}, \mathrm{CDCl}_{3}\right): \delta 162.4(\mathrm{dd}, J=$ $244.9 \& 1.1 \mathrm{~Hz}), 145.9(\mathrm{dd}, J=256.1 \& 1.1 \mathrm{~Hz}), 133.7$ (dd, $J=9.0 \& 3.0 \mathrm{~Hz}$ ), 127.6 (dd, $J=7.9 \& 3.0 \mathrm{~Hz}$ ), $119.4(\mathrm{~d}, J=10.5 \mathrm{~Hz}), 115.6$ (d, $J=21.0 \mathrm{~Hz}), 12.6(\mathrm{~d}, J=6.0 \mathrm{~Hz})$ ppm; ${ }^{19}$ F NMR (282 MHz, CDCl 3 ): $\delta-115.6,-131.5$ ppm; IR (neat): v 2923, 2955, 2855, 1729, 1668, 1512, 1233, 1123, 1064, 838, 816, $578 \mathrm{~cm}^{-1}$; HRMS (Cl+) m/z: [M] $]^{+}$Calcd for $\mathrm{C}_{9} \mathrm{H}_{8} \mathrm{~F}_{2}$ 154.0594; Found 154.0590.<smiles>C/C(=C\F)c1ccc(Br)cc1</smiles>

$2 \mathrm{~m}$

(E)-1-bromo-4-(1-fluoroprop-1-en-2-yl)benzene $2 \mathrm{~m}$ : obtained as a light yellow amorphous solid ( $87 \%{ }^{19} \mathrm{~F}$ NMR yield, $68 \%$ isolated yield, $58 \mathrm{mg}$, d.r. $=95: 5)$ after column chromatography on silica gel (100\% pentane). Rf: 0.62 (100\% pentane); ${ }^{1} \mathbf{H}$ NMR (300 MHz, $\left.\mathrm{CDCl}_{3}\right): \delta$ 7.48-7.43 (m, 2H), 7.19-7.15 (m, 2H), 7.04-6.75 (m, 1H), 2.02-2.01 (m, 3H) ppm; ${ }^{13} \mathrm{C} \mathrm{NMR}\left(75 \mathrm{MHz}, \mathrm{CDCl}_{3}\right)$ : $\delta 146.3(\mathrm{~d}, J=258.0 \mathrm{~Hz}), 136.6(\mathrm{~d}, J=9.0 \mathrm{~Hz}), 131.8,127.6(\mathrm{~d}, J=3.0 \mathrm{~Hz})$, $121.4(\mathrm{~d}, J=1.5 \mathrm{~Hz}), 119.4(\mathrm{~d}, J=10.5 \mathrm{~Hz}), 12.3(\mathrm{~d}, J=6.0 \mathrm{~Hz})$ ppm; ${ }^{19} \mathrm{~F}$ NMR $\left(282 \mathrm{MHz}, \mathrm{CDCl}_{3}\right)$ : $\delta$-130.4 ppm; IR (neat): v 2959, 2925, 2858, 1661, 1489, 1124, 1077, 1008, 811, $486 \mathrm{~cm}^{-1}$; HRMS $(\mathrm{Cl}+) \mathrm{m} / \mathrm{z}$ : [M] ${ }^{+}$Calcd for $\mathrm{C}_{9} \mathrm{H}_{8} \mathrm{BrF}$ 213.9793; Found 213.9794.<smiles>C/C(=C\F)c1cccc(Cl)c1</smiles>

$2 n$

(E)-1-chloro-3-(1-fluoroprop-1-en-2-yl)benzene 2 n: obtained as a colorless liquid (94\% ${ }^{19} \mathrm{~F}$ NMR yield, $65 \%$ isolated yield, $53 \mathrm{mg}$, d.r. $\left.=94: 6\right)$ after column chromatography on silica gel (100\% pentane). Rf: $0.61(100 \%$ pentane); ${ }^{1} \mathbf{H}$ NMR (300 MHz, $\left.\mathrm{CDCl}_{3}\right): \delta$ 7.28-7.16 (m, 4H), 7.05-6.76 (m, 1H), 2.03-2.01 (m, 3H) ppm; $\left.{ }^{13} \mathrm{C} \mathrm{NMR} \mathrm{(75} \mathrm{MHz,} \mathrm{CDCl}_{3}\right): \delta 146.7$ (d, $\left.J=258.0 \mathrm{~Hz}\right)$, $139.6(\mathrm{~d}, J=9.0 \mathrm{~Hz}), 134.6,129.9,127.6(\mathrm{~d}, J=1.5 \mathrm{~Hz}), 126.2,124.1(\mathrm{~d}, J=$ $3.8 \mathrm{~Hz}$ ), 119.3 (d, $J=10.5 \mathrm{~Hz}$ ), 12.2 (d, $J=6.0 \mathrm{~Hz})$ ppm; ${ }^{19} \mathrm{~F} \mathrm{NMR}\left(282 \mathrm{MHz}, \mathrm{CDCl}_{3}\right): \delta-129.9$ ppm; IR (neat): v 3090, 2927, 1665, 1123, 1083, 1062, 811, 780, 700, $689 \mathrm{~cm}^{-1}$; HRMS (Cl+) $\mathrm{m} / \mathrm{z}:[\mathrm{M}]^{+}$Calcd for $\mathrm{C}_{9} \mathrm{H}_{8}{ }^{37} \mathrm{CIF}$ 172.0269; Found 172.0269 .

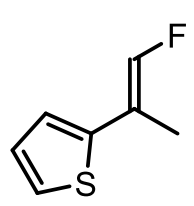

20

(E)-2-(1-fluoroprop-1-en-2-yl)thiophene 20: obtained as a colorless liquid (93\% ${ }^{19} \mathrm{~F}$ NMR yield, $44 \%$ isolated yield, $42 \mathrm{mg}$, d.r. $\left.=86: 14\right)$ after column chromatography on silica gel (100\% pentane). $\mathbf{R f :} 0.67$ (100\% pentane); ${ }^{\mathbf{1}} \mathbf{H} \mathbf{N M R}$ (300 MHz, $\left.\mathrm{CDCl}_{3}\right)$ : 8 7.23-6.94 (m, 1H), 7.16-7.14 (m, 1H), 7.00-6.98 (m, 2H), 2.07$2.05(\mathrm{~m}, 3 \mathrm{H}) \mathrm{ppm} ;{ }^{13} \mathrm{C} \mathrm{NMR}\left(75 \mathrm{MHz}, \mathrm{CDCl}_{3}\right): \delta 145.5(\mathrm{~d}, J=258.8 \mathrm{~Hz}), 140.4(\mathrm{~d}, J$ $=8.3 \mathrm{~Hz}), 127.4,123.6-123.5(\mathrm{~m}, 2 \mathrm{C}), 115.3(\mathrm{~d}, J=13.5 \mathrm{~Hz}), 12.8(\mathrm{~d}, J=6.0 \mathrm{~Hz})$ 
ppm; ${ }^{19}$ F NMR (282 MHz, CDCl $): \delta-132.9$ ppm; IR (neat): v 3080, 2924, 2855, 1659, 1438, $1225,1114,1055,850,805,692 \mathrm{~cm}^{-1}$; HRMS $(\mathrm{Cl}+) \mathrm{m} / \mathrm{z}$ : [M] ${ }^{+}$Calcd for $\mathrm{C}_{7} \mathrm{H}_{8} \mathrm{FS}$ 143.0331; Found 143.0337.

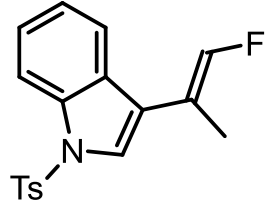

$2 p$

(E)-3-(1-fluoroprop-1-en-2-yl)-1-tosyl-1H-indole 2p: obtained as a white amorphous solid $(78 \%$ yield, $57 \mathrm{mg}$, d.r. $=99: 1)$ after column chromatography on silica gel (pentane: $\mathrm{Et}_{2} \mathrm{O}$, from 100:0 to 80:20). Rf: 0.42 (pentane: $\left.\mathrm{Et}_{2} \mathrm{O}, 80: 20\right) ;{ }^{1} \mathrm{H}$ NMR $\left(300 \mathrm{MHz}, \mathrm{CDCl}_{3}\right): \delta 8.03-7.99(\mathrm{~m}, 1 \mathrm{H}), 7.80-$ $7.77(\mathrm{~m}, 2 \mathrm{H}), 7.66-7.52(\mathrm{~m}, 2 \mathrm{H}), 7.37-7.22(\mathrm{~m}, 4.5 \mathrm{H}), 6.96-6.95(\mathrm{~m}, 0.5 \mathrm{H})$, $2.34(\mathrm{~s}, 3 \mathrm{H}), 2.08$ (dd, $J=3 \& 3 \mathrm{~Hz}) \mathrm{ppm} ;{ }^{13} \mathrm{C} \mathrm{NMR}\left(75 \mathrm{MHz}, \mathrm{CDCl}_{3}\right): \delta 146.8(\mathrm{~d}, J=256.5 \mathrm{~Hz})$, $145.2,135.6,135.2,130.1,128.7,127.0,125.1,123.6,123.4$ (d, $J=6.8 \mathrm{~Hz}$ ), 120.8, 120.0 (d, $J=$ $9.8 \mathrm{~Hz}), 114.0,113.0(\mathrm{~d}, J=12.0 \mathrm{~Hz}), 21.7,12.8(\mathrm{~d}, J=6.0 \mathrm{~Hz}) \mathrm{ppm} ;{ }^{19} \mathrm{~F} \mathrm{NMR}\left(282 \mathrm{MHz}, \mathrm{CDCl}_{3}\right)$ : $\delta$-129.9 ppm; IR (neat): v 3148, 2957, 2921, 2874, 1666, 1445, 1364, 1173, 1122, 1087, 741, 680, 666, 581, 566, $536 \mathrm{~cm}^{-1}$; HRMS (ES+) m/z: $\left[\mathrm{M}+\mathrm{NH}_{4}\right]^{+}$Calcd for $\mathrm{C}_{18} \mathrm{H}_{20} \mathrm{FN}_{2} \mathrm{O}_{2} \mathrm{~S}$ 347.1230; Found 347.1239.

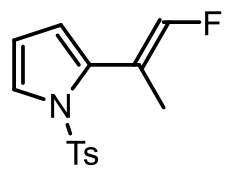

(E)-3-(1-fluoroprop-1-en-2-yl)-1-tosyl-1H-indole 2q: obtained as a mixture of non-separable diastereomers as a white amorphous solid ( $83 \%$ yield, $58 \mathrm{mg}$, d.r. $=88: 12$ ) after column chromatography on silica gel (pentane: $\mathrm{Et}_{2} \mathrm{O}$, from 100:0 to 90:0). Rf: 0.42 (pentane:Et $\left.{ }_{2} \mathrm{O}, 80: 20\right) ;{ }^{1} \mathbf{H}$ NMR (300 MHz, $\left.\mathrm{C}_{6} \mathrm{D}_{6}\right): \delta 7.63-$

$2 q \quad 7.59(\mathrm{~m}, 0.48 \mathrm{H}$, minor), 7.53-7.49 (m, 2H, major), 7.39-7.38 (m, 1.24H), $6.61(\mathrm{~d}$, $J=9.0 \mathrm{~Hz}, 0.48 \mathrm{H}$, minor), 6.53-6.49 (m, $2 \mathrm{H}$, major), 6.25-6.24 (m, 0.5H, major), 6.22-6.21 (m, $0.12 \mathrm{H}$, minor), 5.98-5.96 (m, 0.85H), $5.91(\mathrm{t}, J=3 \mathrm{~Hz}, 1 \mathrm{H}$, major), 5.83-5.82 (m, 0.24H, minor), 5.77-5.75 (m, $1 \mathrm{H}$, major), 2.02-2.01 (m, 3H, major), 1.78-1.76 (m, 0.72H, minor), 1.72-1.71 (m, 3.72H) ppm; ${ }^{13} \mathrm{C}$ NMR (75 MHz, $\left.\mathrm{C}_{6} \mathrm{D}_{6}\right): \delta 148.5$ (d, $J=264.8 \mathrm{~Hz}$ ), 146.6 (d, $J=258.8 \mathrm{~Hz}$ ), 144.7, 144.5, 136.7-136.6 (m, 2C), $131.8(\mathrm{~d}, J=13.5 \mathrm{~Hz}), 129.7,127.2-127.1(\mathrm{~m}, 2 \mathrm{C}), 125.0(\mathrm{~d}, J=0.8$ $\mathrm{Hz}), 124.3,121.0,116.3$ (d, $J=2.3 \mathrm{~Hz}), 115.7$ (d, $J=1.5 \mathrm{~Hz}$ ), 113.8, 113.4 (d, $J=14.3 \mathrm{~Hz}), 112.9$, $112.4,112.2,21.1,18.2(\mathrm{~d}, J=3.0 \mathrm{~Hz}), 15.5,15.4$ (d, $J=3.0 \mathrm{~Hz})$ ppm; ${ }^{19} \mathrm{~F}$ NMR $\left(282 \mathrm{MHz}, \mathrm{CDCl}_{3}\right)$ : $\delta$-123.1 (major), -123.6 (minor) ppm; HRMS (AP+) m/z: $[\mathrm{M}+\mathrm{H}]^{+}$Calcd for $\mathrm{C}_{14} \mathrm{H}_{15} \mathrm{FNO}_{2} \mathrm{~S}$ 280.0808; Found 280.0816.

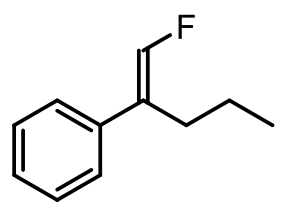

$2 r$

(E)-(1-fluoropent-1-en-2-yl)benzene 2r: obtained as a colorless liquid (93\% ${ }^{19} \mathrm{~F}$ NMR yield, $50 \%$ isolated yield, $33 \mathrm{mg}$, d.r. = 98:2) after column chromatography on silica gel (100\% pentane). Rf: 0.82 (100\% pentane); ${ }^{1} \mathbf{H}$ NMR (300 MHz, CDCl $): \delta 7.30-7.19(\mathrm{~m}, 5 \mathrm{H}), 6.70(\mathrm{~d}, J=84.0 \mathrm{~Hz}, 1 \mathrm{H}), 2.45-$ $2.40(\mathrm{~m}, 2 \mathrm{H}), 1.37-1.25(\mathrm{~m}, 2 \mathrm{H}), 0.80(\mathrm{t}, J=7.5 \mathrm{~Hz}, 3 \mathrm{H}) \mathrm{ppm} ;{ }^{13} \mathrm{C}$ NMR $(75$ $\mathrm{MHz}_{\mathrm{CDCl}}$ ): $\delta 146.0(\mathrm{~d}, J=256.5 \mathrm{~Hz}), 136.8(\mathrm{~d}, J=9.8 \mathrm{~Hz}), 128.7,127.5$, $127.0\left(\mathrm{~d}, J=3.0 \mathrm{~Hz}\right.$ ), $125.1(\mathrm{~d}, J=9.8 \mathrm{~Hz}), 28.6(\mathrm{~d}, J=4.5 \mathrm{~Hz}), 21.0(\mathrm{~d}, J=2.3 \mathrm{~Hz}), 13.8 \mathrm{ppm} ;{ }^{19} \mathrm{~F}$ NMR (282 MHz, CDCl $): \delta$-131.9 ppm; IR (neat): v 2963, 2920, 2851, 1463, 1260, 1016, 798 $\mathrm{cm}^{-1}$; HRMS $(\mathrm{Cl}+) \mathrm{m} / \mathrm{z}$ : [M] ${ }^{+}$Calcd for $\mathrm{C}_{11} \mathrm{H}_{13} \mathrm{~F}$ 164.1001; Found 164.0993. 


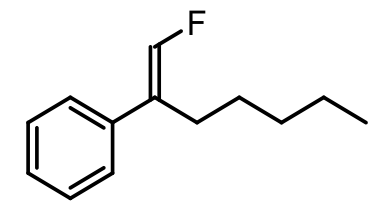

2s

(E)-(1-fluorohept-1-en-2-yl)benzene 2s: obtained as a colorless liquid $\left(96 \%{ }^{19} \mathrm{~F}\right.$ NMR yield, $70 \%$ isolated yield, $47 \mathrm{mg}$, d.r. = 96:4) after column chromatography on silica gel (100\% pentane). $\mathbf{R f :} 0.84$ (100\% pentane); ${ }^{1} \mathbf{H ~ N M R}\left(300 \mathrm{MHz}, \mathrm{CDCl}_{3}\right): \delta$ 7.39-7.26 $(\mathrm{m}, 5 \mathrm{H}), 6.78(\mathrm{~d}, \mathrm{~J}=87.0 \mathrm{~Hz}, 1 \mathrm{H})$, 2.55-2.50 (m, 2H), 1.43-1.27 (m, 6H), 0.87-0.83 (m, 3H) ppm; ${ }^{13} \mathrm{C}$ NMR $\left(75 \mathrm{MHz}, \mathrm{CDCl}_{3}\right): \delta 145.9(\mathrm{~d}, J=256.5 \mathrm{~Hz}), 136.8(\mathrm{~d}, J=9.0 \mathrm{~Hz}), 128.7$, 127.5, 126.9 (d, $J=3.0 \mathrm{~Hz}$ ), 125.4 (d, $J=9.0 \mathrm{~Hz}$ ), 31.6, 27.5 (d, $J=2.3 \mathrm{~Hz}), 26.6$ (d, $J=4.5 \mathrm{~Hz}$ ), 22.5, 14.2 ppm; ${ }^{19}$ F NMR (282 MHz, CDCl $)$ : $\delta$-132.0 ppm; IR (neat): v 2957, 2928, 2861, 1660, 1445, 1122, 1107, 758, $696 \mathrm{~cm}^{-1}$; HRMS $(\mathrm{Cl}+) \mathrm{m} / \mathrm{z}$ : [M] ${ }^{+}$Calcd for $\mathrm{C}_{13} \mathrm{H}_{17} \mathrm{~F}$ 192.1314; Found 192.1304.

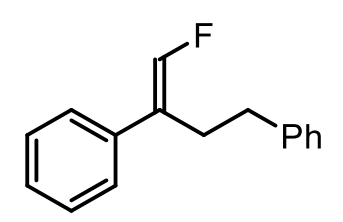

2t

(E)-(4-fluorobut-3-ene-1,3-diyl)dibenzene 2t: obtained as a light yellow oil ( $97 \%{ }^{19} \mathrm{~F}$ NMR yield, $65 \%$ isolated yield, $45 \mathrm{mg}$, d.r. = 96:4) after column chromatography on silica gel (pentane:Et ${ }_{2} \mathrm{O}$, from 100:0 to 95:5). Rf: 0.45 (100\% pentane); ${ }^{1} \mathrm{H}$ NMR (300 MHz, $\left.\mathrm{CDCl}_{3}\right): \delta$ 7.49-7.26 (m, 10H), 6.87 (d, $J=84.0 \mathrm{~Hz}, 1 \mathrm{H}), 2.99-2.93(\mathrm{~m}, 2 \mathrm{H}), 2.81-2.76(\mathrm{~m}, 2 \mathrm{H}) \mathrm{ppm} ;{ }^{13} \mathrm{C}$ NMR $(75$ $\left.\mathrm{MHz}, \mathrm{CDCl}_{3}\right): \delta 146.2(\mathrm{~d}, J=258.0 \mathrm{~Hz}), 141.7,136.4(\mathrm{~d}, J=6.0 \mathrm{~Hz}), 128.8$, 128.5, 128.4, 127.7, $127.0(\mathrm{~d}, J=3.0 \mathrm{~Hz}), 126.1,124.5(\mathrm{~d}, J=9.0 \mathrm{~Hz}), 34.2(\mathrm{~d}, J=2.3 \mathrm{~Hz}$ ), 28.8 $(\mathrm{d}, J=3.8 \mathrm{~Hz}) \mathrm{ppm} ;{ }^{19} \mathrm{~F}$ NMR $\left(282 \mathrm{MHz}, \mathrm{CDCl}_{3}\right): \delta-130.8 \mathrm{ppm}$; IR (neat): v 3028, 2928, 2864, 1660, 1495, 1454, 1118, 757, 741, $694 \mathrm{~cm}^{-1}$; HRMS (Cl+) m/z: [M] ${ }^{+}$Calcd for $\mathrm{C}_{16} \mathrm{H}_{15} \mathrm{~F} 226.1158$; Found 226.1160 .

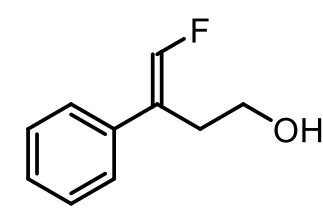

$2 \mathbf{u}$

(E)-4-fluoro-3-phenylbut-3-en-1-ol 2u: obtained as a light yellow liquid (72\% yield, $48 \mathrm{mg}$, d.r. $=81: 19$ ) after column chromatography on silica gel (pentane:Et ${ }_{2} \mathrm{O}$, from 100:0 to 50:50). Rf: 0.42 (pentane:Et $\left.{ }_{2} \mathrm{O}, 50: 50\right) ;{ }^{1} \mathbf{H}$ NMR $\left(300 \mathrm{MHz}, \mathrm{CDCl}_{3}\right): \delta 7.37-7.29(\mathrm{~m}, 5 \mathrm{H}), 6.89(\mathrm{~d}, J=84.0 \mathrm{~Hz}, 1 \mathrm{H}), 3.73-$ $3.67(\mathrm{~m}, 2 \mathrm{H}), 2.86-2.81(\mathrm{~m}, 2 \mathrm{H}) \mathrm{ppm} ;{ }^{13} \mathrm{C}$ NMR $\left(75 \mathrm{MHz}, \mathrm{CDCl}_{3}\right): \delta 147.4$ $(\mathrm{d}, J=258.8 \mathrm{~Hz}), 136.1(\mathrm{~d}, J=9.0 \mathrm{~Hz}), 128.8,127.8,126.9(\mathrm{~d}, J=3.0 \mathrm{~Hz})$, $121.7(\mathrm{~d}, J=8.3 \mathrm{~Hz}), 60.8(\mathrm{~d}, J=3.0 \mathrm{~Hz}), 30.6(\mathrm{~d}, J=3.8 \mathrm{~Hz}) \mathrm{ppm} ;{ }^{19} \mathrm{~F}$ NMR $\left(282 \mathrm{MHz}, \mathrm{CDCl}_{3}\right): \delta$ -129.7 ppm; IR (neat): v 3363, 2963, 2886, 1659, 1118, 1044, 758, 696, 639, 594, $503 \mathrm{~cm}^{-1}$; HRMS (EI+) m/z: [M] ${ }^{+}$Calcd for $\mathrm{C}_{10} \mathrm{H}_{11} \mathrm{FO}$ 166.0794; Found 166.0798.

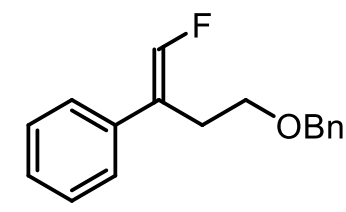

2v

(E)-(4-(benzyloxy)-1-fluorobut-1-en-2-yl)benzene 2v: obtained as a yellow oil ( $76 \%$ yield, $52 \mathrm{mg}$, d.r. = 96:4) after column chromatography on silica gel (pentane:Et ${ }_{2} \mathrm{O}$, from 100:0 to 95:5). Rf: 0.68 (pentane:Et ${ }_{2} \mathrm{O}$, 80:20); ${ }^{1} \mathrm{H}$ NMR (300 MHz, $\left.\mathrm{CDCl}_{3}\right): \delta 7.33-7.26(\mathrm{~m}, 10 \mathrm{H}), 6.84(\mathrm{~d}, J=87.0$ $\mathrm{Hz}, 1 \mathrm{H}), 4.47(\mathrm{~s}, 2 \mathrm{H}), 3.53(\mathrm{t}, J=7.5 \mathrm{~Hz}, 2 \mathrm{H}), 2.91-2.85(\mathrm{~m}, 2 \mathrm{H}) \mathrm{ppm} ;{ }^{13} \mathrm{C}$ NMR $\left(75 \mathrm{MHz}, \mathrm{CDCl}_{3}\right): \delta 147.0$ (d, $\left.J=258.8 \mathrm{~Hz}\right), 138.5,136.4$ (d, $J=9.0$ $\mathrm{Hz}), 128.7,128.4,127.7-127.6(\mathrm{~m}, 3 \mathrm{C}), 127.0(\mathrm{~d}, J=3.0 \mathrm{~Hz}), 122.1(\mathrm{~d}, J=8.3 \mathrm{~Hz}), 72.9,68.2(\mathrm{~d}$, $J=3.0 \mathrm{~Hz}$ ), $27.7(\mathrm{~d}, J=4.5 \mathrm{~Hz}) \mathrm{ppm} ;{ }^{19} \mathrm{~F} \mathrm{NMR}\left(282 \mathrm{MHz}, \mathrm{CDCl}_{3}\right): \delta-129.8 \mathrm{ppm} ; \mathrm{HRMS}(\mathrm{Cl}+) \mathrm{m} / \mathrm{z}$ : $[\mathrm{M}+\mathrm{H}]^{+}$Calcd for $\mathrm{C}_{17} \mathrm{H}_{18} \mathrm{FO} 257.1342$; Found: 257.1353 . 
$\overbrace{\text { OMOM }}^{\mathrm{F}}$

$2 w$ 90:10). Rf: 0.67 (pentane:Et $\left.{ }_{2} \mathrm{O}, 80: 20\right) ;{ }^{1} \mathbf{H}$ NMR (300 MHz, $\mathrm{CDCl}_{3}$ ): $\delta$ 7.43-7.26 (m, 5H), $6.84(\mathrm{~d}, J=84.0 \mathrm{~Hz}, 1 \mathrm{H}), 4.58(\mathrm{~s}, 2 \mathrm{H}), 3.57(\mathrm{t}, J=7.5$ $\mathrm{Hz}, 2 \mathrm{H}), 3.30(\mathrm{~s}, 3 \mathrm{H}), 2.88-2.82(\mathrm{~m}, 2 \mathrm{H}) \mathrm{ppm} ;{ }^{13} \mathrm{C} \mathrm{NMR}\left(75 \mathrm{MHz}, \mathrm{CDCl}_{3}\right)$ : $\delta 147.0(\mathrm{~d}, J=258.8 \mathrm{~Hz}), 136.2(\mathrm{~d}, J=9.0 \mathrm{~Hz}), 128.8,127.8,126.9(\mathrm{~d}, J=3.0 \mathrm{~Hz}), 122.0$ (d, $J=$ $9.0 \mathrm{~Hz}), 96.4,65.5(\mathrm{~d}, J=3.0 \mathrm{~Hz}), 55.2,27.7(\mathrm{~d}, J=3.8 \mathrm{~Hz}) \mathrm{ppm} ;{ }^{19} \mathrm{~F} \mathrm{NMR}\left(282 \mathrm{MHz}, \mathrm{CDCl}_{3}\right): \delta-$ 129.8 ppm; HRMS (El+) m/z: [M] ${ }^{+}$Calcd for $\mathrm{C}_{12} \mathrm{H}_{15} \mathrm{FO}_{2} 210.1056$; Found 210.1059.

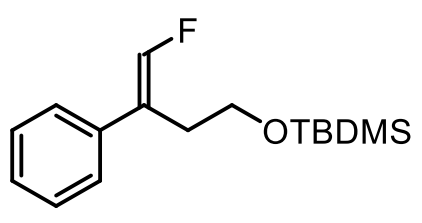

2x

(E)-tert-butyl((4-fluoro-3-phenylbut-3-en-1-yl)oxy)dimethylsilane 2x: obtained as pale yellow liquid (60\% yield, $42 \mathrm{mg}$, d.r. $=96: 4$ ) after column chromatography on silica gel (pentane: $\mathrm{Et}_{2} \mathrm{O}$, from 100:0 to 85:15). Rf: 0.42 (pentane:Et $\left.{ }_{2} \mathrm{O}, 80: 20\right) ;{ }^{1} \mathbf{H}$ NMR $(300 \mathrm{MHz}$, $\mathrm{CDCl}_{3}$ ): $\delta 7.43-7.26(\mathrm{~m}, 5 \mathrm{H}), 6.83(\mathrm{~d}, J=84.0 \mathrm{~Hz}, 1 \mathrm{H}), 3.66(\mathrm{t}, J=6.0$ $\mathrm{Hz}, 2 \mathrm{H}), 2.80-2.74(\mathrm{~m}, 2 \mathrm{H}), 0.86(\mathrm{~s}, 9 \mathrm{H}), 0.01(\mathrm{~s}, 6 \mathrm{H}) \mathrm{ppm} ;{ }^{13} \mathrm{C}$ NMR $\left(75 \mathrm{MHz}, \mathrm{CDCl}_{3}\right): \delta 147.0(\mathrm{~d}, J=258.8 \mathrm{~Hz}), 136.6(\mathrm{~d}, J=9.8 \mathrm{~Hz}), 128.7,127.6,126.9$ (d, $J=3.0$ $\mathrm{Hz}), 122.3(\mathrm{~d}, J=9.0 \mathrm{~Hz}), 61.4(\mathrm{~d}, J=3.0 \mathrm{~Hz}), 30.9(\mathrm{~d}, J=3.8 \mathrm{~Hz}), 26.0,18.4,-5.3 \mathrm{ppm} ;{ }^{19} \mathrm{~F}$ NMR $\left(282 \mathrm{MHz}, \mathrm{CDCl}_{3}\right): \delta-130.1 \mathrm{ppm}$; HRMS $(\mathrm{Cl}+) \mathrm{m} / \mathrm{z}$ : $[\mathrm{M}+\mathrm{H}]^{+}$Calcd for $\mathrm{C}_{16} \mathrm{H}_{26} \mathrm{FOSi} 281.1737$; Found 281.1738.<smiles>OCCC(=CF)c1ccccc1</smiles>

$2 u$

4-fluoro-3-phenylbut-3-en-1-ol 2u: obtained as a mixture of separable diastereomers $(80 \%$ yield, $44 \mathrm{mg}$, d.r. = 74:26) after column chromatography on silica gel ( $\mathrm{PE}: \mathrm{Et}_{2} \mathrm{O}$, from 90:10 to 50:50). Major diastereomer (E)-2u: already described page 23. Minor diastereomer (Z)$\mathbf{2 u}$ : obtained as a pale yellow liquid. Rf: 0.21 (pentane:Et $\left.{ }_{2} \mathrm{O}, 50: 50\right) ;{ }^{1} \mathbf{H}$ NMR (300 MHz, CDCl $): \delta 7.43-7.28(\mathrm{~m}, 5 \mathrm{H}), 6.71(\mathrm{~d}, J=84.0 \mathrm{~Hz}, 1 \mathrm{H}), 3.65-$ $3.61(\mathrm{~m}, 2 \mathrm{H}), 2.59-2.53(\mathrm{~m}, 2 \mathrm{H}) \mathrm{ppm} ;{ }^{13} \mathrm{C}$ NMR $\left(75 \mathrm{MHz}, \mathrm{CDCl}_{3}\right): \delta 145.4(\mathrm{~d}, J=263.0 \mathrm{~Hz}), 134.2$, 128.6, 128.3 (d, $J=4.5 \mathrm{~Hz}$ ), $127.9(\mathrm{~d}, J=0.8 \mathrm{~Hz}$ ), 119.1 (d, $J=1.5 \mathrm{~Hz}), 60.7$ (d, $J=3.8 \mathrm{~Hz}$ ), 33.8 $\left(\mathrm{d}, J=5.3 \mathrm{~Hz}\right.$ ) ppm; ${ }^{19} \mathrm{~F}$ NMR $\left(282 \mathrm{MHz}, \mathrm{CDCl}_{3}\right): \delta-128.7$ ppm; IR (neat): v 3374, 2925, 1665, $1445,1239,1162,1103,1045,771,696 \mathrm{~cm}^{-1}$.

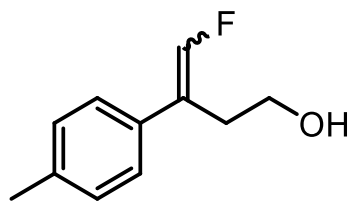

2y

4-fluoro-3-( $\boldsymbol{p}$-tolyl)but-3-en-1-ol $\mathbf{2 y}$ : obtained as a mixture of separable diastereomers (69\% yield, $38 \mathrm{mg}$, d.r. $=75: 25$ ) after column chromatography on silica gel (pentane:Et ${ }_{2} \mathrm{O}$, from 100:0 to 50:50). Major diastereomer $(E)-2 y$ : obtained as a colorless oil. Rf: 0.48 (pentane:Et $2 \mathrm{O}, 50: 50) ;{ }^{1} \mathrm{H}$ NMR $\left(300 \mathrm{MHz}, \mathrm{CDCl}_{3}\right): \delta$ 7.21-7.14 (m, 4H), $6.87(\mathrm{~d}, J=84.0 \mathrm{~Hz}, 1 \mathrm{H}), 3.72-3.66(\mathrm{~m}, 2 \mathrm{H}), 2.84-2.79(\mathrm{~m}, 2 \mathrm{H}), 2.35(\mathrm{~s}$, 3H) ppm; ${ }^{13} \mathrm{C} \mathrm{NMR}\left(75 \mathrm{MHz}, \mathrm{CDCl}_{3}\right): \delta 147.0(\mathrm{~d}, J=258.8 \mathrm{~Hz}), 137.7,133.1(\mathrm{~d}, J=9.0 \mathrm{~Hz}), 129.6$, $126.8(\mathrm{~d}, J=3.0 \mathrm{~Hz}), 121.6(\mathrm{~d}, J=9.0 \mathrm{~Hz}), 60.9(\mathrm{~d}, J=3.0 \mathrm{~Hz}), 30.6(\mathrm{~d}, J=3.8 \mathrm{~Hz}), 21.2 \mathrm{ppm} ;{ }^{19} \mathrm{~F}$ NMR (282 MHz, CDCl 3 ): $\delta$-130.7 ppm; IR (neat): v 3336, 2881, 1656, 1514, 1119, 1111, 1042, 
1020, 808, $506 \mathrm{~cm}^{-1}$; HRMS (Cl+) m/z: [M] Calcd for $\mathrm{C}_{11} \mathrm{H}_{13} \mathrm{FO}$ 180.0950; Found 180.0951. Minor diastereomer (Z)-2y: obtained as a pale yellow oil. Rf: 0.27 (pentane:Et $\left.{ }_{2} \mathrm{O}, 50: 50\right) ;{ }^{1} \mathbf{H}$ NMR (300 MHz, CDCl 3$): \delta$ 7.32-7.29 (m, 2H), 7.20-7.17 (m, 2H), $6.68(\mathrm{~d}, J=84.0 \mathrm{~Hz}, 1 \mathrm{H}), 3.65-$ $3.59(\mathrm{~m}, 2 \mathrm{H}), 2.57-2.51(\mathrm{~m}, 2 \mathrm{H}), 2.35(\mathrm{~s}, 3 \mathrm{H}) \mathrm{ppm} ;{ }^{13} \mathrm{C}$ NMR $\left(75 \mathrm{MHz}, \mathrm{CDCl}_{3}\right): \delta 145.2(\mathrm{~d}, J=$ $262.5 \mathrm{~Hz}), 137.7,131.1,129.3,128.2(\mathrm{~d}, J=4.5 \mathrm{~Hz}), 118.9(\mathrm{~d}, J=2.3 \mathrm{~Hz}), 60.7(\mathrm{~d}, J=3.8 \mathrm{~Hz})$, $33.7\left(\mathrm{~d}, J=5.3 \mathrm{~Hz}\right.$ ), $21.3 \mathrm{ppm} ;{ }^{19} \mathrm{~F}$ NMR $\left(282 \mathrm{MHz}, \mathrm{CDCl}_{3}\right): \delta-129.0 \mathrm{ppm}$; IR (neat): v 3337, 2924, 1660, 1515, 1100, 1044, 1020, 823, 497, $457 \mathrm{~cm}^{-1}$.

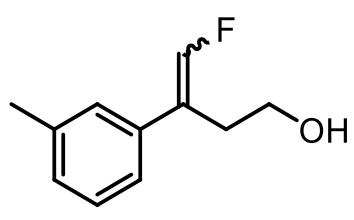

$2 z$

4-fluoro-3-( $m$-tolyl)but-3-en-1-ol 2z: obtained as a mixture of separable diastereomers (70\% yield, $39 \mathrm{mg}$, d.r. $=75: 25)$ after column chromatography on silica gel (pentane:Et ${ }_{2} \mathrm{O}$, from 100:0 to 50:50). Major diastereomer $(E)-2 z$ : obtained as a pale yellow oil. Rf: 0.59 (pentane: $\left.\mathrm{Et}_{2} \mathrm{O}, 50: 50\right) ;{ }^{1} \mathbf{H}$ NMR $\left(300 \mathrm{MHz}, \mathrm{CDCl}_{3}\right): \delta$ 7.24-7.21 $(\mathrm{m}, 1 \mathrm{H})$, 7.12-7.09 (m, 3H), $6.88(\mathrm{~d}, J=87.0 \mathrm{~Hz}, 1 \mathrm{H}), 3.73-3.66(\mathrm{~m}, 2 \mathrm{H}), 2.85-2.80$ (m, 2H), 2.35 (s, 3H) ppm; ${ }^{13} \mathrm{C}$ NMR (75 MHz, CDCl 3 ): $\delta 147.3$ (d, J = 258.8 Hz), 138.5, 136.0 (d, $J=9.0 \mathrm{~Hz}), 128.7,128.6,127.6(\mathrm{~d}, J=3.0 \mathrm{~Hz}), 124.0(\mathrm{~d}, J=3.0 \mathrm{~Hz}), 121.8(\mathrm{~d}, J=9 \mathrm{~Hz}), 60.9(\mathrm{~d}$, $J=2.3 \mathrm{~Hz}$ ), 30.7 (d, $\left.J=3.8 \mathrm{~Hz}), 21.6 \mathrm{ppm} ;{ }^{19} \mathrm{~F} \mathrm{NMR} \mathrm{(282} \mathrm{MHz}, \mathrm{CDCl}_{3}\right): \delta-130.1 \mathrm{ppm}$; IR (neat): v 3329, 2968, 1656, 1449, 1116, 1043, 782, $700 \mathrm{~cm}^{-1}$; HRMS (Cl+) m/z: [M]+Calcd for $\mathrm{C}_{11} \mathrm{H}_{13} \mathrm{FO}$ 180.0950; Found 180.0952. Minor diastereomer (Z)-2z: obtained as a pale yellow oil. Rf: 0.38 (pentane:Et $\left.{ }_{2} \mathrm{O}, 50: 50\right) ;{ }^{1} \mathrm{H}$ NMR $\left(300 \mathrm{MHz}, \mathrm{CDCl}_{3}\right): \delta 7.24-7.10(\mathrm{~m}, 4 \mathrm{H}), 6.69(\mathrm{~d}, J=84.0 \mathrm{~Hz}, 1 \mathrm{H})$, 3.65-3.59 (m, 2H), 2.57-2.51 (m, 2H), $2.36(\mathrm{~s}, 3 \mathrm{H}) \mathrm{ppm} ;{ }^{13} \mathrm{C} \mathrm{NMR}\left(75 \mathrm{MHz}, \mathrm{CDCl}_{3}\right): \delta 145.3$ (d, J $=262.5 \mathrm{~Hz}$ ), 138.2, 134.1, 129.0 (d, $J=4.5 \mathrm{~Hz}), 128.7,128.5,125.4$ (d, $J=3.8 \mathrm{~Hz}$ ), 119.2 (d, $J=$ $2.3 \mathrm{~Hz}$ ), 60.7 (d, $J=3.8 \mathrm{~Hz}), 33.8(\mathrm{~d}, J=5.3 \mathrm{~Hz}), 21.6 \mathrm{ppm} ;{ }^{19} \mathrm{~F} \mathrm{NMR}\left(282 \mathrm{MHz}, \mathrm{CDCl}_{3}\right): \delta-128.8$ ppm; IR (neat): v 3347, 2925, 1661, 1433, 1108, 1045, 1016, 788, $709 \mathrm{~cm}^{-1}$.

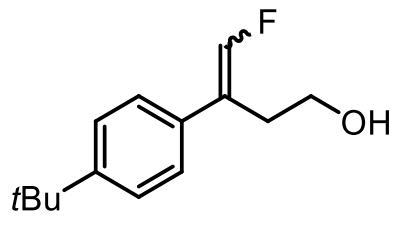

2aa

3-(4-(tert-butyl)phenyl)-4-fluorobut-3-en-1-ol 2aa: obtained as a mixture of separable diastereomers ( $96 \%$ yield, $58 \mathrm{mg}$, d.r. = 73:27) after column chromatography on silica gel (pentane: $\mathrm{Et}_{2} \mathrm{O}$, from 100:0 to 55:45). Major diastereomer (E)-2aa: obtained as a white amorphous solid. $\mathbf{R f :} 0.68$ (pentane: $\left.\mathrm{Et}_{2} \mathrm{O}, 40: 60\right) ;{ }^{1} \mathrm{H} \mathbf{~ N M R}(300 \mathrm{MHz}$, $\left.\mathrm{CDCl}_{3}\right): \delta$ 7.39-7.34 (m, 2H), 7.25-7.22 (m, 2H), $6.89(\mathrm{~d}, J=84.0 \mathrm{~Hz}$, $1 \mathrm{H}), 3.74-3.68(\mathrm{~m}, 2 \mathrm{H}), 2.85-2.80(\mathrm{~m}, 2 \mathrm{H}), 1.32(\mathrm{~s}, 9 \mathrm{H}) \mathrm{ppm} ;{ }^{13} \mathrm{C} \mathrm{NMR}\left(75 \mathrm{MHz}, \mathrm{CDCl}_{3}\right): \delta$ 150.9, $147.1(\mathrm{~d}, J=258.0 \mathrm{~Hz}$ ), $133.0(\mathrm{~d}, J=9.0 \mathrm{~Hz}), 126.5(\mathrm{~d}, J=3.0 \mathrm{~Hz}), 125.8,121.4(\mathrm{~d}, J=8.3 \mathrm{~Hz})$, $60.9(\mathrm{~d}, J=2.3 \mathrm{~Hz}), 34.7,31.4,30.6(\mathrm{~d}, J=3.0 \mathrm{~Hz}) \mathrm{ppm} ;{ }^{19} \mathrm{~F} \mathrm{NMR}\left(282 \mathrm{MHz}, \mathrm{CDCl}_{3}\right): \delta-130.6$ ppm; IR (neat): v 3310, 2960, 1657, 1124, 1107, 1052, 1038, 820, 561, $547 \mathrm{~cm}^{-1}$; HRMS (Cl+) $\mathrm{m} / \mathrm{z}$ : $[\mathrm{M}]^{+}$Calcd for $\mathrm{C}_{14} \mathrm{H}_{19} \mathrm{FO}$ 222.1420; Found 222.1413. Minor diastereomer (Z)-2aa: obtained as a yellow oil. Rf: 0.43 (pentane: $\left.\mathrm{Et}_{2} \mathrm{O}, 40: 60\right) ;{ }^{1} \mathbf{H} \mathbf{~ N M R}\left(300 \mathrm{MHz}, \mathrm{CDCl}_{3}\right): \delta$ 7.41-7.34 $(\mathrm{m}, 4 \mathrm{H}), 6.83-6.55(\mathrm{~m}, 1 \mathrm{H}), 3.66-3.60(\mathrm{~m}, 2 \mathrm{H}), 2.57-2.52(\mathrm{~m}, 2 \mathrm{H}), 1.33(\mathrm{~s}, 9 \mathrm{H}) \mathrm{ppm} ;{ }^{13} \mathrm{C}$ NMR (75 MHz, $\mathrm{CDCl}_{3}$ ): $\delta$ 150.8, 145.3 (d, J = 262.5 Hz), 131.0, 127.9 (d, J = 4.5 Hz), 125.5, 118.7 (d, J $=1.5 \mathrm{~Hz}), 60.7(\mathrm{~d}, J=3.8 \mathrm{~Hz}), 33.6(\mathrm{~d}, J=6.0 \mathrm{~Hz}), 31.4,29.9 \mathrm{ppm} ;{ }^{19} \mathrm{~F} \mathrm{NMR}\left(282 \mathrm{MHz}, \mathrm{CDCl}_{3}\right): \delta$ -128.8 ppm; IR (neat): v 3348, 2924, 1660, 1462, 1103, 1045, 1018, 839, $541 \mathrm{~cm}^{-1}$. 
<smiles>CN(C)c1ccc(C(=CF)CCO)cc1</smiles>

$2 a b$

3-(4-(dimethylamino)phenyl)-4-fluorobut-3-en-1-ol 2ab: obtained as a mixture of separable diastereomers (87\% yield, $51 \mathrm{mg}$, d.r. = 71:29) after column chromatography on silica gel (pentane: $\mathrm{Et}_{2} \mathrm{O}$, from 100:0 to 55:45). Major diastereomer (E)-2ab: obtained as an orange oil. Rf: 0.20 (cyclohexane:Et $\left.{ }_{2} \mathrm{O}, 50: 50\right) ;{ }^{1} \mathbf{H}$ NMR (300 MHz, $\mathrm{CDCl}_{3}$ ): $\delta$ 7.22-7.15 (m, 2H), $6.99(\mathrm{~s}, 0.5 \mathrm{H}), 6.73-6.67(\mathrm{~m}, 2.5 \mathrm{H}), 3.72-3.64(\mathrm{~m}$, $2 \mathrm{H}), 2.95(\mathrm{~s}, 6 \mathrm{H}), 2.82-2.77(\mathrm{~m}, 2 \mathrm{H}) \mathrm{ppm} ;{ }^{13} \mathrm{C} \mathrm{NMR}\left(75 \mathrm{MHz}, \mathrm{CDCl}_{3}\right): \delta 150.3,146.0$ (d, $J=256.5$ $\mathrm{Hz}), 127.6(\mathrm{~d}, J=3.0 \mathrm{~Hz}), 123.7(\mathrm{~d}, J=9.0 \mathrm{~Hz}), 121.3(\mathrm{~d}, J=8.3 \mathrm{~Hz}), 112.7,61.1(\mathrm{~d}, J=2.3 \mathrm{~Hz})$, 40.6, $30.6(\mathrm{~d}, J=3.0 \mathrm{~Hz})$ ppm; ${ }^{19} \mathrm{~F}$ NMR $\left(282 \mathrm{MHz}, \mathrm{CDCl}_{3}\right): \delta-133.3$ ppm; HRMS $(\mathrm{Cl}+) \mathrm{m} / \mathrm{z}:[\mathrm{M}]^{+}$ Calcd for $\mathrm{C}_{12} \mathrm{H}_{16} \mathrm{FNO}$ 209.1216; Found 209.1220. Minor diastereomer (Z)-2ab: obtained as an orange oil. Rf: 0.14 (cyclohexane: $\left.\mathrm{Et}_{2} \mathrm{O}, 50: 50\right) ;{ }^{1} \mathbf{H}$ NMR $\left(300 \mathrm{MHz}, \mathrm{CDCl}_{3}\right): \delta$ 7.36-7.32 (m, 2H), 6.74-6.68 (m, 2H), $6.64(\mathrm{~d}, J=84.0 \mathrm{~Hz}, 1 \mathrm{H}), 3.67-3.61(\mathrm{~m}, 2 \mathrm{H}), 2.97(\mathrm{~s}, 6 \mathrm{H}), 2.55-2.49(\mathrm{~m}, 2 \mathrm{H})$ ppm; ${ }^{13} \mathrm{C}$ NMR (75 MHz, $\left.\mathrm{CDCl}_{3}\right): \delta$ 150.0, $144.3(\mathrm{~d}, J=261.0 \mathrm{~Hz}), 129.1$ (d, J = 5.3 Hz), 121.6, $118.3,112.3,60.9(\mathrm{~d}, J=3.8 \mathrm{~Hz}), 40.5,33.5(\mathrm{~d}, J=6.8 \mathrm{~Hz}) \mathrm{ppm} ;{ }^{19} \mathrm{~F}$ NMR $\left(282 \mathrm{MHz}, \mathrm{CDCl}_{3}\right): \delta-$ 130.5 ppm; IR (neat): v 3347, 2883, 1610, 1521, 1353, 1107, 1043, 946, 813, 570, $523 \mathrm{~cm}^{-1}$.

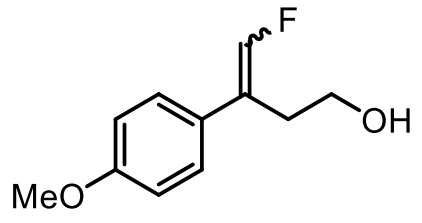

2ac

4-fluoro-3-(4-methoxyphenyl)but-3-en-1-ol 2ac: obtained as a mixture of separable diastereomers (72\% yield, $41 \mathrm{mg}$, d.r. = 72:28) after column chromatography on silica gel (pentane:Et ${ }_{2} \mathrm{O}$, from 100:0 to 50:50). Major diastereomer (E)-2ac: obtained as a colorless oil. Rf: 0.23 (cyclohexane:Et $\left.{ }_{2} \mathrm{O}, 50: 50\right) ;{ }^{1} \mathrm{H}$ NMR $(300 \mathrm{MHz}$, $\left.\mathrm{CDCl}_{3}\right): \delta 7.24-7.21(\mathrm{~m}, 2 \mathrm{H}), 6.90-6.86(\mathrm{~m}, 2 \mathrm{H}), 6.84(\mathrm{~d}, J=84.0 \mathrm{~Hz}$, $1 \mathrm{H}), 3.81(\mathrm{~s}, 3 \mathrm{H}), 3.72-3.66(\mathrm{~m}, 2 \mathrm{H}), 2.83-2.77(\mathrm{~m}, 2 \mathrm{H}) \mathrm{ppm} ;{ }^{13} \mathrm{C}$ NMR $\left(75 \mathrm{MHz}, \mathrm{CDCl}_{3}\right): \delta$ 159.4, 146.7 (d, $J=257.3 \mathrm{~Hz}$ ), 128.3 (d, $J=9.0 \mathrm{~Hz}$ ), 128.1 (d, $J=3.0 \mathrm{~Hz}$ ), 121.2 (d, $J=8.3 \mathrm{~Hz}$ ), 114.3, 60.9 (d, $J=2.3 \mathrm{~Hz}$ ), 55.4, 30.8 (d, $J=3.0 \mathrm{~Hz})$ ppm; ${ }^{19} \mathrm{~F} \mathrm{NMR}\left(282 \mathrm{MHz}, \mathrm{CDCl}_{3}\right): \delta-131.3$ ppm; IR (neat): v 3359, 2960, 1609, 1511, 1245, 1108, 1031, 817, 585, $525 \mathrm{~cm}^{-1}$; HRMS (Cl+) m/z: [M] ${ }^{+}$ Calcd for $\mathrm{C}_{11} \mathrm{H}_{13} \mathrm{FO}_{2}$ 196.0900; Found 196.0902. Minor diastereomer (Z)-2ac: obtained as a colorless oil. Rf: 0.16 (cyclohexane:Et $2 \mathrm{O}, 50: 50) ;{ }^{1} \mathbf{H}$ NMR (300 MHz, $\left.\mathrm{CDCl}_{3}\right): \delta$ 7.39-7.34 (m, $2 \mathrm{H}), 6.93-6.89(\mathrm{~m}, 2 \mathrm{H}), 6.67(\mathrm{~d}, J=84.0 \mathrm{~Hz}, 1 \mathrm{H}), 3.82(\mathrm{~s}, 3 \mathrm{H}), 3.64-3.60(\mathrm{~m}, 2 \mathrm{H}), 2.55-2.50(\mathrm{~m}$, 2H) ppm; ${ }^{13} \mathrm{C}$ NMR $\left(75 \mathrm{MHz}, \mathrm{CDCl}_{3}\right): \delta 159.1,144.9$ (d, $\left.J=261.8 \mathrm{~Hz}\right), 129.5$ (d, $\left.J=4.5 \mathrm{~Hz}\right), 126.3$, $118.4(\mathrm{~d}, J=1.5 \mathrm{~Hz}), 114.0,60.7(\mathrm{~d}, J=3.0 \mathrm{~Hz}), 55.4,33.7(\mathrm{~d}, J=6.0 \mathrm{~Hz}) \mathrm{ppm} ;{ }^{19} \mathrm{~F}$ NMR $(282$ $\mathrm{MHz}, \mathrm{CDCl}_{3}$ ): $\delta$-129.7 ppm; IR (neat): v 3354, 2938, 1608, 1512, 1248, 1180, 1097, 1031, 833, $806,506 \mathrm{~cm}^{-1}$.

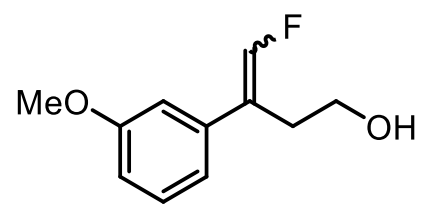

2ad

4-fluoro-3-(3-methoxyphenyl)but-3-en-1-ol 2ad: obtained as a mixture of separable diastereomers (73\% yield, $42 \mathrm{mg}$, d.r. $=82: 18$ ) after column chromatography on silica gel (pentane:Et ${ }_{2} \mathrm{O}$, from 100:0 to 60:40). Major diastereomer (E)-2ad: obtained as a colorless oil. Rf: 0.50 (pentane:Et $\left.{ }_{2} \mathrm{O}, 40: 60\right) ;{ }^{1} \mathbf{H}$ NMR $(300 \mathrm{MHz}$, $\left.\mathrm{CDCl}_{3}\right): \delta$ 7.29-7.23 (m, 1H), 6.91-6.83 (m, 3H), $6.90(\mathrm{~d}, J=87.0 \mathrm{~Hz}$, $1 \mathrm{H}), 3.82(\mathrm{~s}, 3 \mathrm{H}), 3.73-3.67(\mathrm{~m}, 2 \mathrm{H}), 2.84-2.79(\mathrm{~m}, 2 \mathrm{H}) \mathrm{ppm} ;{ }^{13} \mathrm{C} \mathrm{NMR}\left(75 \mathrm{MHz}, \mathrm{CDCl}_{3}\right): \delta$ 159.9, $147.5(\mathrm{~d}, J=259.5 \mathrm{~Hz}), 137.6(\mathrm{~d}, J=9.0 \mathrm{~Hz}), 129.9,121.7(\mathrm{~d}, J=8.3 \mathrm{~Hz}), 119.4(\mathrm{~d}, J=3.0 \mathrm{~Hz})$, 
113.0-112.9 (m, 2C), $60.9(\mathrm{~d}, J=3.0 \mathrm{~Hz}), 55.4,30.6(\mathrm{~d}, J=3.0 \mathrm{~Hz})$ ppm; ${ }^{19} \mathrm{~F}$ NMR $(282 \mathrm{MHz}$, $\mathrm{CDCl}_{3}$ ): $\delta$-129.6 ppm; IR (neat): v 3359, 2958, 1660, 1578, 1290, 1221, 1170, 1115, 1039, 778, $696 \mathrm{~cm}^{-1}$; HRMS $(\mathrm{Cl}+) \mathrm{m} / \mathrm{z}$ : [M] ${ }^{+}$Calcd for $\mathrm{C}_{11} \mathrm{H}_{13} \mathrm{FO}_{2}$ 196.0900; Found 196.0893. Minor diastereomer (Z)-2ad: obtained as a pale yellow oil. Rf: 0.31 (pentane:Et $\left.{ }_{2} \mathrm{O}, 40: 60\right) ;{ }^{\mathbf{H}} \mathbf{~ N M R}$ $\left(300 \mathrm{MHz}, \mathrm{CDCl}_{3}\right): \delta$ 7.32-7.27 $(\mathrm{m}, 1 \mathrm{H}), 7.00-6.95(\mathrm{~m}, 2 \mathrm{H}), 6.87-6.83(\mathrm{~m}, 1.5 \mathrm{H}), 6.56-6.55(\mathrm{~m}$, $0.5 \mathrm{H}), 3.82(\mathrm{~s}, 3 \mathrm{H}), 3.65-3.61(\mathrm{~m}, 2 \mathrm{H}), 2.57-2.51(\mathrm{~m}, 2 \mathrm{H}) \mathrm{ppm} ;{ }^{13} \mathrm{C} \mathrm{NMR}\left(75 \mathrm{MHz}, \mathrm{CDCl}_{3}\right): \delta$ 159.7, 145.5 (d, $J=263.3 \mathrm{~Hz}), 135.5,129.6,120.7(\mathrm{~d}, J=3.8 \mathrm{~Hz}), 119.0,114.3(\mathrm{~d}, J=4.5 \mathrm{~Hz})$, 113.2, $60.7\left(\mathrm{~d}, J=3.0 \mathrm{~Hz}\right.$ ), 55.4, 33.9 ppm; $\left.{ }^{19} \mathrm{~F} \mathrm{NMR} \mathrm{(282} \mathrm{MHz,} \mathrm{CDCl}_{3}\right): \delta-127.9$ ppm; IR (neat): v $3359,2941,1577,1261,1215,1107,1043,782,699 \mathrm{~cm}^{-1}$.

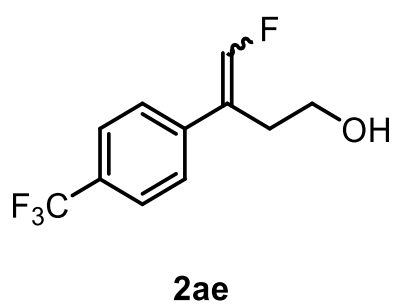

4-fluoro-3-(4-(trifluoromethyl)phenyl)but-3-en-1-ol 2ae: obtained as a mixture of separable diastereomers $(81 \%$ yield, $32 \mathrm{mg}$, d.r. = 69:31) after column chromatography on silica gel (pentane:Et ${ }_{2} \mathrm{O}$, from 100:0 to 50:50). Major diastereomer (E)-2ae: obtained as a pale yellow oil. Rf: 0.35 (pentane:Et $\left.{ }_{2} \mathrm{O}, 50: 50\right) ;{ }^{1} \mathbf{H}$ NMR $(300 \mathrm{MHz}$, $\left.\mathrm{CDCl}_{3}\right): \delta$ 7.62-7.59 (m, 2H), 7.45-7.42 (m, 2H), $6.93(\mathrm{~d}, J=84.0 \mathrm{~Hz}$, 1H), 3.75-3.68 (m, 2H), 2.87-2.82 (m, 2H) ppm; ${ }^{13} \mathrm{C} \mathrm{NMR} \mathrm{(75} \mathrm{MHz,} \mathrm{CDCl} 3$ ): $\delta 148.3(\mathrm{~d}, J=261.8$ $\mathrm{Hz}), 140.0(\mathrm{~d}, J=8.3 \mathrm{~Hz}), 130.0(\mathrm{q}, J=32.8 \mathrm{~Hz}), 127.2(\mathrm{~d}, J=3.0 \mathrm{~Hz}), 125.8(\mathrm{q}, J=3.8 \mathrm{~Hz}), 124.2$ $(\mathrm{q}, J=270.0 \mathrm{~Hz}), 121.2(\mathrm{~d}, J=9.8 \mathrm{~Hz}), 60.7(\mathrm{~d}, J=2.3 \mathrm{~Hz}), 30.4(\mathrm{~d}, J=2.3 \mathrm{~Hz}) \mathrm{ppm} ;{ }^{19} \mathrm{~F} \mathrm{NMR}$ (282 MHz, $\mathrm{CDCl}_{3}$ ): $\delta$-63.1 (3F), -126.8 ppm; IR (neat): v 3348, 2936, 1660, 1324, 1165, 1119, 1111, 1070, 1045, 1016, $826 \mathrm{~cm}^{-1}$; HRMS (Cl+) m/z: [M] ${ }^{+}$Calcd for $\mathrm{C}_{11} \mathrm{H}_{10} \mathrm{~F}_{4} \mathrm{O}$ 234.0668; Found 234.0662. Minor diastereomer (Z)-2ae: obtained as a yellow oil. Rf: 0.13 (pentane:Et ${ }_{2} \mathrm{O}$, 50:50); ${ }^{1} \mathrm{H}$ NMR (300 MHz, $\left.\mathrm{CDCl}_{3}\right): \delta 7.65-7.62(\mathrm{~m}, 2 \mathrm{H}), 7.54-7.51(\mathrm{~m}, 2 \mathrm{H}), 6.76(\mathrm{~d}, J=84.0 \mathrm{~Hz}$, $1 \mathrm{H})$, 3.66-3.60 (m, 2H), 2.61-2.55 (m, 2H); $\left.{ }^{13} \mathrm{C} \mathrm{NMR} \mathrm{(75} \mathrm{MHz,} \mathrm{CDCl} 3\right): \delta 146.4(\mathrm{~d}, J=265.5 \mathrm{~Hz})$, $138.0(\mathrm{~d}, J=0.8 \mathrm{~Hz}$ ), $130.3(\mathrm{q}, J=33.0 \mathrm{~Hz}), 128.7$ (d, $J=4.5 \mathrm{~Hz}), 125.5(\mathrm{q}, J=3.8 \mathrm{~Hz}$ ), 124.2 (q, $J=270.0 \mathrm{~Hz}$ ), $118.3(\mathrm{~d}, J=1.5 \mathrm{~Hz}), 60.5(\mathrm{~d}, J=3.0 \mathrm{~Hz}), 30.5 \mathrm{ppm} ;{ }^{19} \mathrm{~F} \mathrm{NMR}\left(282 \mathrm{MHz}, \mathrm{CDCl}_{3}\right): \delta$ -63.2 (3F), -126.6 ppm; IR (neat): v 3359, 2958, 1664, 1322, 1165, 1121, 1103, 1064, 1017, 847 $\mathrm{cm}^{-1}$.

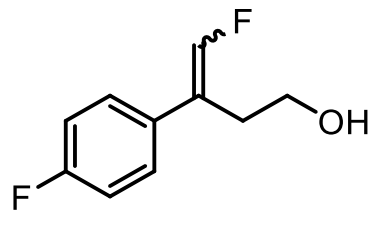

2af

4-fluoro-3-(4-fluorophenyl)but-3-en-1-ol 2af: obtained as a mixture of separable diastereomers (71\% yield, $25 \mathrm{mg}$, d.r. = 74:26) after column chromatography on silica gel (pentane: $\mathrm{Et}_{2} \mathrm{O}$, from 100:0 to 50:50). Major diastereomer (E)-2af: obtained as a pale yellow oil. $\mathbf{R f :}$ 0.45 (pentane: $\left.\mathrm{Et}_{2} \mathrm{O}, 50: 50\right) ;{ }^{1} \mathrm{H}$ NMR $\left(300 \mathrm{MHz}, \mathrm{CDCl}_{3}\right): \delta 7.30-7.24(\mathrm{~m}$, $2 \mathrm{H}), 7.07-7.01(\mathrm{~m}, 2 \mathrm{H}), 6.83(\mathrm{~d}, J=84.0 \mathrm{~Hz}, 1 \mathrm{H}), 3.72-3.66(\mathrm{~m}, 2 \mathrm{H})$, 2.83-2.77 (m, 2H) ppm; ${ }^{13} \mathrm{C} \mathrm{NMR} \mathrm{(75} \mathrm{MHz,} \mathrm{CDCl} 3$ ): $\delta 162.6(\mathrm{dd}, J=245.3 \& 0.8 \mathrm{~Hz}), 147.2$ (dd, $J=259.1 \& 1.1 \mathrm{~Hz}), 128.7(\mathrm{~d}, J=8.3 \mathrm{~Hz}), 128.6(\mathrm{~d}, J=8.3 \mathrm{~Hz}), 121.1(\mathrm{~d}, J=9.8 \mathrm{~Hz}), 115.8(\mathrm{~d}, J=$ $21.0 \mathrm{~Hz}$ ), 60.8 (d, $J=2.3 \mathrm{~Hz}), 30.9$ (d, $J=3.8 \mathrm{~Hz})$ ppm; ${ }^{19} \mathbf{F}$ NMR $\left(282 \mathrm{MHz}, \mathrm{CDCl}_{3}\right): \delta-115.1$, 129.4 ppm; IR (neat): v 3339, 2963, 2881, 1659, 1603, 1509, 1224, 1161, 1118, 1043, 821, 575, $517 \mathrm{~cm}^{-1}$; HRMS (AP-) m/z: [M - H] Calcd for $\mathrm{C}_{10} \mathrm{H}_{9} \mathrm{~F}_{2} \mathrm{O}$ 183.0621; Found 183.0615. Minor diastereomer (Z)-2af: obtained as a pale yellow oil. $\mathbf{R f}$ : 0.27 (pentane:Et $\left.{ }_{2} \mathrm{O}, 50: 50\right) ;{ }^{\mathbf{H}} \mathbf{~ N M R}$ $\left(300 \mathrm{MHz}, \mathrm{CDCl}_{3}\right): \delta$ 7.42-7.36 (m, 2H), 7.10-7.03 (m, 2H), $6.70(\mathrm{~d}, J=84.0 \mathrm{~Hz}, 1 \mathrm{H}), 3.65-3.59$ 
(m, 2H), 2.56-2.51 (m, 2H) ppm; ${ }^{13} \mathrm{C}$ NMR $\left(75 \mathrm{MHz}, \mathrm{CDCl}_{3}\right): \delta 162.2(\mathrm{dd}, J=245.6 \& 1.1 \mathrm{~Hz}$ ), $145.4(\mathrm{dd}, J=262.9$ \& $1.1 \mathrm{~Hz}), 130.1(\mathrm{~d}, J=4.5 \mathrm{~Hz}), 130.0(\mathrm{~d}, J=4.5 \mathrm{~Hz}), 118.2(\mathrm{~d}, J=1.5 \mathrm{~Hz})$, $115.6(\mathrm{~d}, J=21.0 \mathrm{~Hz}), 60.6(\mathrm{~d}, J=3.0 \mathrm{~Hz}), 33.8(\mathrm{~d}, J=5.3 \mathrm{~Hz}) \mathrm{ppm} ;{ }^{19} \mathrm{~F}$ NMR $\left(282 \mathrm{MHz}, \mathrm{CDCl}_{3}\right)$ : $\delta-114.5,-128.8$ ppm; IR (neat): v 3376, 2931, 2871, 1663, 1604, 1510, 1232, 1162, 1095, 1046, $839 \mathrm{~cm}^{-1}$.<smiles>OCCC(=CF)c1ccc(Br)cc1</smiles>

2ag

3-(4-bromophenyl)-4-fluorobut-3-en-1-ol 2ag: obtained as a mixture of separable diastereomers (87\% yield, $54 \mathrm{mg}$, d.r. $=66: 34$ ) after column chromatography on silica gel (pentane: $\mathrm{Et}_{2} \mathrm{O}$, from 100:0 to 50:50). Major diastereomer (E)-2ag: obtained as a pale yellow oil. Rf: 0.35 (pentane:Et $\left.{ }_{2} \mathrm{O}, 50: 50\right) ;{ }^{1} \mathbf{H}$ NMR $\left(300 \mathrm{MHz}_{\mathrm{CDCl}}\right.$ ): $\delta$ 7.51-7.45 (m, $2 \mathrm{H}), 7.23-7.16(\mathrm{~m}, 2 \mathrm{H}), 6.87(\mathrm{~d}, J=84.0 \mathrm{~Hz}, 1 \mathrm{H}), 3.72-3.63(\mathrm{~m}, 2 \mathrm{H})$, 2.83-2.77 (m, 2H) ppm; $\left.{ }^{13} \mathrm{C} \mathrm{NMR} \mathrm{(75} \mathrm{MHz,} \mathrm{CDCl}\right): \delta 147.5(\mathrm{~d}, J=261.0 \mathrm{~Hz}), 135.1$ (d, $J=9.0$ $\mathrm{Hz}), 132.0,130.0,128.5(\mathrm{~d}, J=3.0 \mathrm{~Hz}), 121.1(\mathrm{~d}, J=9.8 \mathrm{~Hz}), 60.7(\mathrm{~d}, J=3.0 \mathrm{~Hz}), 30.5(\mathrm{~d}, J=3.0$ $\mathrm{Hz}$ ) ppm; $\left.{ }^{19} \mathrm{~F} \mathrm{NMR} \mathrm{(282} \mathrm{MHz,} \mathrm{CDCl}_{3}\right): \delta-128.5$ ppm; IR (neat): v 3321, 2925, 1656, 1488, 1240, 1122, 1074, 1043, 1008, 814, $504 \mathrm{~cm}^{-1}$; HRMS (Cl+) m/z: [M] ${ }^{+}$Calcd for $\mathrm{C}_{10} \mathrm{H}_{10} \mathrm{BrFO} 243.9899$; Found 243.9901. Minor diastereomer (Z)-2ag: obtained as a colorless oil. Rf: 0.15 (pentane:Et $2 \mathrm{O}, 50: 50) ;{ }^{1} \mathrm{H}$ NMR (300 MHz, $\left.\mathrm{CDCl}_{3}\right): \delta$ 7.52-7.48 (m, 2H), 7.31-7.27 (m, 2H), 6.85$6.57(\mathrm{~m}, 1 \mathrm{H}), 3.65-3.59(\mathrm{~m}, 2 \mathrm{H}), 2.56-2.50(\mathrm{~m}, 2 \mathrm{H}) \mathrm{ppm} ;{ }^{13} \mathrm{C} \mathrm{NMR}\left(75 \mathrm{MHz}, \mathrm{CDCl}_{3}\right): \delta 145.8(\mathrm{~d}$, $J=264.0 \mathrm{~Hz}$ ), 133.1, 131.8, 130.0 (d, J=4.5 Hz), 125.7, 118.2 (d, J=2.3 Hz), 60.5 (d, J = 3.8 Hz), $33.5(\mathrm{~d}, J=5.3 \mathrm{~Hz}) \mathrm{ppm} ;{ }^{19} \mathrm{~F}$ NMR $\left(282 \mathrm{MHz}, \mathrm{CDCl}_{3}\right): \delta-127.4$ ppm; IR (neat): v 3354, 2951, $1661,1489,1099,1085,1045,1009,830,494 \mathrm{~cm}^{-1}$.<smiles>OCCC(=CF)c1cccc(Cl)c1</smiles>

2ah

3-(3-chlorophenyl)-4-fluorobut-3-en-1-ol 2ah: obtained as a mixture of separable diastereomers (81\% yield, $47 \mathrm{mg}$, d.r. $=74: 26)$ after column chromatography on silica gel (pentane:Et ${ }_{2} \mathrm{O}$, from 100:0 to 50:50). Major diastereomer (E)-2ah: obtained as a colorless oil. Rf: 0.43 (pentane: $\left.\mathrm{Et}_{2} \mathrm{O}, 50: 50\right) ;{ }^{1} \mathrm{H}$ NMR $\left(300 \mathrm{MHz} \mathrm{CDCl}_{3}\right): \delta 7.31-7.17(\mathrm{~m}$, $4 \mathrm{H}), 6.89(\mathrm{~d}, J=84.0 \mathrm{~Hz}, 1 \mathrm{H}), 3.72-3.67(\mathrm{~m}, 2 \mathrm{H}), 2.83-2.77(\mathrm{~m}, 2 \mathrm{H})$ ppm; ${ }^{13} \mathrm{C}$ NMR $\left(75 \mathrm{MHz}, \mathrm{CDCl}_{3}\right): \delta 147.9(\mathrm{~d}, J=261.0 \mathrm{~Hz}), 138.1(\mathrm{~d}, J=9.0 \mathrm{~Hz}), 134.8,130.1$, 127.9, $127.0(\mathrm{~d}, J=3.0 \mathrm{~Hz}), 125.1(\mathrm{~d}, J=4.0 \mathrm{~Hz}), 121.0(\mathrm{~d}, J=9.8 \mathrm{~Hz}), 60.7(\mathrm{~d}, J=2.3 \mathrm{~Hz}), 30.5$ (d, $J=3.8 \mathrm{~Hz}$ ) ppm; ${ }^{19} \mathrm{~F}$ NMR $\left(282 \mathrm{MHz}, \mathrm{CDCl}_{3}\right): \delta-128.0$ ppm; IR (neat): v 3332, 2887, 1655, 1410, 1124, 1044, 780, $691 \mathrm{~cm}^{-1}$; HRMS (Cl+) m/z: [M] ${ }^{+}$Calcd for $\mathrm{C}_{10} \mathrm{H}_{10} \mathrm{ClFO}$ 200.0404; Found 200.0410. Minor diastereomer (Z)-2ah: obtained as a pale yellow oil. Rf: 0.22 (pentane:Et ${ }_{2} \mathrm{O}$, 50:50); ${ }^{1} \mathbf{H}$ NMR (300 MHz, $\left.\mathrm{CDCl}_{3}\right): \delta$ 7.41-7.40 (m, 1H), 7.32-7.27 (m, 3H), 6.85-6.57 (m, $\left.1 \mathrm{H}\right)$, 3.65-3.60 (m, 2H), 2.55-2.51 (m, 2H) ppm; $\left.{ }^{13} \mathrm{C} \mathrm{NMR} \mathrm{(75} \mathrm{MHz,} \mathrm{CDCl}_{3}\right): \delta 146.0(\mathrm{~d}, J=264.8 \mathrm{~Hz})$, 136.0, 134.5, 129.8, 128.4 (d, $J=4.5 \mathrm{~Hz}), 128.0,126.5$ (d, J=4.5 Hz), 118.1 (d, J=1.5 Hz), 60.5 (d, $J=3.8 \mathrm{~Hz}$ ), 33.5 (d, $J=5.3 \mathrm{~Hz}$ ) ppm; ${ }^{19} \mathrm{~F}$ NMR (282 MHz, $\left.\mathrm{CDCl}_{3}\right): \delta-127.0$ ppm; IR (neat): v $3347,2927,1662,1476,1108,1046,1016,785,700,675 \mathrm{~cm}^{-1}$. 


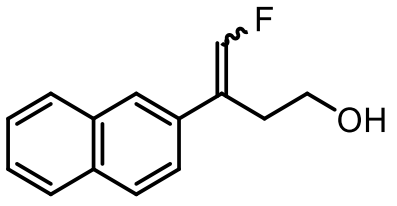

2ai

4-fluoro-3-(naphthalen-2-yl)but-3-en-1-ol 2ai: obtained as a mixture of separable diastereomers (63\% yield, $37 \mathrm{mg}$, d.r. $=76: 24$ ) after column chromatography on silica gel (pentane:Et ${ }_{2} \mathrm{O}$, from 100:0 to 50:50). Major diastereomer (E)-2ai: obtained as a white amorphous solid. $\mathbf{R f :} 0.58$ (pentane: $\left.\mathrm{Et}_{2} \mathrm{O}, 50: 50\right) ;{ }^{1} \mathbf{H} \mathbf{~ N M R}(300 \mathrm{MHz}$, $\left.\mathrm{CDCl}_{3}\right): \delta$ 7.84-7.75 (m, 4H), 7.52-7.42 (m, 3H), $7.02(\mathrm{~d}, J=84.0 \mathrm{~Hz}$, $1 \mathrm{H})$, 3.78-3.72 (m, 2H), 2.97-2.91 (m, 2H) ppm; ${ }^{13} \mathrm{C} \mathrm{NMR} \mathrm{(75} \mathrm{MHz,} \mathrm{CDCl} 3$ ): $\delta 147.7$ (d, $J=259.5$ $\mathrm{Hz}), 133.5,133.4$ (d, $J=9.0 \mathrm{~Hz}), 132.9,128.6,128.0,127.7,126.6,126.2,125.7$ (d, $J=3.8 \mathrm{~Hz})$, $124.8(\mathrm{~d}, J=2.3 \mathrm{~Hz}), 121.8(\mathrm{~d}, J=9.0 \mathrm{~Hz}), 60.9(\mathrm{~d}, J=3.0 \mathrm{~Hz}), 30.5(\mathrm{~d}, J=3.0 \mathrm{~Hz}) \mathrm{ppm} ;{ }^{19} \mathrm{~F}$ NMR (282 MHz, CDCl $)$ ): $\delta$-128.9 ppm; IR (neat): v 3305, 2960, 1653, 1111, 1043, 1020, 813, 748, $478 \mathrm{~cm}^{-1}$; HRMS $(\mathrm{Cl}+) \mathrm{m} / \mathrm{z}$ : $[\mathrm{M}]^{+}$Calcd for $\mathrm{C}_{14} \mathrm{H}_{13} \mathrm{FO}$ 216.0950; Found 216.0953. Minor diastereomer (Z)-2ai: obtained as a pale yellow oil. Rf: 0.22 (pentane:Et ${ }_{2} \mathrm{O}, 50: 50$ ); ${ }^{\mathbf{1}} \mathbf{~ N M R}$ (300 MHz, $\left.\mathrm{CDCl}_{3}\right): \delta$ 7.86-7.81 (m, 4H), 7.56-7.47 (m, 3H), $6.79(\mathrm{~d}, J=84.0 \mathrm{~Hz}, 1 \mathrm{H}), 3.69-3.64$ (m, 2H), 2.70-2.64 (m, 2H) ppm; ${ }^{13} \mathrm{C}$ NMR (75 MHz, CDCl $): \delta 145.7(\mathrm{~d}, J=263.3 \mathrm{~Hz}), 135.9$, 133.4, 132.9, 131.6, 128.2 (d, $J=6.0 \mathrm{~Hz}), 127.7,127.4(\mathrm{~d}, J=3.8 \mathrm{~Hz}), 126.4,126.3$ (d, $J=4.5$ $\mathrm{Hz}), 125.7,119.1(\mathrm{~d}, J=2.3 \mathrm{~Hz}), 60.7(\mathrm{~d}, J=3.8 \mathrm{~Hz}), 33.8(\mathrm{~d}, J=6.0 \mathrm{~Hz}) \mathrm{ppm} ;{ }^{19} \mathrm{~F} \mathrm{NMR}(282 \mathrm{MHz}$, $\mathrm{CDCl}_{3}$ ): $\delta$-128.2 ppm; IR (neat): v 3338, 2926, 1660, 1103, 1045, 1015, 859, 818, 748, $475 \mathrm{~cm}^{-}$ 1 .

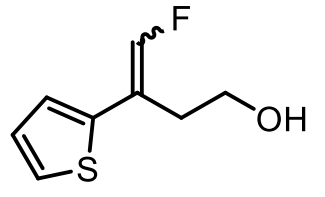

2aj

4-fluoro-3-(thiophen-2-yl)but-3-en-1-ol 2aj: obtained as a mixture of separable diastereomers (73\% yield, $40 \mathrm{mg}$, d.r. $=68: 32)$ after column chromatography on silica gel (pentane: $\mathrm{Et}_{2} \mathrm{O}$, from 100:0 to 50:50). Major diastereomer (E)-2aj: obtained as a pale yellow oil. $\mathbf{R f :} 0.38$ (pentane: $\left.\mathrm{Et}_{2} \mathrm{O}, 50: 50\right) ;{ }^{1} \mathrm{H}$ NMR $\left(300 \mathrm{MHz} \mathrm{CDCl}_{3}\right): \delta 7.25(\mathrm{~s}, 0.5 \mathrm{H}), 7.20$ $7.18(\mathrm{~m}, 1 \mathrm{H}), 7.03-6.97(\mathrm{~m}, 2.5 \mathrm{H}), 3.82-3.77(\mathrm{~m}, 2 \mathrm{H}), 2.84-2.79(\mathrm{~m}, 2 \mathrm{H})$ ppm; ${ }^{13} \mathrm{C}$ NMR $\left(75 \mathrm{MHz}, \mathrm{CDCl}_{3}\right): \delta 146.9(\mathrm{~d}, J=261.0 \mathrm{~Hz}), 138.8(\mathrm{~d}, J=9.0 \mathrm{~Hz}), 127.6,124.4-$ $124.3(\mathrm{~m}, 2 \mathrm{C}), 116.5(\mathrm{~d}, J=12.8 \mathrm{~Hz}), 61.0(\mathrm{~d}, J=2.3 \mathrm{~Hz}), 31.4(\mathrm{~d}, J=3.0 \mathrm{~Hz}) \mathrm{ppm} ;{ }^{19} \mathrm{~F} \mathrm{NMR}(282$ $\mathrm{MHz}, \mathrm{CDCl}_{3}$ ): $\delta-130.1$ ppm; IR (neat): v 3332, 3082, 2963, 2887, 1650, 1113, 1041, 809, 693 $\mathrm{cm}^{-1}$; HRMS $(\mathrm{Cl}+) \mathrm{m} / \mathrm{z}$ : [M] ${ }^{+}$Calcd for $\mathrm{C}_{8} \mathrm{H}_{9} \mathrm{FOS}$ 172.0358; Found 172.0353. Minor diastereomer (Z)-2aj: obtained as a pale yellow oil. Rf: 0.20 (pentane:Et $2 \mathrm{O}, 50: 50) ;{ }^{1} \mathbf{H ~ N M R}\left(300 \mathrm{MHz}_{\mathrm{N}} \mathrm{CDCl}_{3}\right.$ ): $\delta$ 7.36-7.34 (m, $1 \mathrm{H}), 7.20-7.19(\mathrm{~m}, 1 \mathrm{H}), 7.07-7.04(\mathrm{~m}, 1 \mathrm{H}), 6.72(\mathrm{~d}, J=81.0 \mathrm{~Hz}, 1 \mathrm{H}), 3.83-3.79$ (m, 2H), 2.63-2.58 (m, 2H) ppm; ${ }^{13} \mathrm{C} \mathrm{NMR}\left(75 \mathrm{MHz}, \mathrm{CDCl}_{3}\right): \delta 145.2(\mathrm{~d}, J=267.8 \mathrm{~Hz}$ ), 136.4 (d, $J=4.5 \mathrm{~Hz}), 126.8,126.1(\mathrm{~d}, J=9.0 \mathrm{~Hz}), 125.7(\mathrm{~d}, J=5.3 \mathrm{~Hz}), 113.0(\mathrm{~d}, J=2.3 \mathrm{~Hz}), 61.0(\mathrm{~d}, J=3.8$ $\mathrm{Hz}$ ), 33.4 (d, $J=6.8 \mathrm{~Hz}$ ) ppm; ${ }^{19} \mathrm{~F} \mathrm{NMR}\left(282 \mathrm{MHz}, \mathrm{CDCl}_{3}\right): \delta-120.5$ ppm; IR (neat): v 3343, 2952, $2892,1650,1234,1108,1045,697 \mathrm{~cm}^{-1}$.<smiles>CC(CO)C(=CF)c1ccccc1</smiles>

2ak 4-fluoro-2-methyl-3-phenylbut-3-en-1-ol 2ak: obtained as a mixture of separable diastereomers (72\% yield, $40 \mathrm{mg}$, d.r. $=50: 50$ ) after column chromatography on silica gel (pentane:Et ${ }_{2} \mathrm{O}$, from 100:0 to 50:50). Major diastereomer (E)-2ak: obtained as a pale yellow oil. Rf: 0.45 (pentane: $\left.\mathrm{Et}_{2} \mathrm{O}, 50: 50\right) ;{ }^{1} \mathrm{H}$ NMR (300 MHz, $\left.\mathrm{CDCl}_{3}\right): \delta 7.35-7.23(\mathrm{~m}, 5 \mathrm{H})$, $6.64(\mathrm{~d}, J=84.0 \mathrm{~Hz}, 1 \mathrm{H}), 3.74-3.61(\mathrm{~m}, 2 \mathrm{H}), 3.18-3.06(\mathrm{~m}, 1 \mathrm{H}), 1.13(\mathrm{~d}, J=$ 
$9.0 \mathrm{~Hz}, 3 \mathrm{H}) \mathrm{ppm} ;{ }^{13} \mathrm{C}$ NMR $\left(75 \mathrm{MHz}, \mathrm{CDCl}_{3}\right): \delta 147.2(\mathrm{~d}, J=261.0 \mathrm{~Hz}), 135.8(\mathrm{~d}, J=10.5 \mathrm{~Hz})$, $129.0(\mathrm{~d}, J=3.0 \mathrm{~Hz}$ ), $128.5,127.8,127.0$ (d, $J=6.0 \mathrm{~Hz}), 65.8$ (d, $J=4.0 \mathrm{~Hz}$ ), 37.2, 15.5 (d, J = 4.0 $\mathrm{Hz}$ ) ppm; ${ }^{19} \mathrm{~F}$ NMR $\left(282 \mathrm{MHz}, \mathrm{CDCl}_{3}\right): \delta$-129.2 ppm; IR (neat): v 3344, 2931, 1656, 1444, 1107, 1063, 1027, 761, 699, 655, $646 \mathrm{~cm}^{-1}$; HRMS (Cl+) m/z: [M] ${ }^{+}$Calcd for $\mathrm{C}_{11} \mathrm{H}_{13} \mathrm{FO}$ 180.0950; Found 180.0946. Minor diastereomer (Z)-2ak: obtained as a yellow oil. Rf: 0.25 (pentane:Et ${ }_{2} \mathrm{O}$, 50:50); ${ }^{1} \mathrm{H}$ NMR $\left(300 \mathrm{MHz}, \mathrm{CDCl}_{3}\right): \delta 7.41-7.26(\mathrm{~m}, 5 \mathrm{H}), 6.66(\mathrm{~d}, J=84.0 \mathrm{~Hz}, 1 \mathrm{H}), 3.59-3.46(\mathrm{~m}$, $2 \mathrm{H}), 2.74-2.63(\mathrm{~m}, 1 \mathrm{H}), 1.13(\mathrm{~d}, J=6.0 \mathrm{~Hz}, 3 \mathrm{H}) \mathrm{ppm} ;{ }^{13} \mathrm{C} \mathrm{NMR}\left(75 \mathrm{MHz}, \mathrm{CDCl}_{3}\right): \delta 145.7(\mathrm{~d}, J=$ $257.3 \mathrm{~Hz}$ ), 134.4 (d, J = 3.0 Hz), 128.9 (d, J = 2.3 Hz), 128.4, 127.7, 125.4 (d, J = 3.0 Hz), 65.7 (d, $J=3.0 \mathrm{~Hz}$ ), $38.5(\mathrm{~d}, J=4.5 \mathrm{~Hz}), 16.0(\mathrm{~d}, J=2.3 \mathrm{~Hz})$ ppm; ${ }^{19} \mathrm{~F} \mathrm{NMR}\left(282 \mathrm{MHz}, \mathrm{CDCl}_{3}\right): \delta-132.9$ ppm; IR (neat): v 3350, 2931, 1664, 1495, 1443, 1027, 771, 698, $664 \mathrm{~cm}^{-1}$. 


\section{References}

[1] For the synthesis of $\mathbf{1 b}$ and $\mathbf{1 n}$ : K. M. Koshy, D. Roy, T. T. Tidwell, J. Am. Chem. Soc. 1979, 101, 357-363. For the synthesis of 1c, $1 \mathbf{d}$ and $1 \mathbf{g}$ : R.-Q. Pana, X.-X. Liua, M.-Z. Deng, J. Fluorine Chem. 1999, 95, 167-170. For the synthesis of 1e and 1f: Y. Li, B. Zhao, K. Dai, D.-H. Tu, B. Wang, Y.-Y. Wang, Z.-T. Liu, Z.-W. Liu, J. Lu, Tetrahedron 2016, 72, 5684-5690. For the synthesis of 1h, 1i and 1l: O. Kobayashi, D. Uraguchi, T. Yamakawa, J. Fluorine Chem. 2009, 130, 591594. For the synthesis of $\mathbf{1 j}$ and $\mathbf{1 k}$ : T. A. Hamlin, C. B. Kelly, R. M. Cywar, N. E. Leadbeater, J. Org. Chem. 2014, 79, 1145-1155. For the synthesis of 1m: B. Jiang, Y. Xu, J. Org. Chem. 1991, 56, 7336-7340.

[2] V. Bizet, X. Pannecoucke, J.-L. Renaud, D. Cahard, J. Fluorine Chem. 2013, 152, 56-61.

[3] For the synthesis of 1u': M. Kimura, T. Yamazaki, T. Kitazume, T. Kubota, Org. Lett. 2004, 6, 4651-4654. For the synthesis of 1y: Y. Chen, L. Huang, X. P. Zhang, J. Org. Chem. 2003, 68, 5925-5929. For the synthesis of 1z: R.-Q. Pan, P.-A. Wang, M.-Z. Deng, Heteroat. Chem. 2002, 13, 287-290. For the synthesis of 1aa, 1ac and 1ad: T. Konno, T. Takehana, M. Mishima, T. Ishihara, J. Org. Chem. 2006, 71, 3545-3550. For the synthesis of 1ab: Y. Chen, L. Huang, X. P. Zhang, J. Org. Chem. 2003, 68, 5925-5929. For the synthesis of 1ae, 1af, 1ag and 1ai: P. Poutrel, M. V. Ivanova, X. Pannecoucke, P. Jubault, T. Poisson, Chem. Eur. J. 2019, 25, 15262-15266.

[4] S. Kerdphon, S. Ponra, J. Yang, H. Wu, L. Eriksson, P. G. Andersson, ACS Catal. 2019, 9, 61696176. 


\section{NMR spectra}

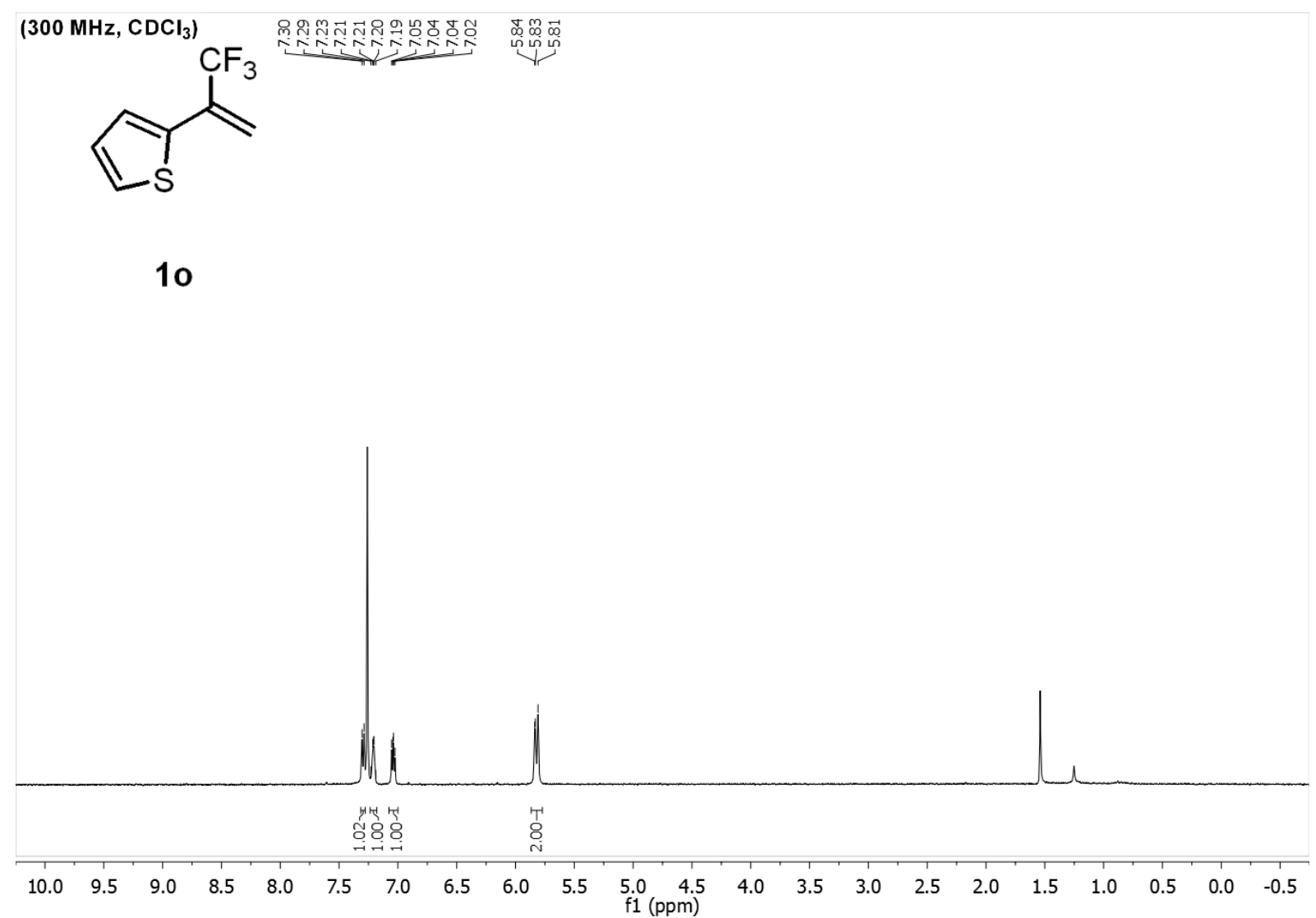

$\left(75 \mathrm{MHz}, \mathrm{CDCl}_{3}\right)$

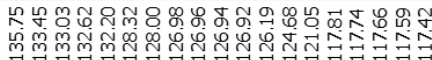

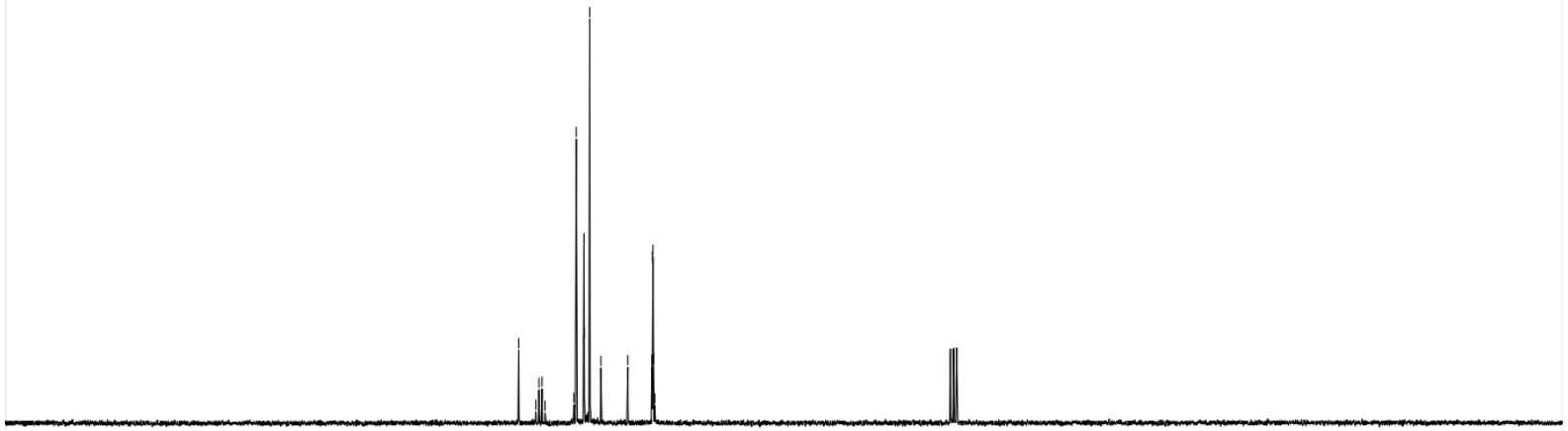

$\begin{array}{lllllllllllllllllllll}200 & 190 & 180 & 170 & 160 & 150 & 140 & 130 & 120 & 110 & 100 & 90 & 80 & 70 & 60 & 50 & 40 & 30 & 20 & 10 & 0\end{array}$ 
(282 MHz, $\mathrm{CDCl}_{3}$ )

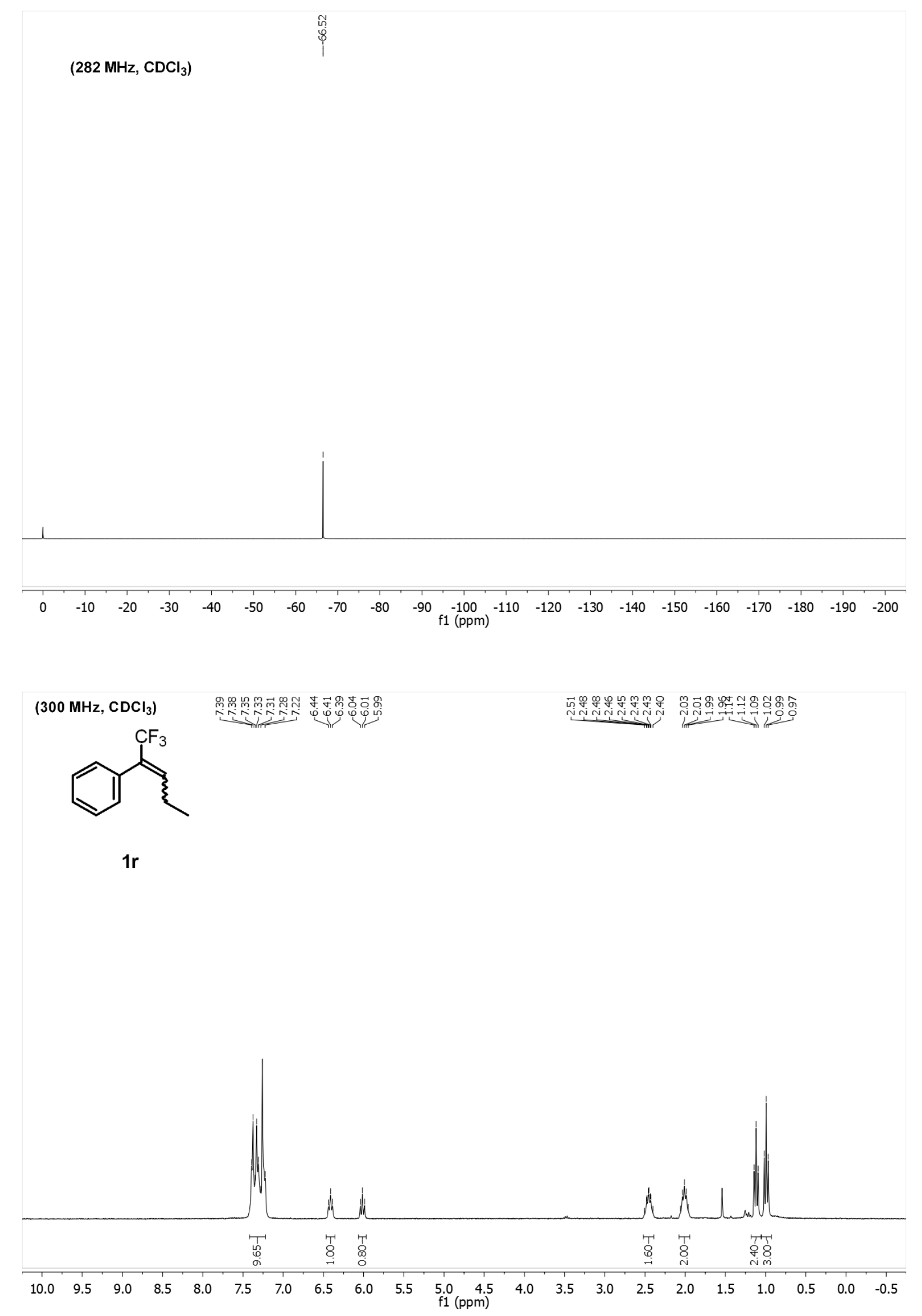




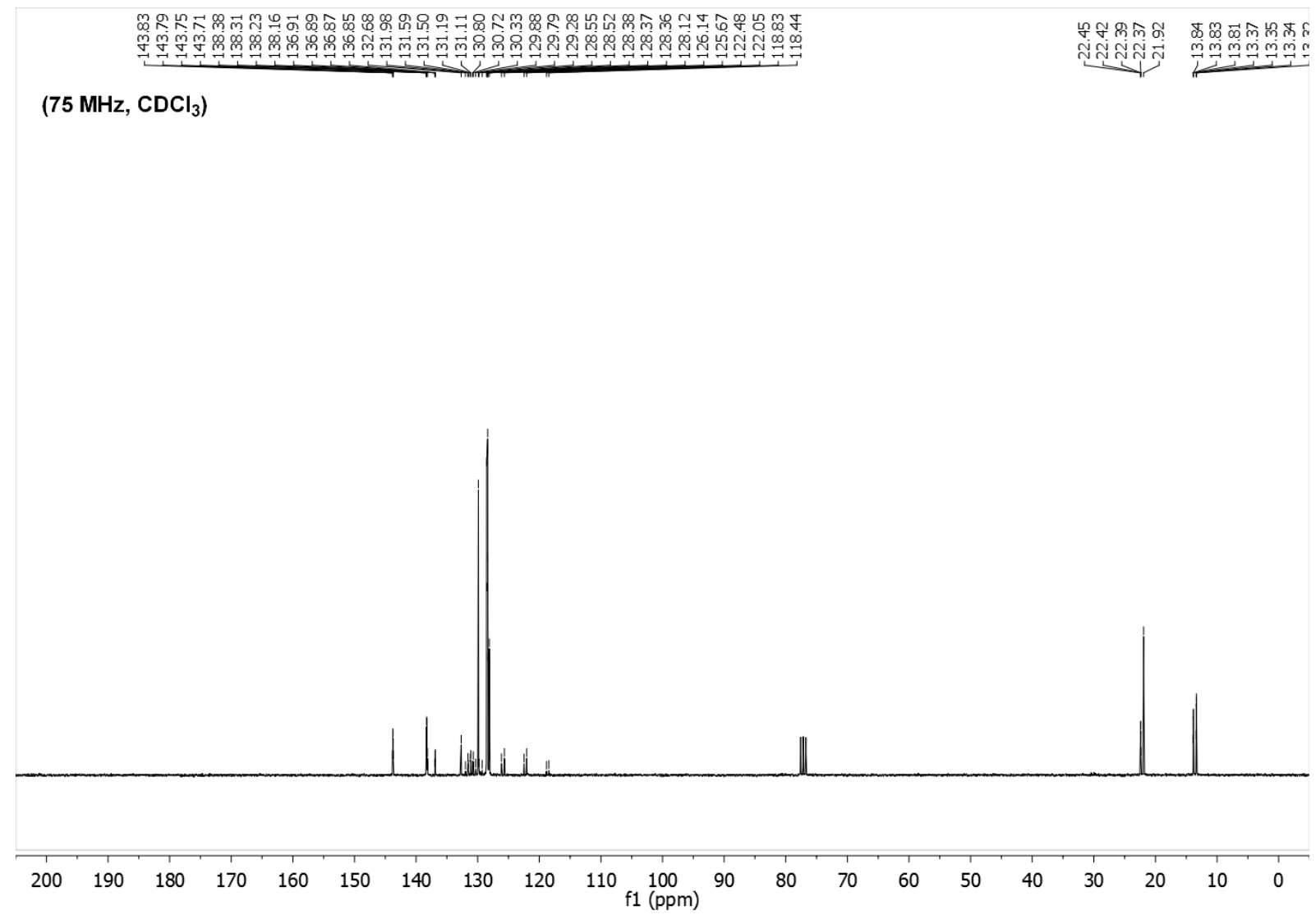

(282 MHz, $\mathrm{CDCl}_{3}$ )
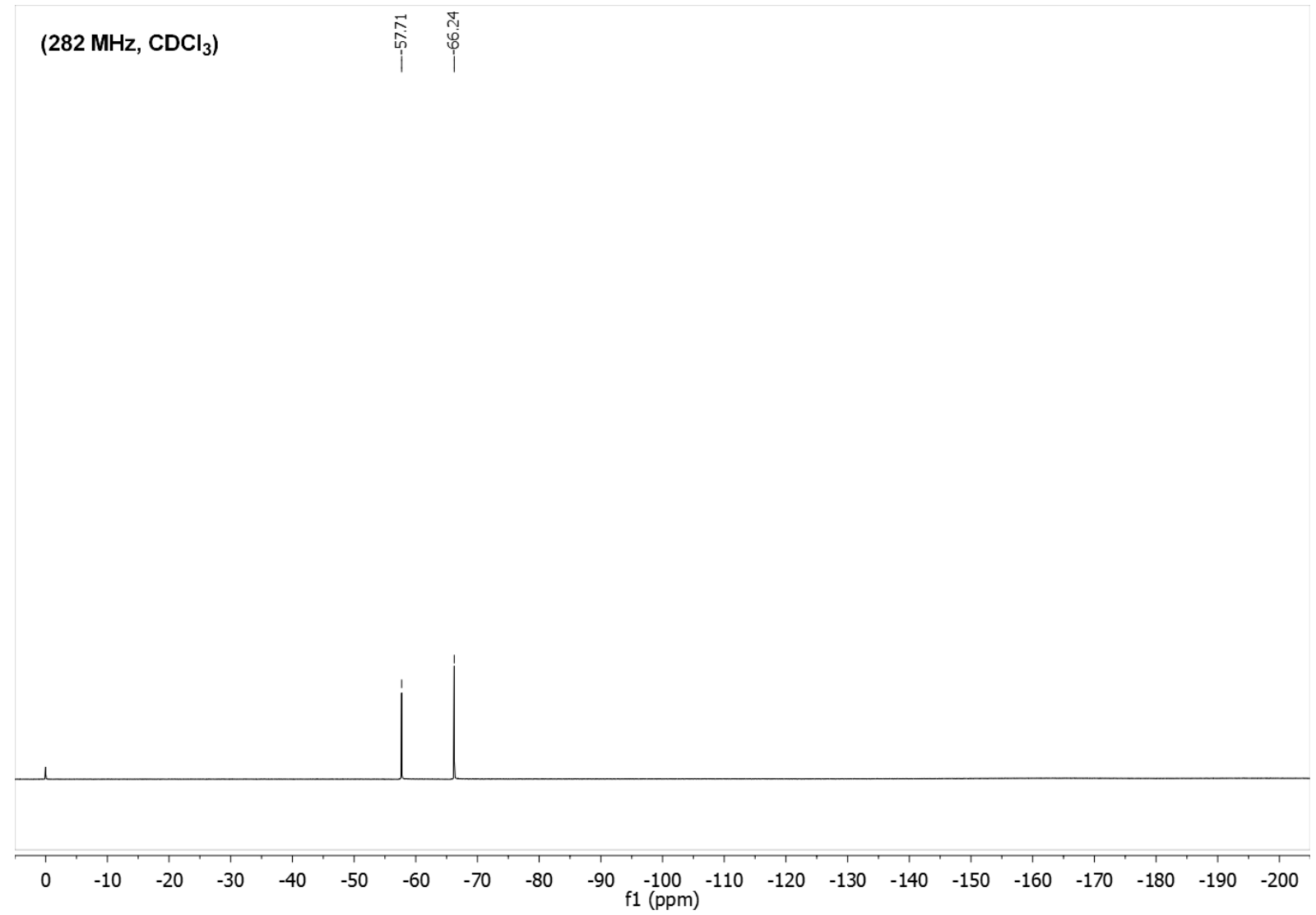

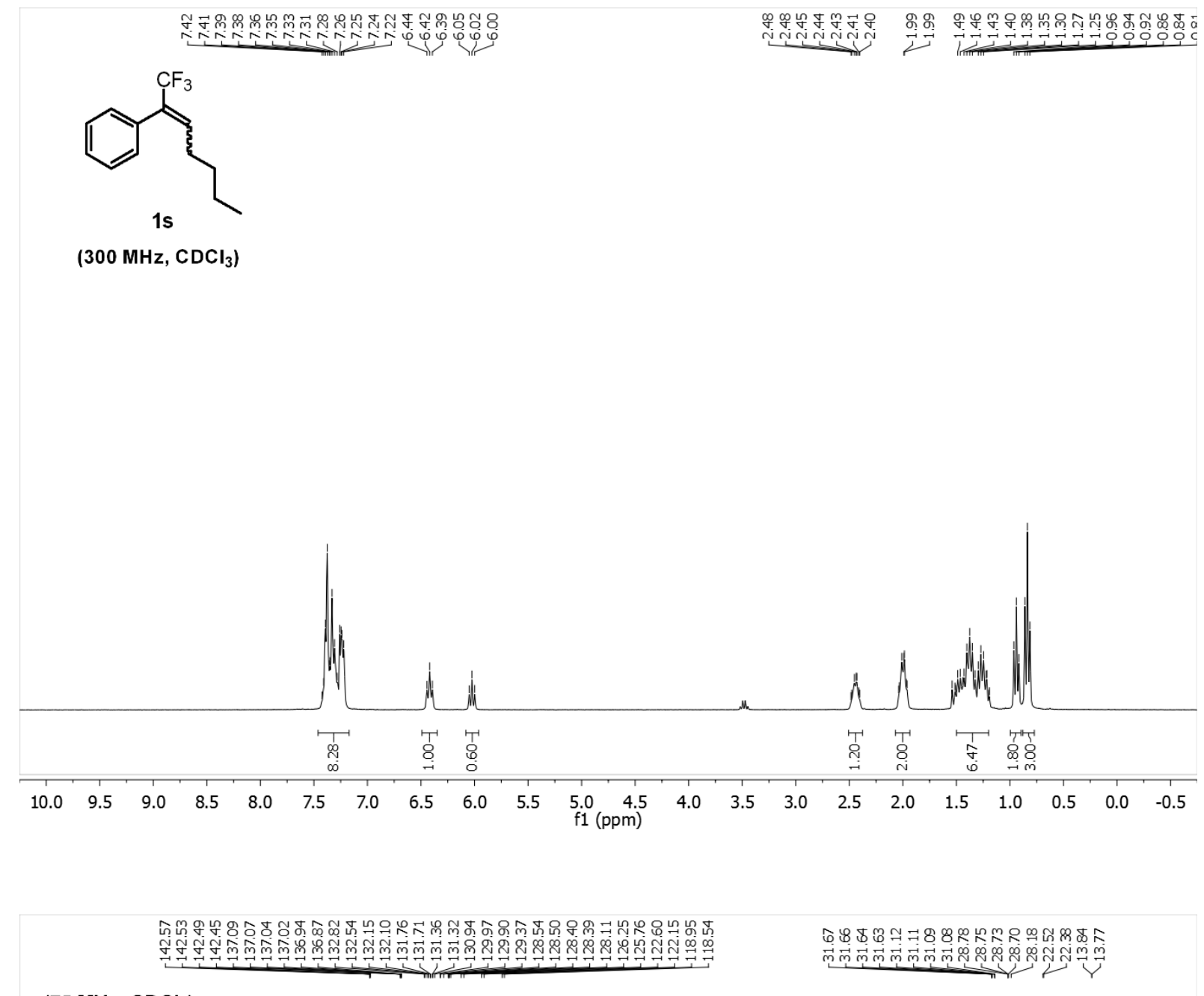

(75 MHz, $\mathrm{CDCl}_{3}$ )

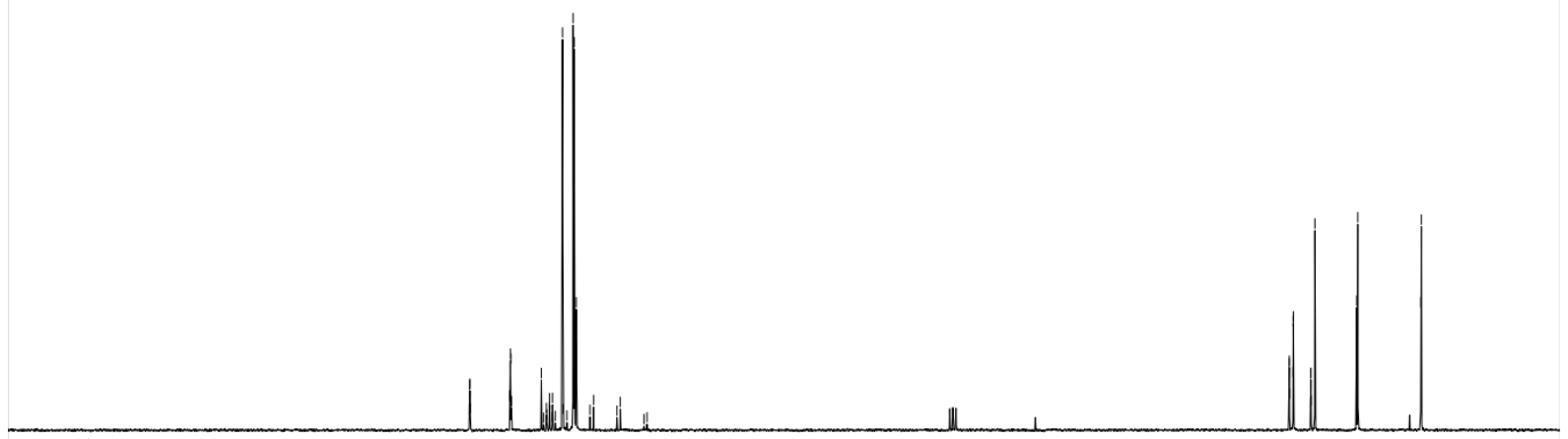


(282 MHz, $\mathrm{CDCl}_{3}$ )
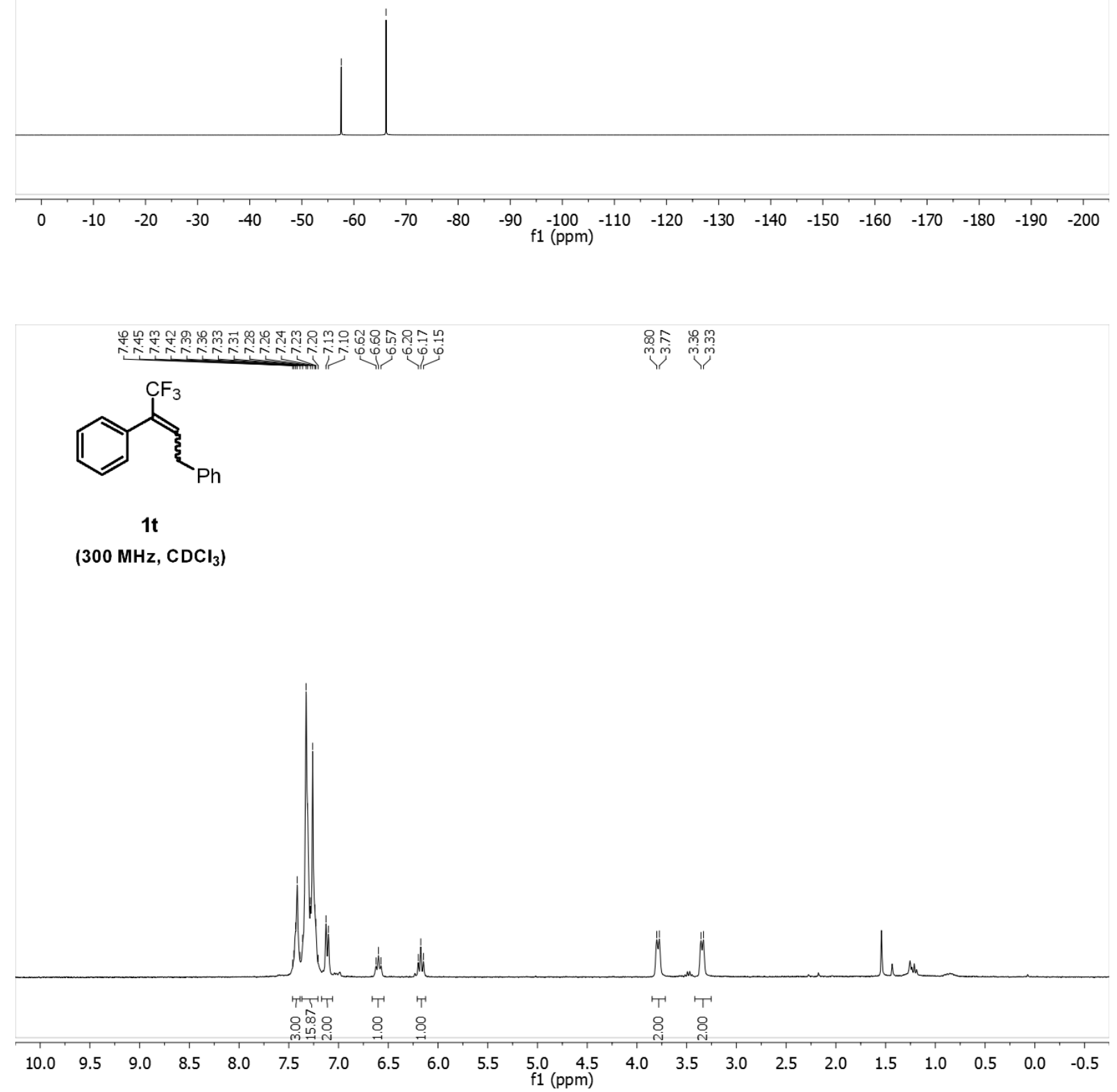
$\left(75 \mathrm{MHz}, \mathrm{CDCl}_{3}\right)$

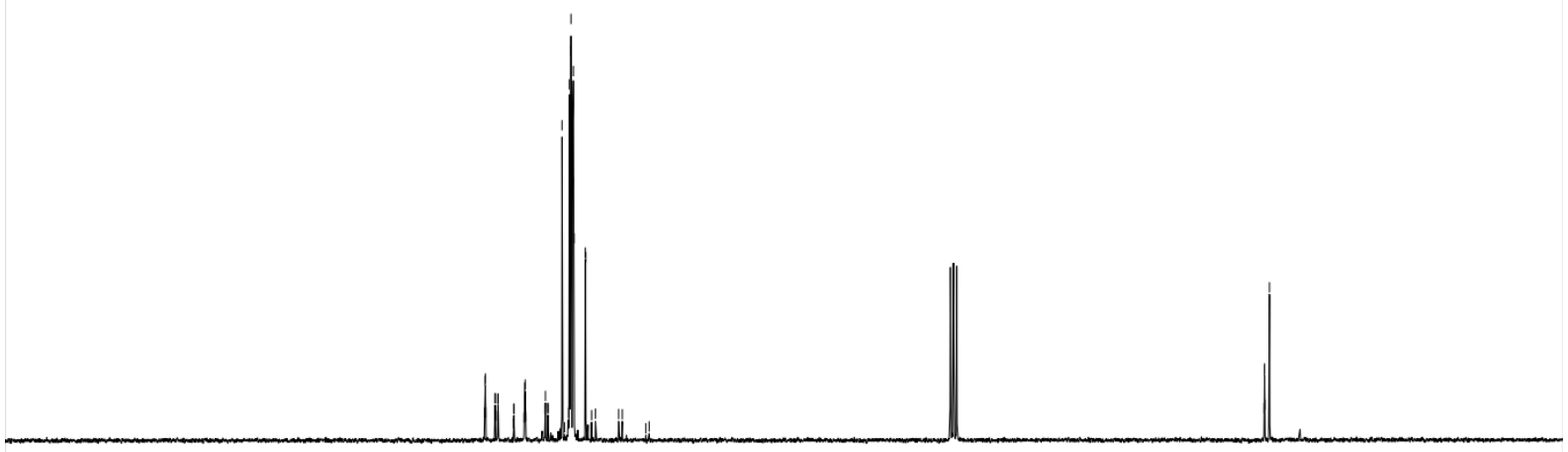

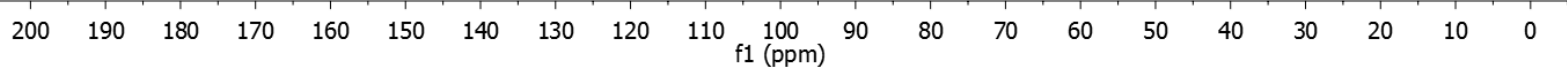

$\left(282 \mathrm{MHz}, \mathrm{CDCl}_{3}\right)$

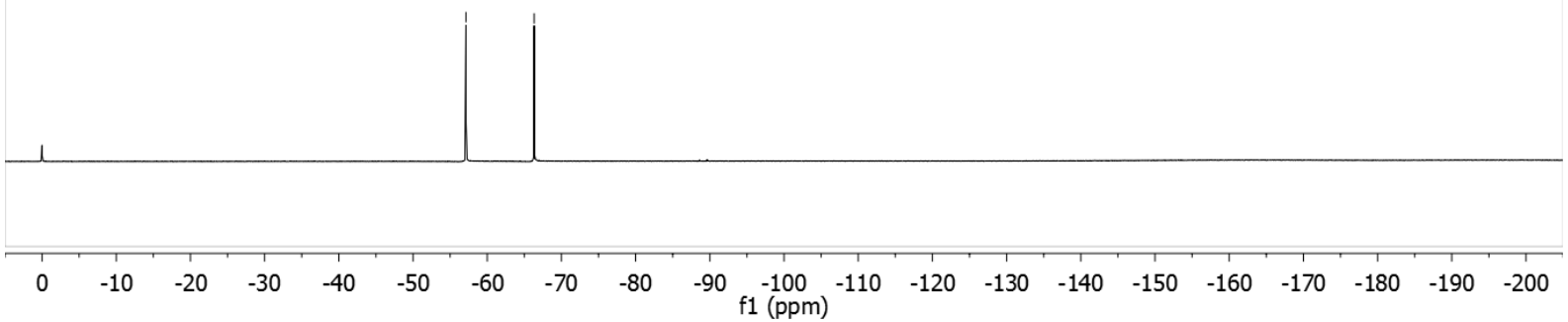




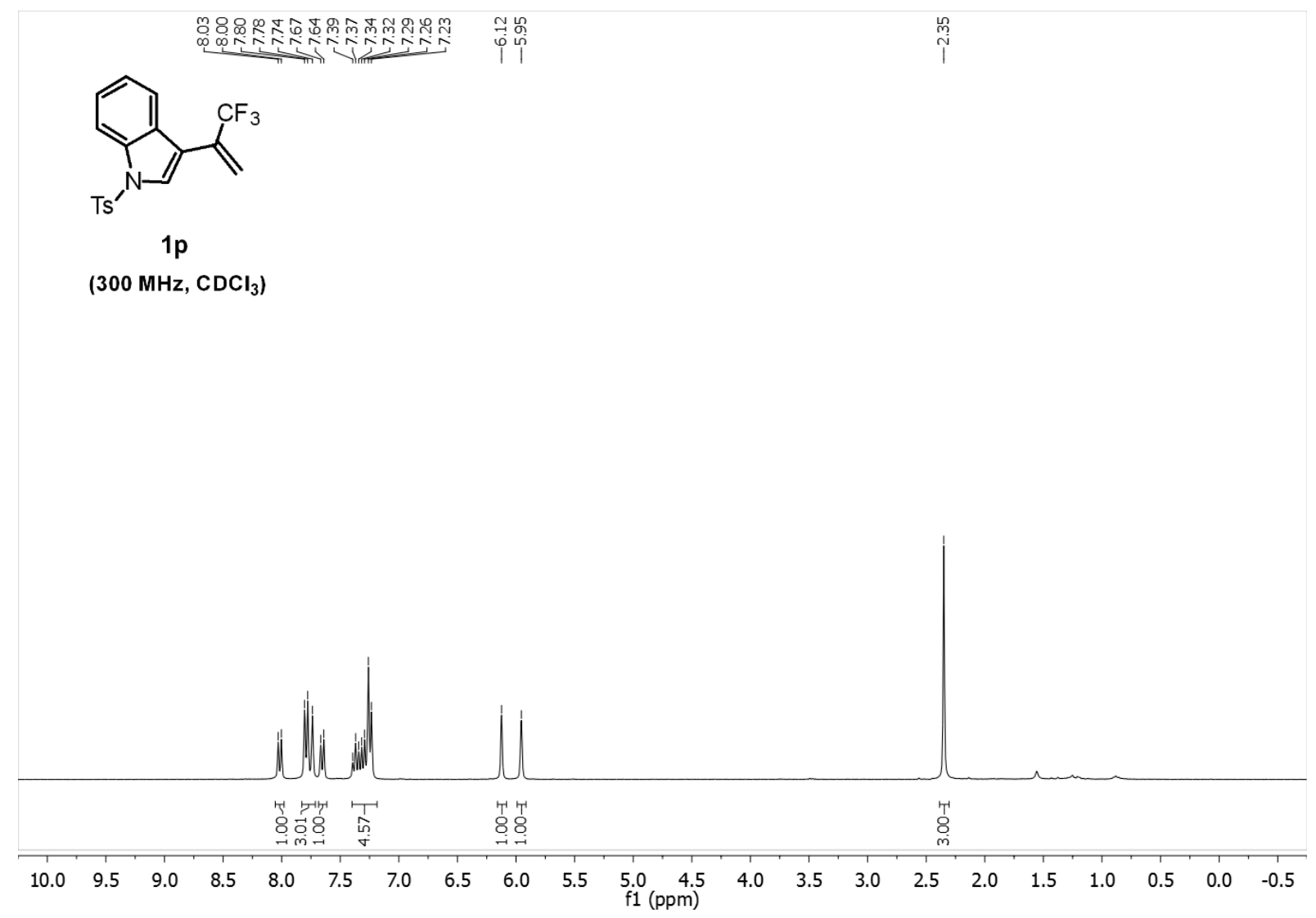

(75 $\mathrm{MHz}, \mathrm{CDCl}_{3}$ )

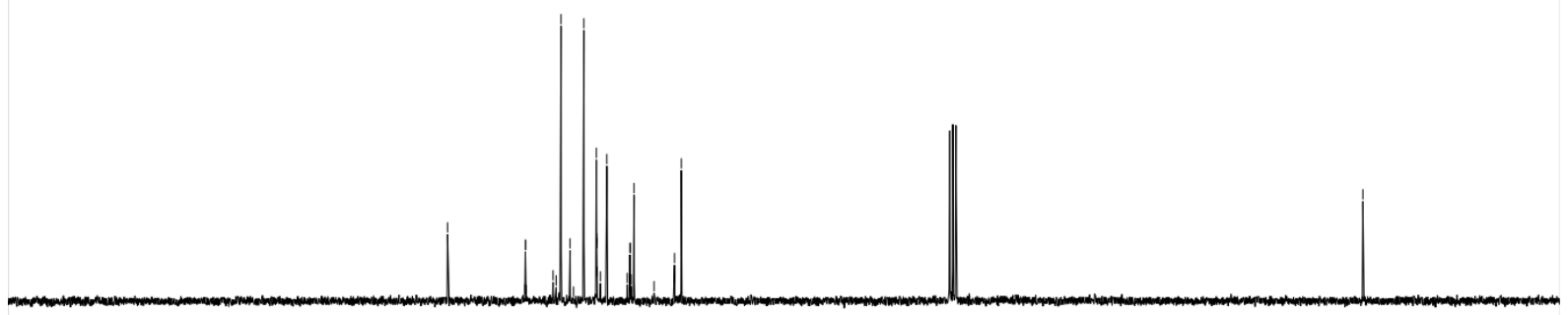


(282 MHz, $\mathrm{CDCl}_{3}$ )

$$
\stackrel{ }{\stackrel{9}{\varphi}}
$$

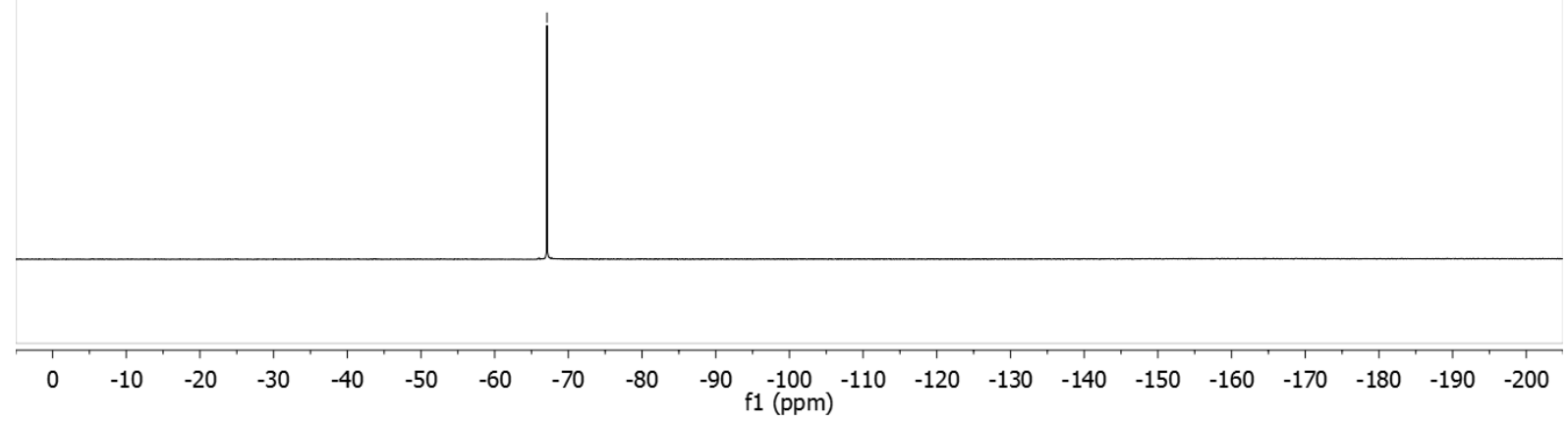<smiles></smiles>

$1 q$

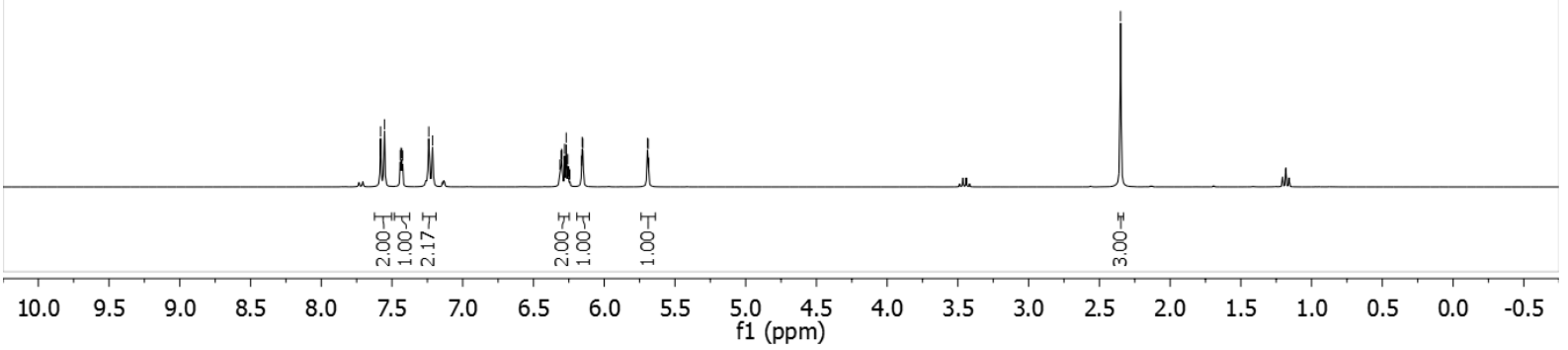




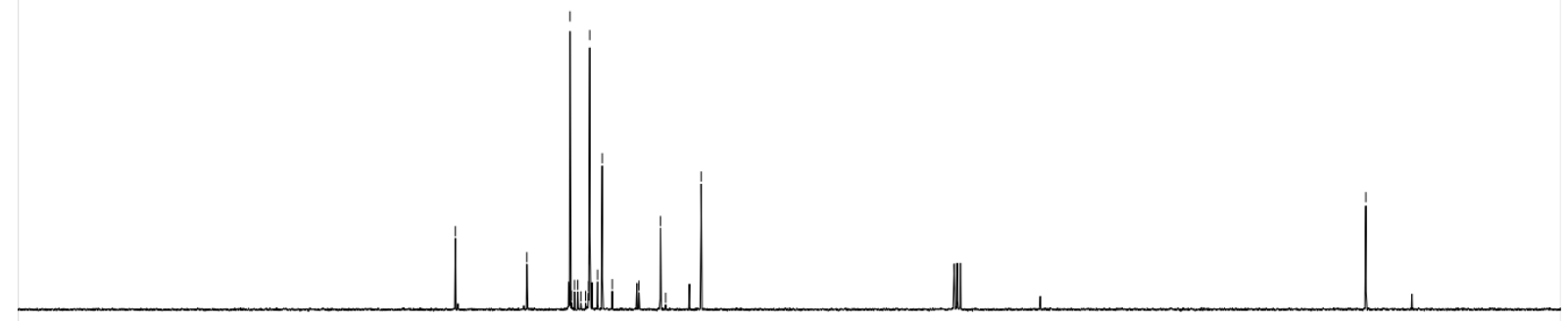

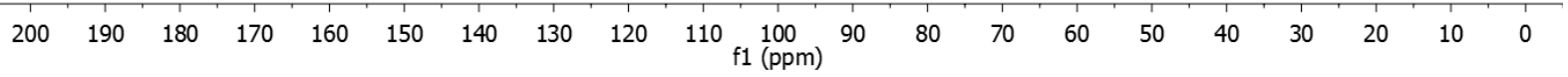

(282 MHz, $\mathrm{CDCl}_{3}$ )

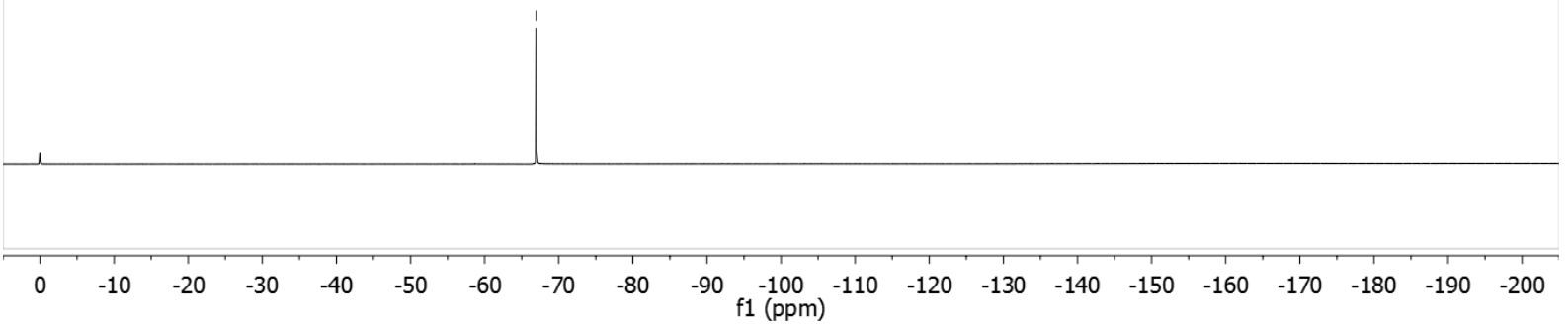



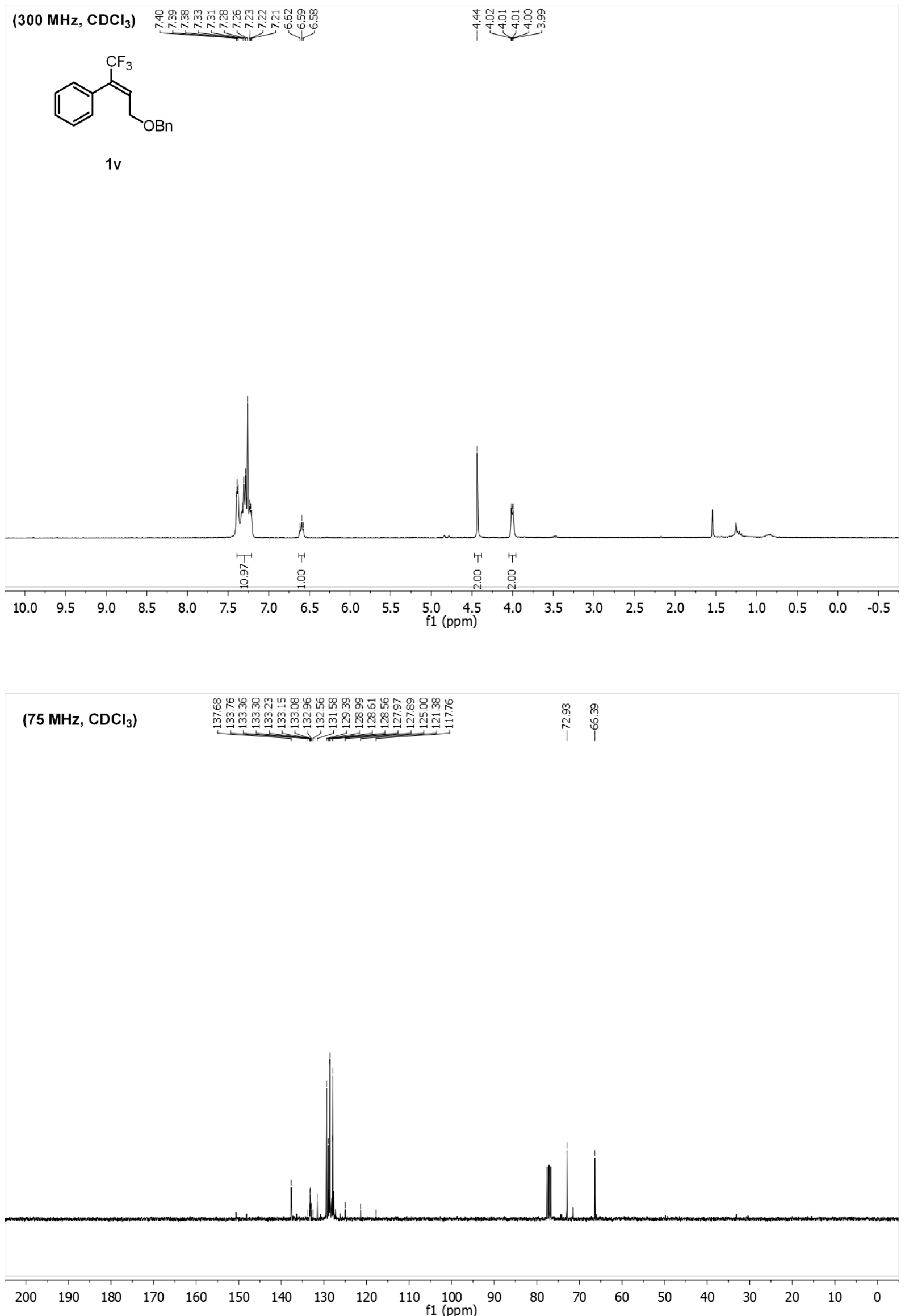
(282 MHz, $\mathrm{CDCl}_{3}$ )

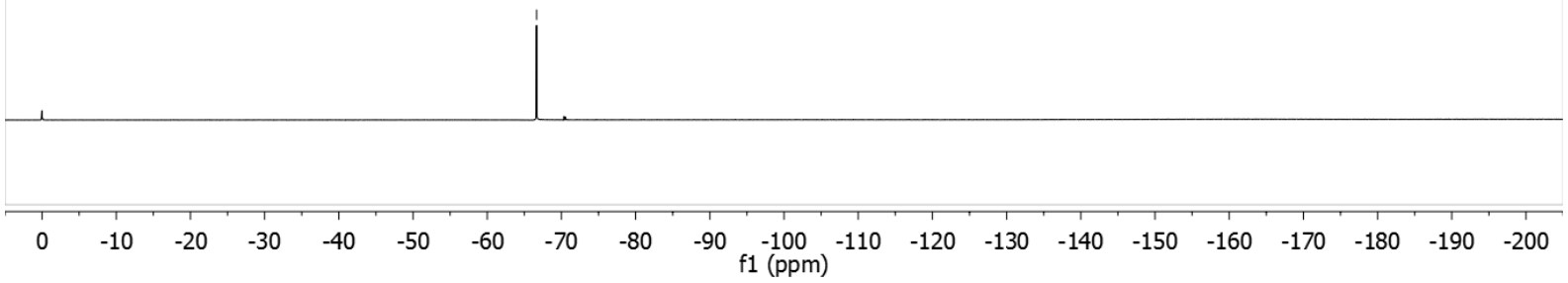

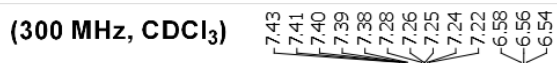

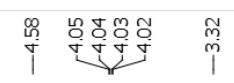

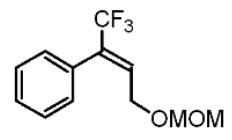

$1 w$

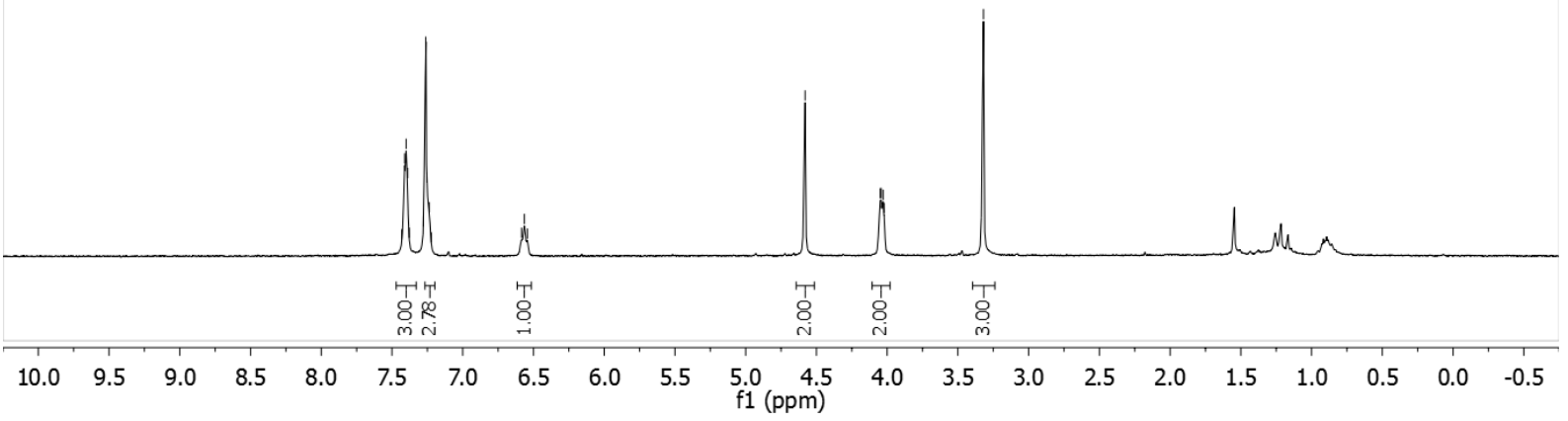




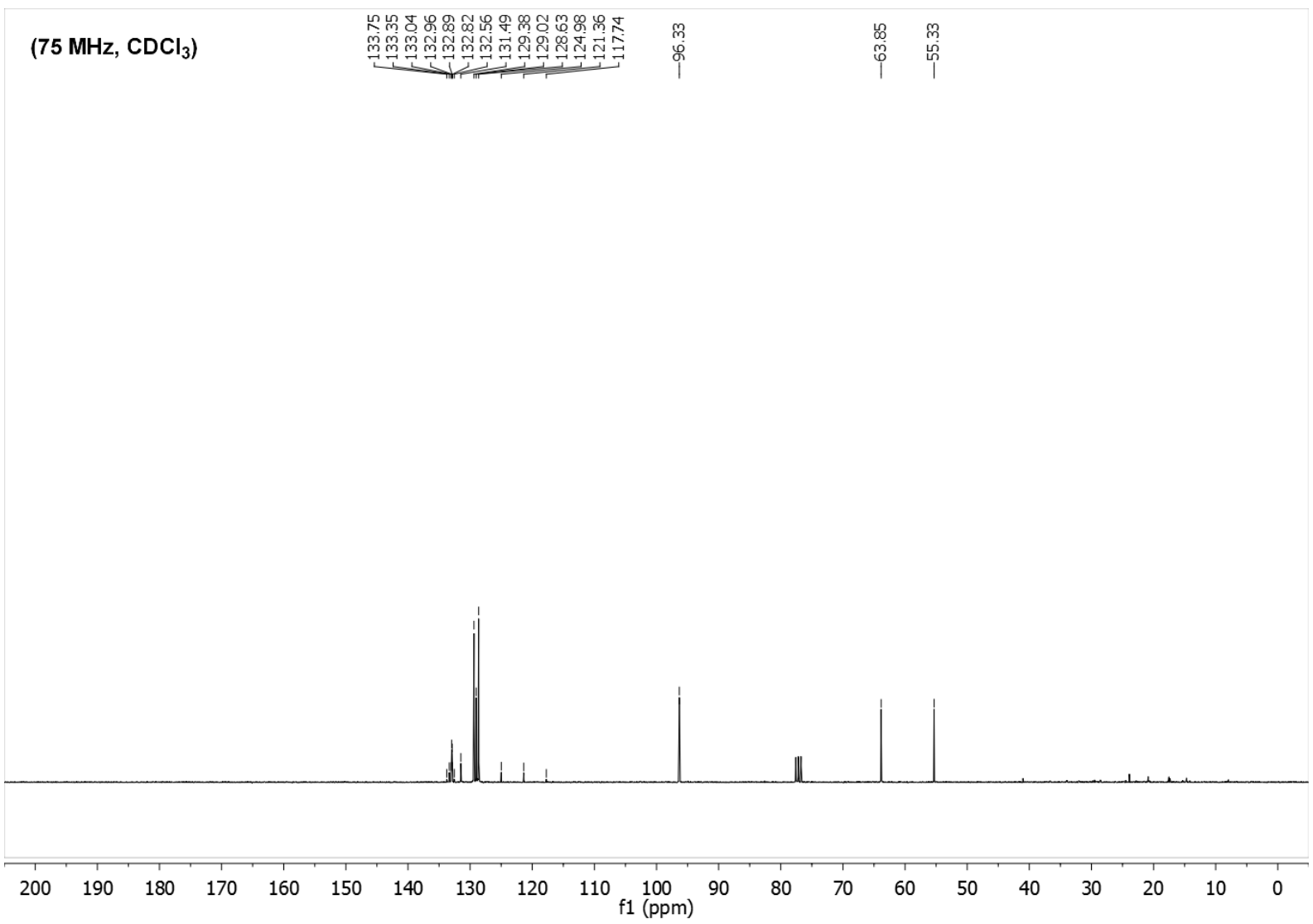

(282 MHz, $\mathrm{CDCl}_{3}$ )

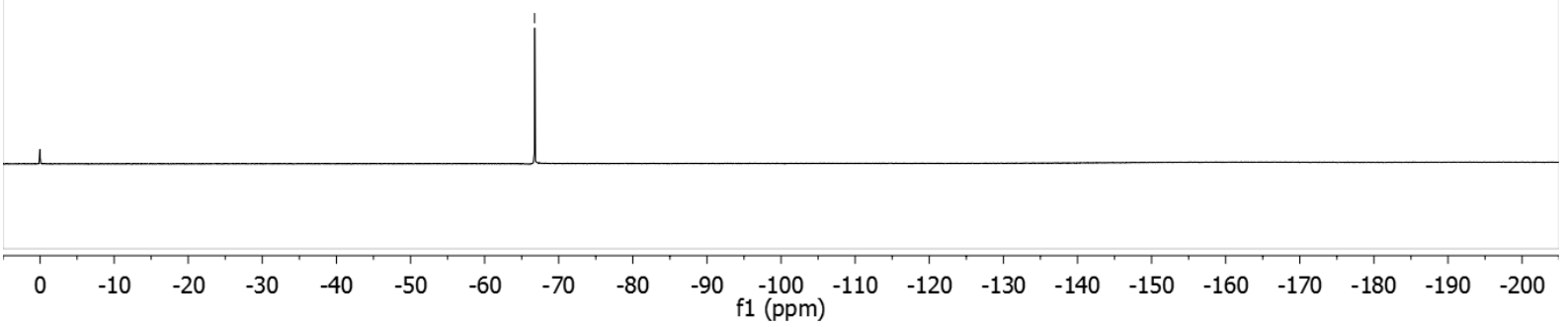




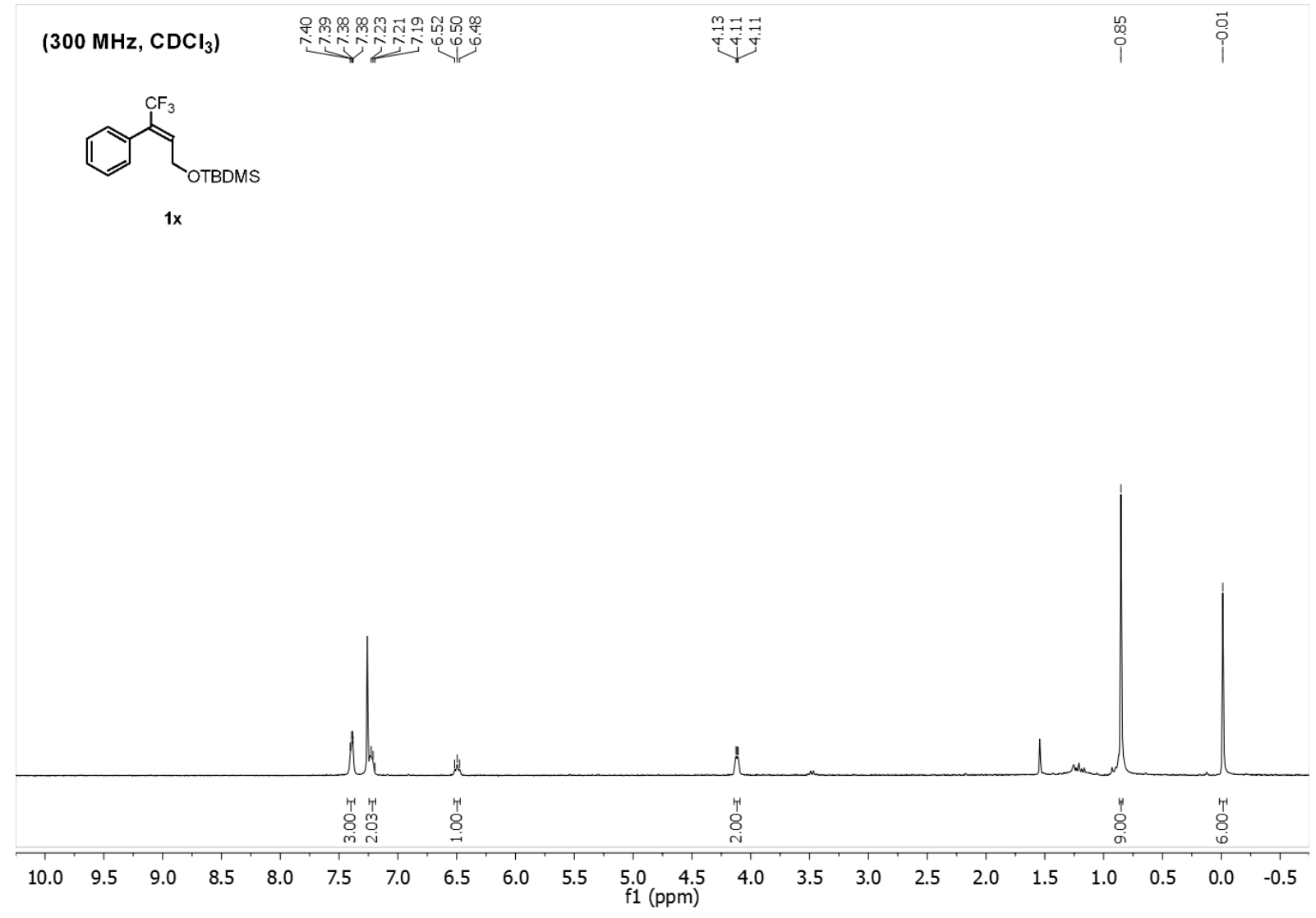

$\left(75 \mathrm{MHz}, \mathrm{CDCl}_{3}\right)$

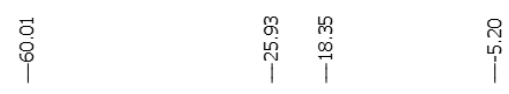

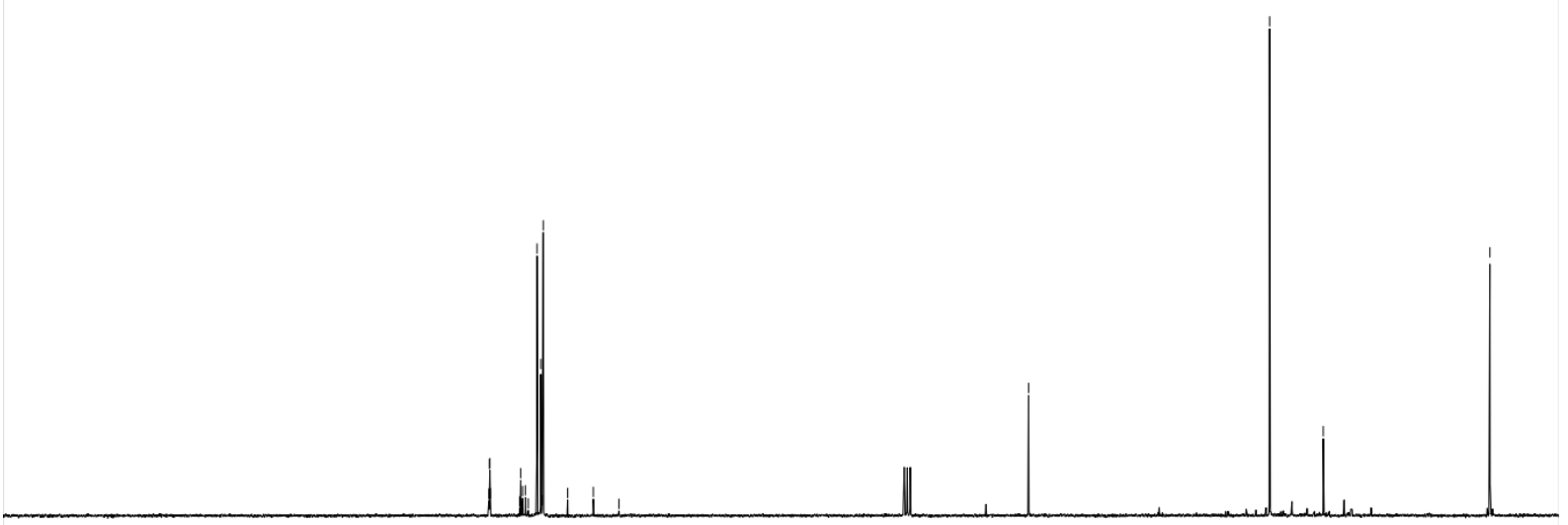

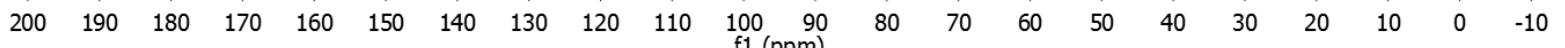


(282 $\mathrm{MHz}, \mathrm{CDCl}_{3}$ )

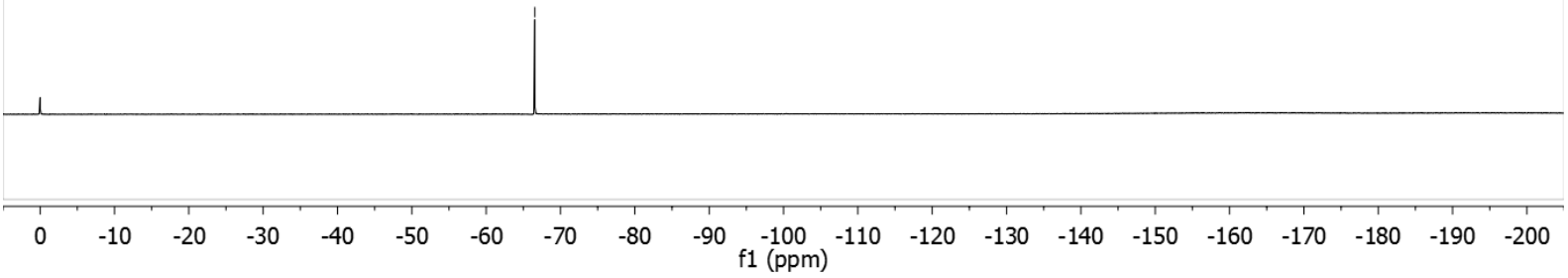

$\left(300 \mathrm{MHz}, \mathrm{CDCl}_{3}\right) \underbrace{m}$<smiles>C=C(C=C(OCC)c1cccc(Cl)c1)C(F)(F)F</smiles>

1ah

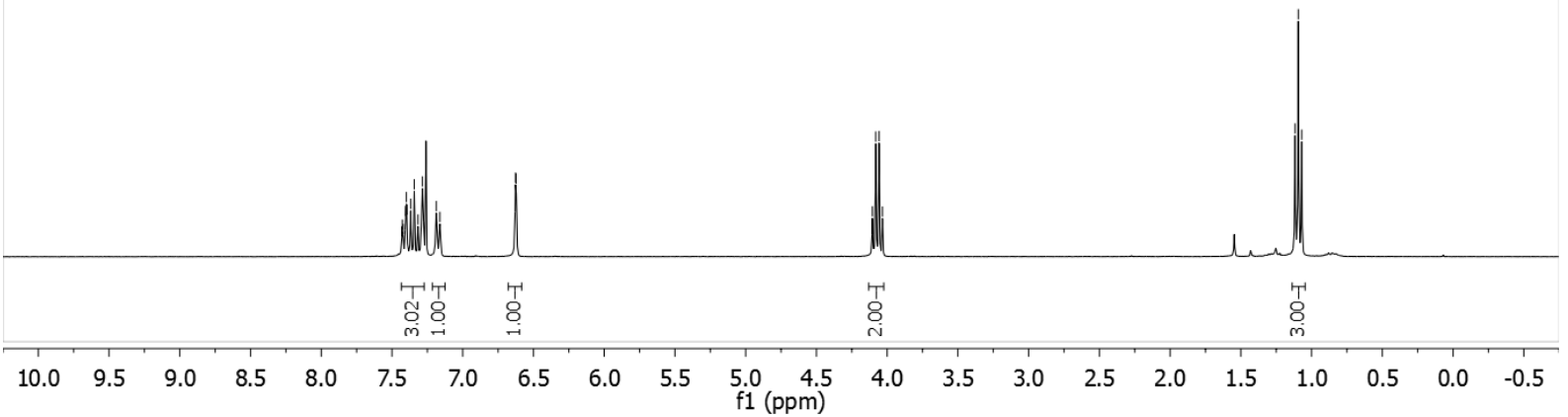




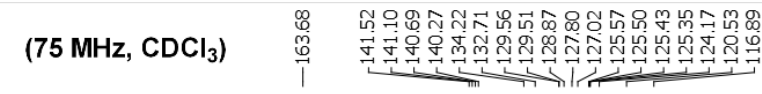

$\stackrel{\substack{i \\ i}}{\stackrel{0}{i}}$

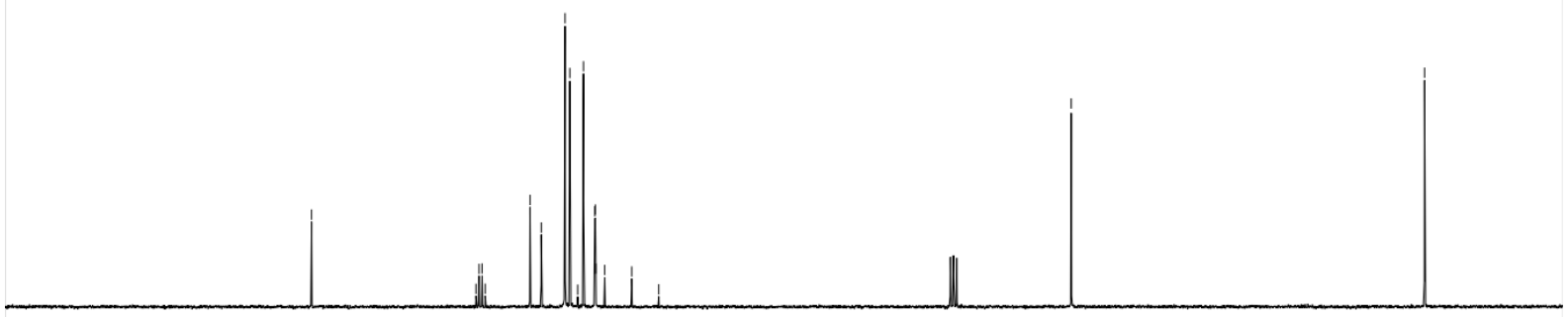

$\begin{array}{lllllllllllllllllllll}200 & 190 & 180 & 170 & 160 & 150 & 140 & 130 & 120 & 110 & 100 & 90 & 80 & 70 & 60 & 50 & 40 & 30 & 20 & 10 & 0\end{array}$

$\left(282 \mathrm{MHz}, \mathrm{CDCl}_{3}\right)$

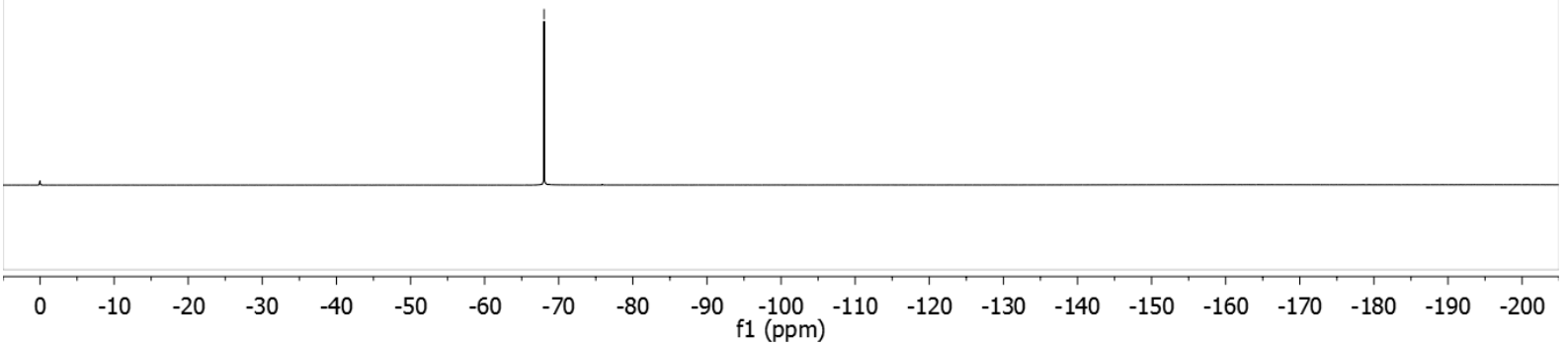




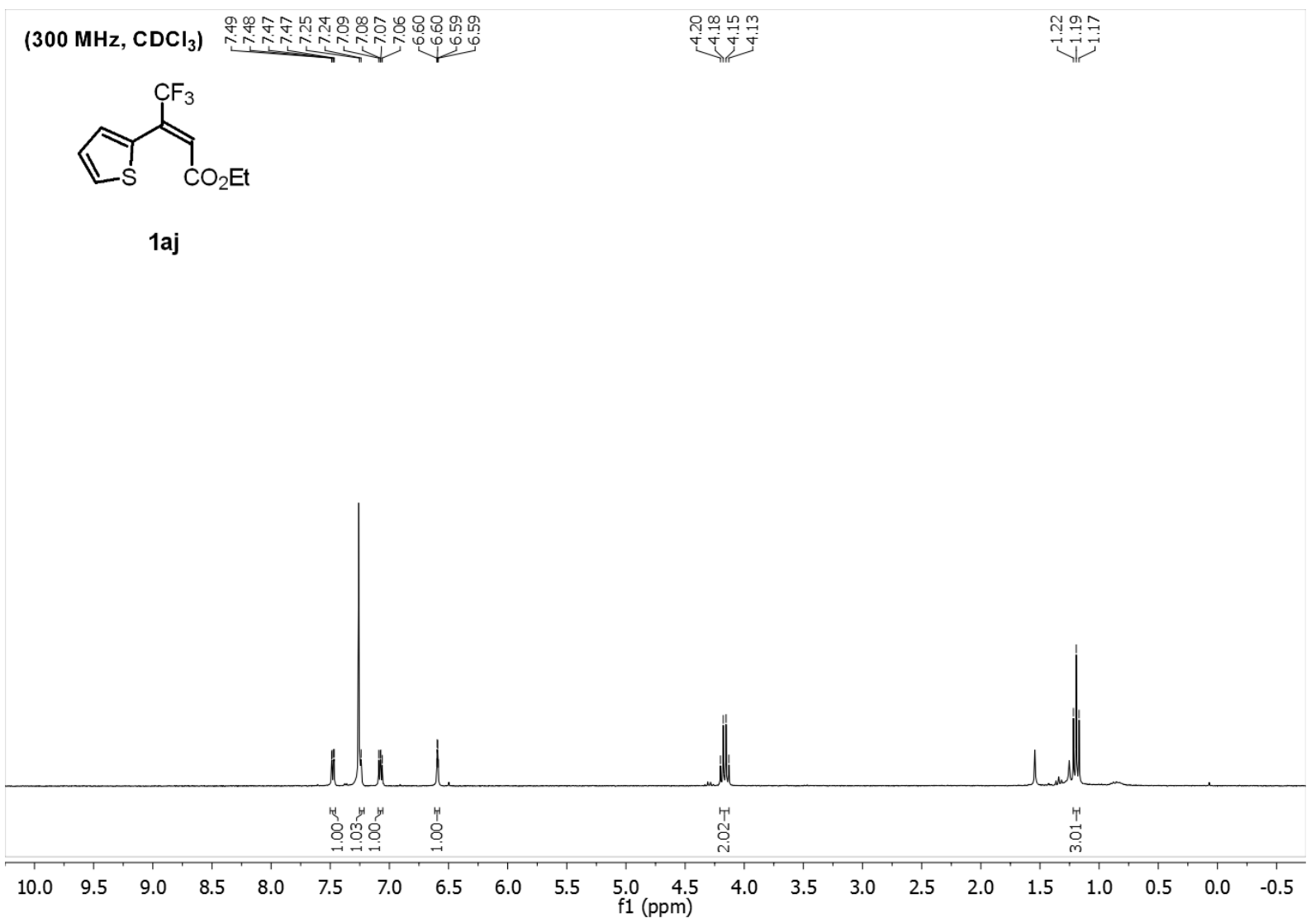

$\left(75 \mathrm{MHz}, \mathrm{CDCl}_{3}\right)$ 誉
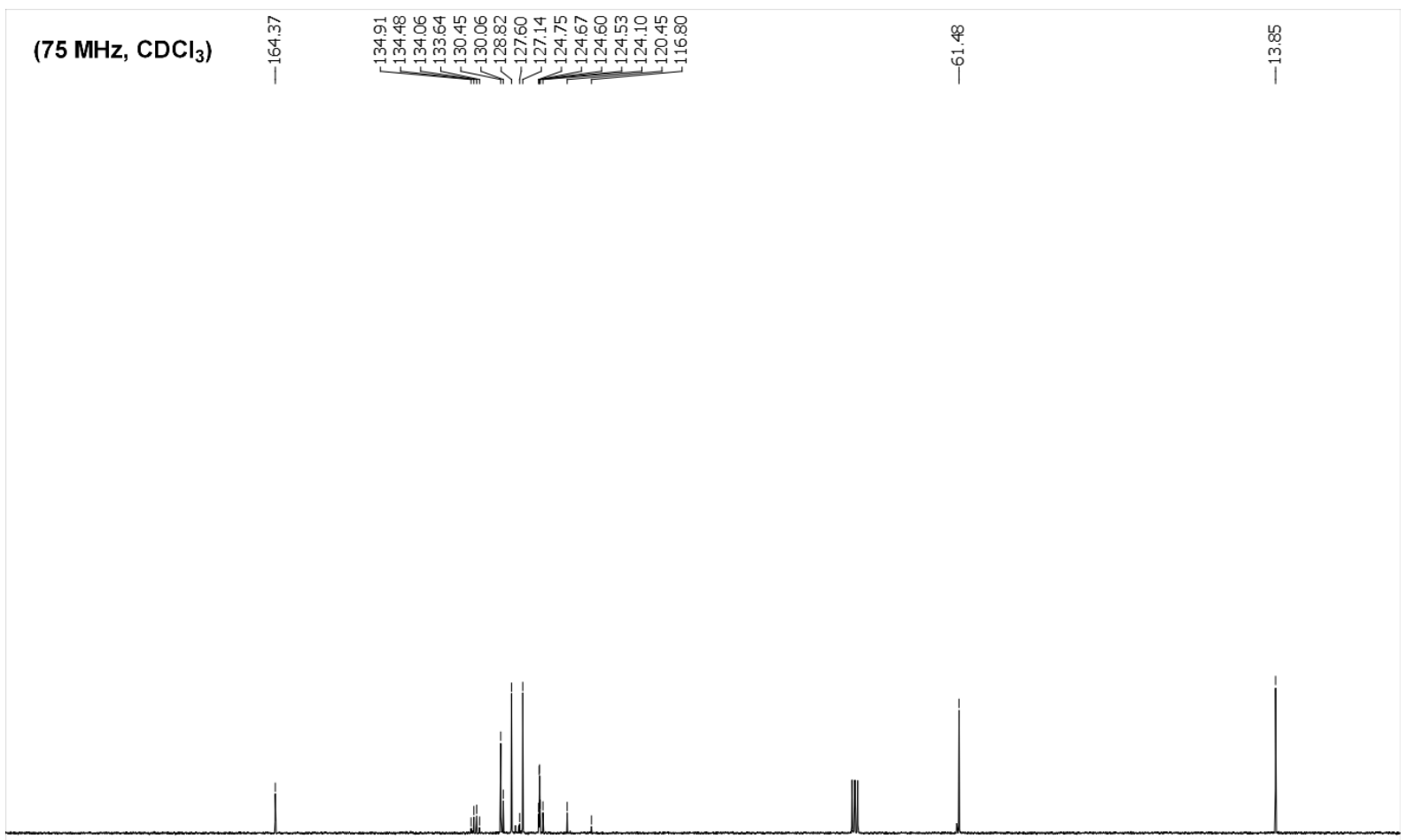

$\begin{array}{llllllllllllllllllllllll}200 & 190 & 180 & 170 & 160 & 150 & 140 & 130 & 120 & 110 & 100 & 90 & 80 & 70 & 60 & 50 & 40 & 30 & 20 & 10 & 0\end{array}$ 
(282 MHz, $\mathrm{CDCl}_{3}$ )
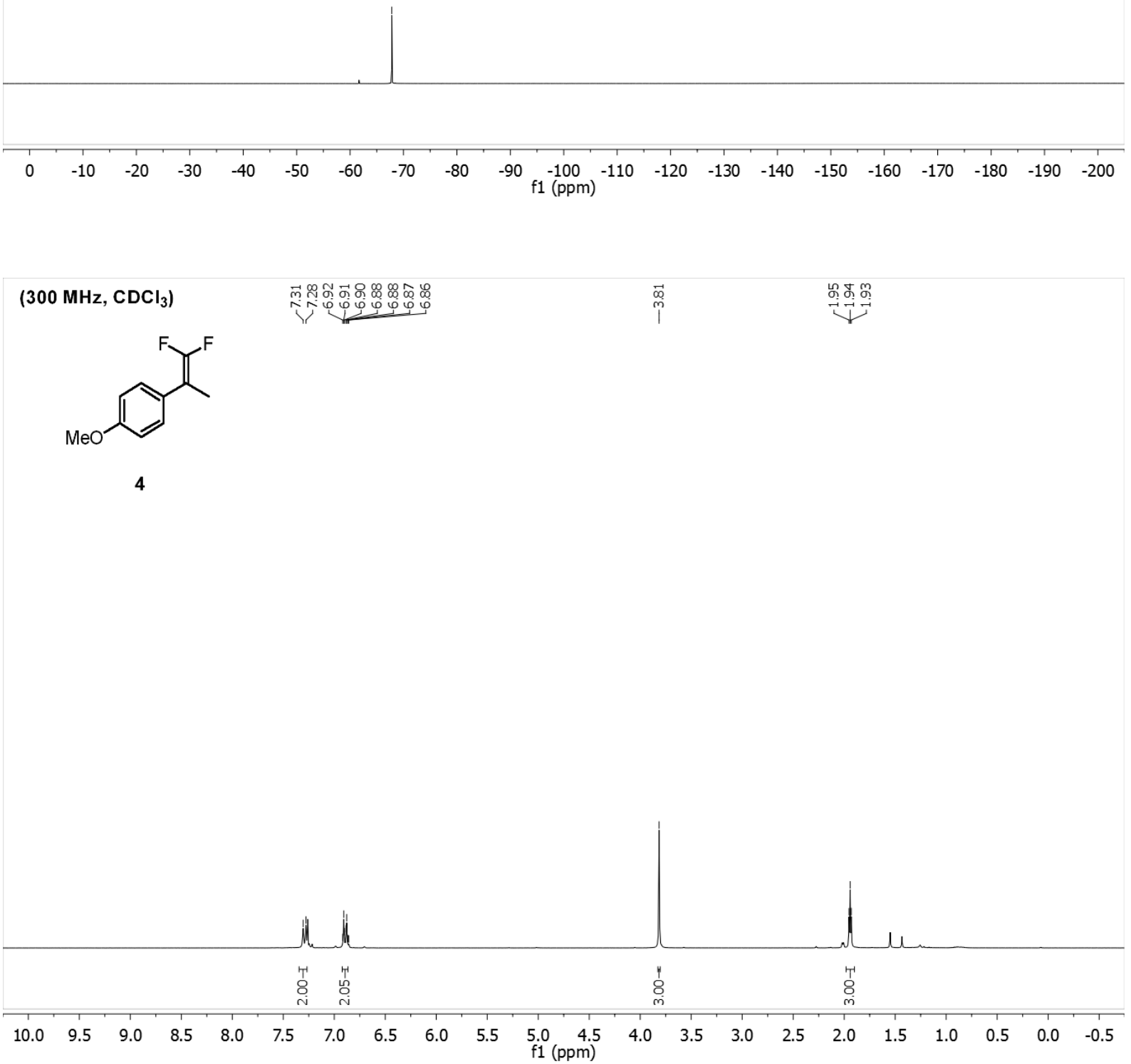


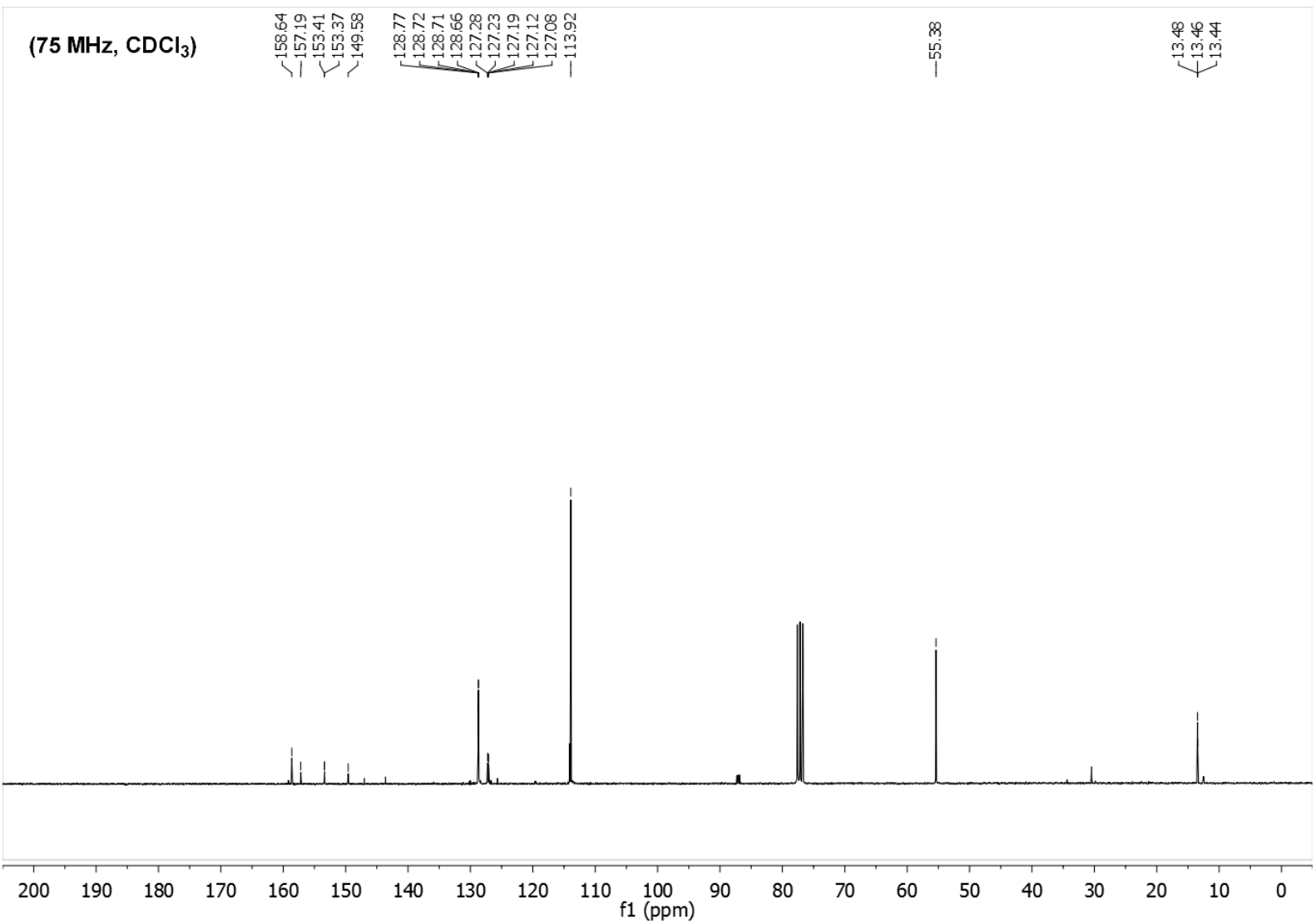

(282 $\mathrm{MHz}, \mathrm{CDCl}_{3}$ )
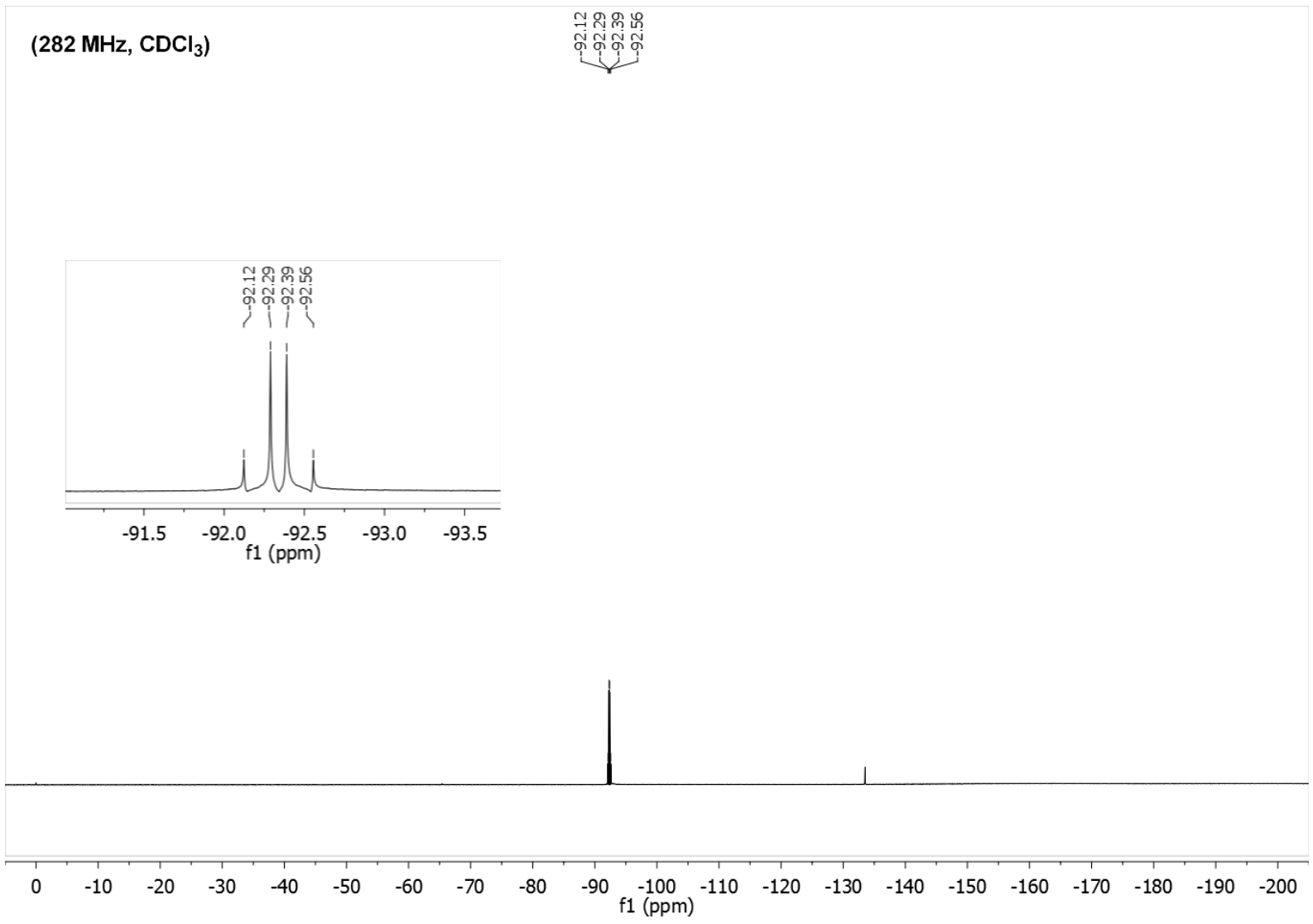


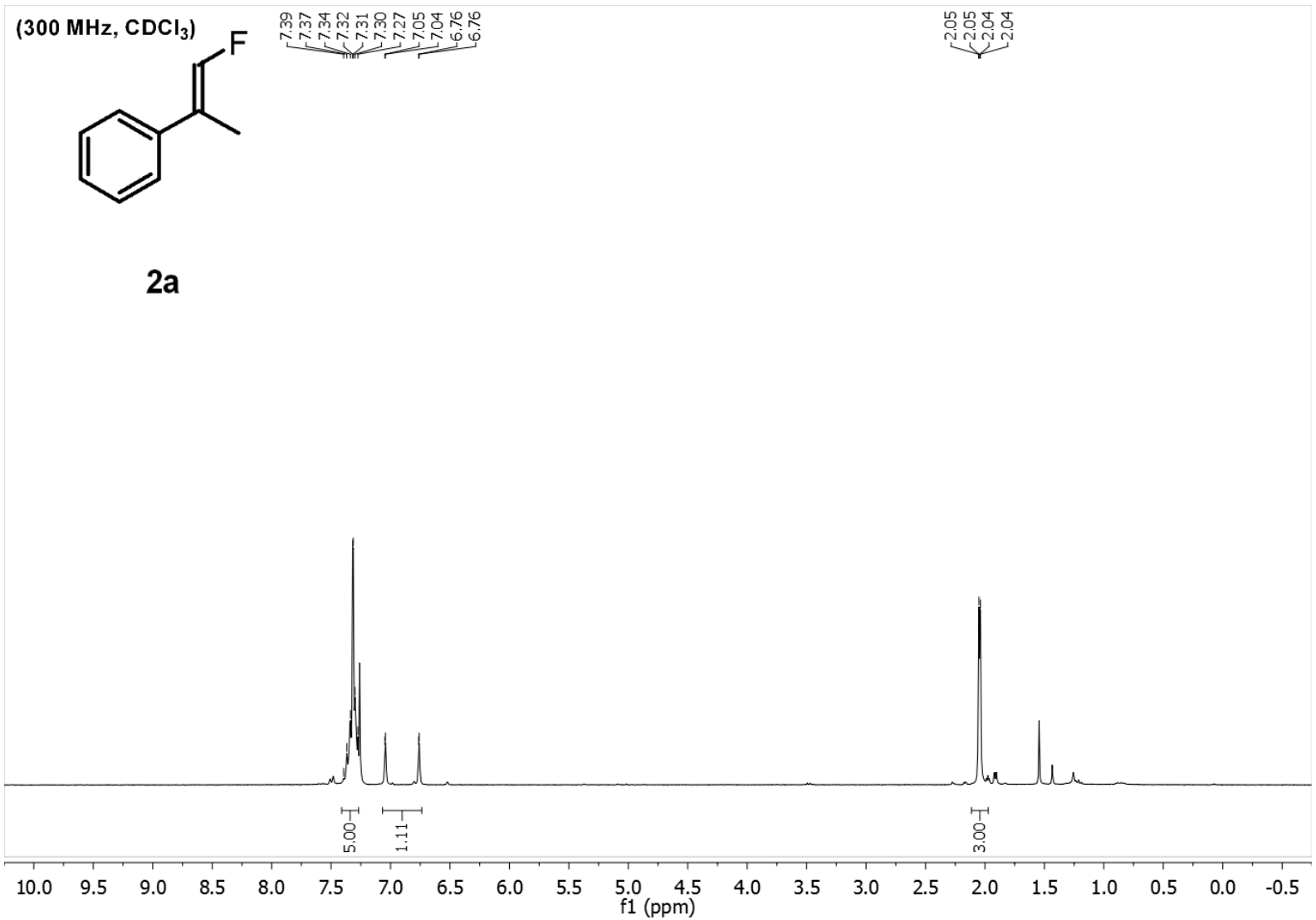

(75 MHz, $\mathrm{CDCl}_{3}$ )
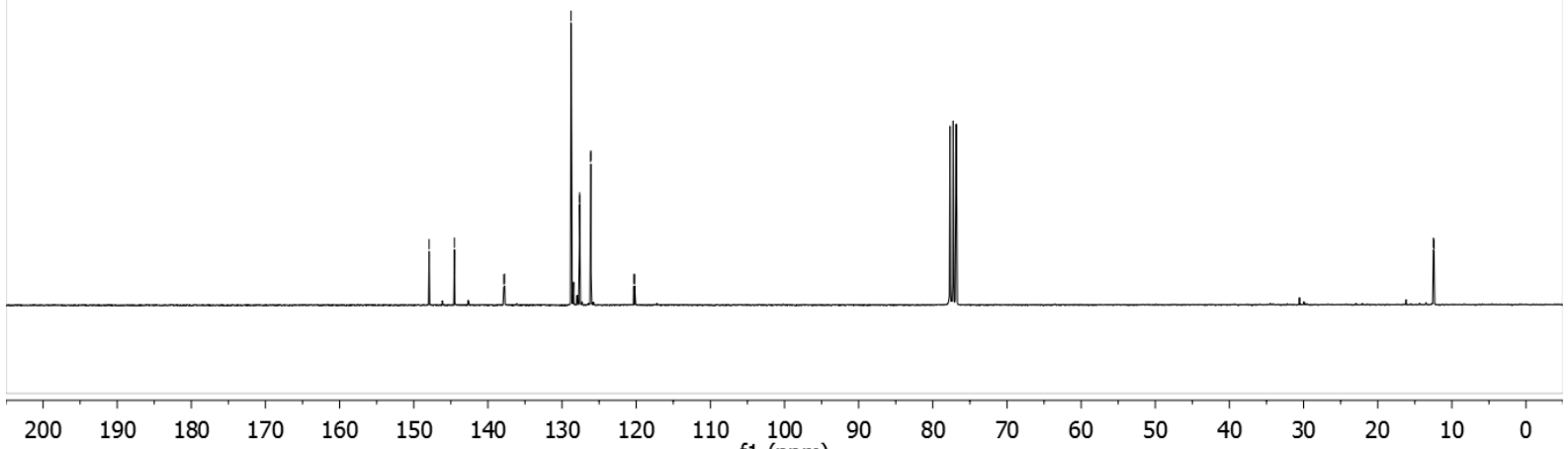
(282 MHz, $\mathrm{CDCl}_{3}$ )

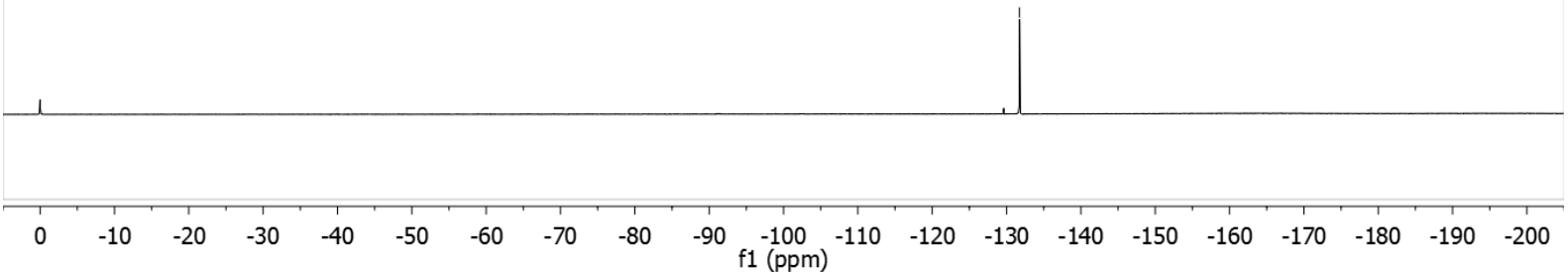<smiles>CC(=O)OCC(C)(C)C(=O)O</smiles>

2b

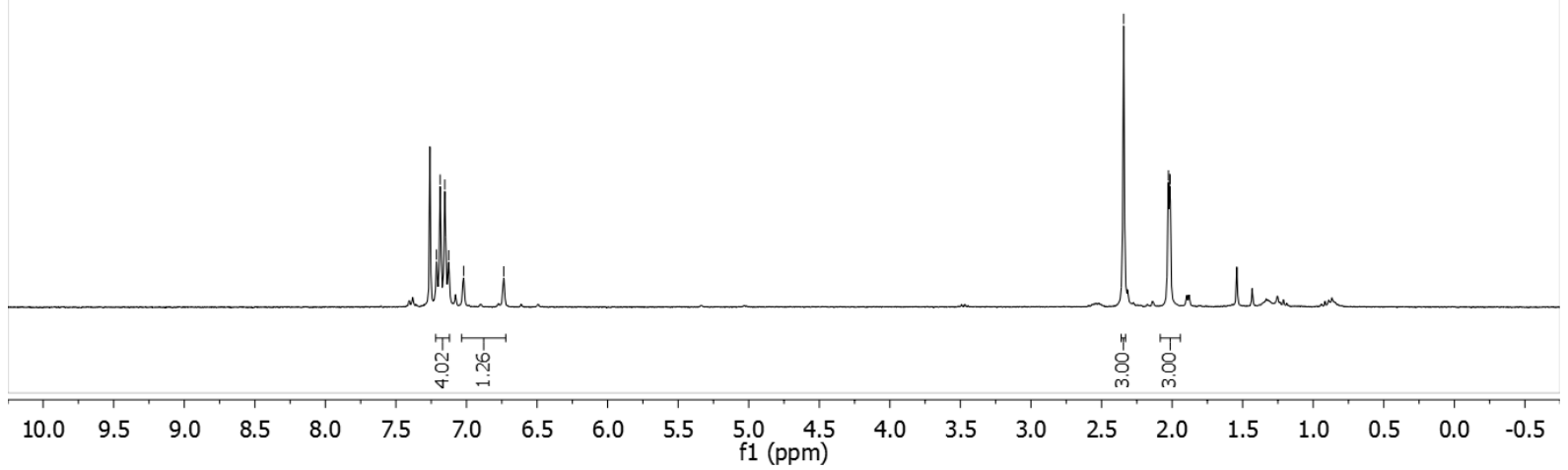




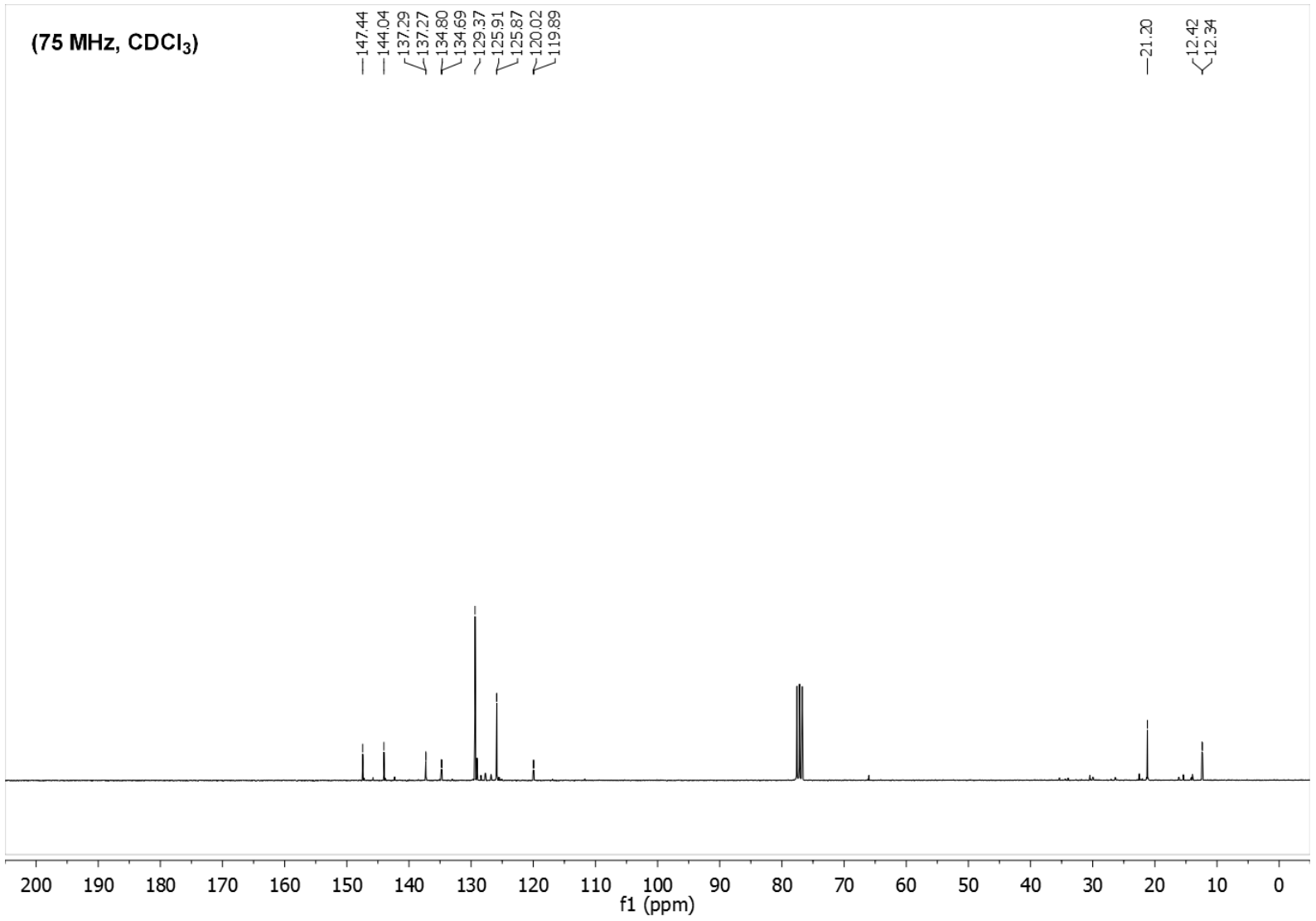

(282 MHz, $\mathrm{CDCl}_{3}$ )

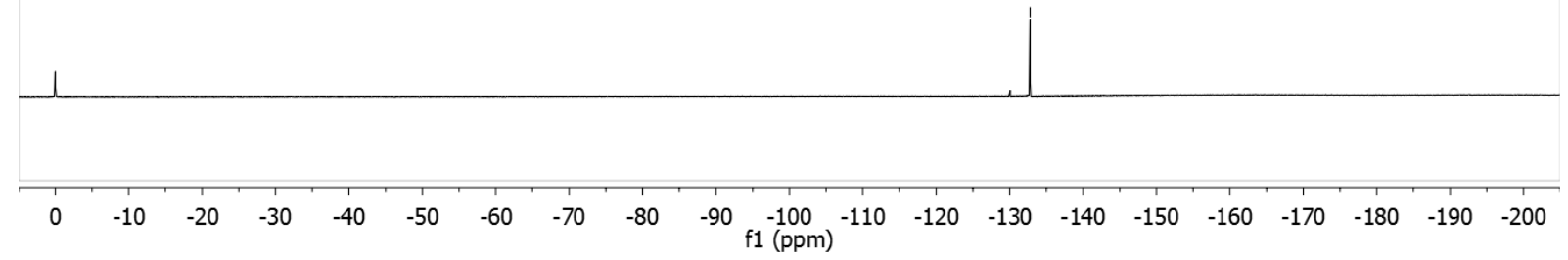




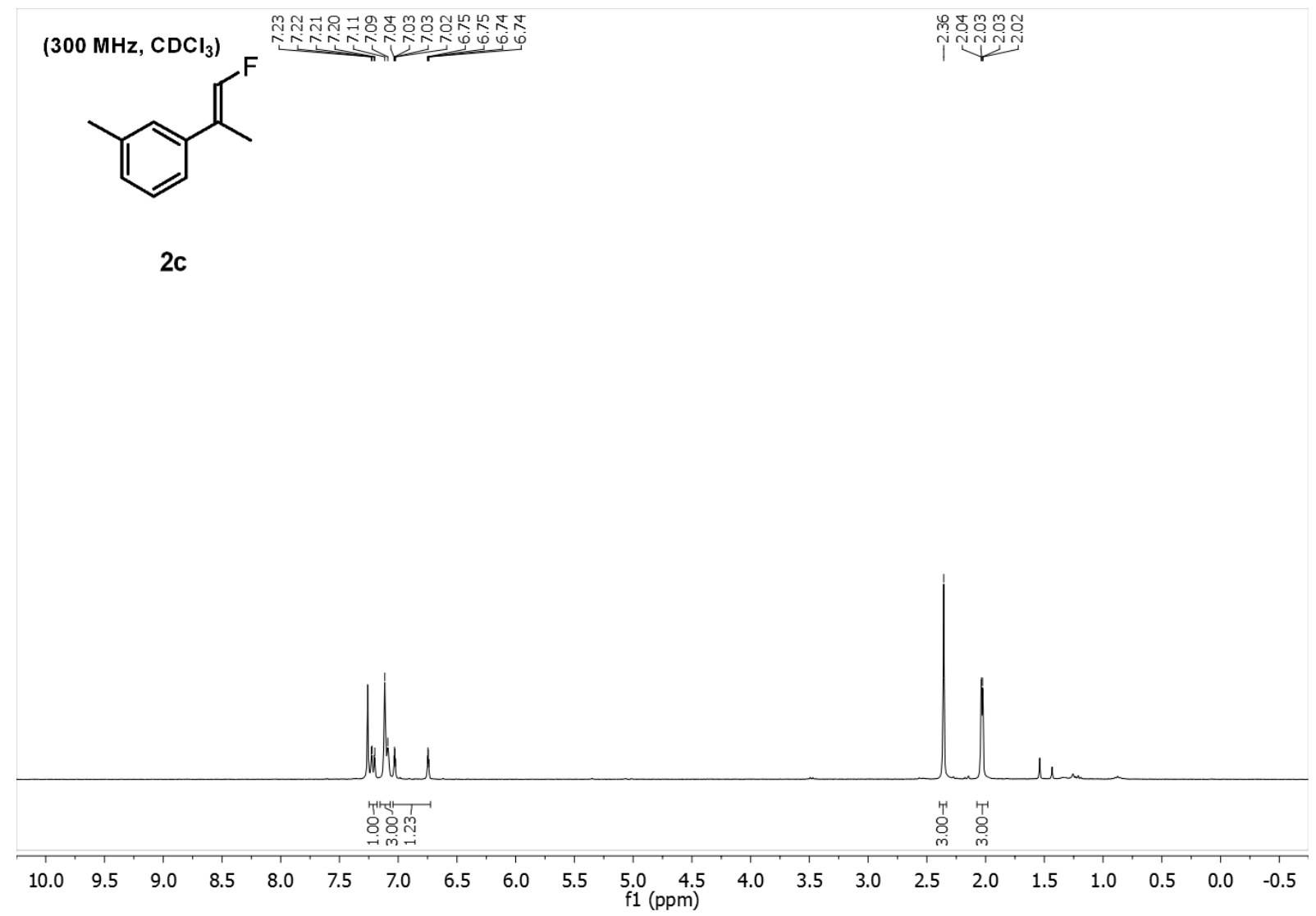

(75 MHz, $\mathrm{CDCl}_{3}$ )

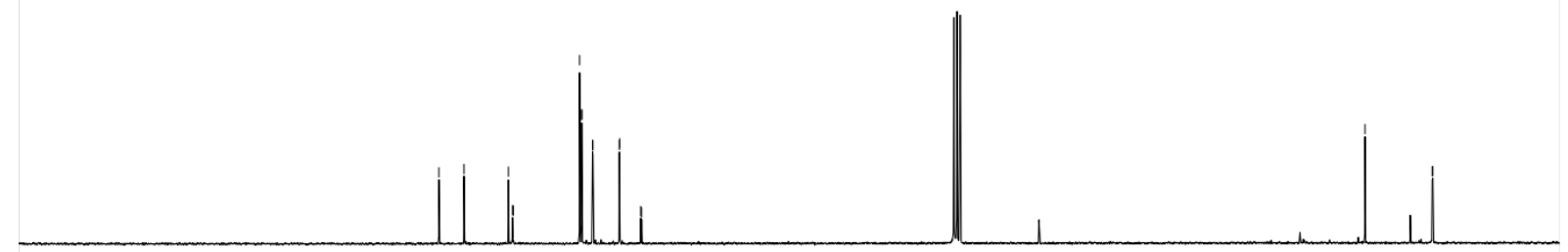


(282 MHz, $\mathrm{CDCl}_{3}$ )
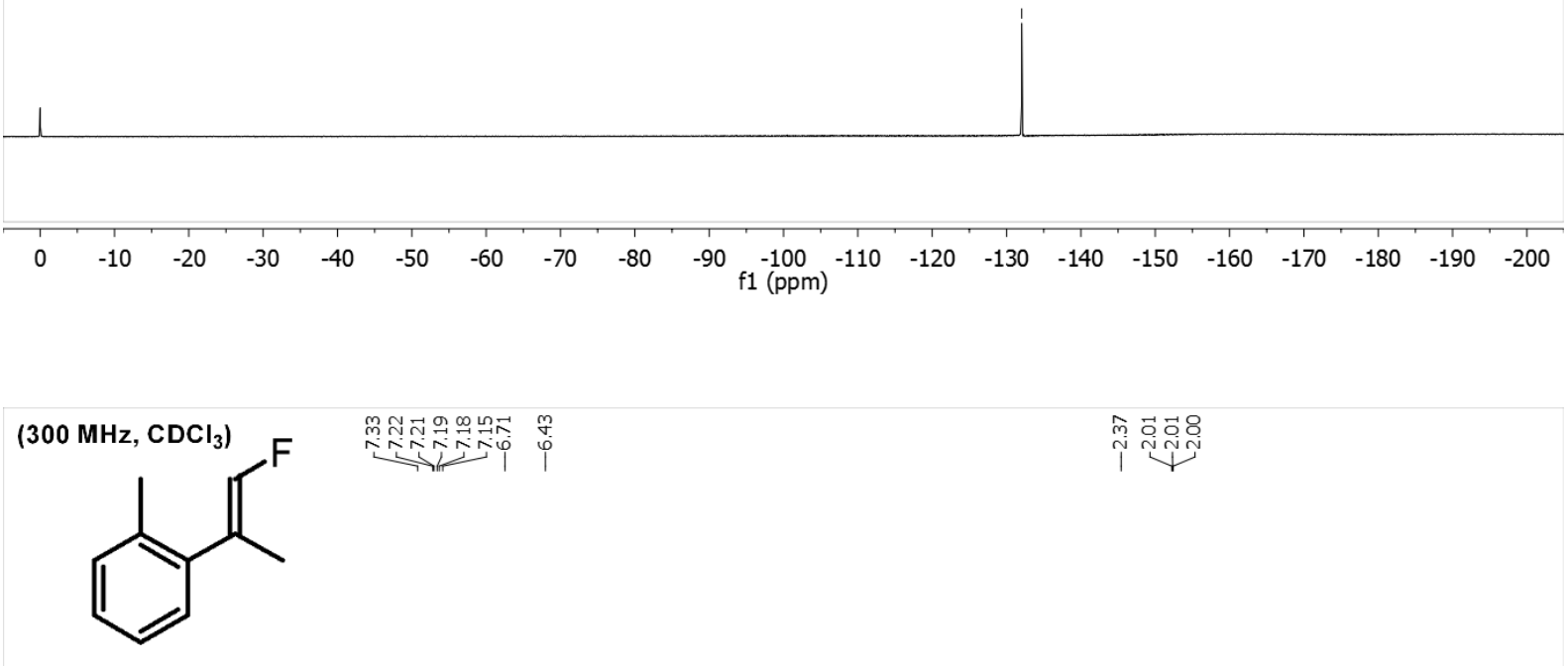

2d

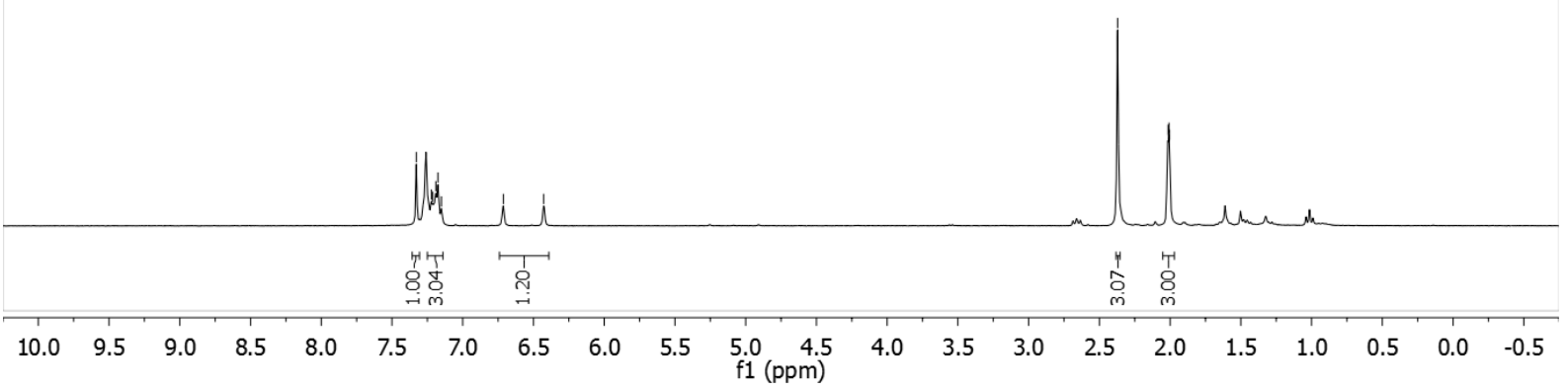


$\left(75 \mathrm{MHz}, \mathrm{CDCl}_{3}\right)$

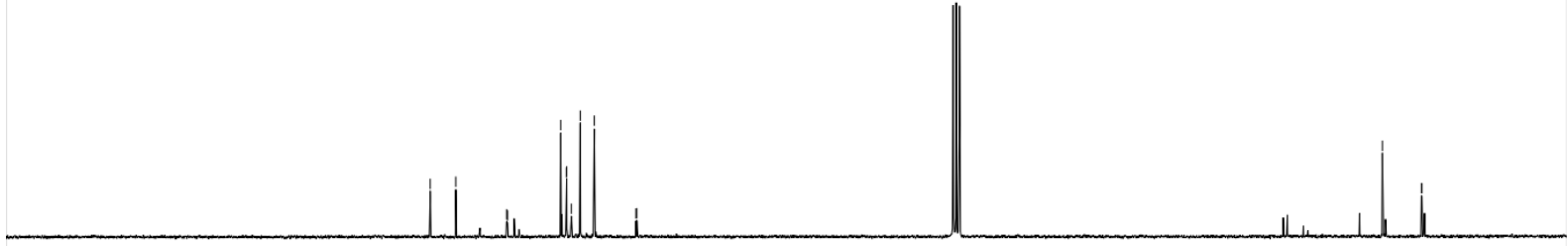

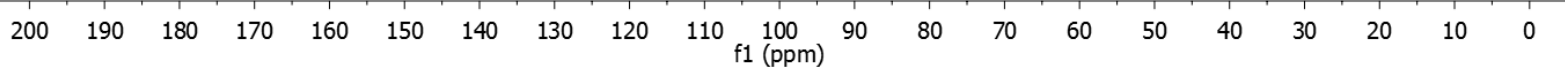

$\left(282 \mathrm{MHz}, \mathrm{CDCl}_{3}\right)$

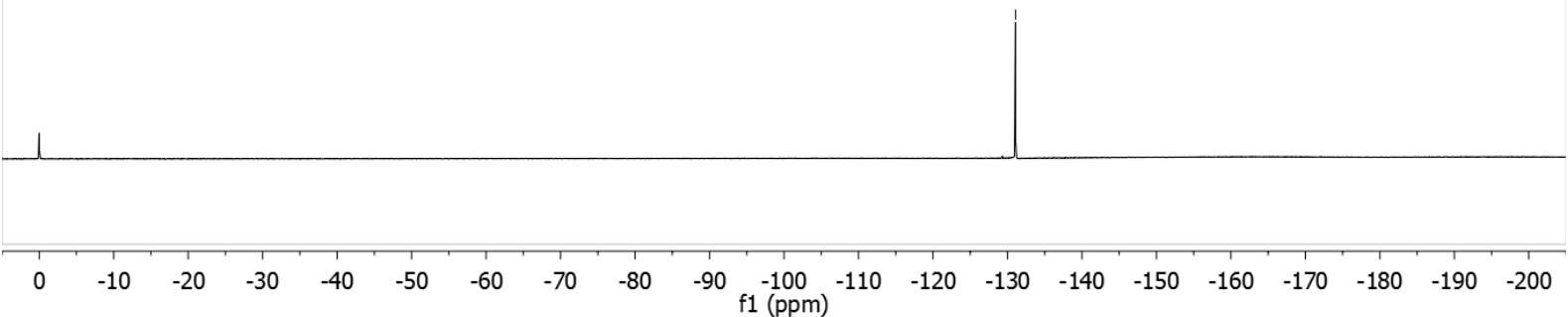




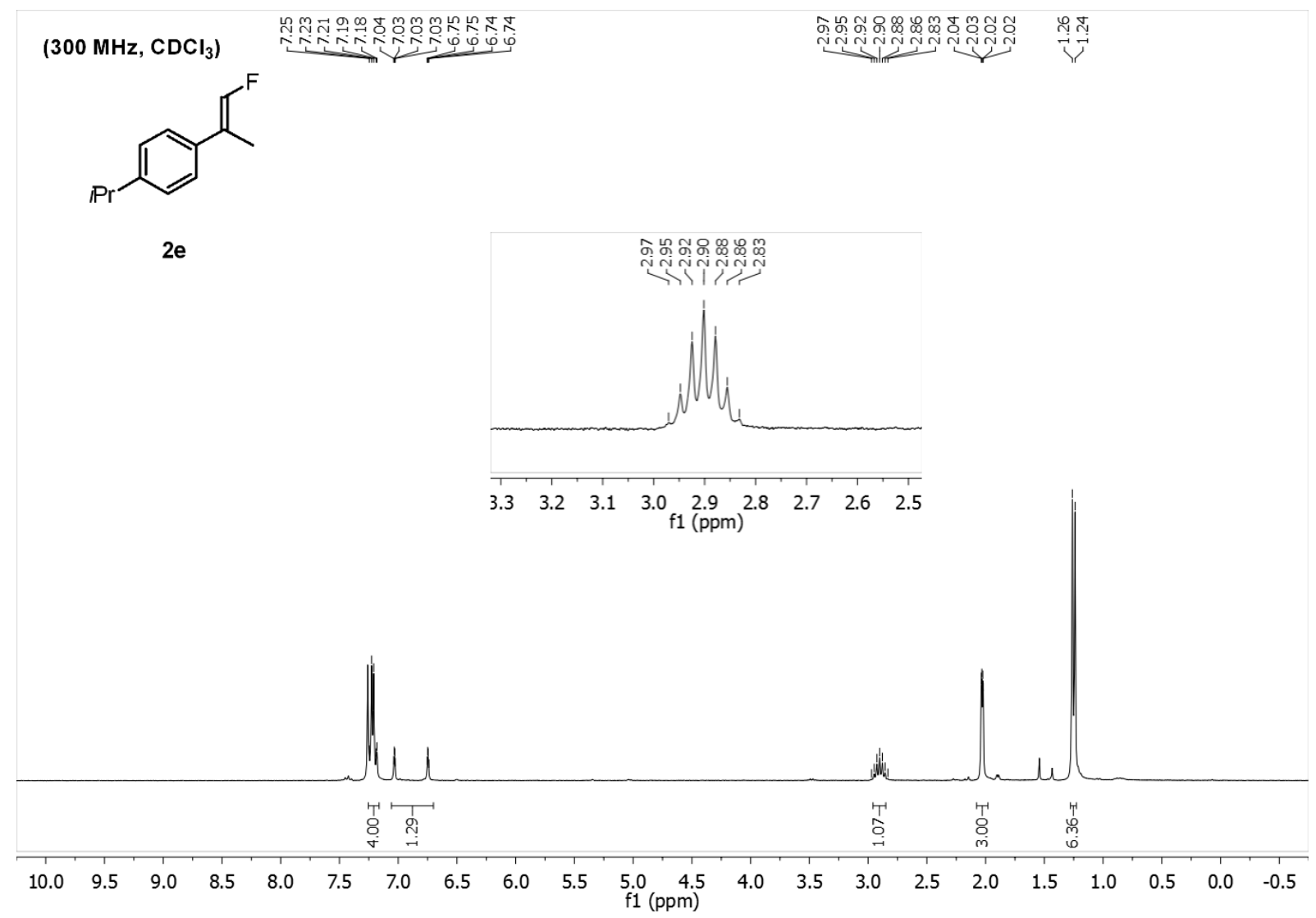

(75 MHz, $\mathrm{CDCl}_{3}$ )
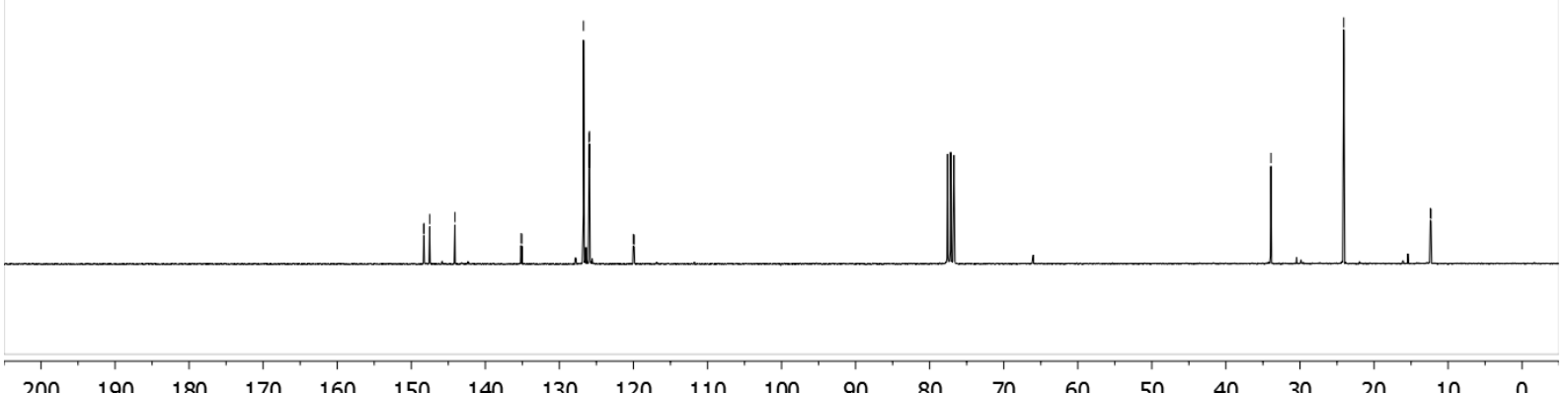
(282 MHz, $\mathrm{CDCl}_{3}$ )
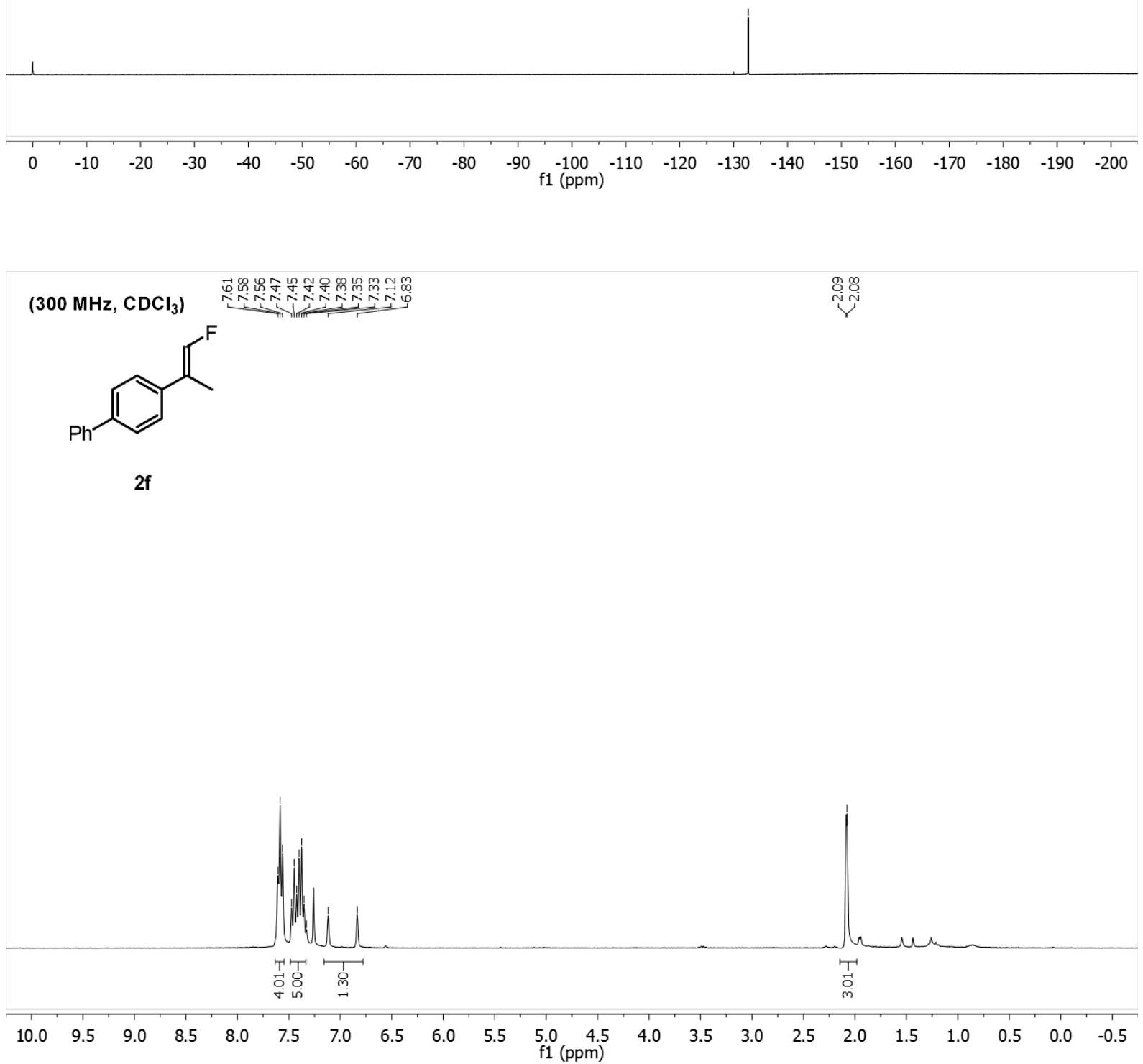


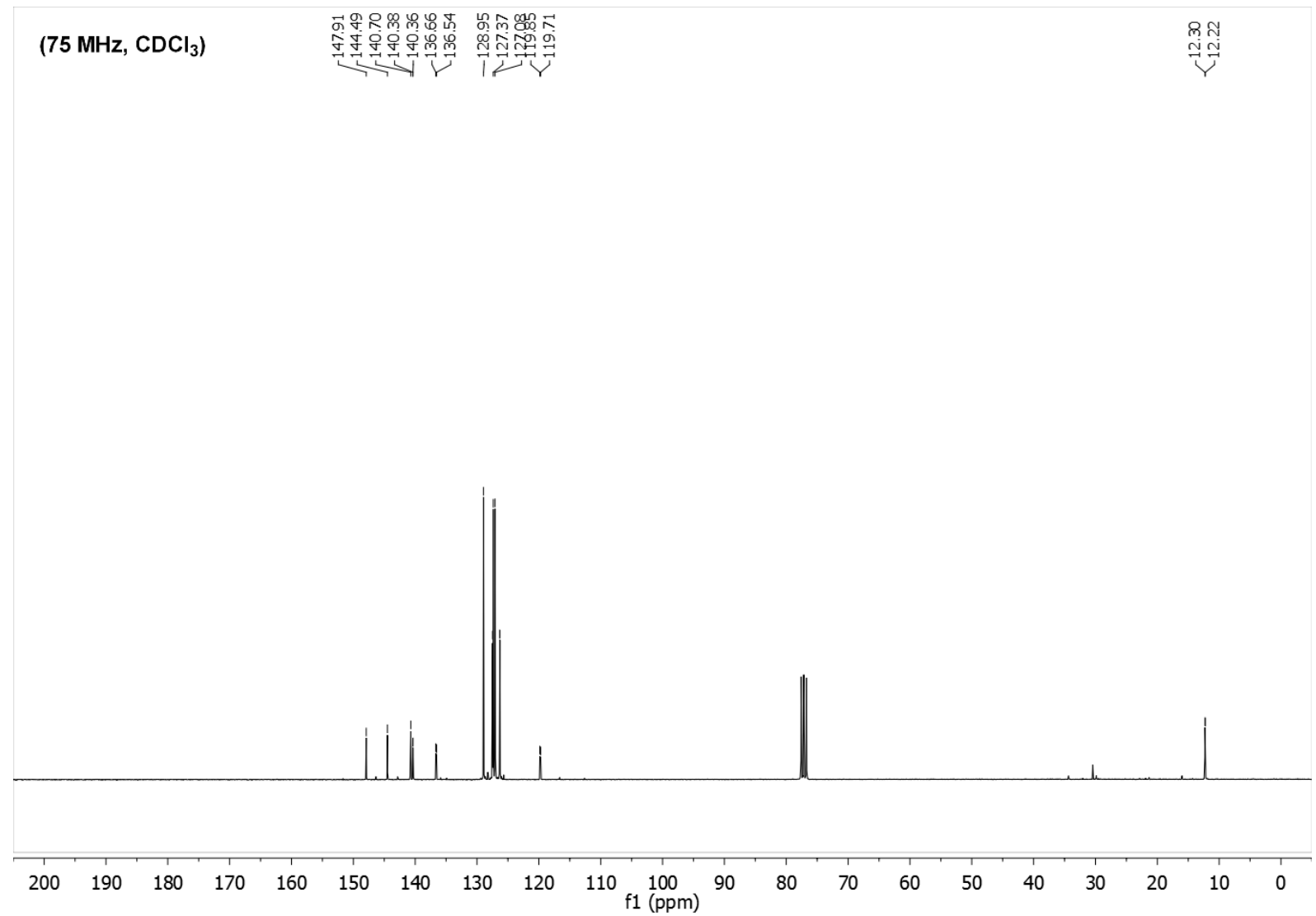

(282 $\mathrm{MHz}, \mathrm{CDCl}_{3}$ ) 


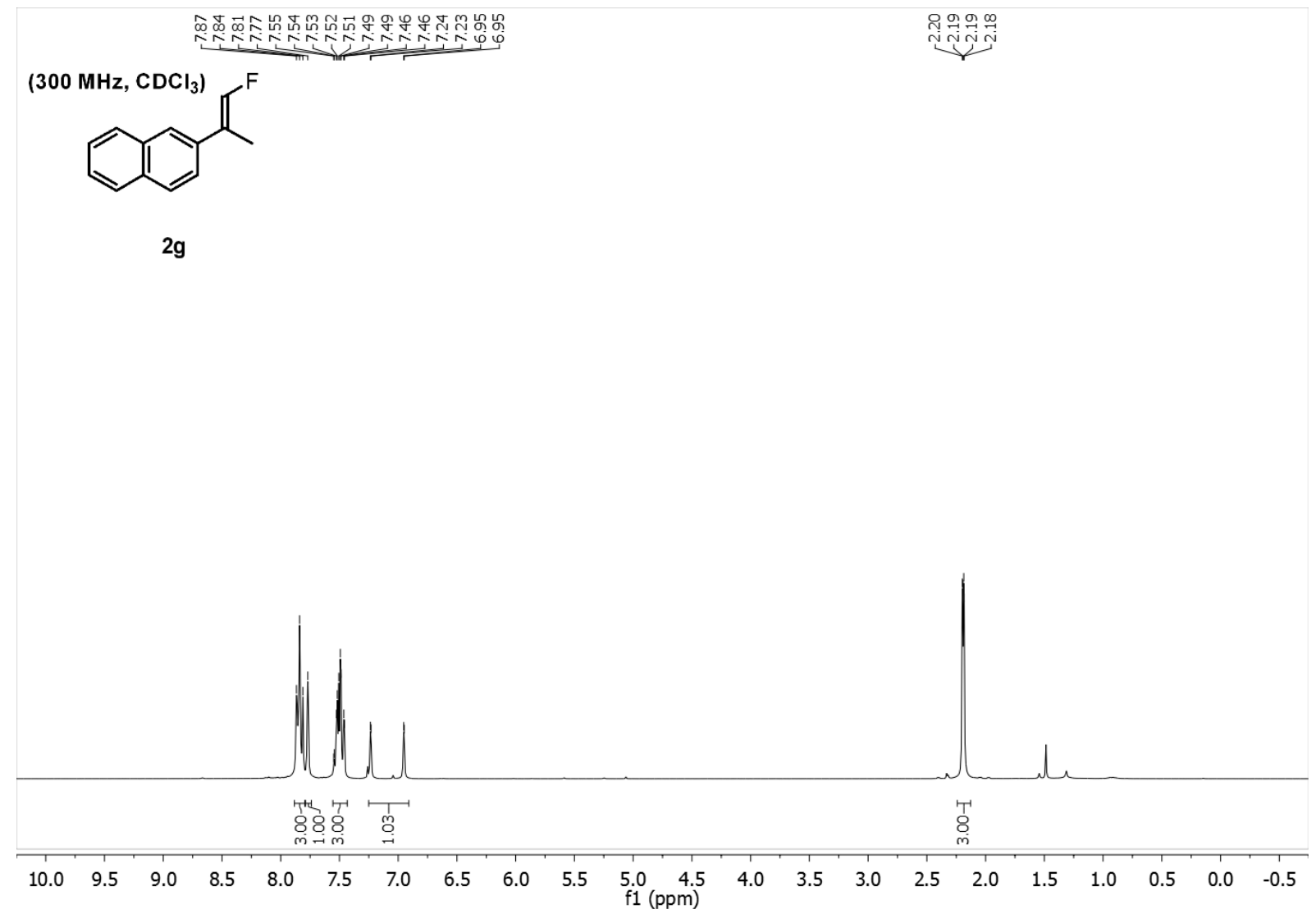

(75 $\mathrm{MHz}, \mathrm{CDCl}_{3}$ )

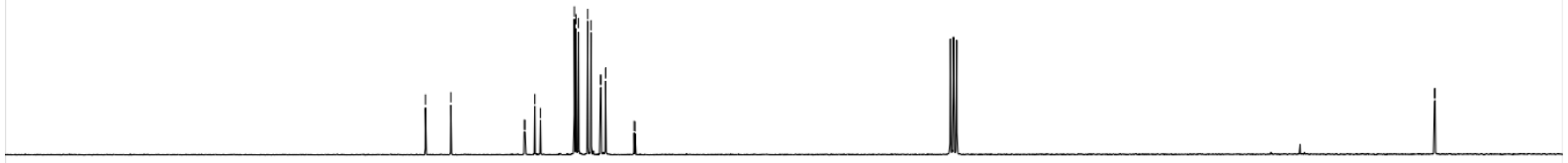


(282 $\mathrm{MHz}, \mathrm{CDCl}_{3}$ )

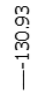

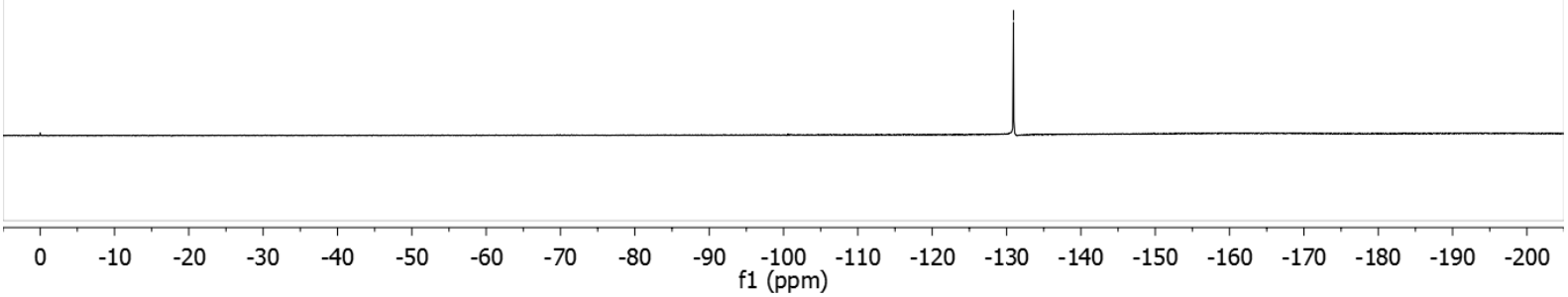

$\left(300 \mathrm{MHz}, \mathrm{CDCl}_{3}\right) \underbrace{4}$

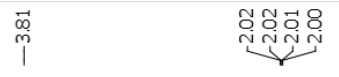<smiles>COc1ccc(/C(C)=C/F)cc1</smiles>

2h

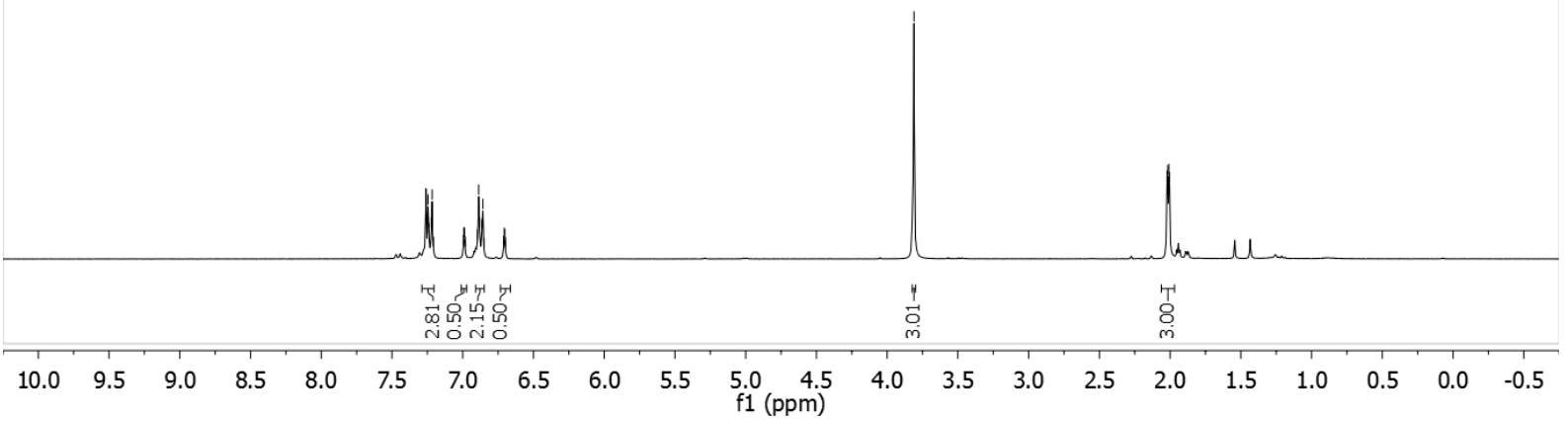




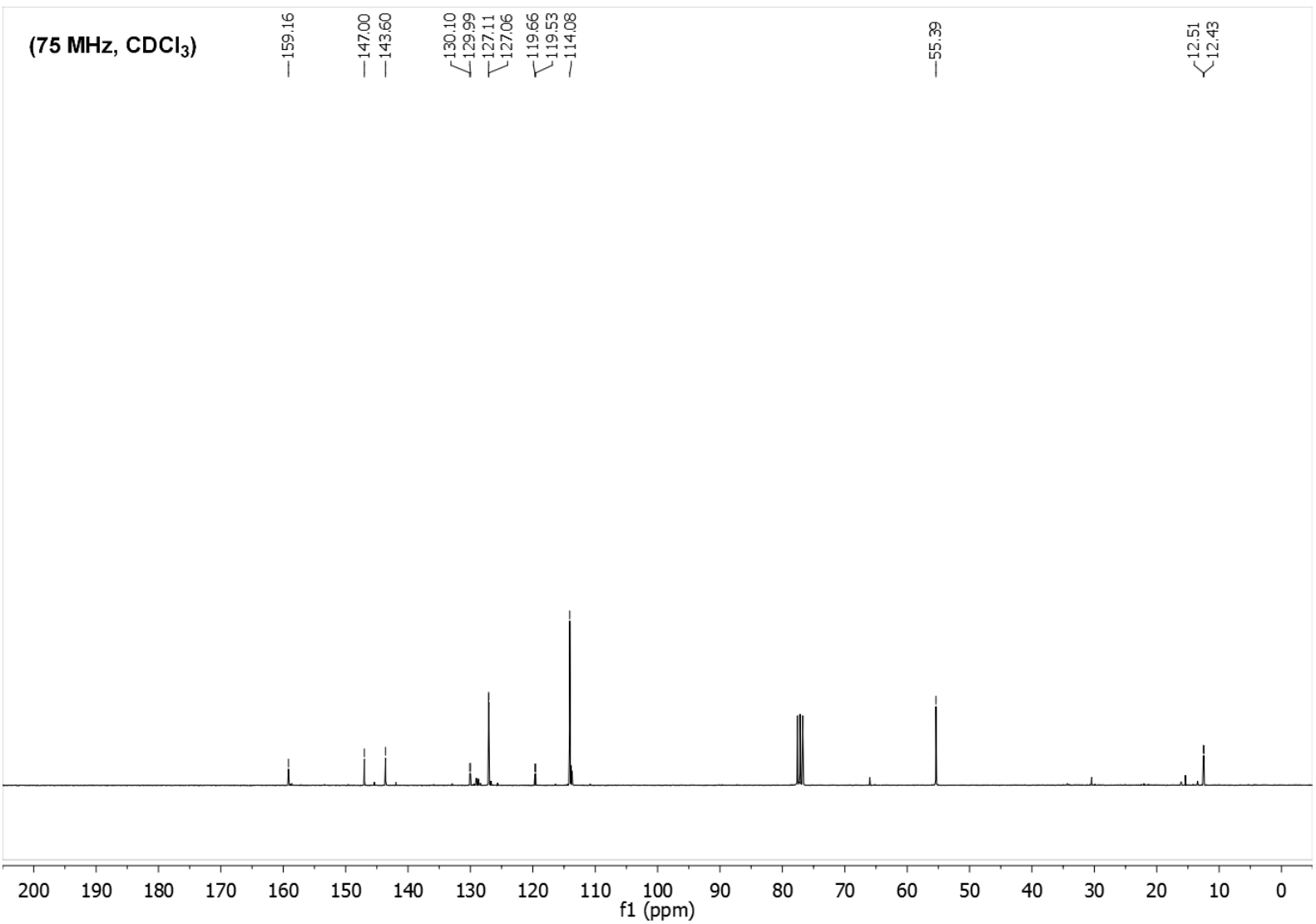

(282 $\mathrm{MHz}, \mathrm{CDCl}_{3}$ )

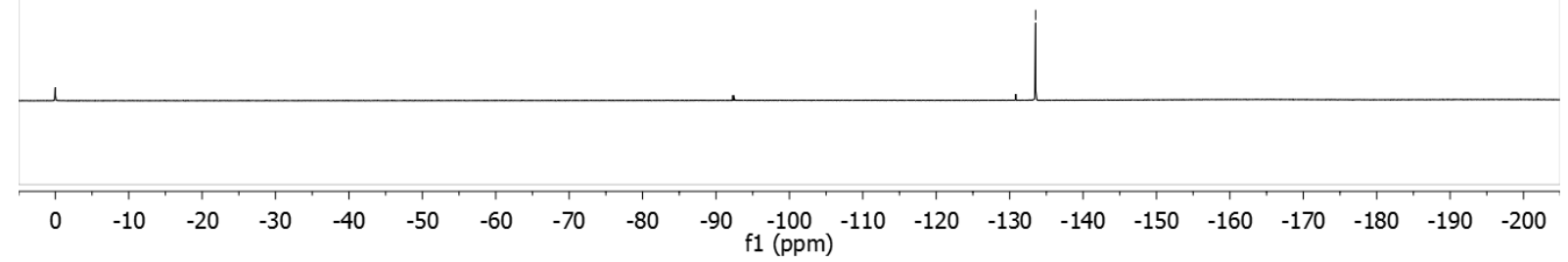




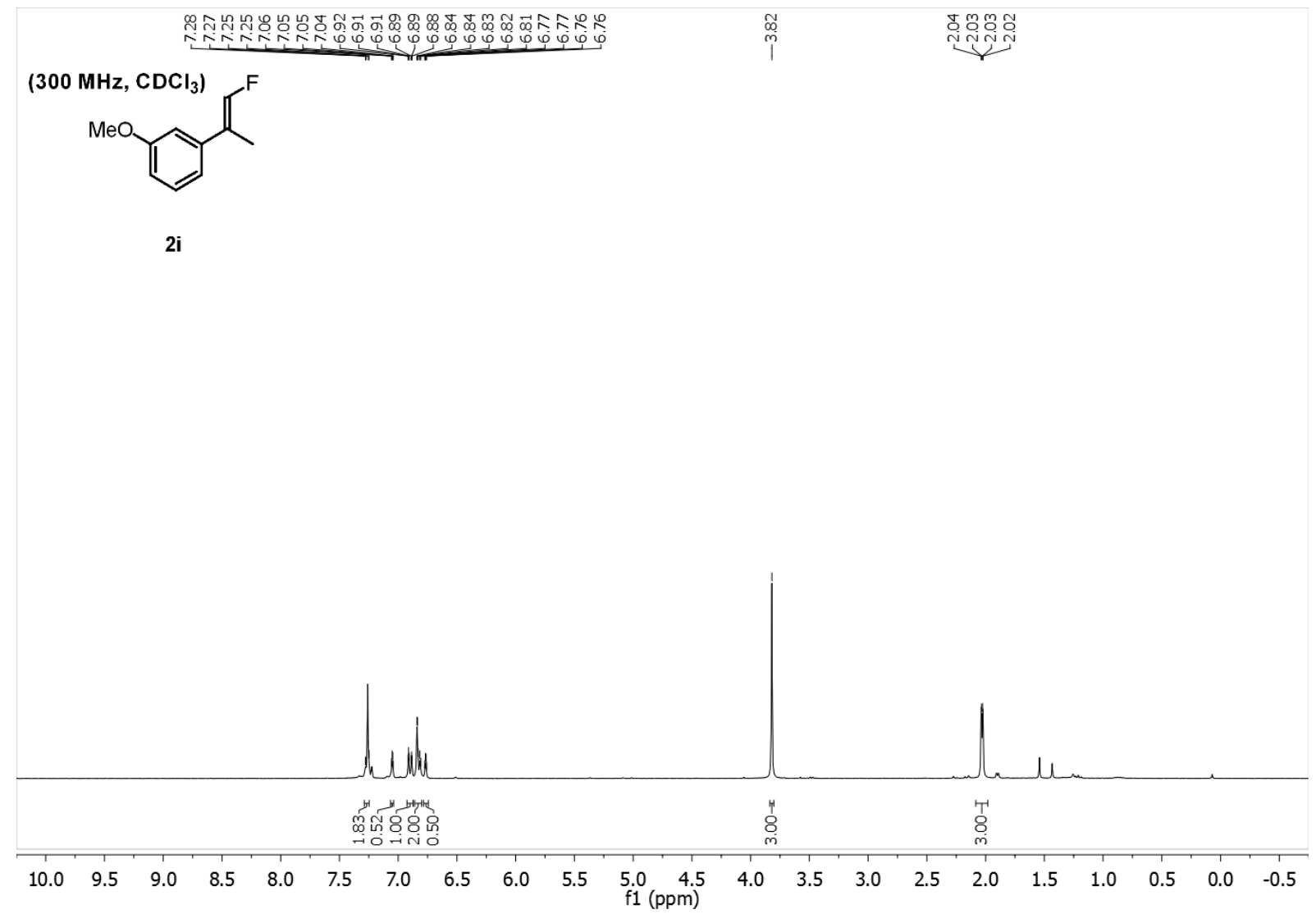

(75 MHz, $\left.\mathrm{CDCl}_{3}\right)$ 舀
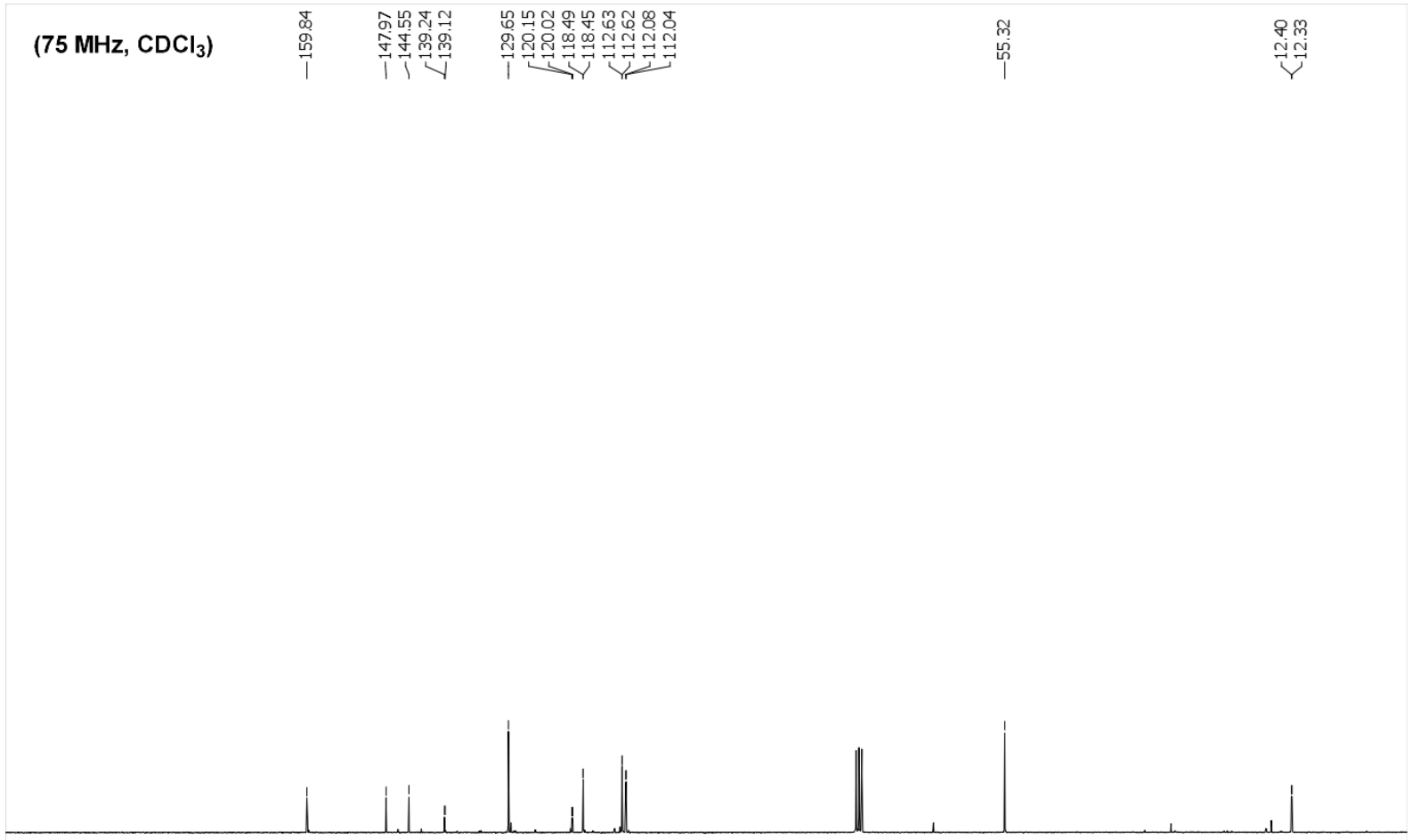

$\begin{array}{lllllllllllllllllllllll}200 & 190 & 180 & 170 & 160 & 150 & 140 & 130 & 120 & 110 & 100 & 90 & 80 & 70 & 60 & 50 & 40 & 30 & 20 & 10 & 0\end{array}$ 
(282 MHz, $\mathrm{CDCl}_{3}$ )

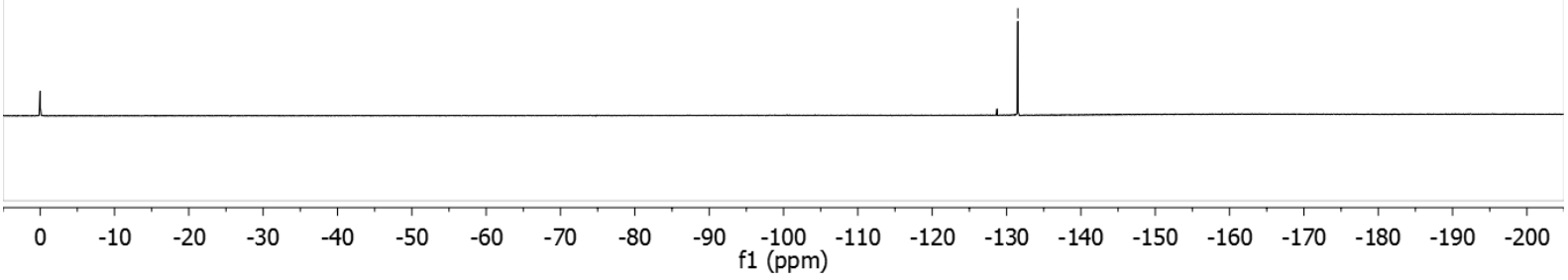

(300 MHz, $\mathrm{CDCl}_{3}$ )<smiles>C/C(=C\F)c1ccc(N(C)C)cc1</smiles>

กั

2j

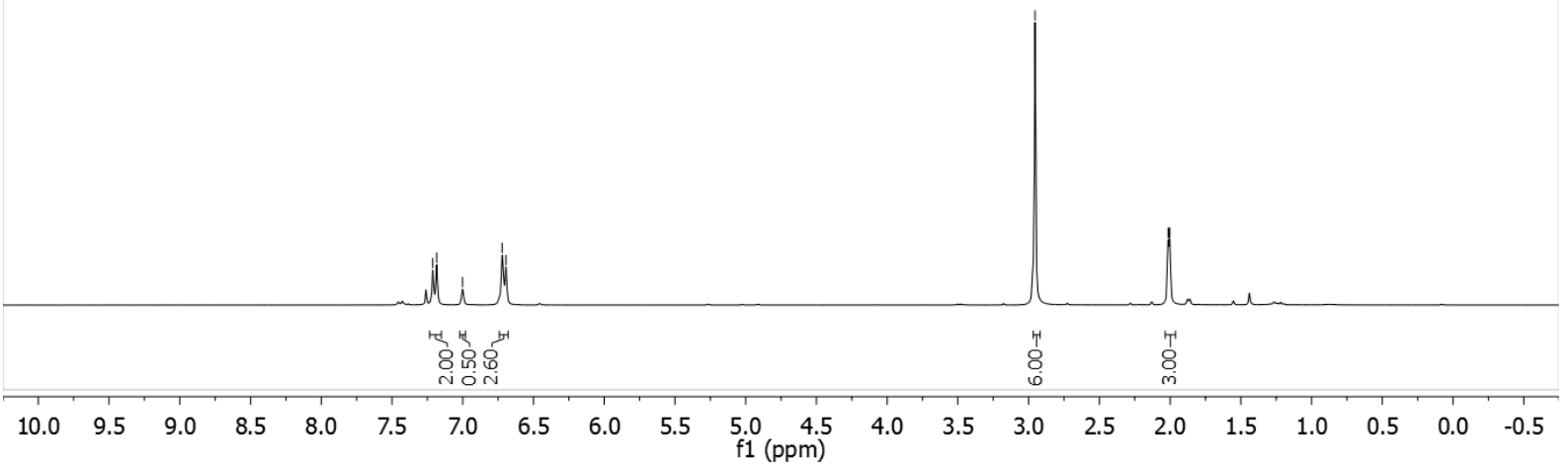




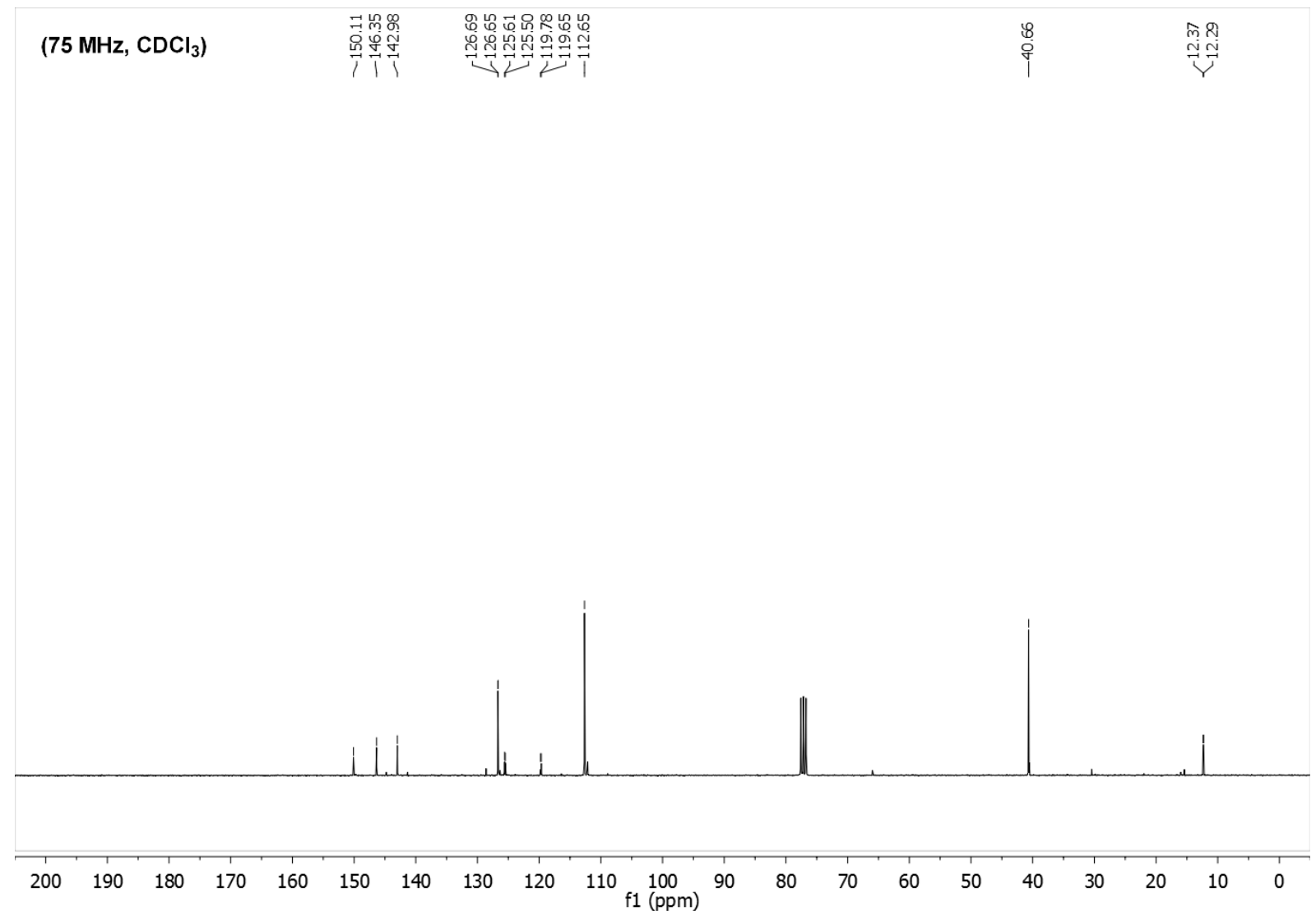

(282 $\mathrm{MHz}, \mathrm{CDCl}_{3}$ )

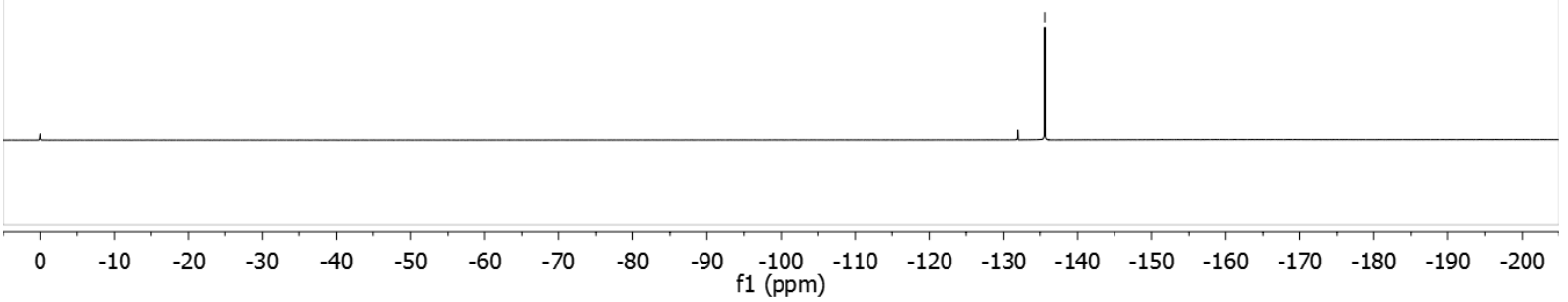




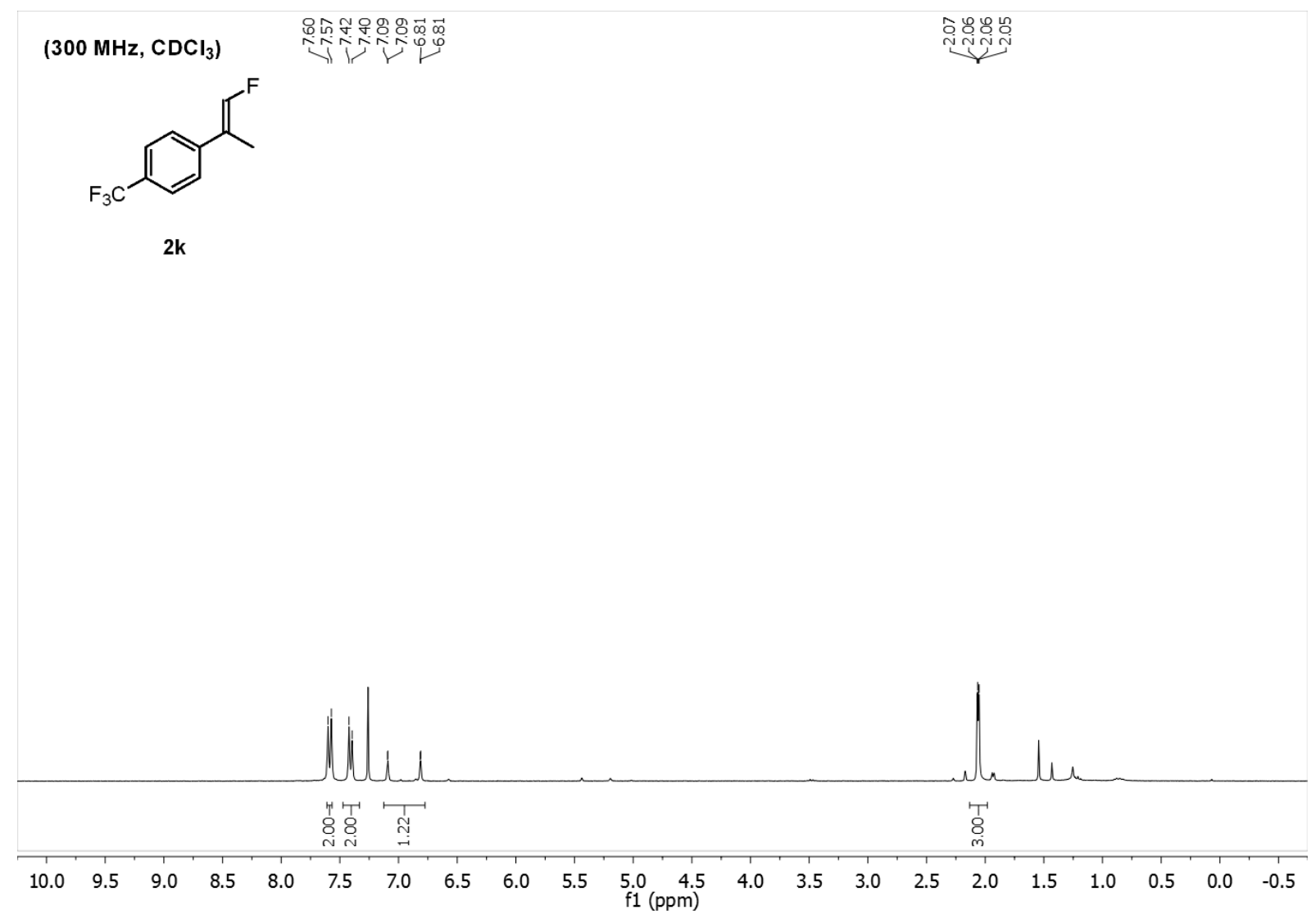

(75 $\mathrm{MHz}, \mathrm{CDCl}_{3}$ )

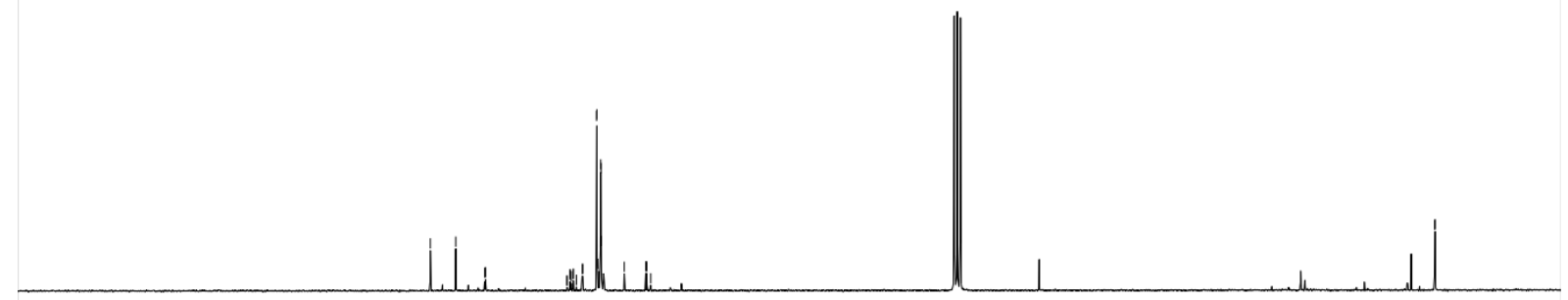


(282 MHz, $\mathrm{CDCl}_{3}$ )
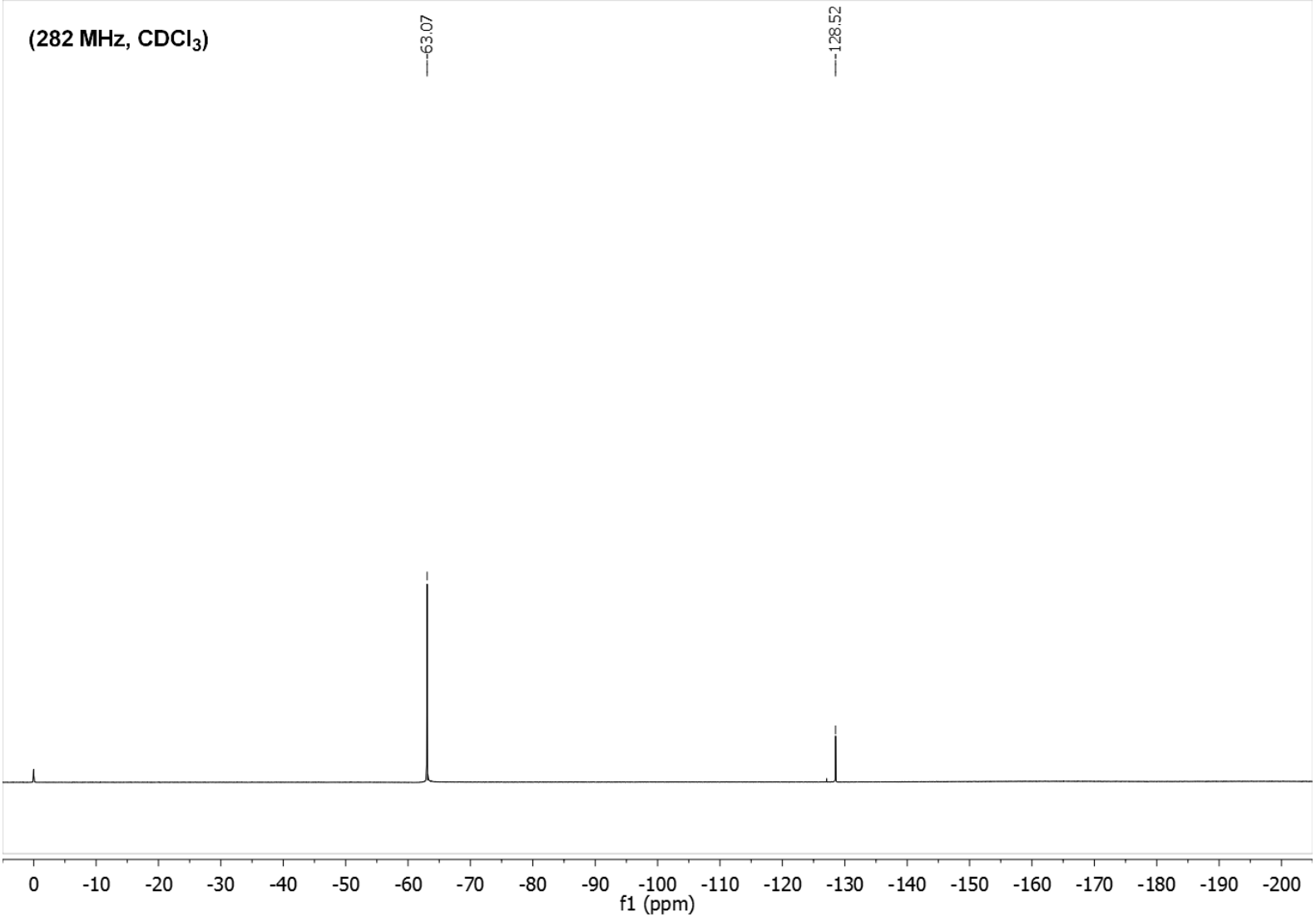

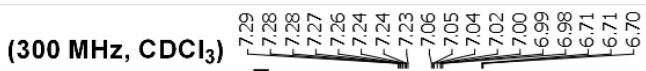<smiles>CC(=CF)c1ccc(F)cc1</smiles>

2I

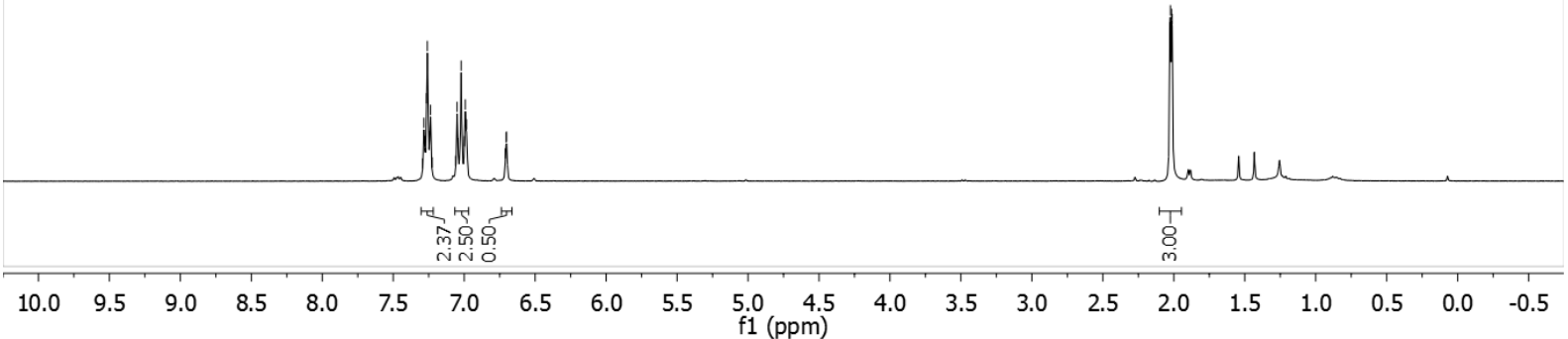




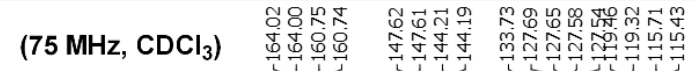

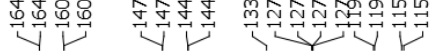

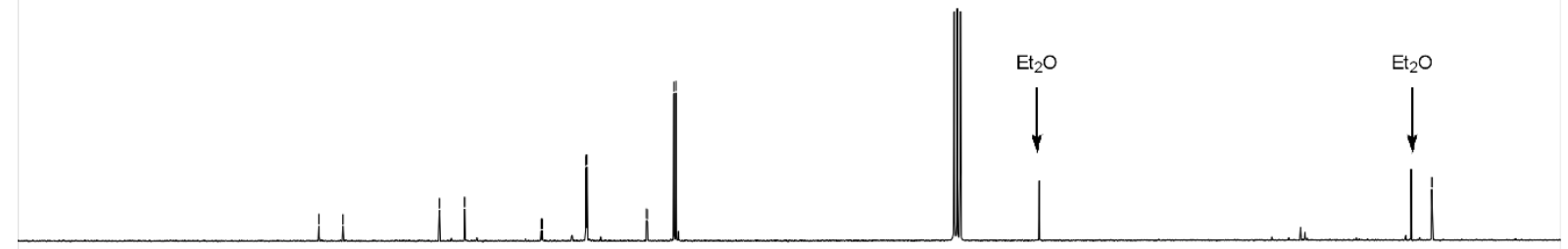

$\begin{array}{lllllllllllllllllllllllllllll}200 & 190 & 180 & 170 & 160 & 150 & 140 & 130 & 120 & 110 & 100 & 90 & 80 & 70 & 60 & 50 & 40 & 30 & 20 & 10 & 0\end{array}$

$\left(282 \mathrm{MHz}, \mathrm{CDCl}_{3}\right)$

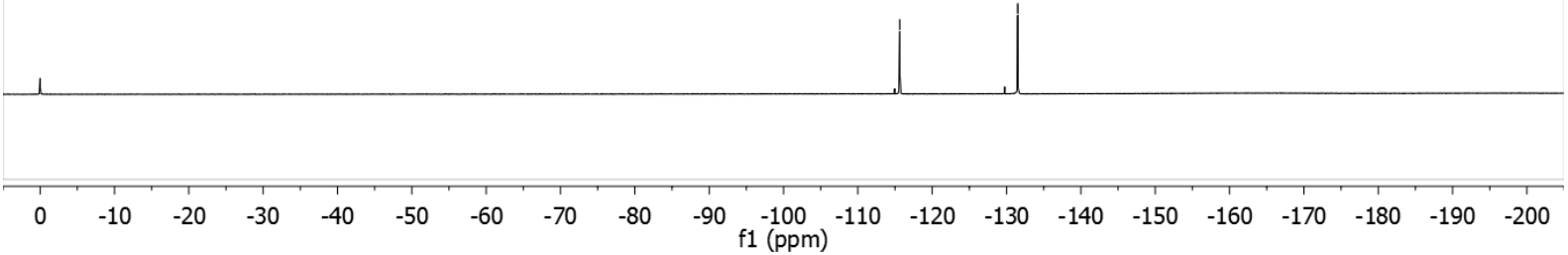




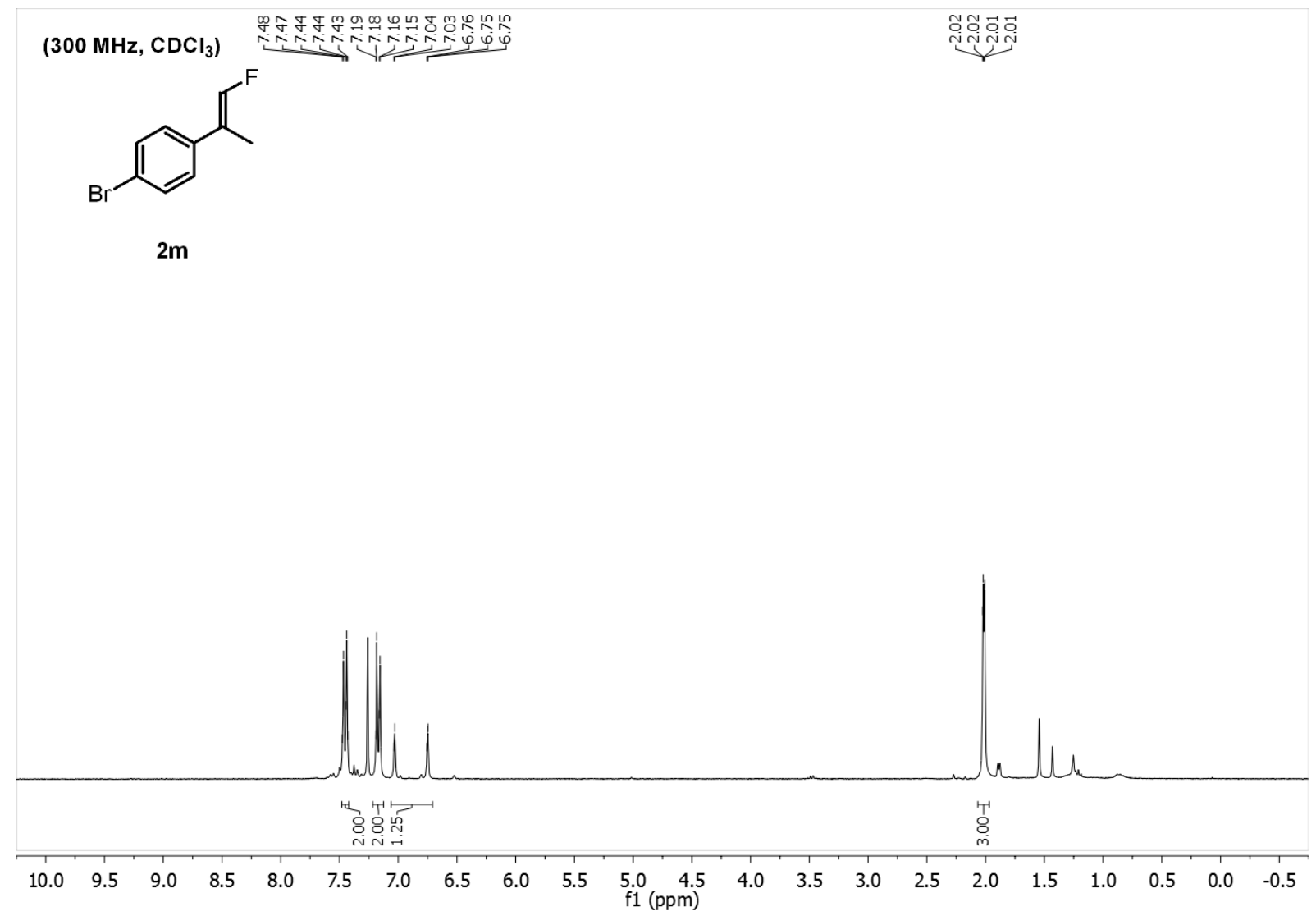

(75 MHz, $\mathrm{CDCl}_{3}$ )
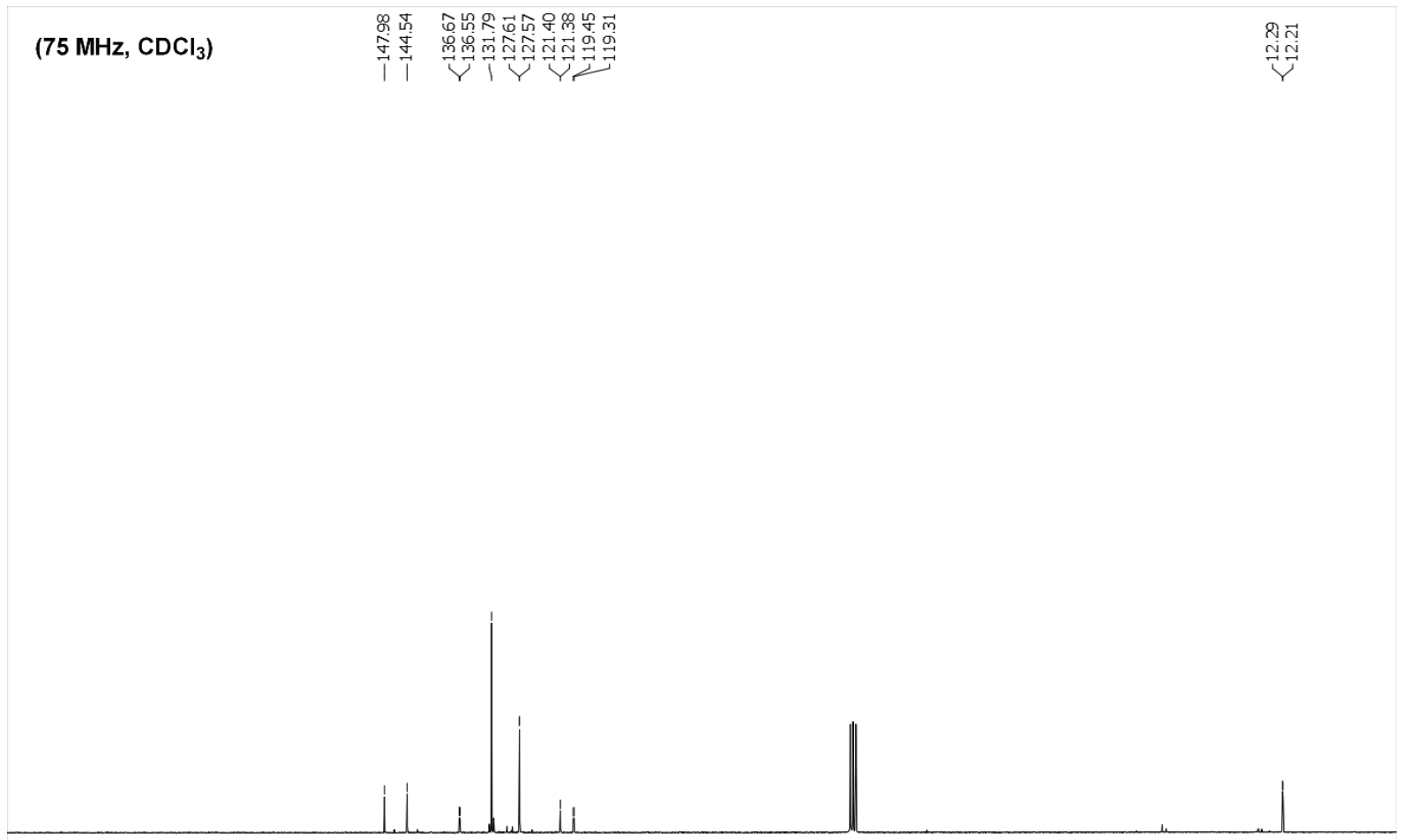
(282 MHz, $\mathrm{CDCl}_{3}$ )

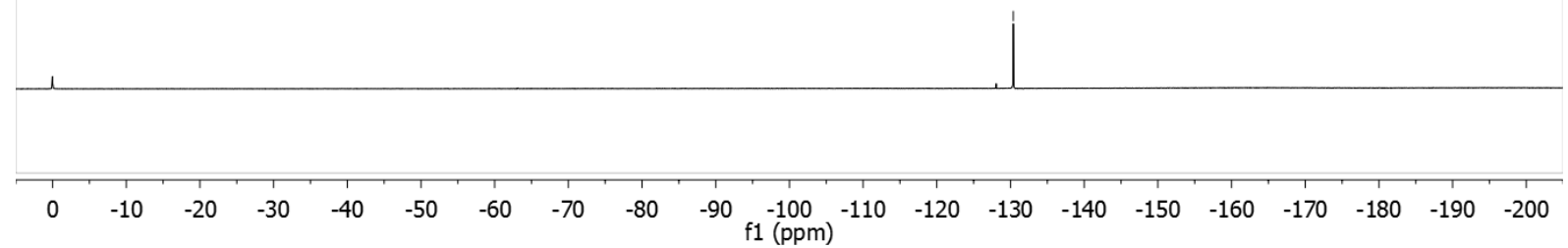

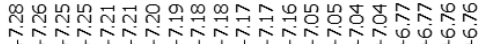

(300 MHz, $\mathrm{CDCl}_{3}$ )<smiles>CC(=CF)c1cccc(Cl)c1</smiles>

$2 n$

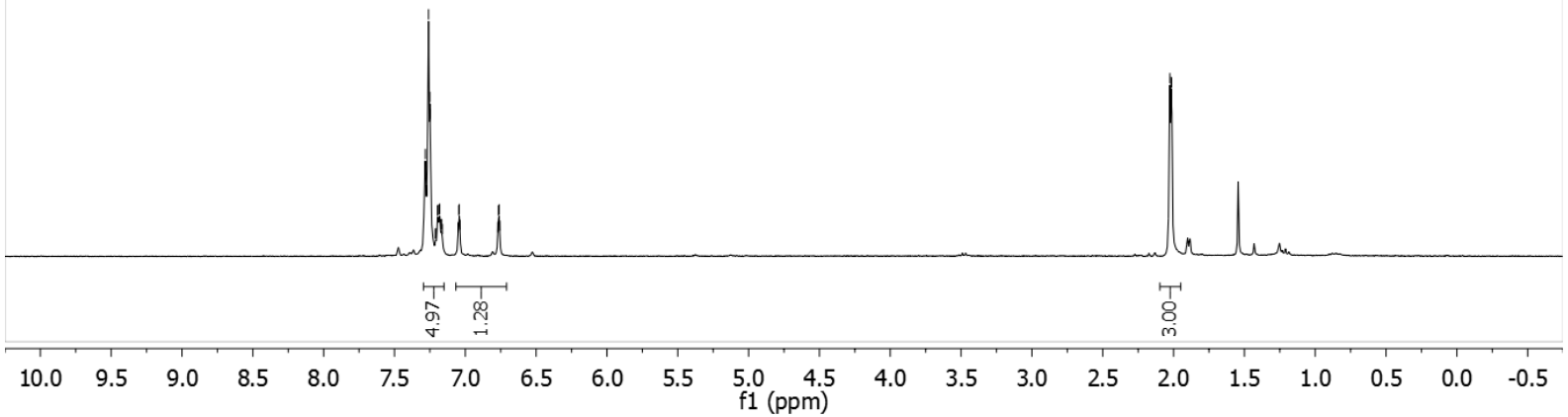




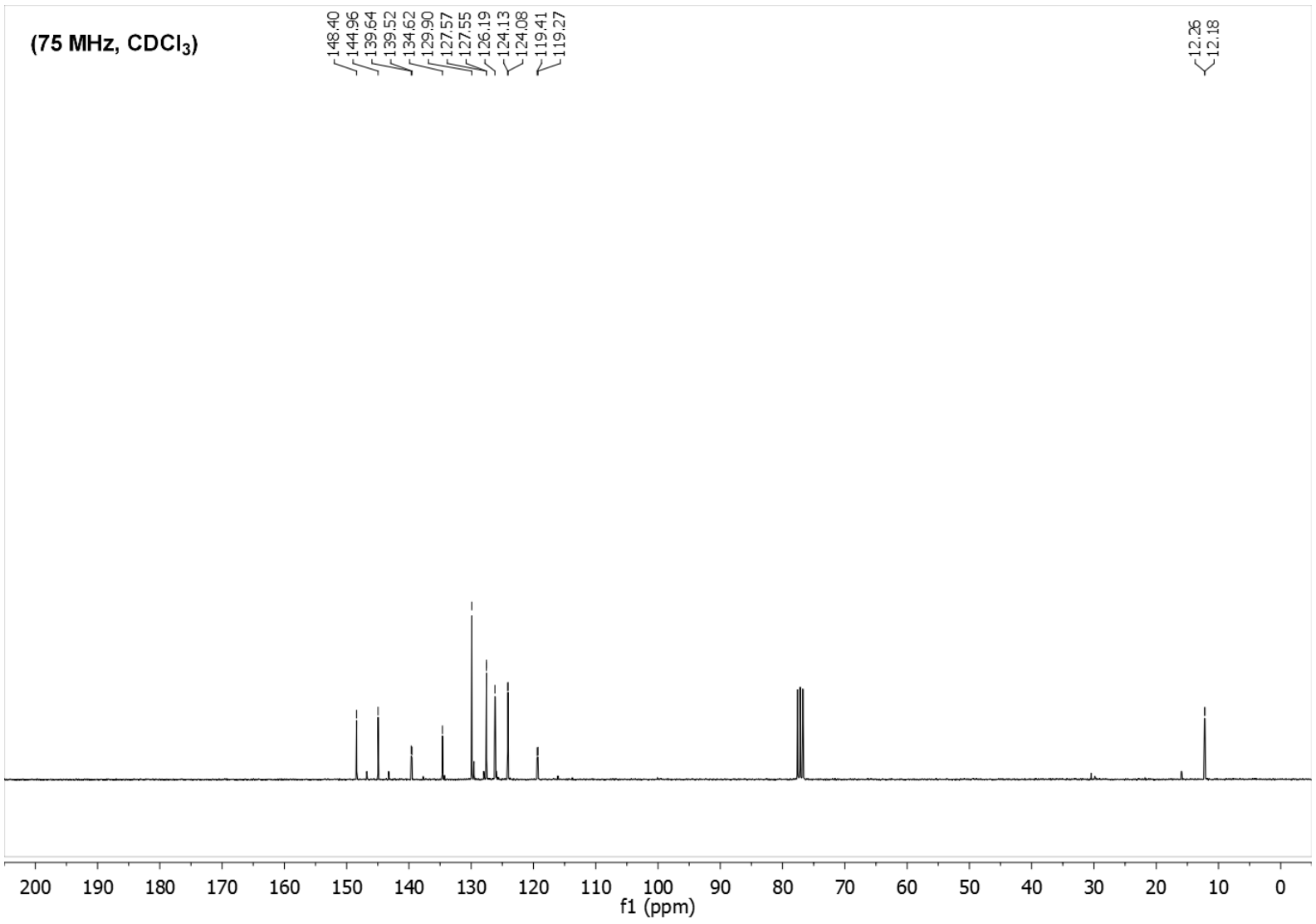

(282 MHz, $\mathrm{CDCl}_{3}$ )

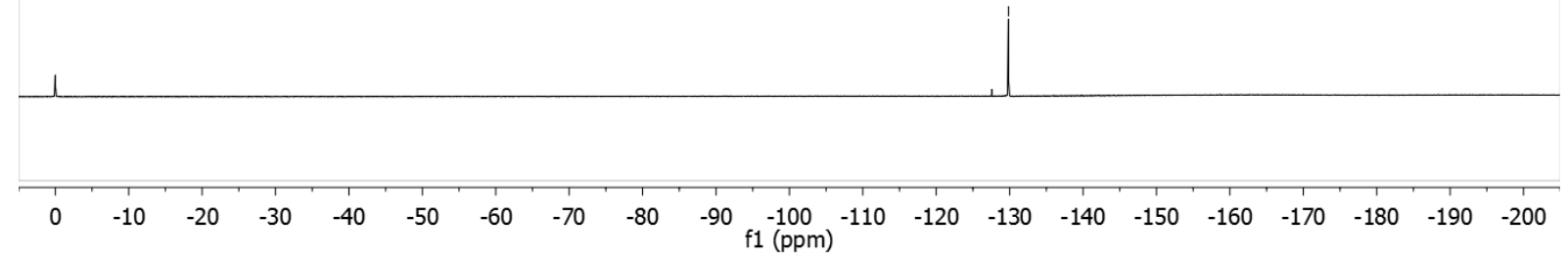



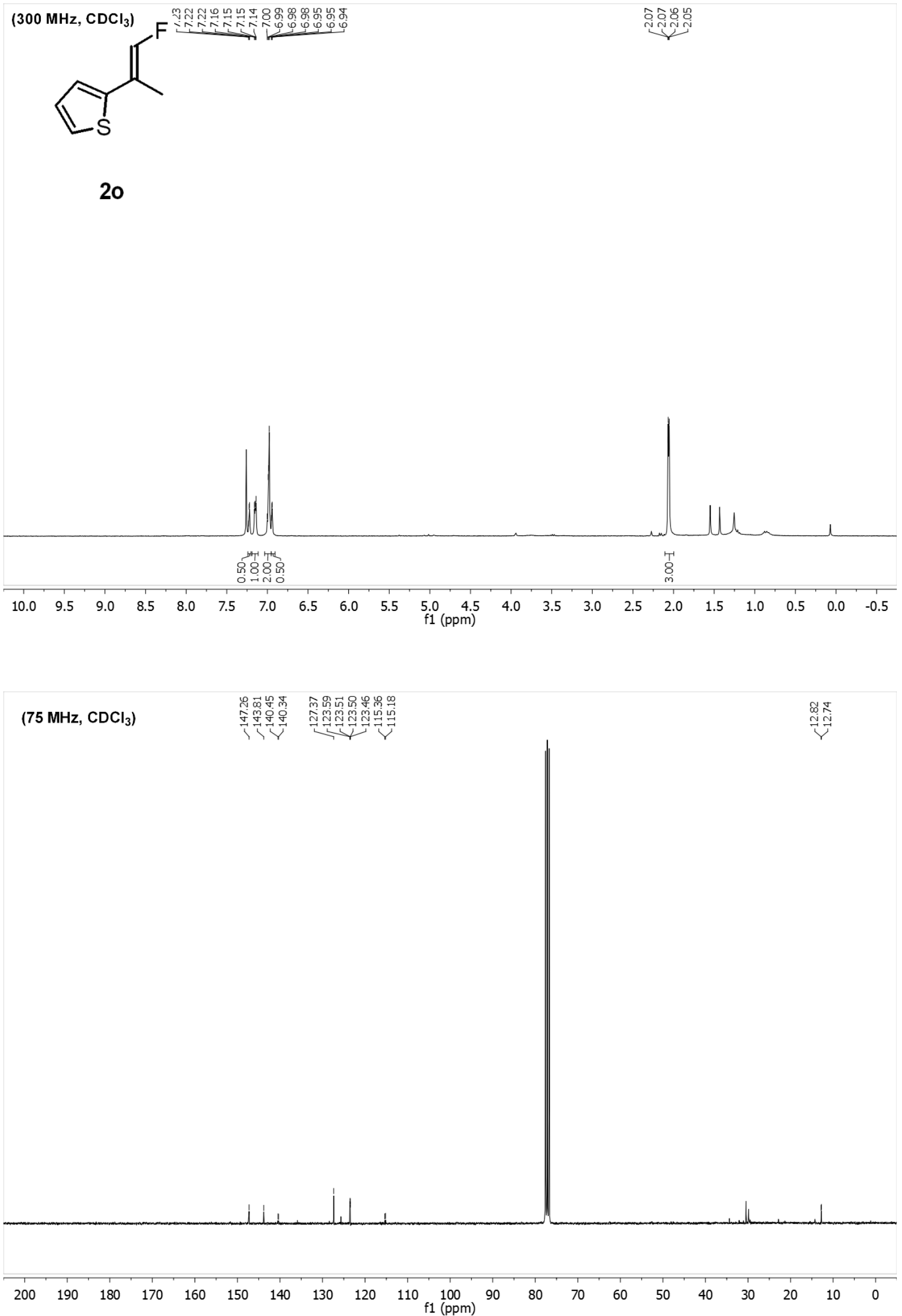
(282 MHz, $\mathrm{CDCl}_{3}$ )

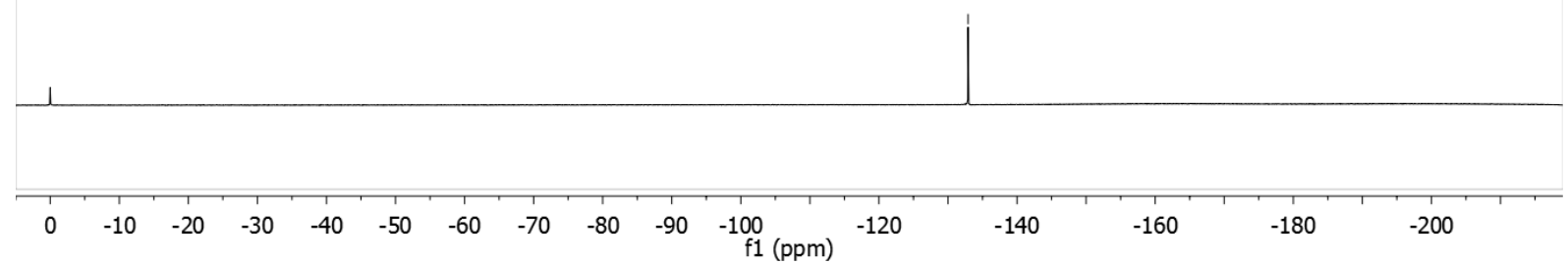

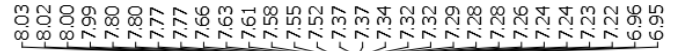<smiles>[B]n1cc(/C(C)=C/F)c2ccccc21</smiles>

$2 p$

(300 MHz, $\mathrm{CDCl}_{3}$ )

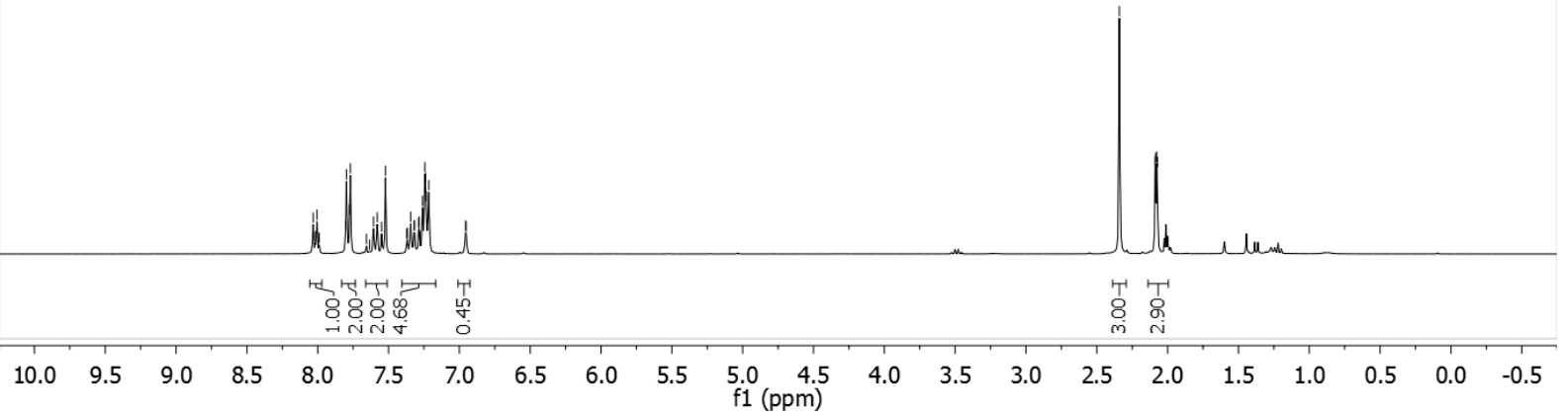




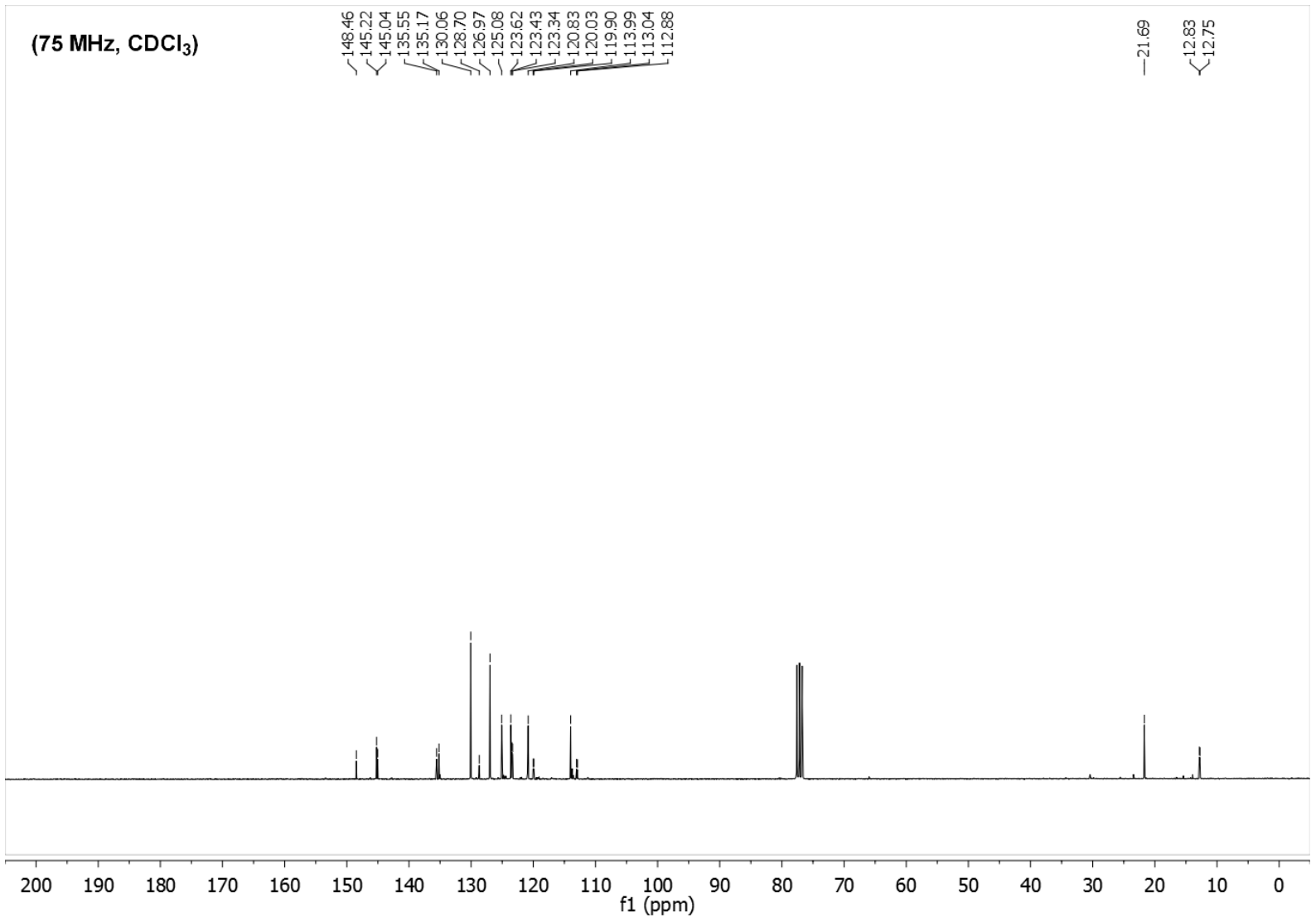

$\left(282 \mathrm{MHz} \mathrm{CDCl}_{3}\right)$

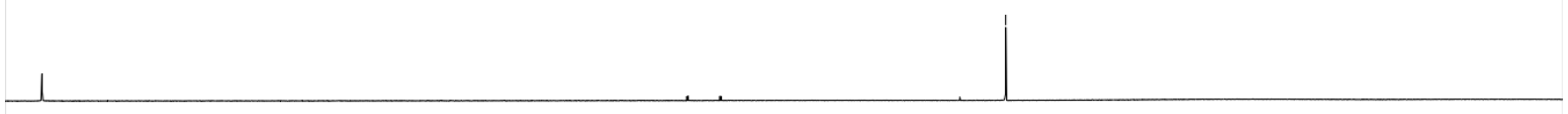

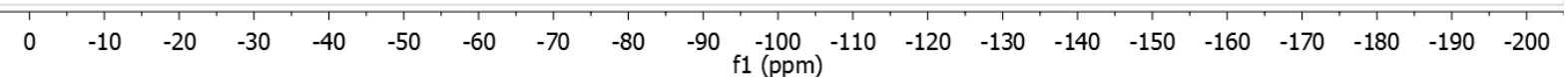




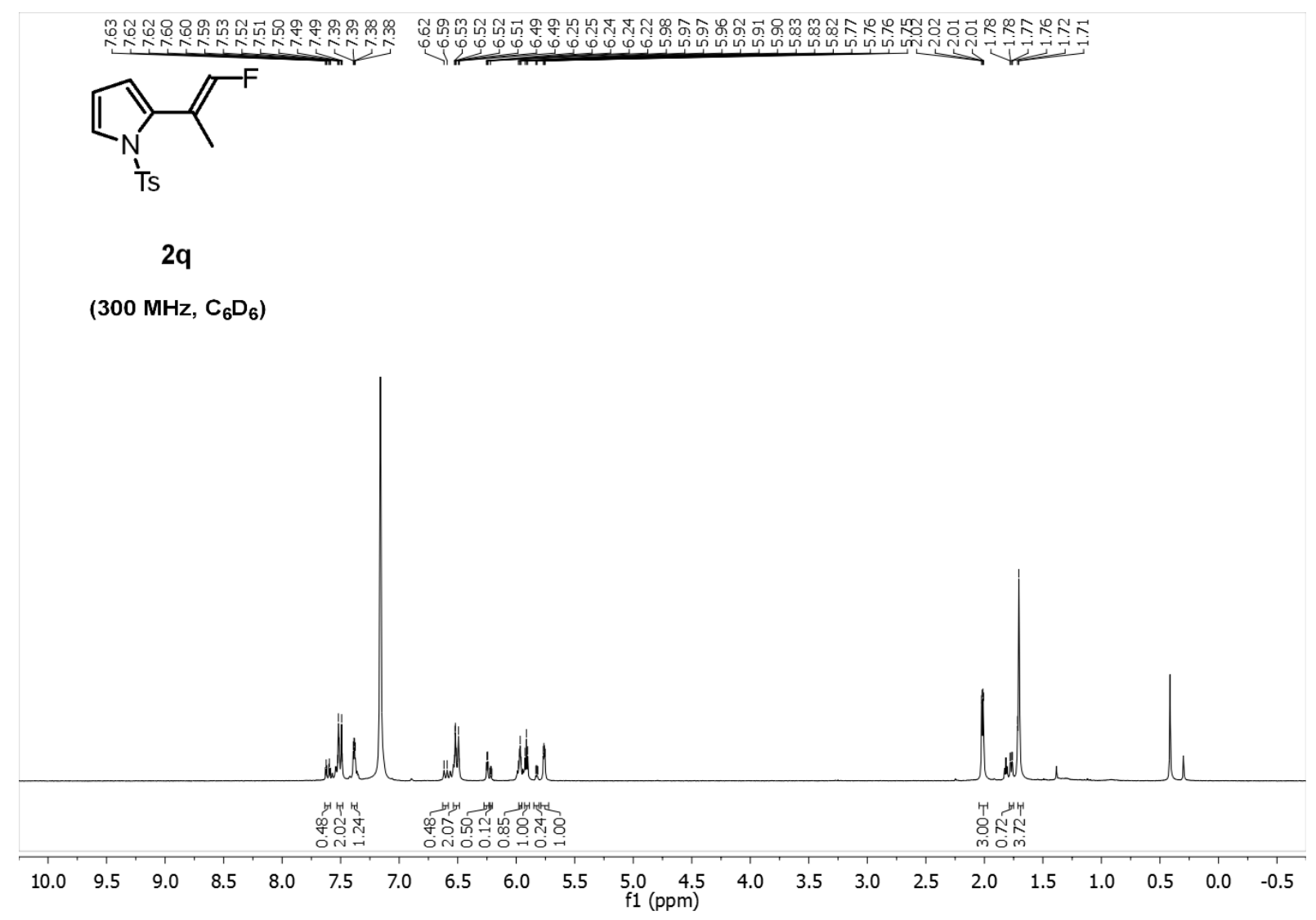

(75 MHz, $\left.\mathbf{C}_{6} \mathbf{D}_{6}\right) \quad$ P

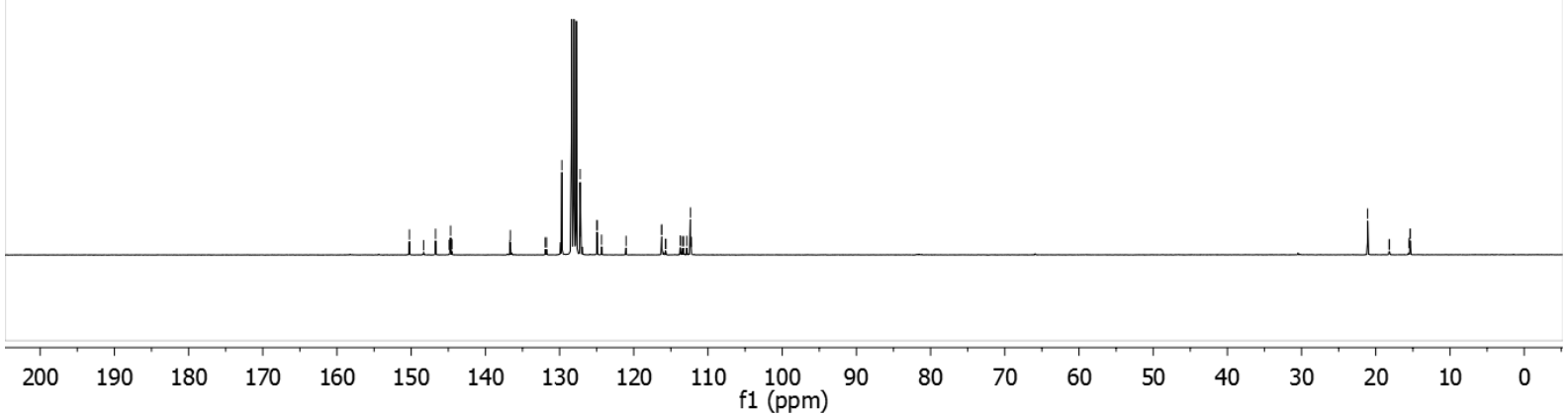


(282 MHz, $\mathrm{C}_{6} \mathrm{D}_{6}$ )
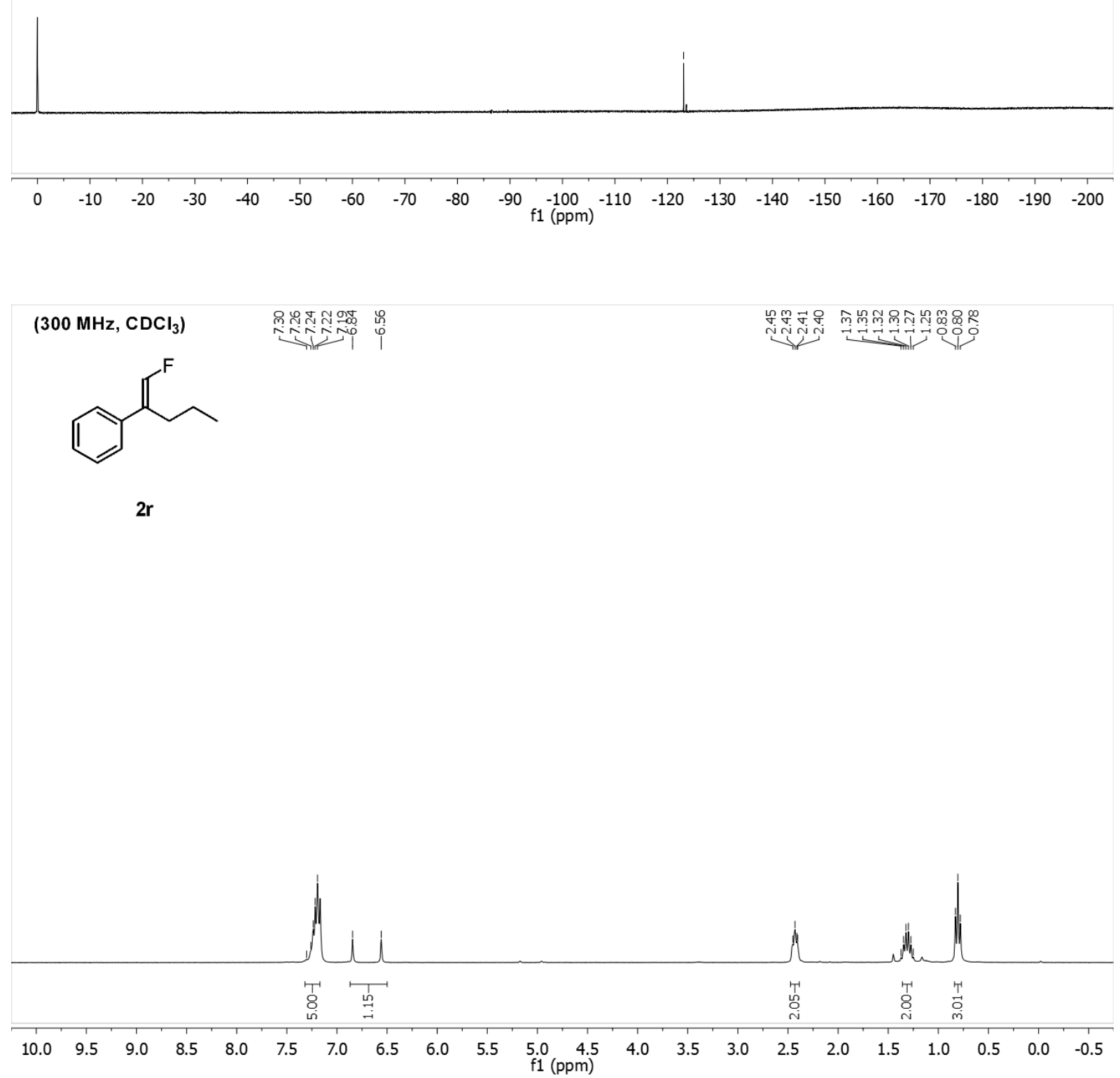

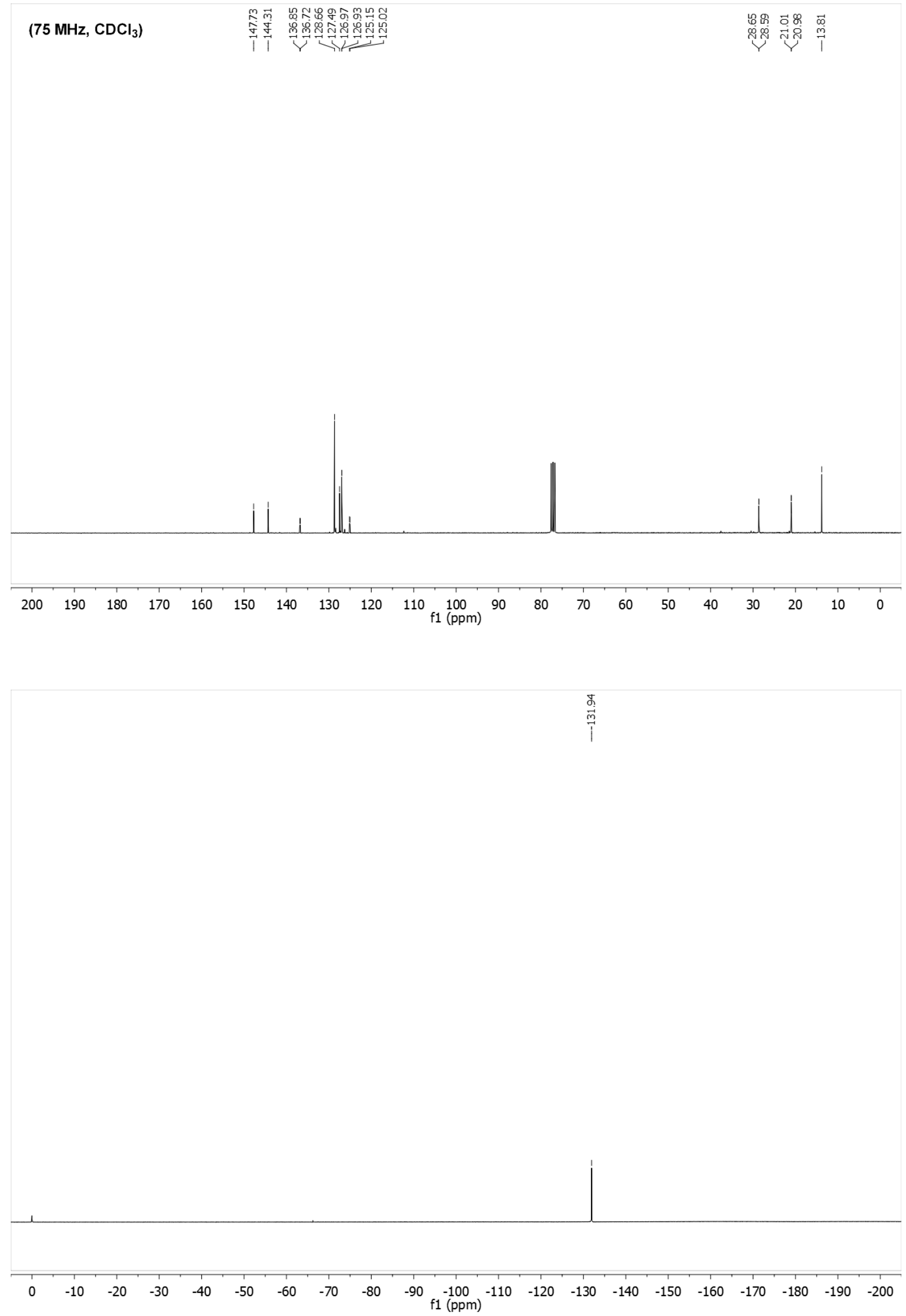


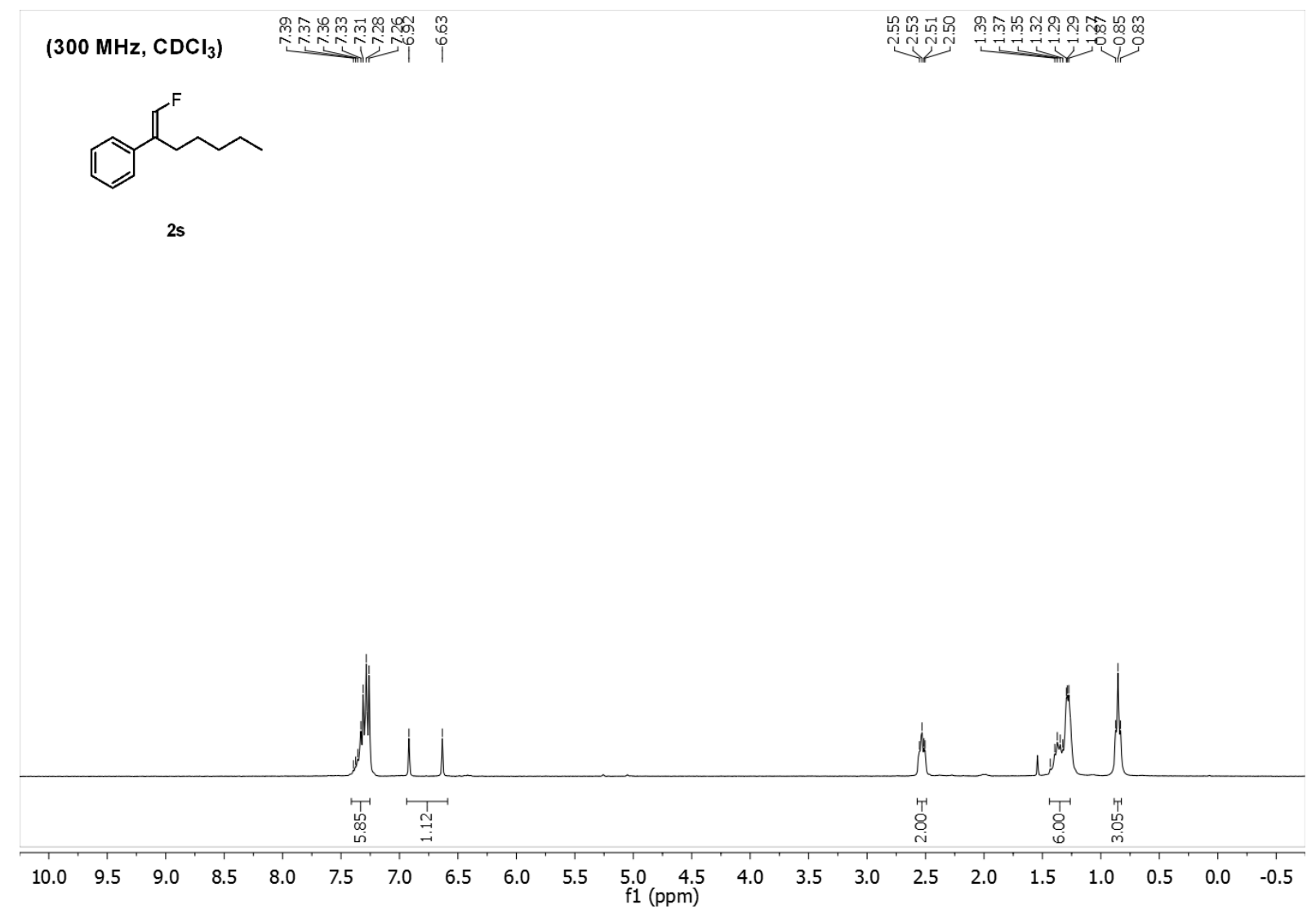

(75 MHz, $\mathrm{CDCl}_{3}$ )

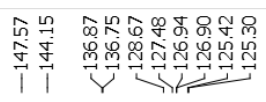

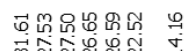

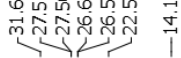

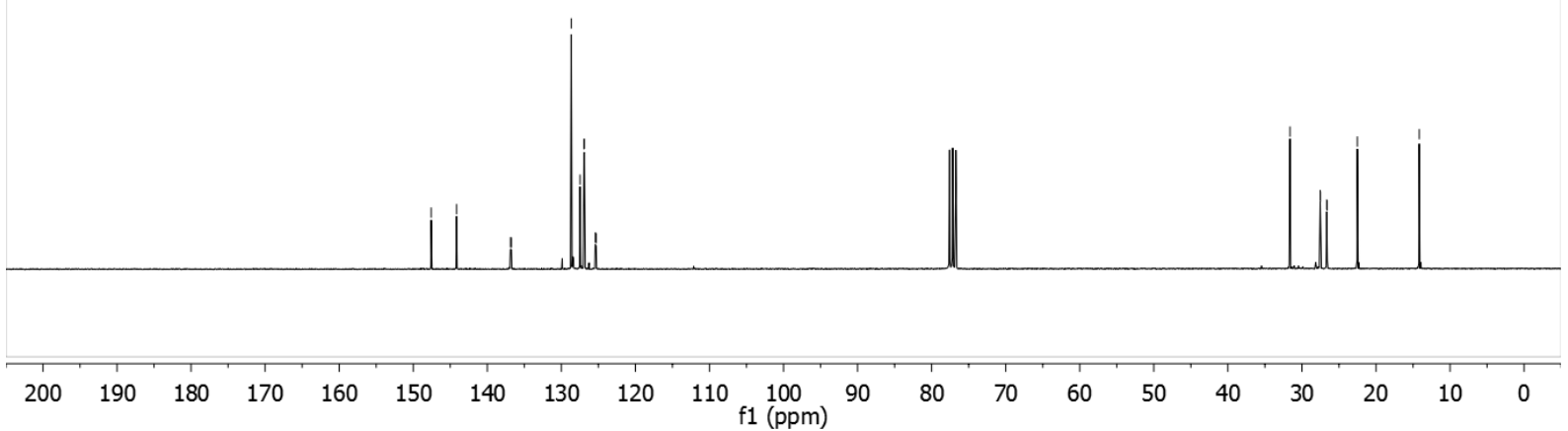




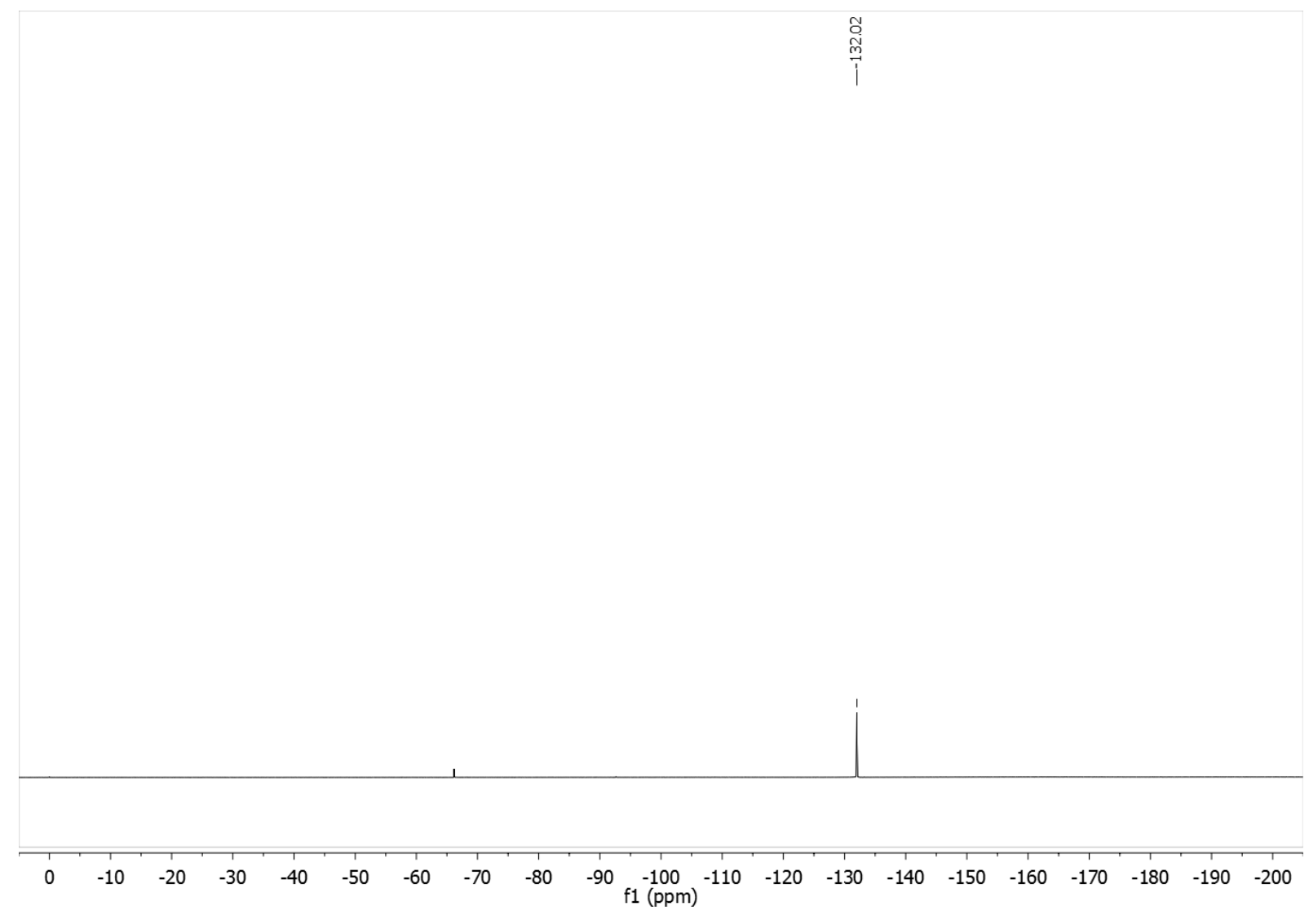

(300 MHz, $\left.\mathrm{CDCl}_{3}\right)$

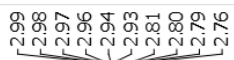<smiles>FC=C(CCc1ccccc1)c1ccccc1</smiles>

2t

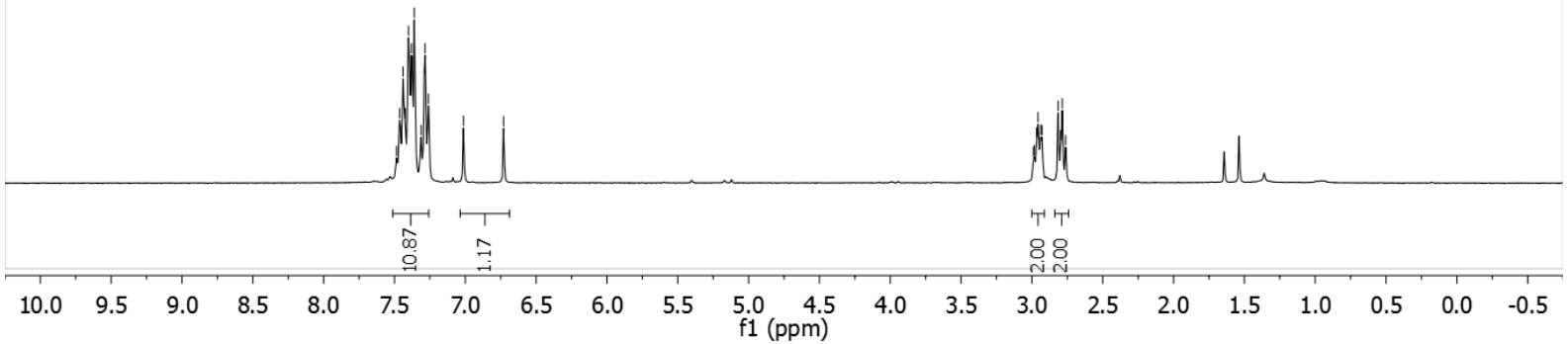



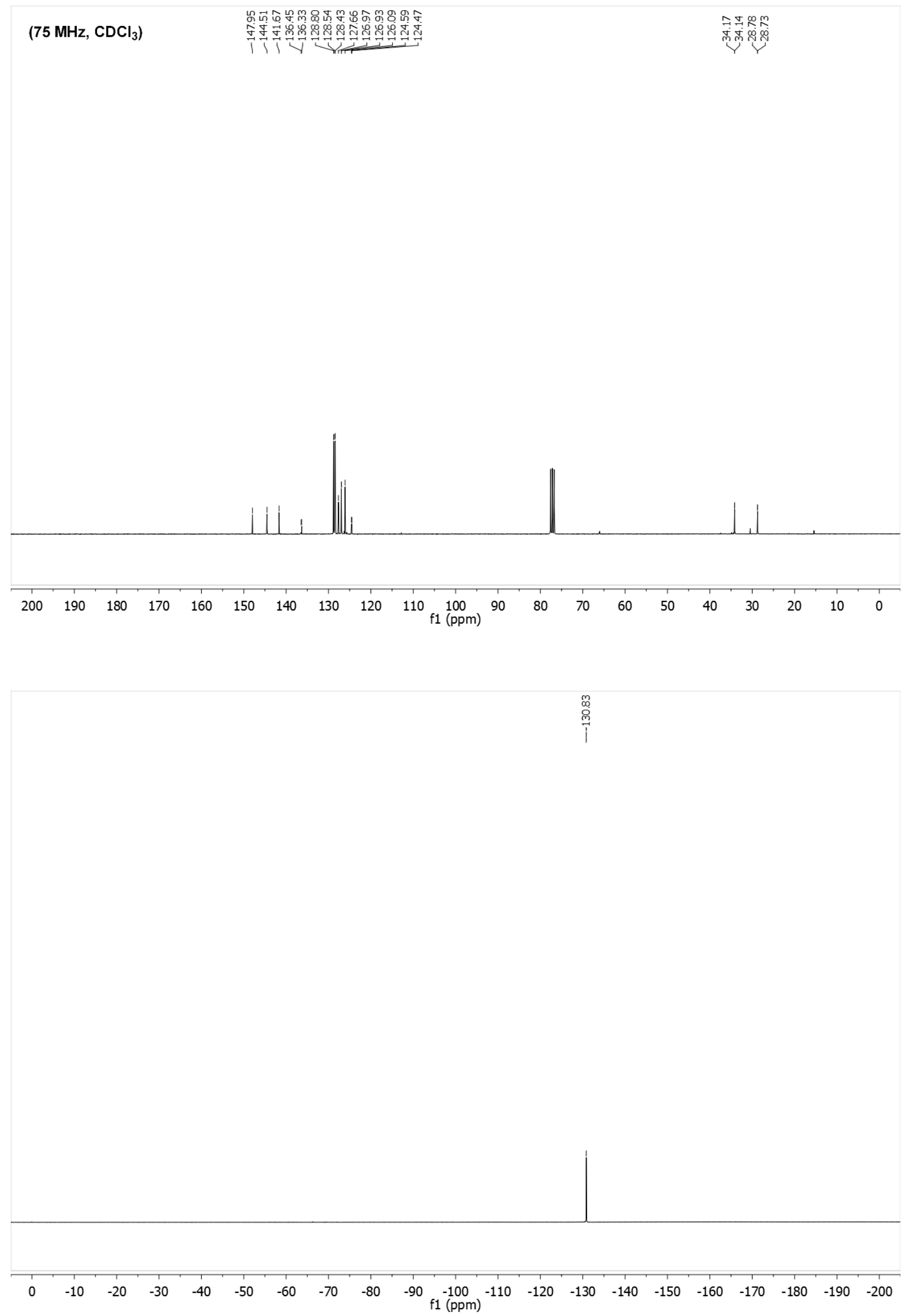


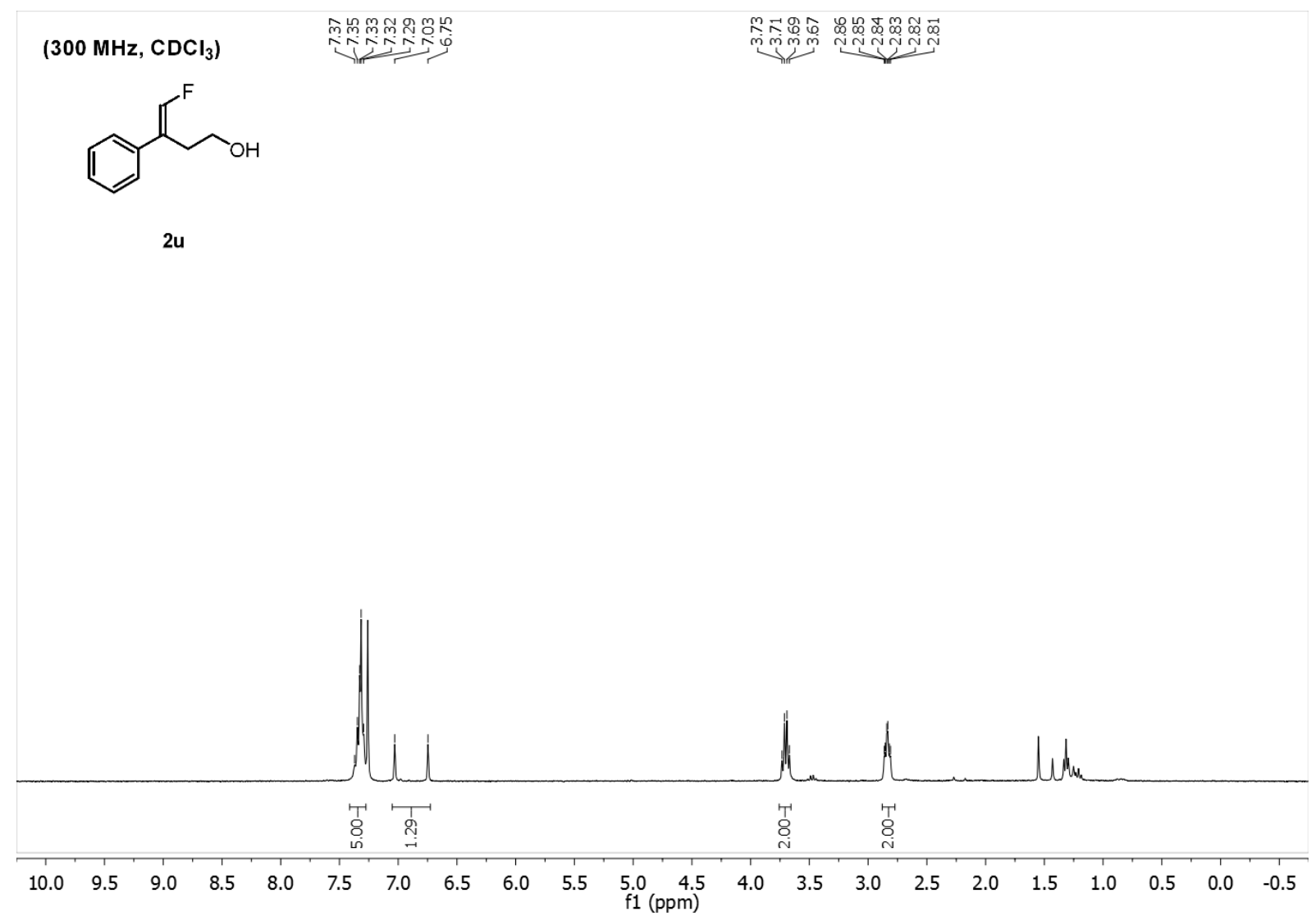

$\left(75 \mathrm{MHz}, \mathrm{CDCl}_{3}\right) \quad$ V

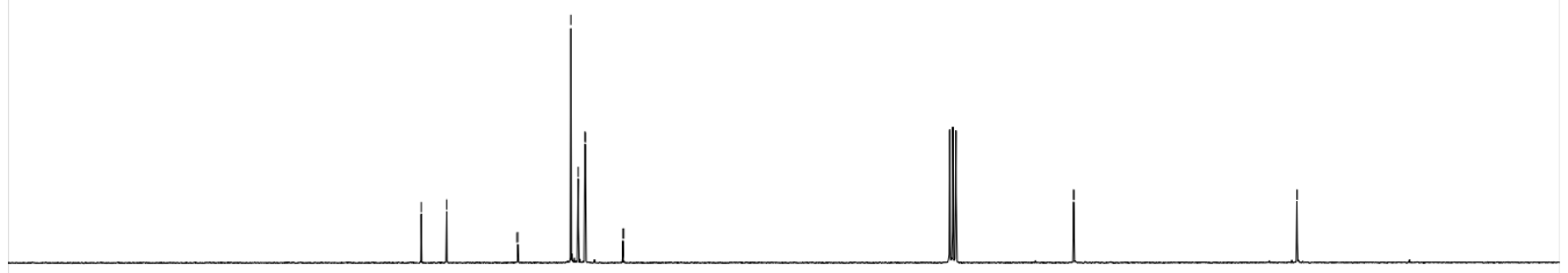

$\begin{array}{lllllllllllllllllllll}200 & 190 & 180 & 170 & 160 & 150 & 140 & 130 & 120 & 110 & 100 & 90 & 80 & 70 & 60 & 50 & 40 & 30 & 20 & 10 & 0\end{array}$ 

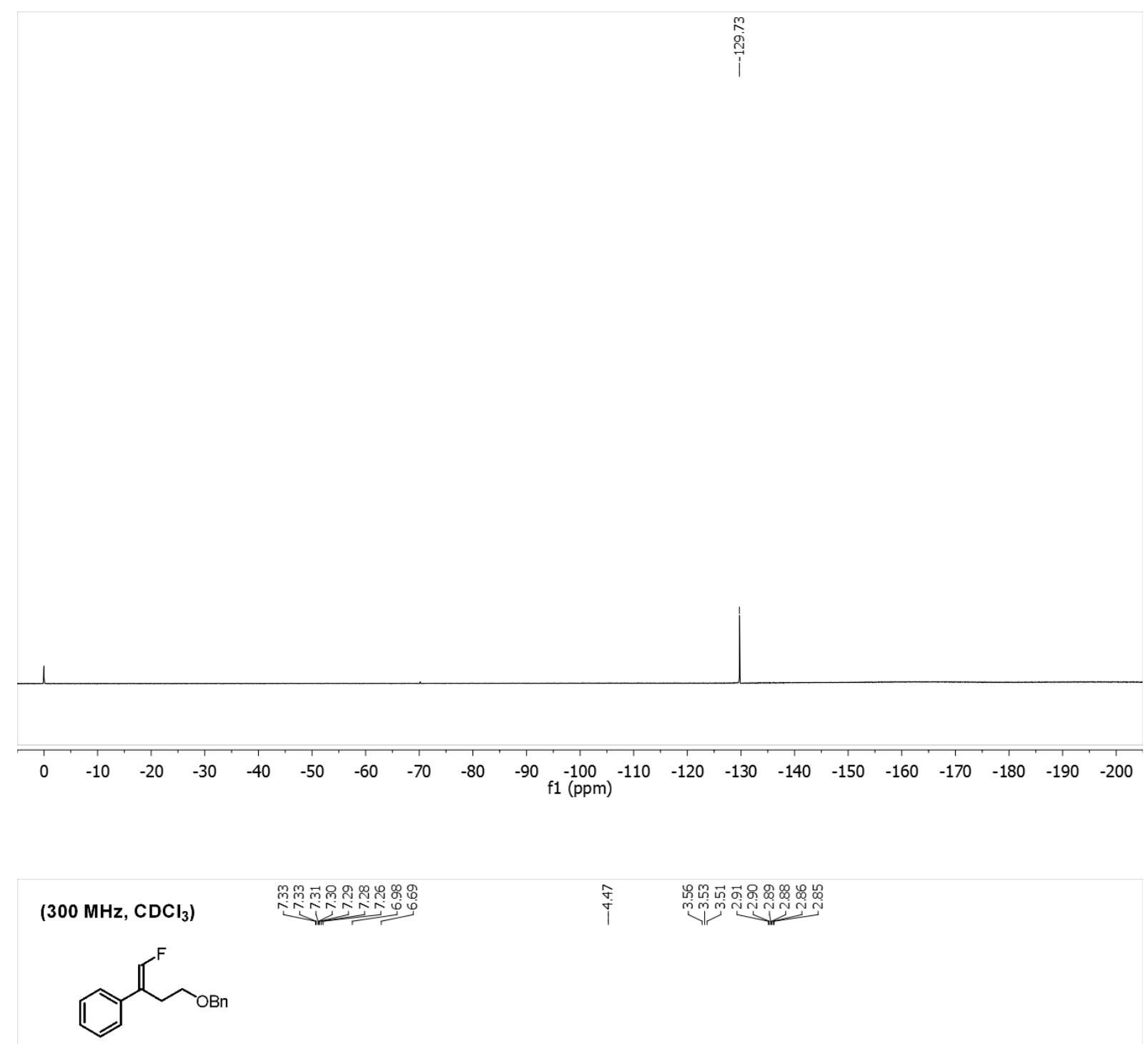

$2 v$

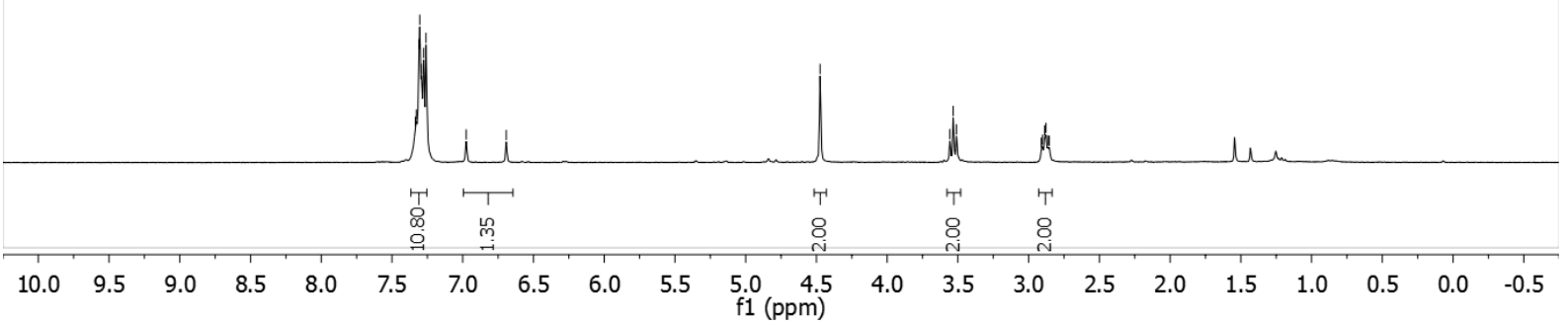



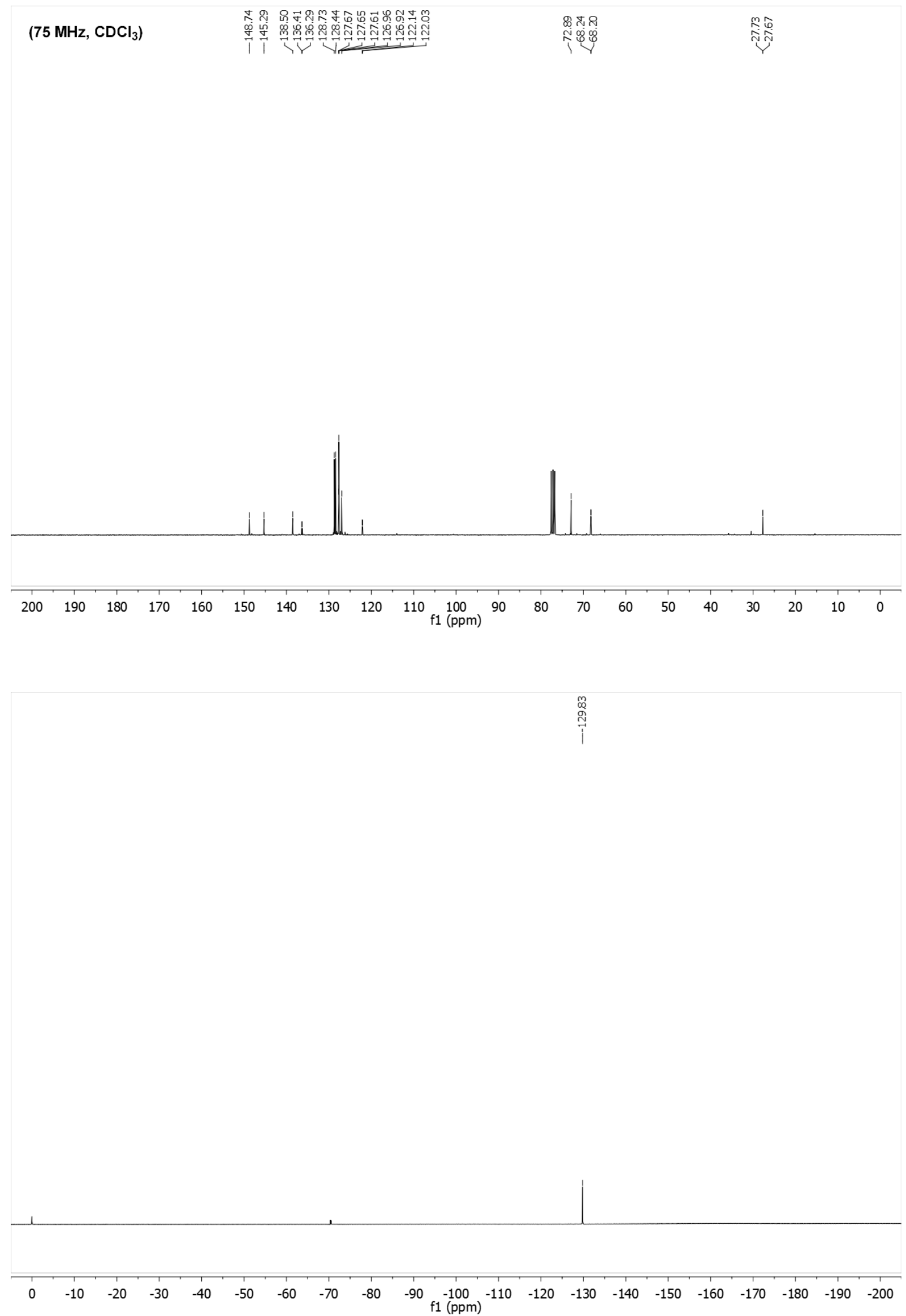


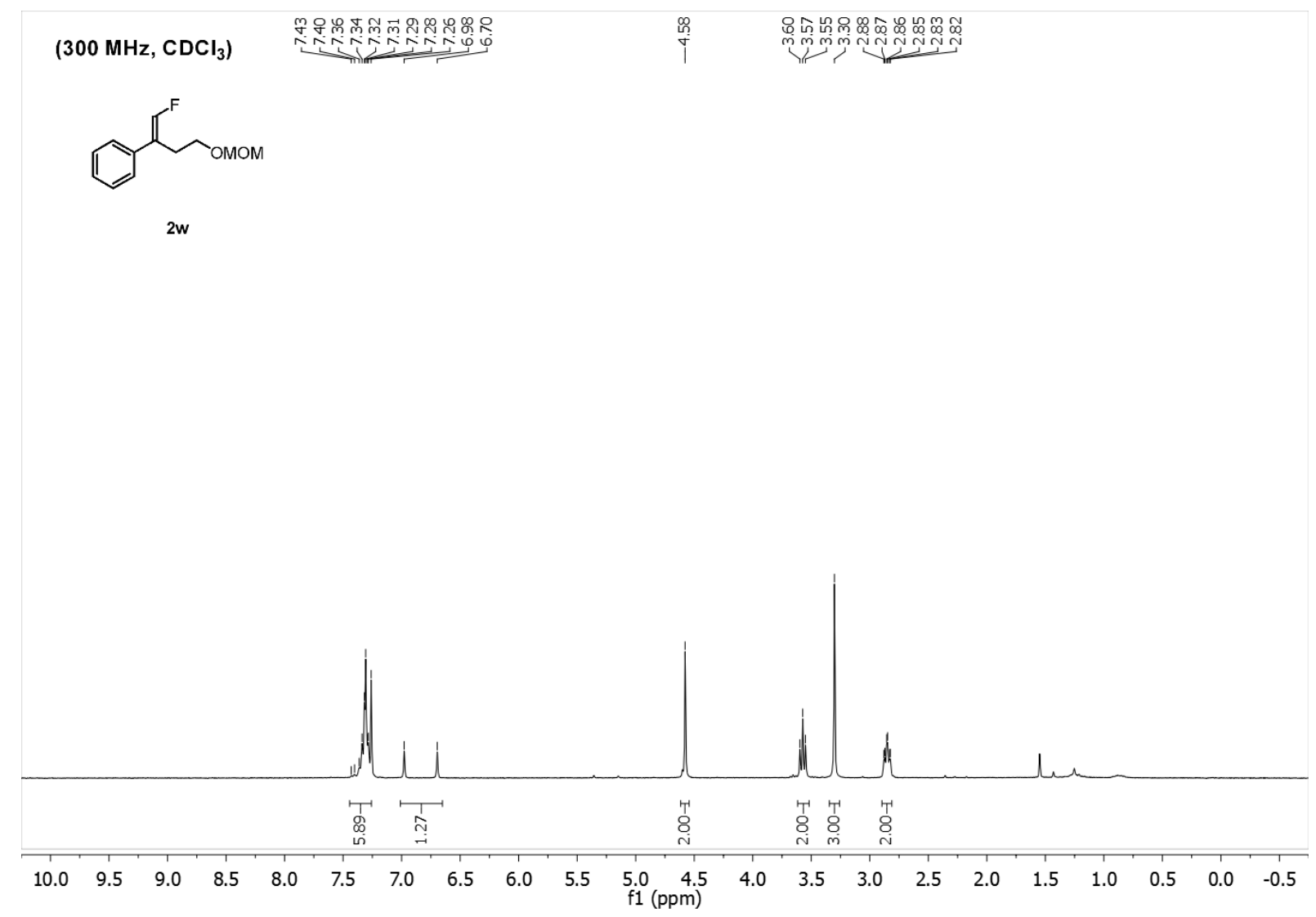

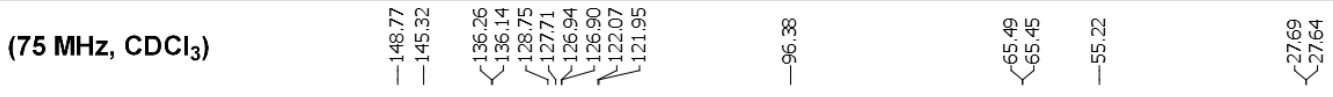

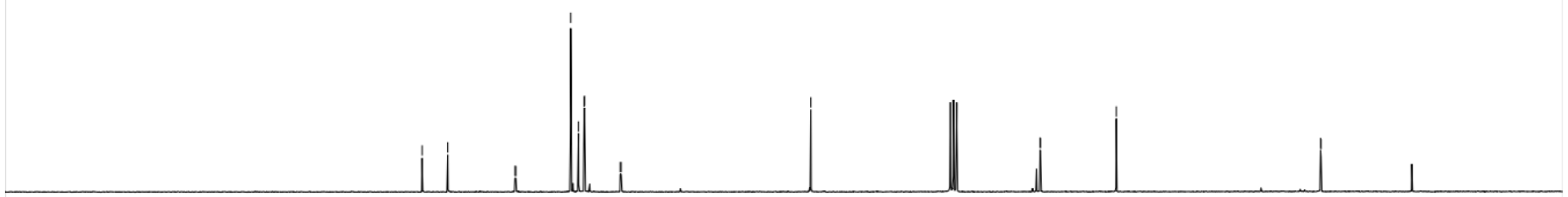

$\begin{array}{lllllllllllllllllllllll}200 & 190 & 180 & 170 & 160 & 150 & 140 & 130 & 120 & 110 & 100 & 90 & 80 & 70 & 60 & 50 & 40 & 30 & 20 & 10 & 0\end{array}$ 
(282 $\mathrm{MHz}, \mathrm{CDCl}_{3}$ )

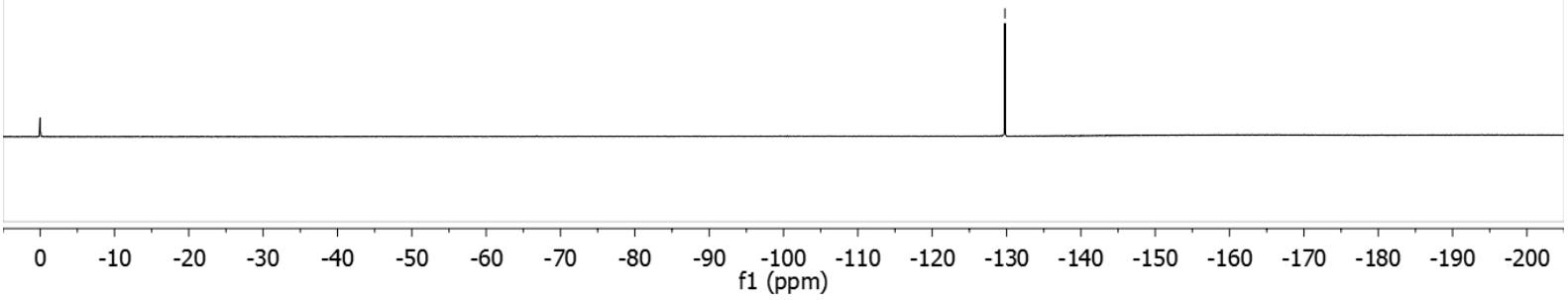

(300 MHz, $\left.\mathrm{CDCl}_{3}\right)$

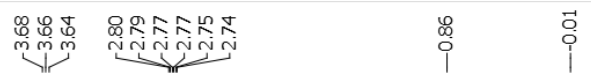<smiles>COCCC(=CF)c1ccccc1</smiles>

$2 x$

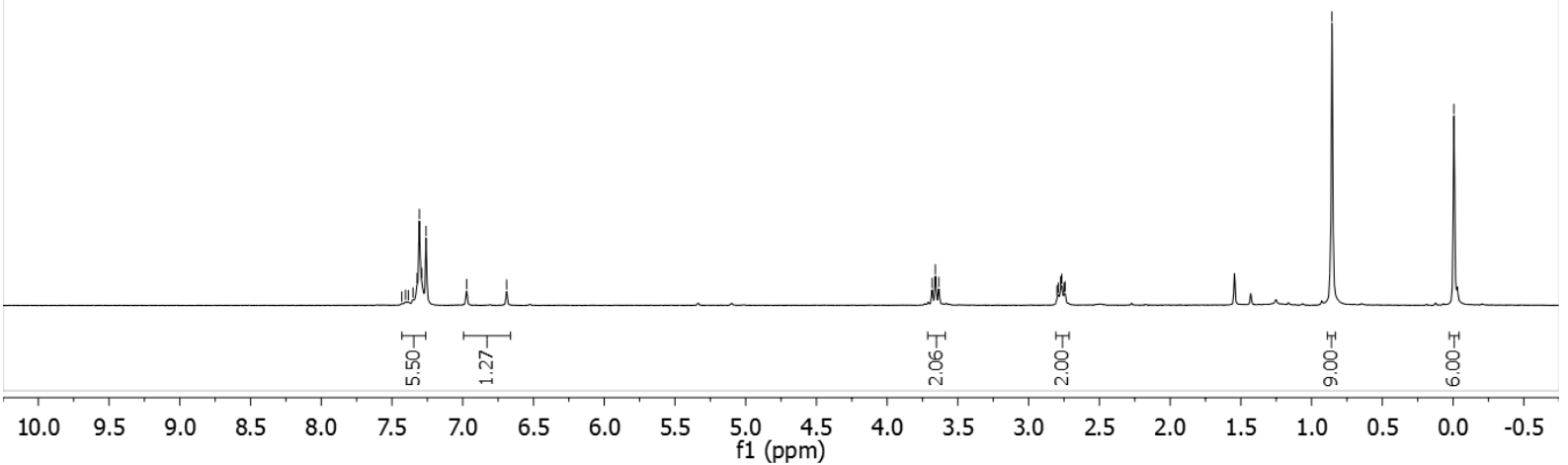




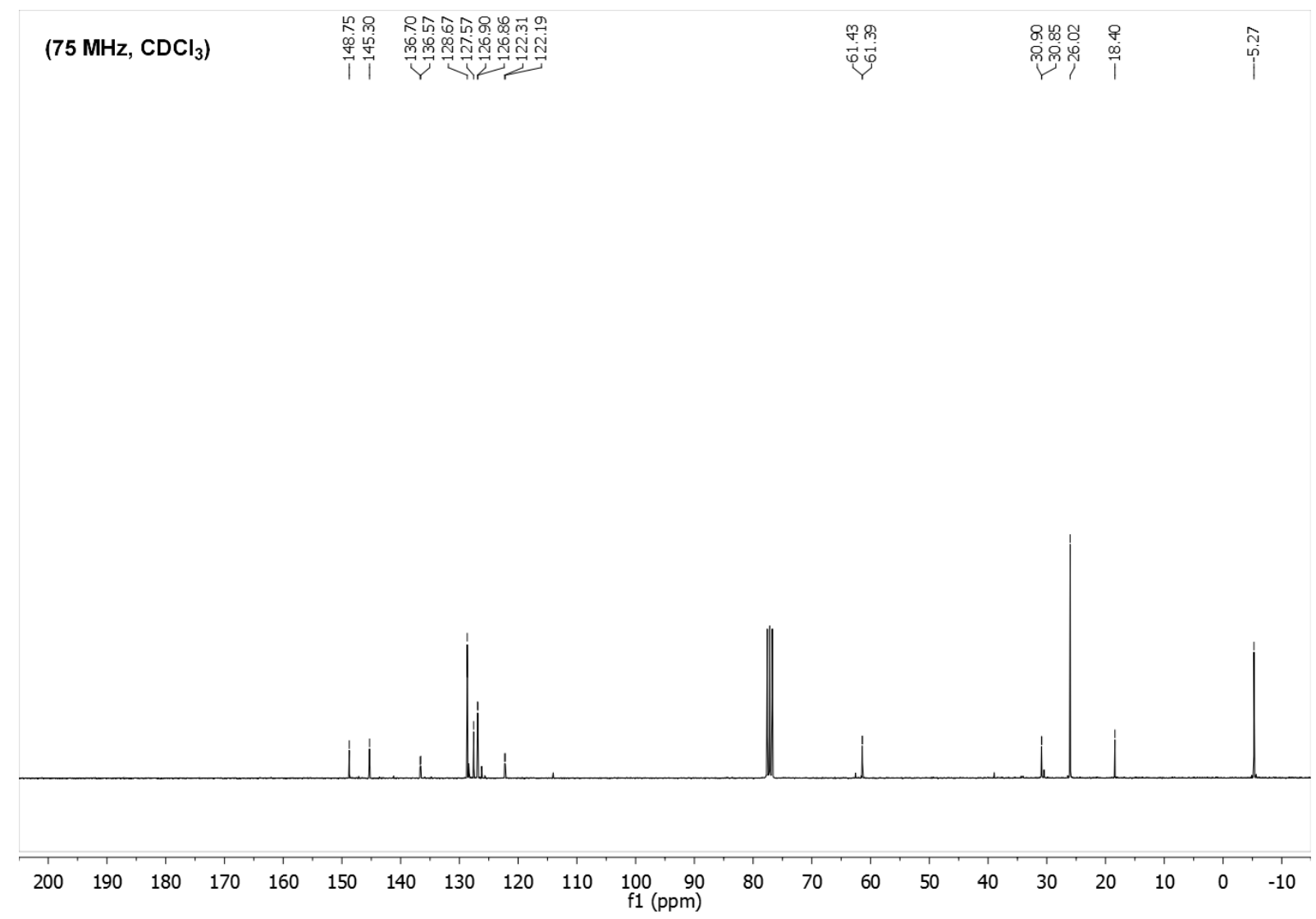

(282 MHz, $\mathrm{CDCl}_{3}$ )

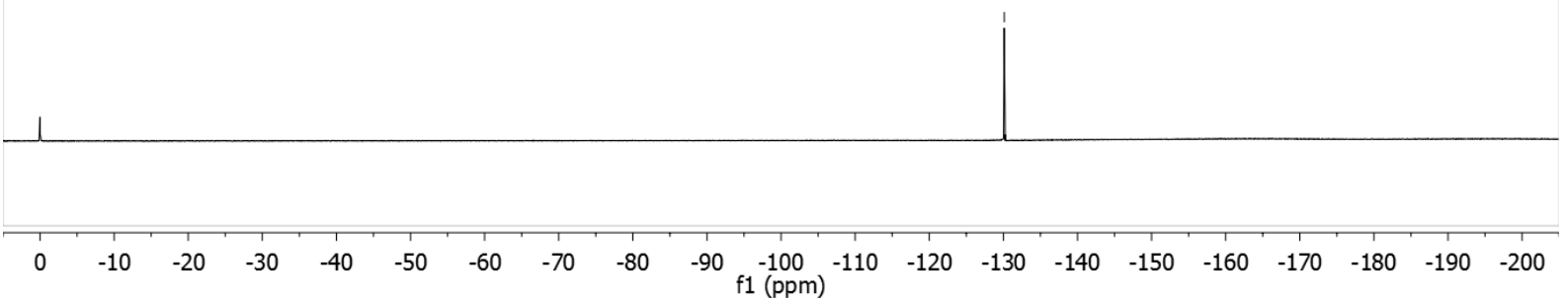




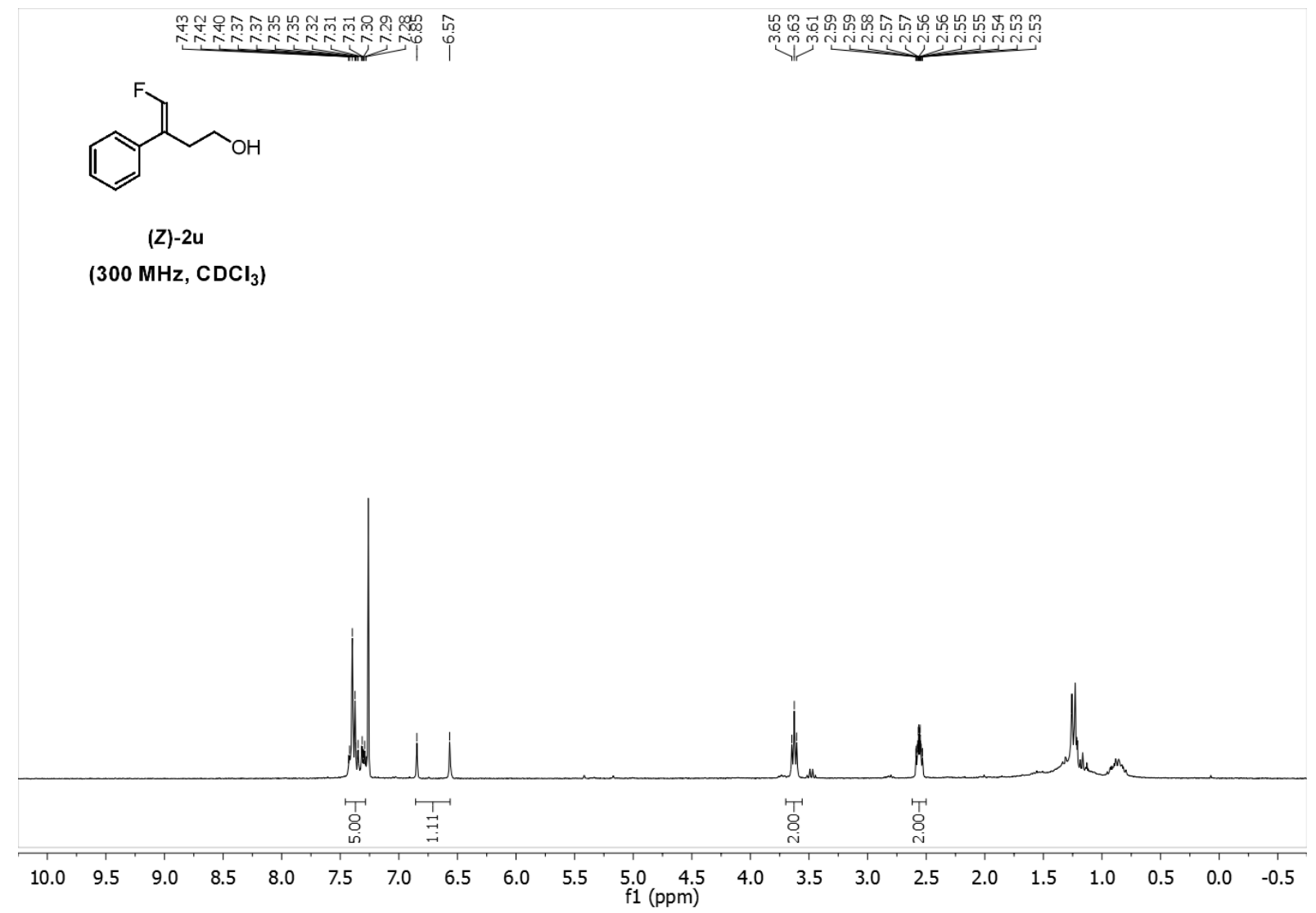

$\left(75 \mathrm{MHz}, \mathrm{CDCl}_{3}\right) \quad$ V

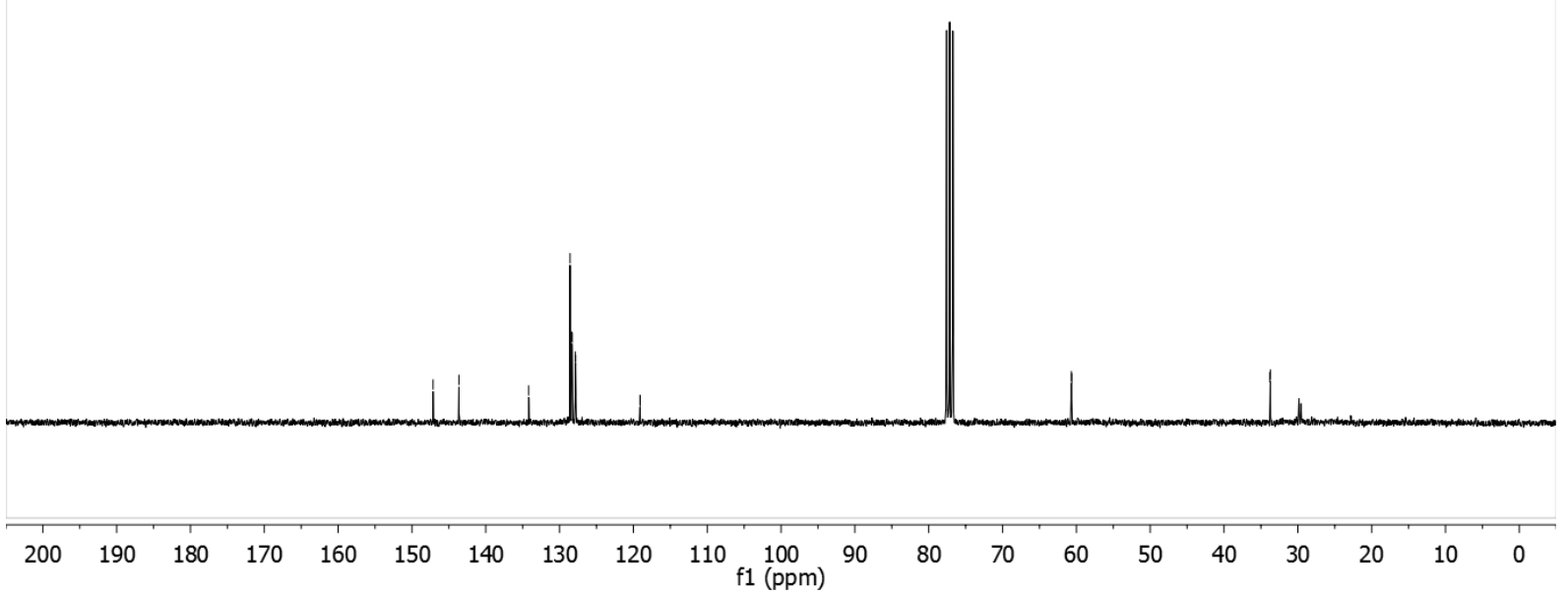


$\left(282 \mathrm{MHz}, \mathrm{CDCl}_{3}\right)$

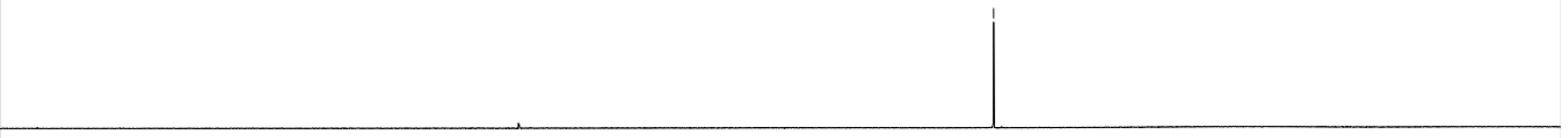

$\left(300 \mathrm{MHz}, \mathrm{CDCl}_{3}\right)$

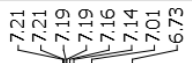

$\underbrace{N O m^{\infty}}$

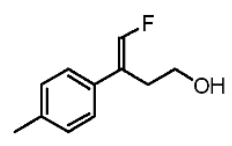

$(E)-2 y$

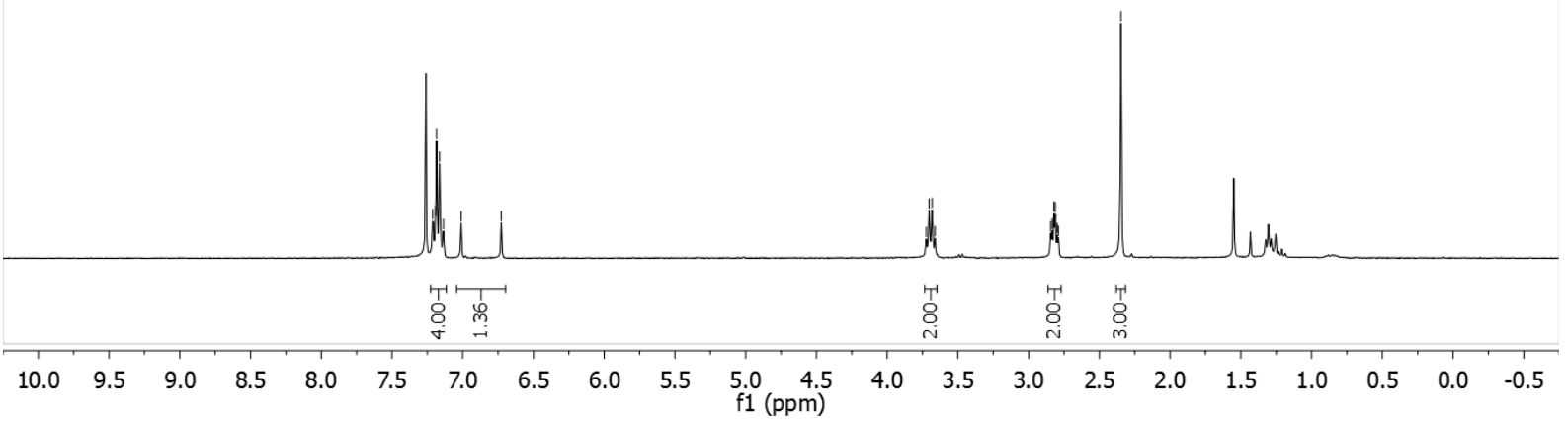




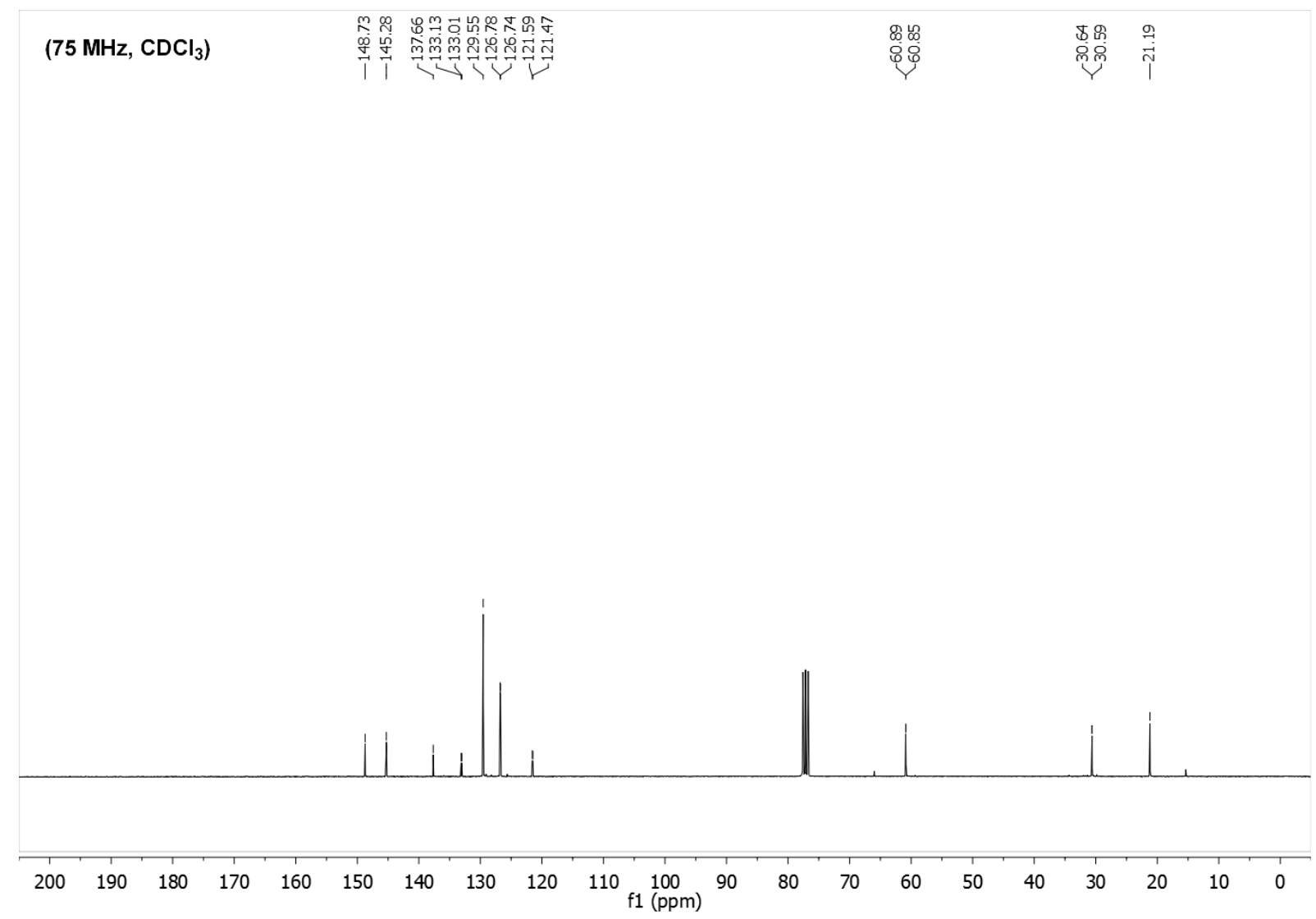

$\left(282 \mathrm{MHz}, \mathrm{CDCl}_{3}\right)$ 


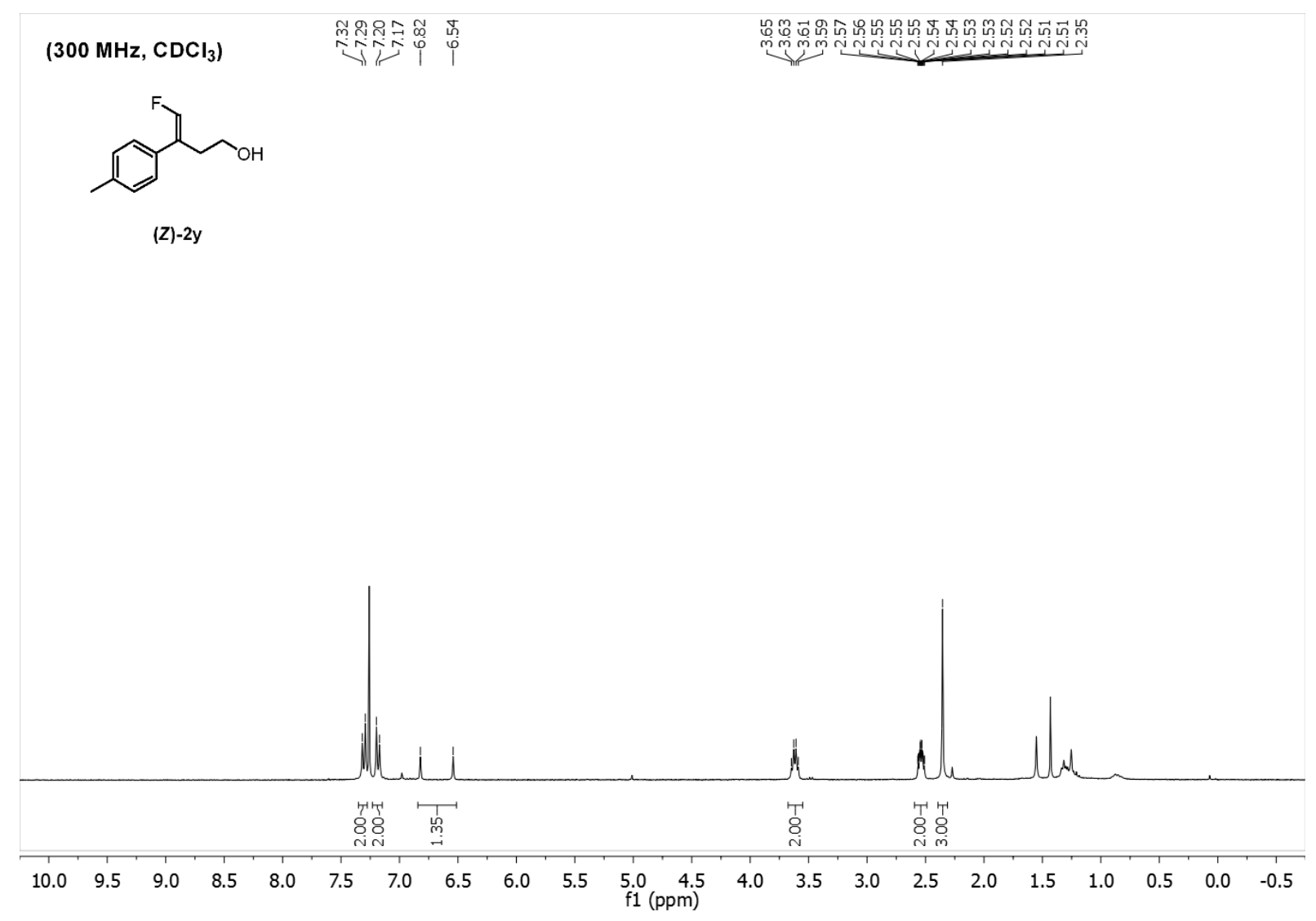

(75 MHz, $\mathrm{CDCl}_{3}$ )

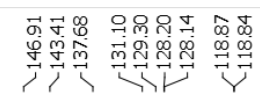

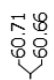

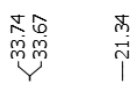

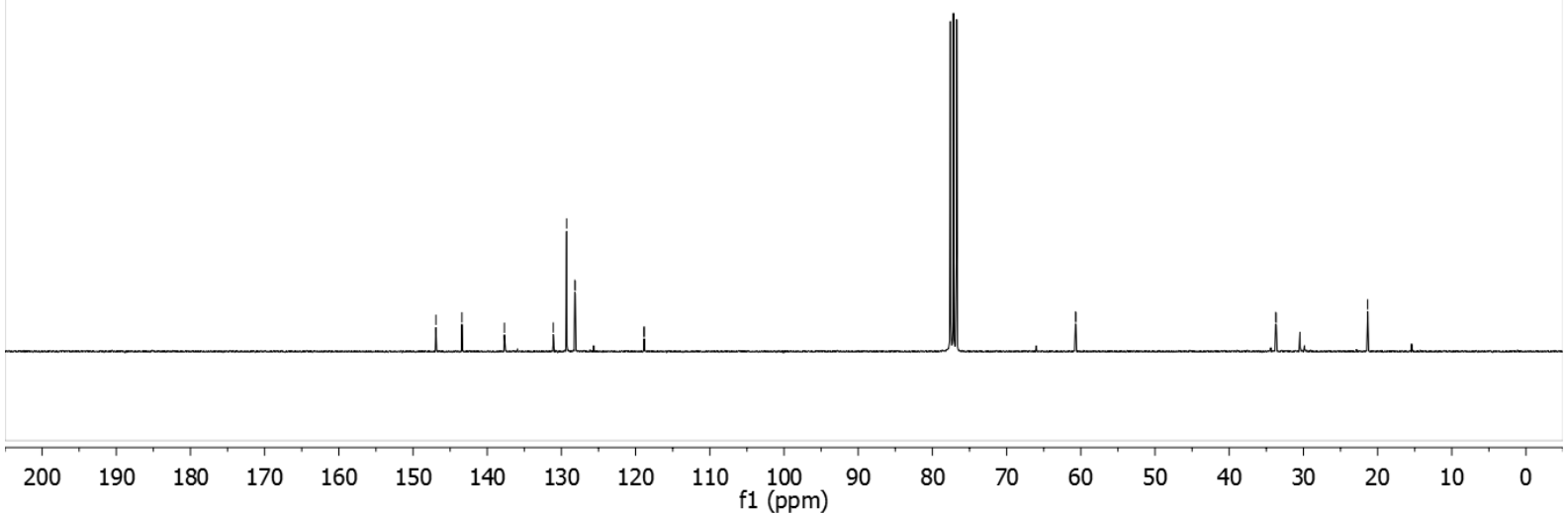


(282 MHz, $\mathrm{CDCl}_{3}$ )

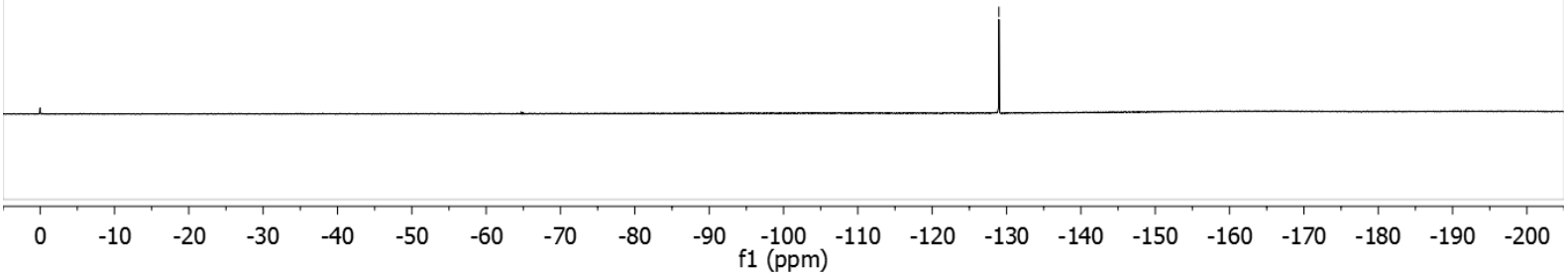

(300 MHz, $\mathrm{CDCl}_{3}$ )

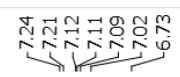

$\underbrace{m}$

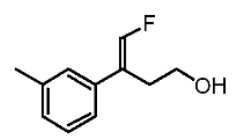

(E)-2z

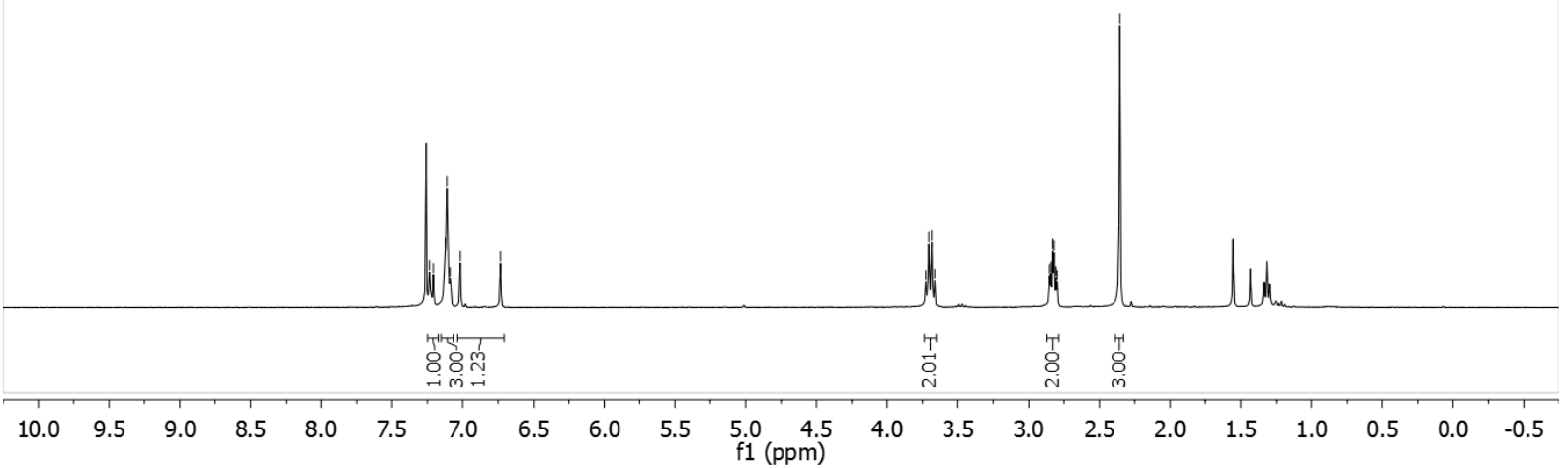




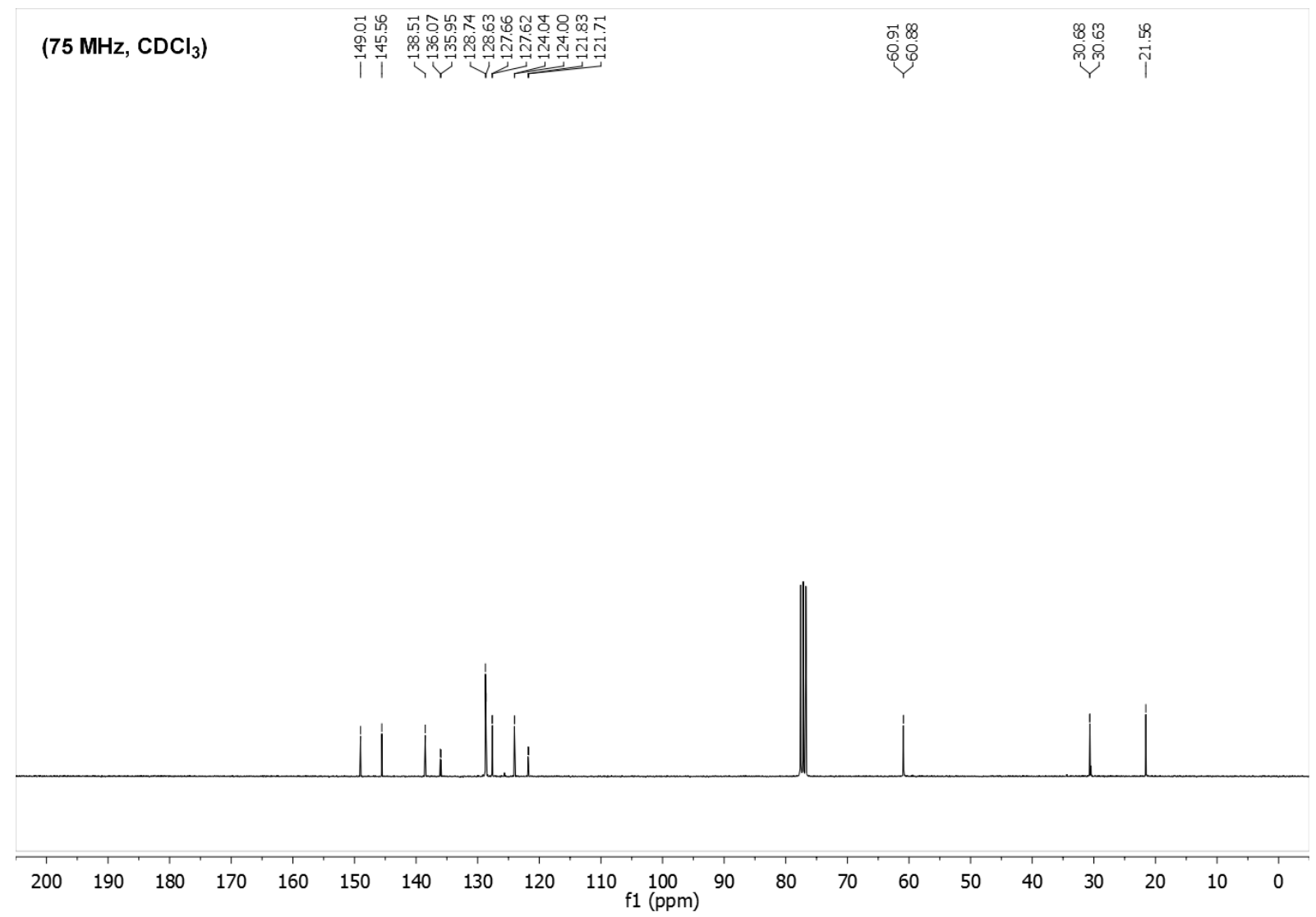

(282 MHz, $\mathrm{CDCl}_{3}$ ) 


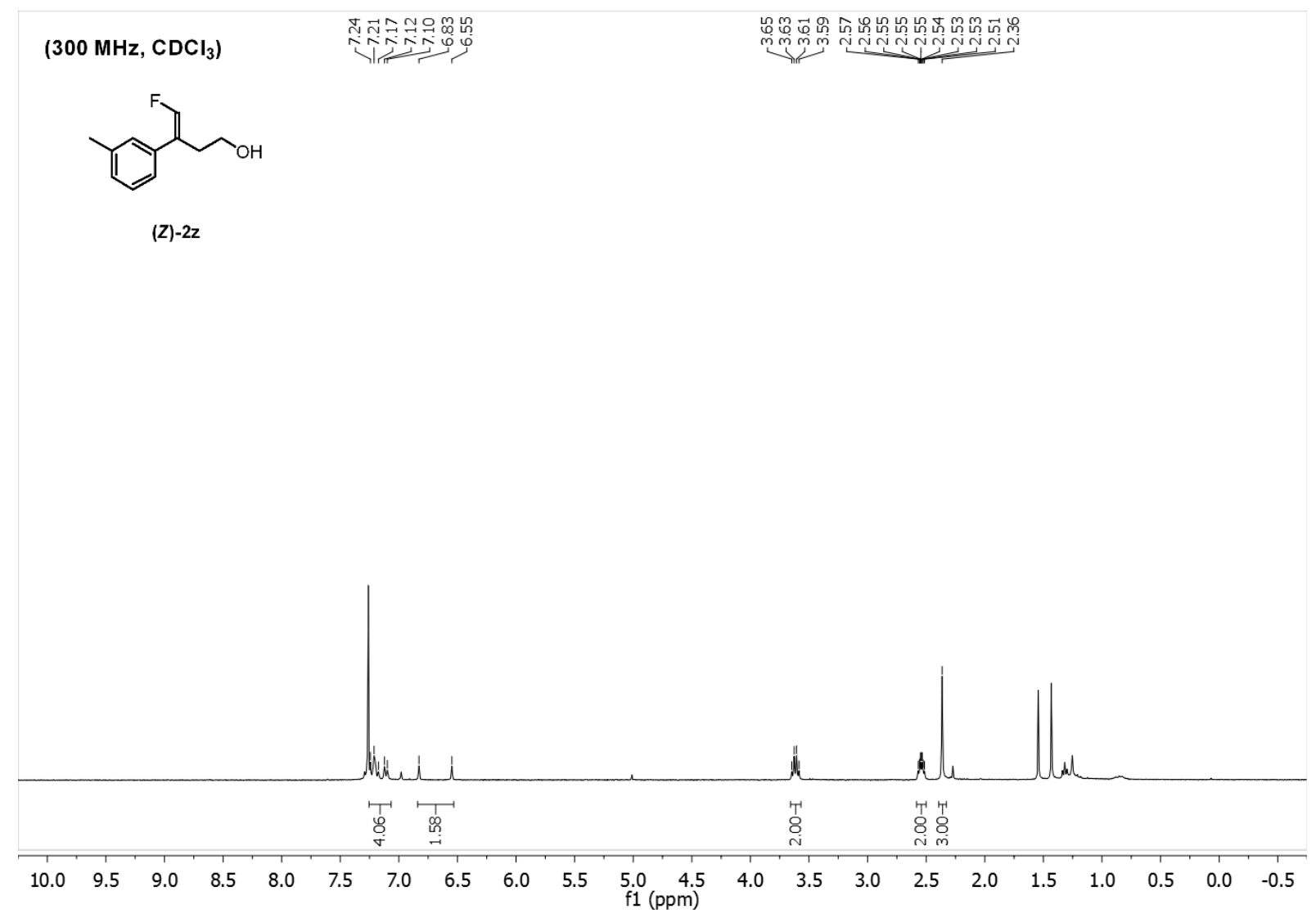

(75 MHz, $\mathrm{CDCl}_{3}$ )

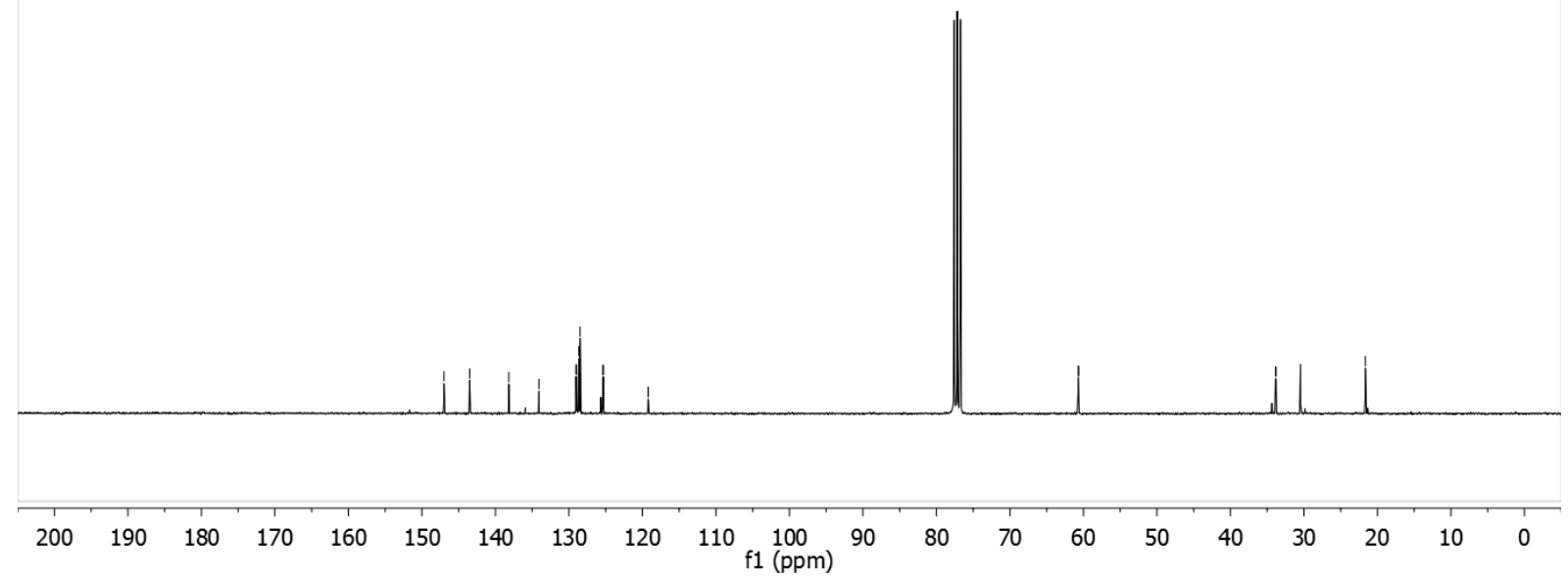


(282 MHz, $\mathrm{CDCl}_{3}$ )
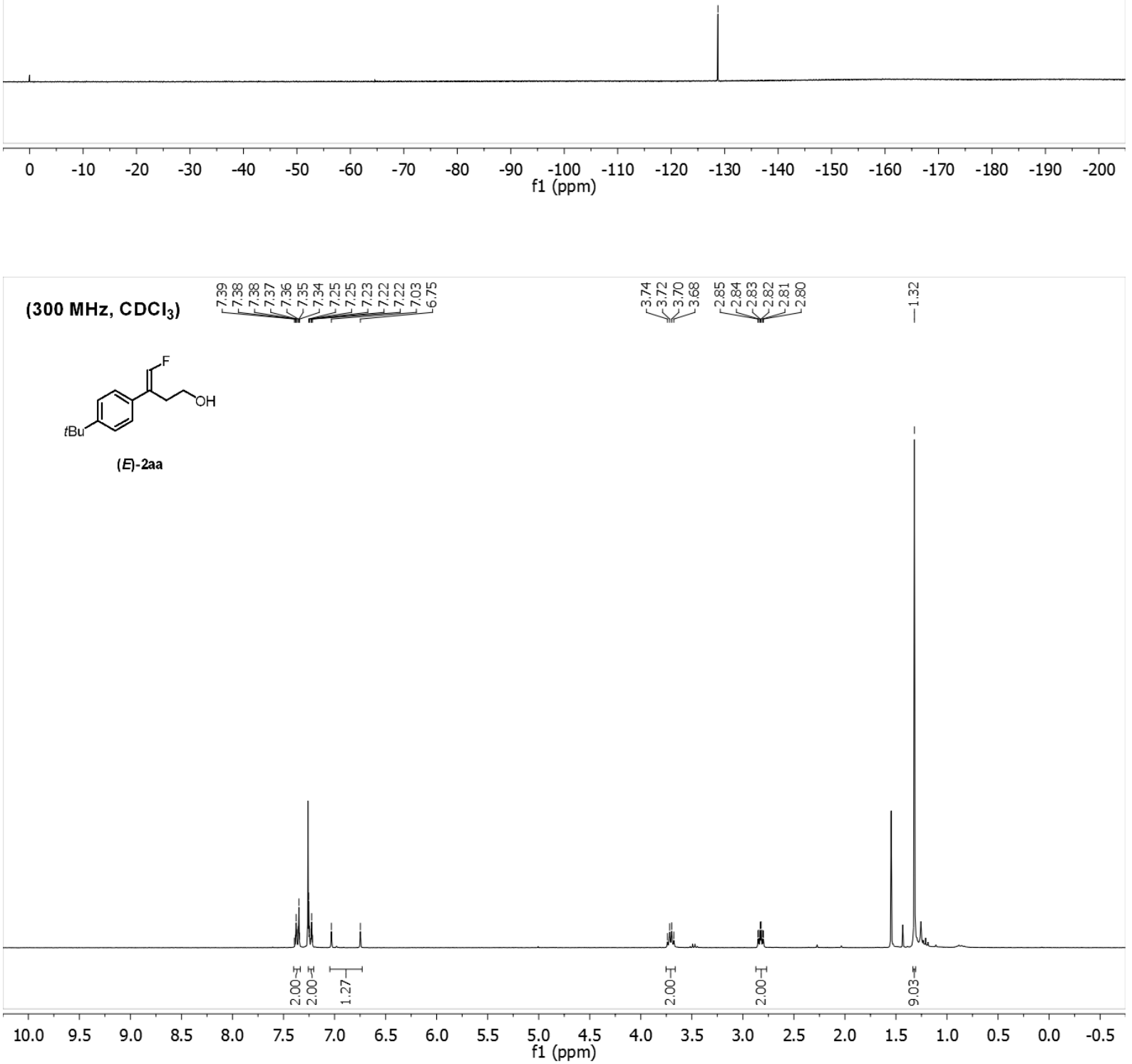


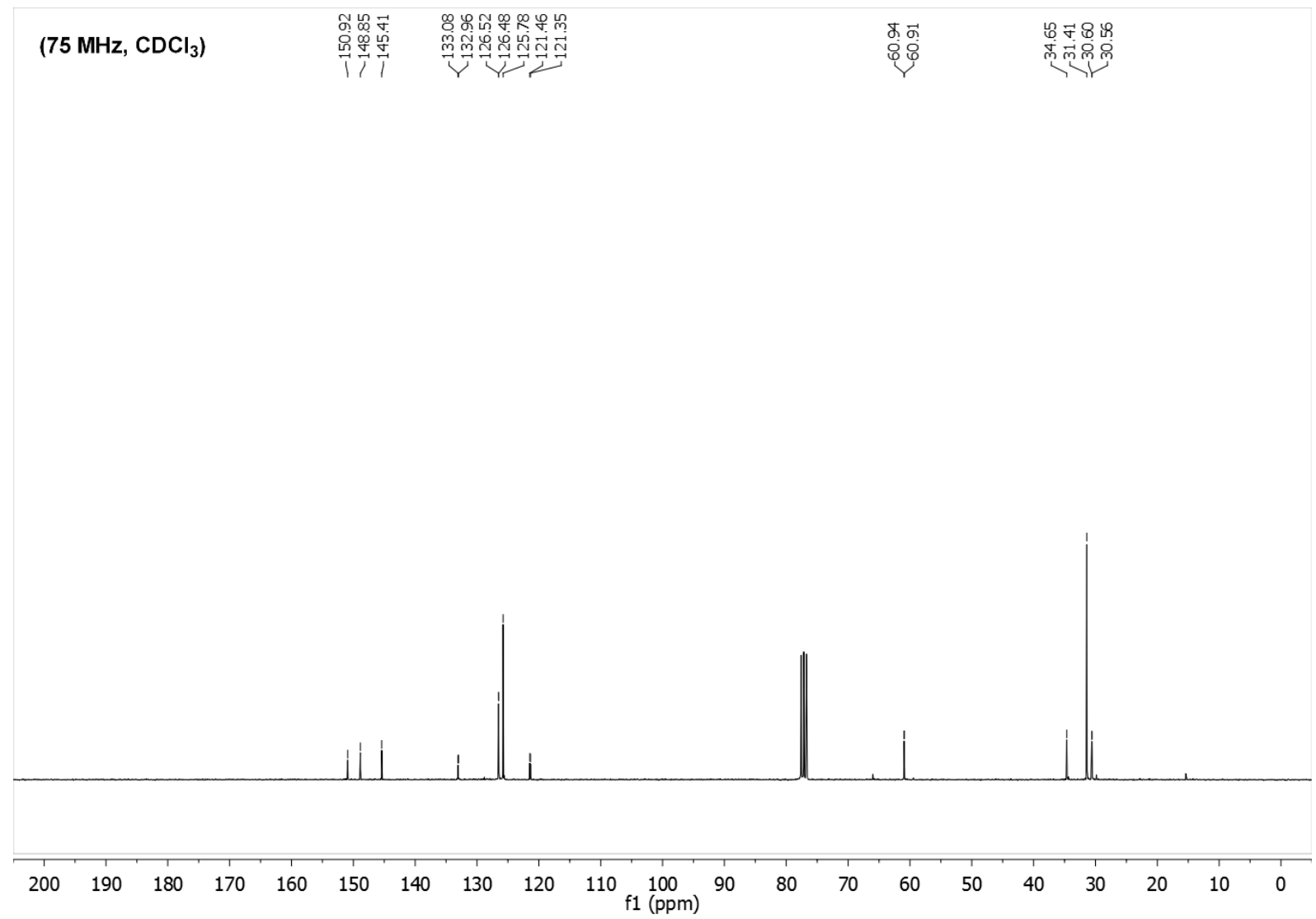

(282 MHz, $\mathrm{CDCl}_{3}$ )

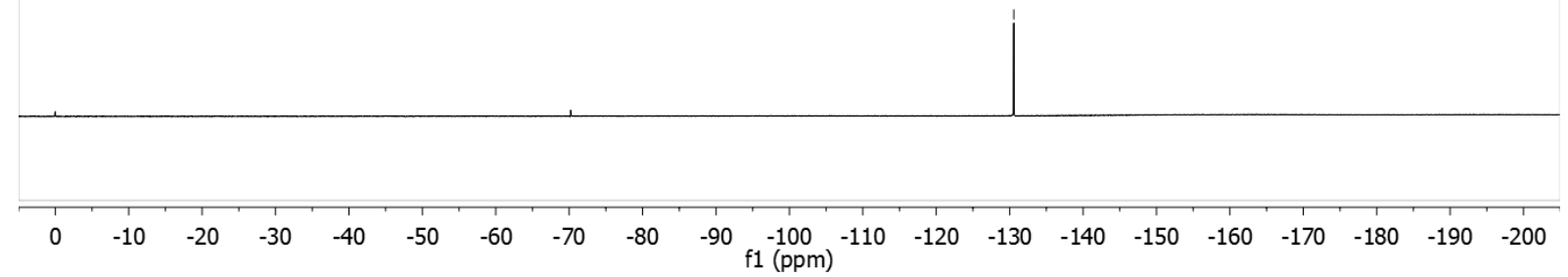




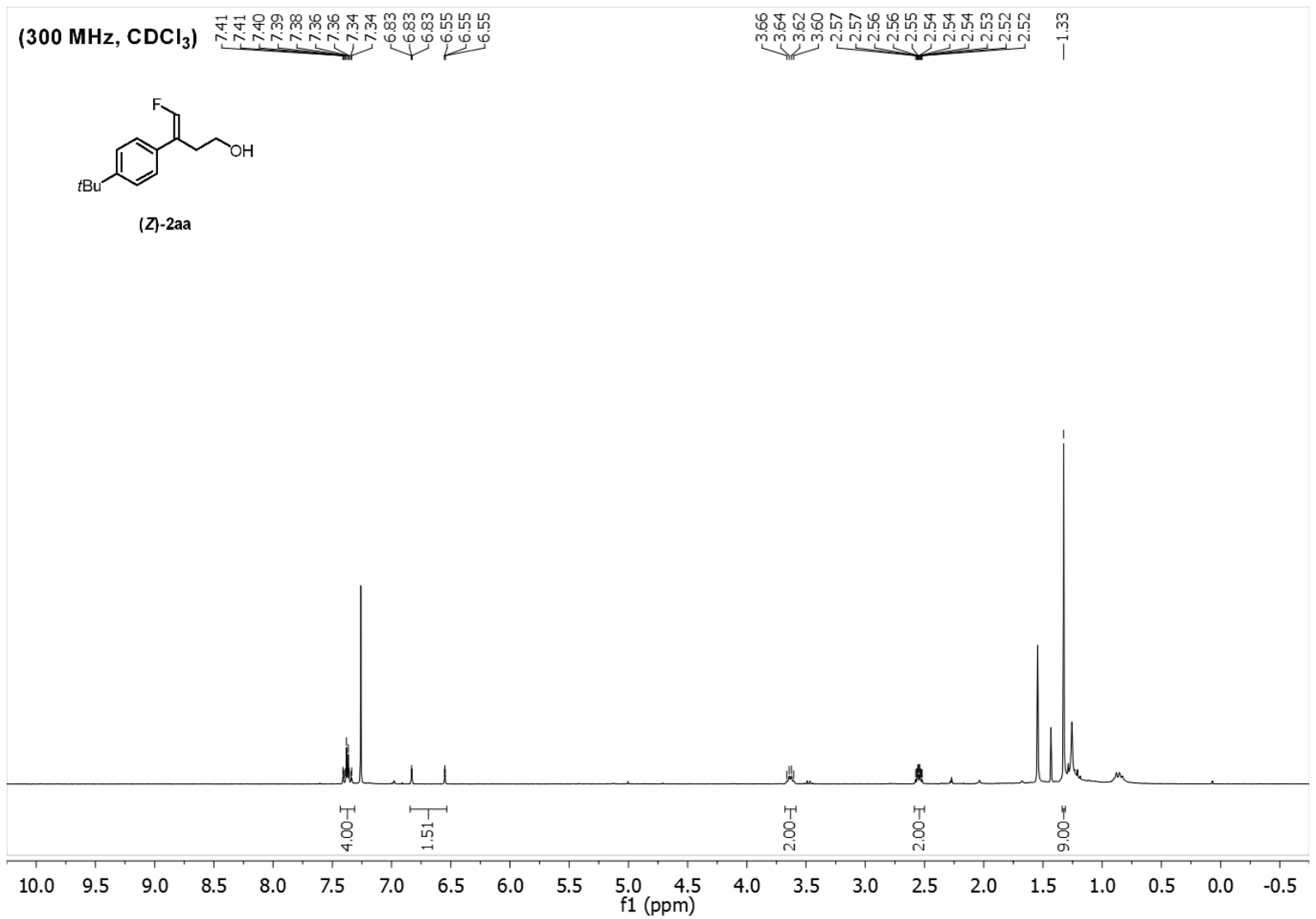

$\left(75 \mathrm{MHz}^{\mathrm{CDCl}}\right)_{3}$ )
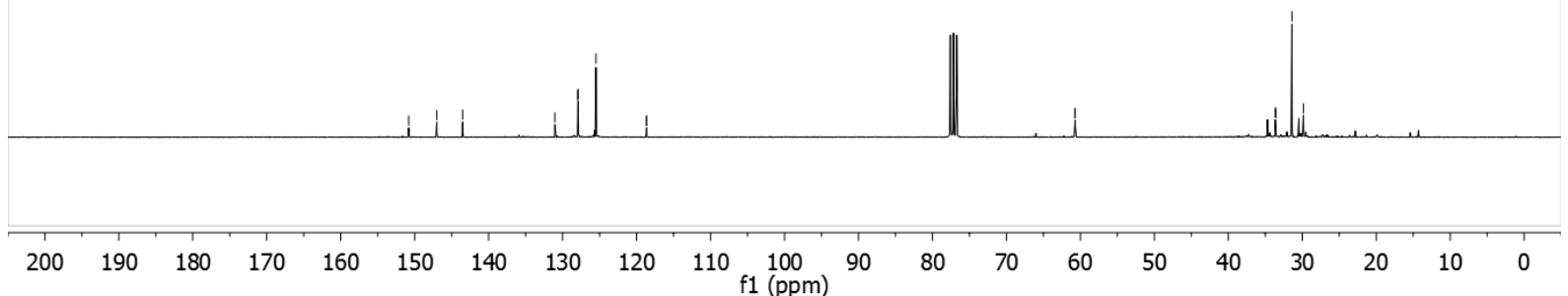
(282 MHz, $\mathrm{CDCl}_{3}$ )

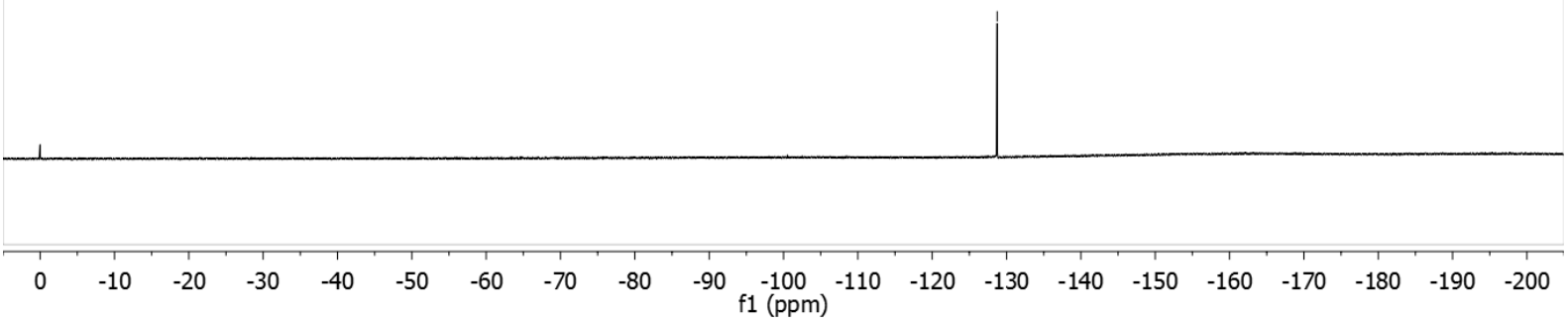

(300 MHz, $\mathrm{CDCl}_{3}$ )

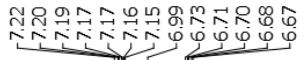<smiles>CN(C)c1ccc(/C(=C/F)CCO)cc1</smiles>

(E)-2ab

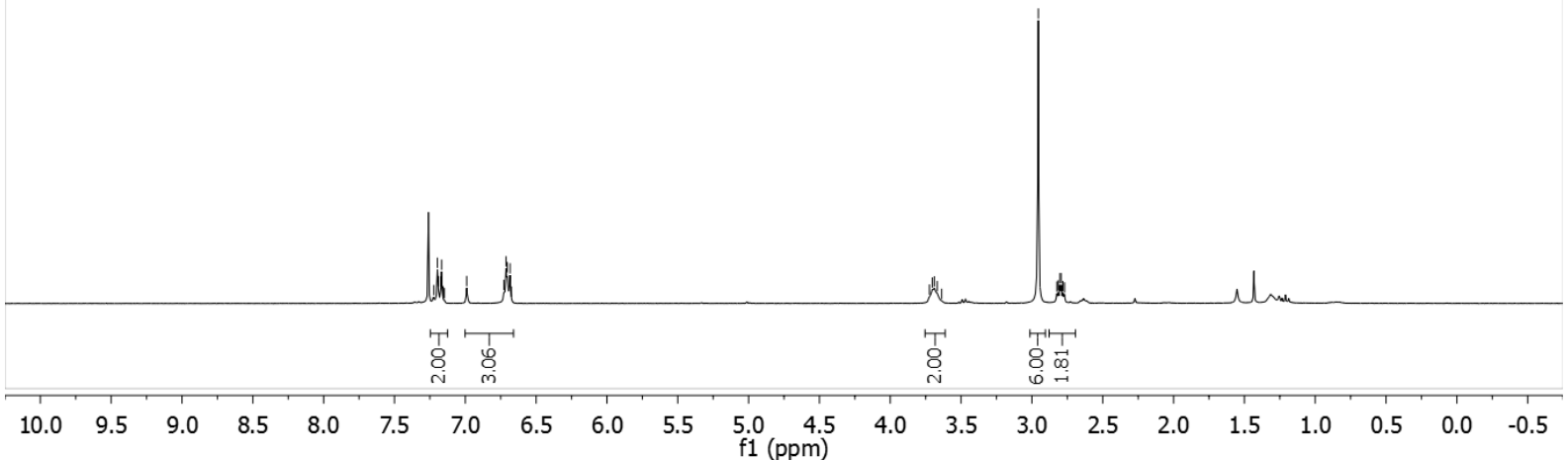




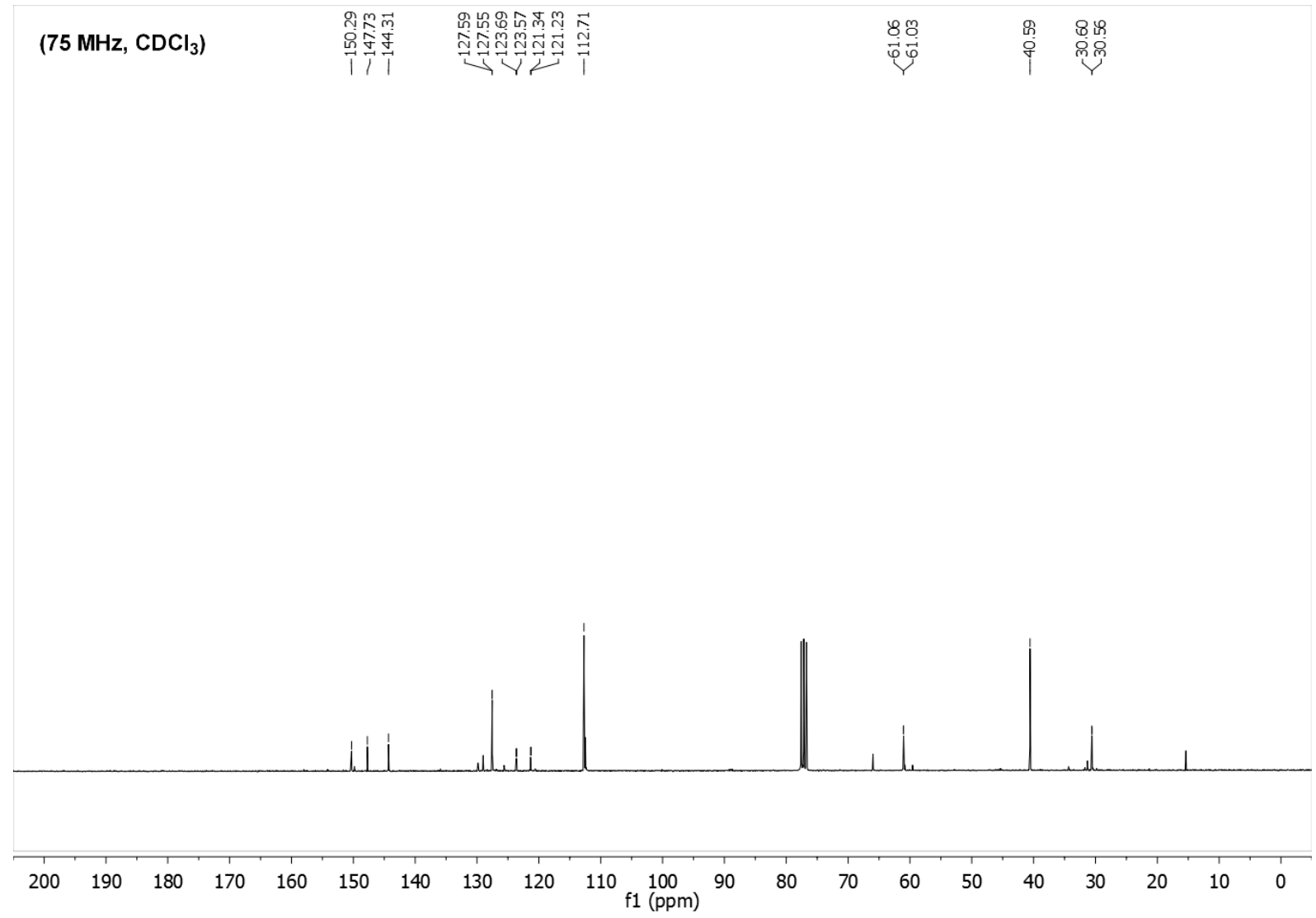

(282 MHz, $\mathrm{CDCl}_{3}$ )

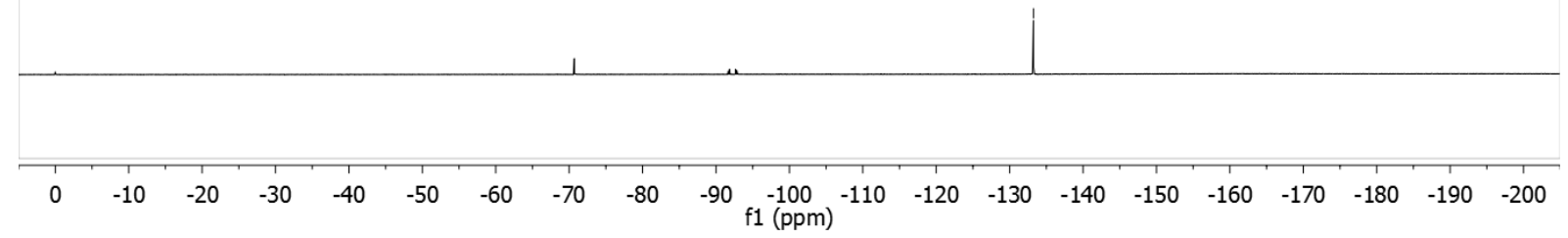




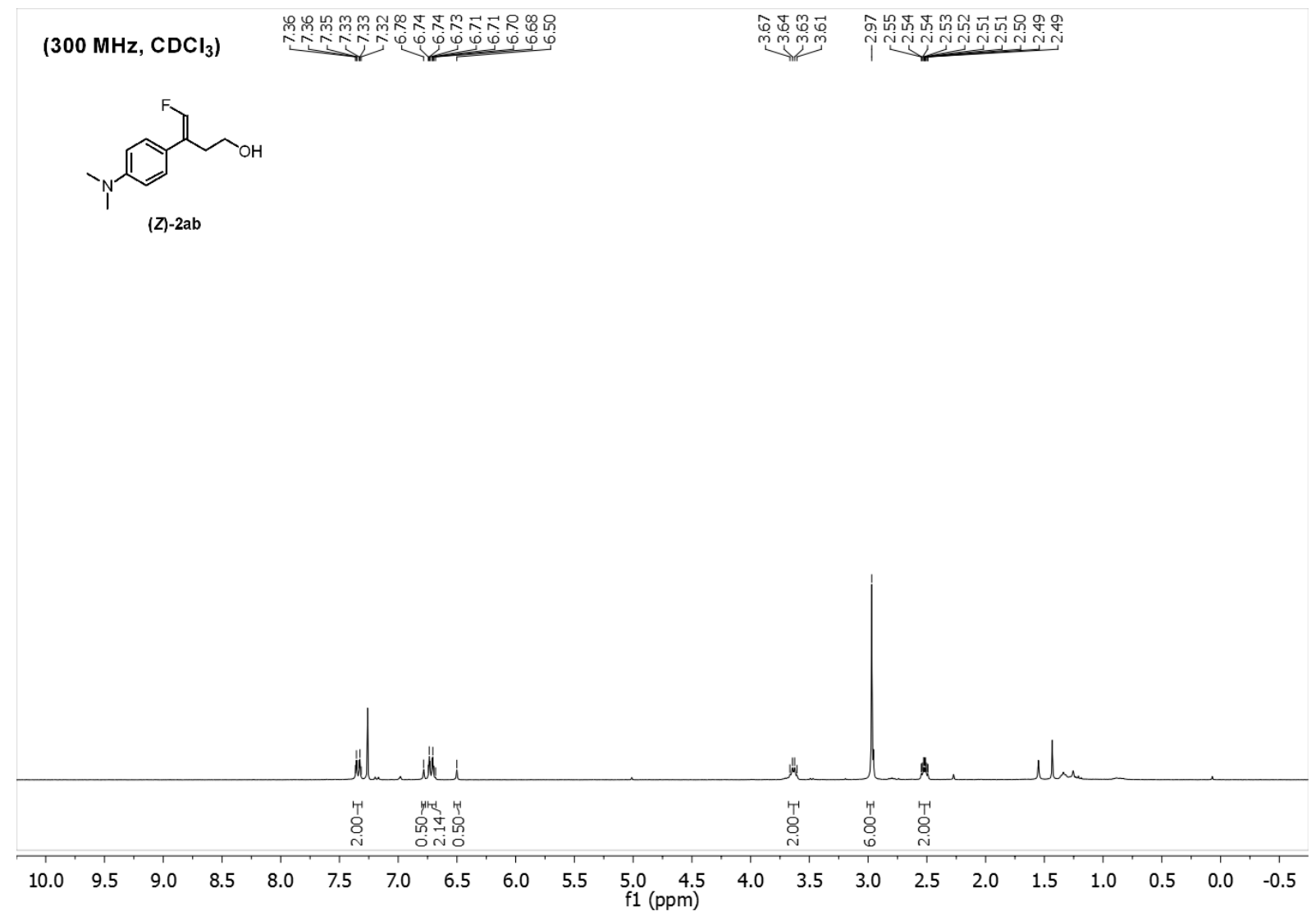

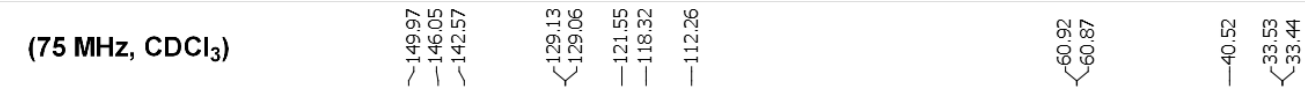

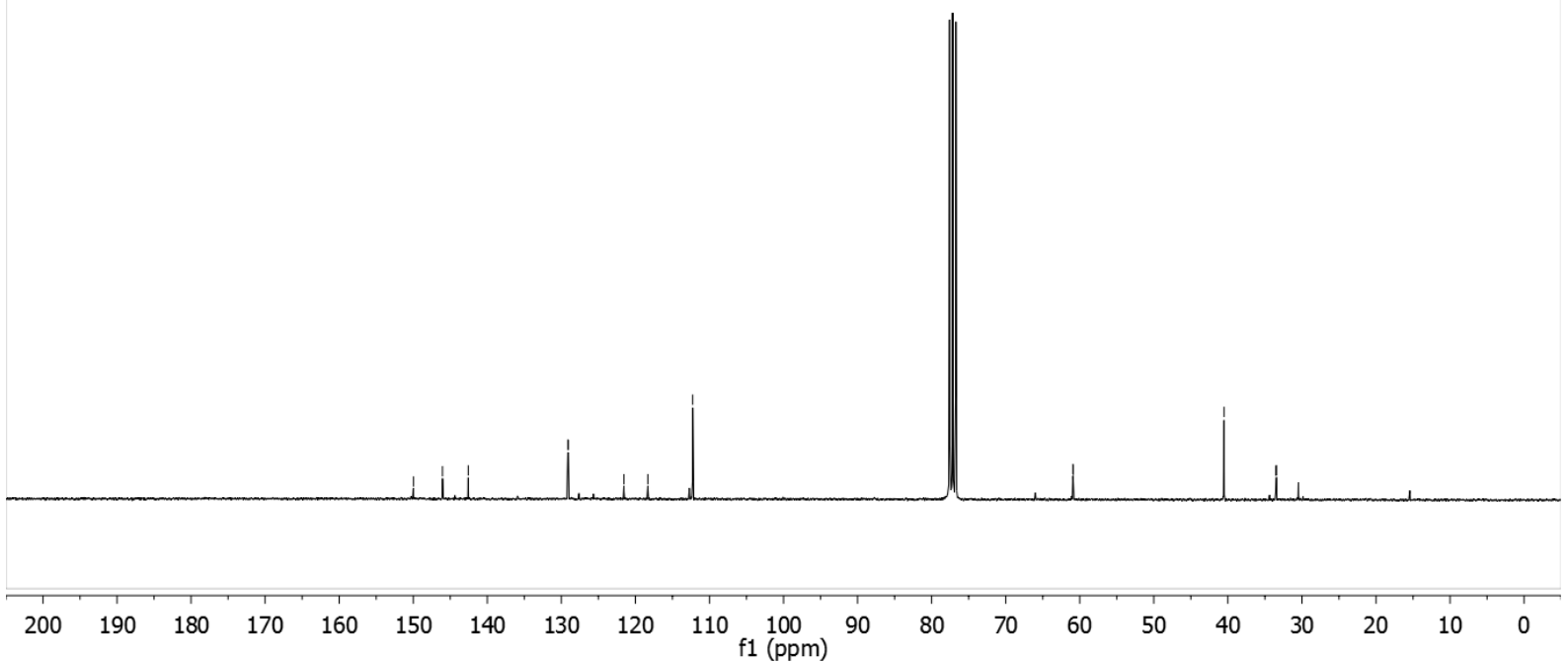


(282 $\mathrm{MHz}, \mathrm{CDCl}_{3}$ )

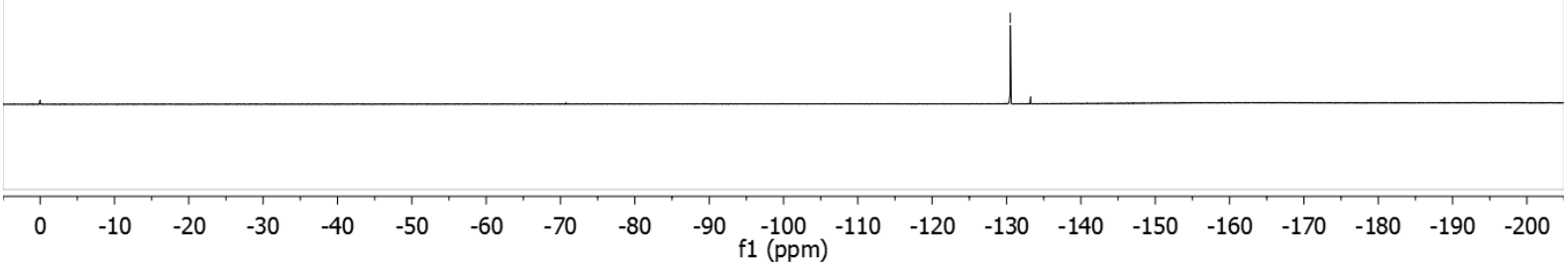

$\left(300 \mathrm{MHz}, \mathrm{CDCl}_{3}\right)$

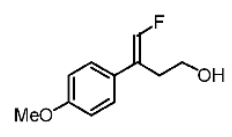

(E)-2ac

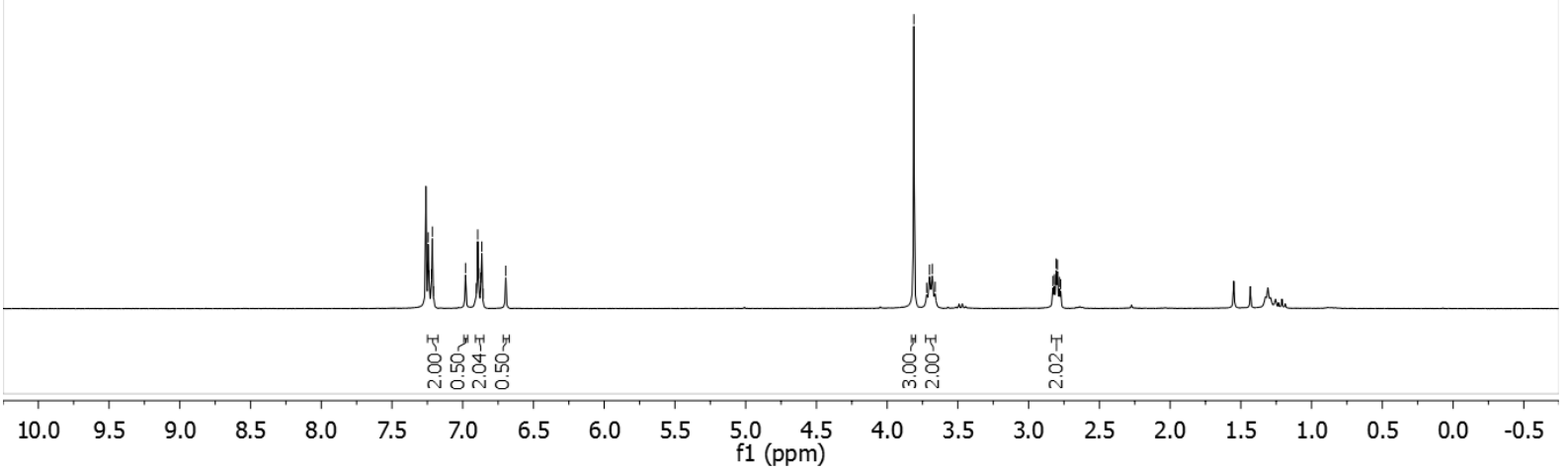




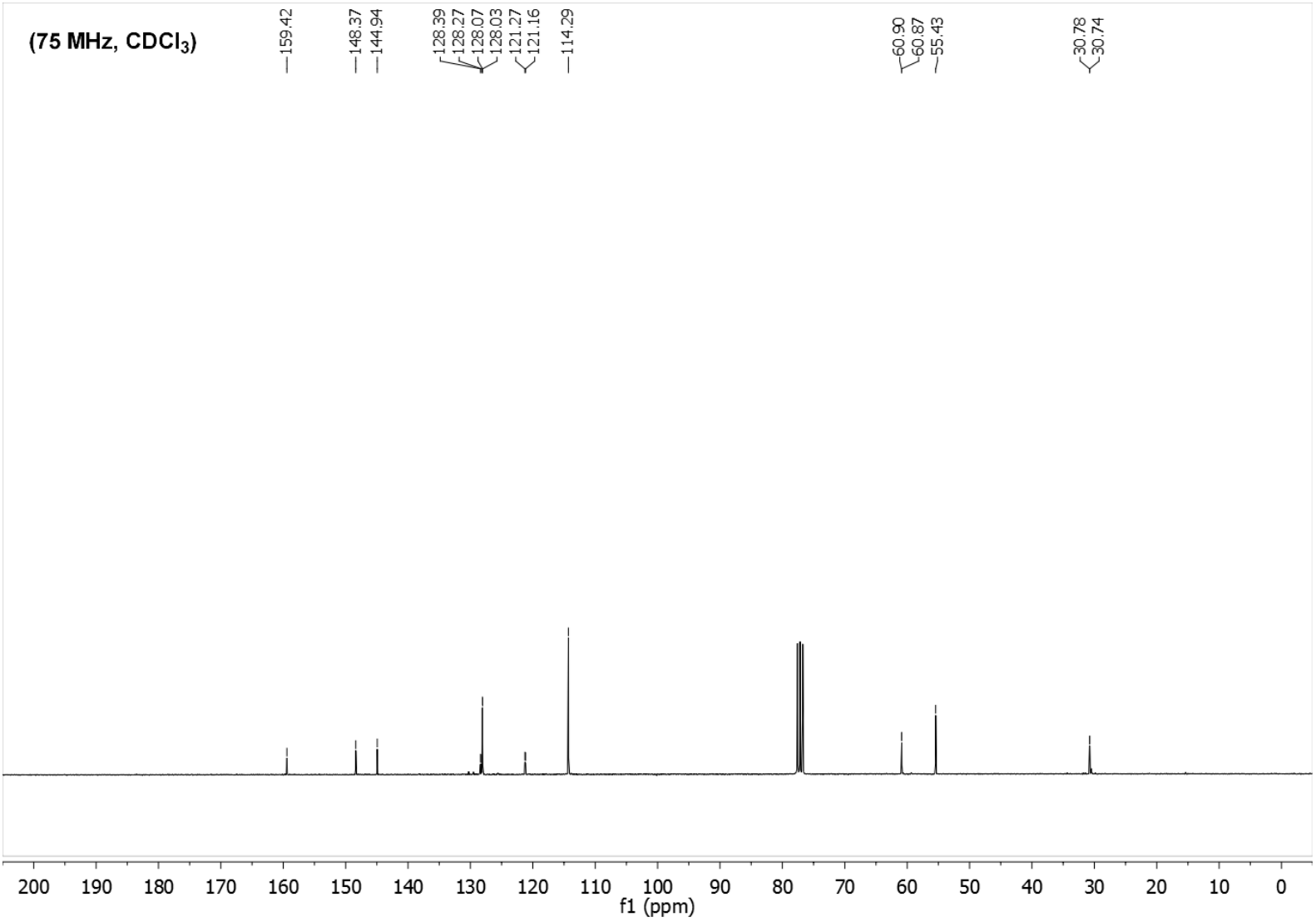

$\left(282 \mathrm{MHz}, \mathrm{CDCl}_{3}\right)$

\section{$\stackrel{\substack{m \\ \stackrel{m}{i}}}{1}$}

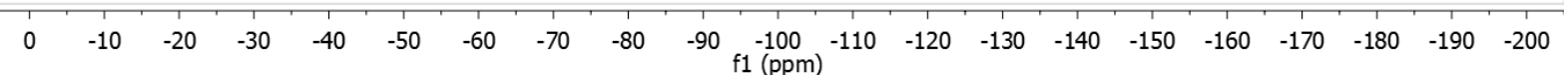




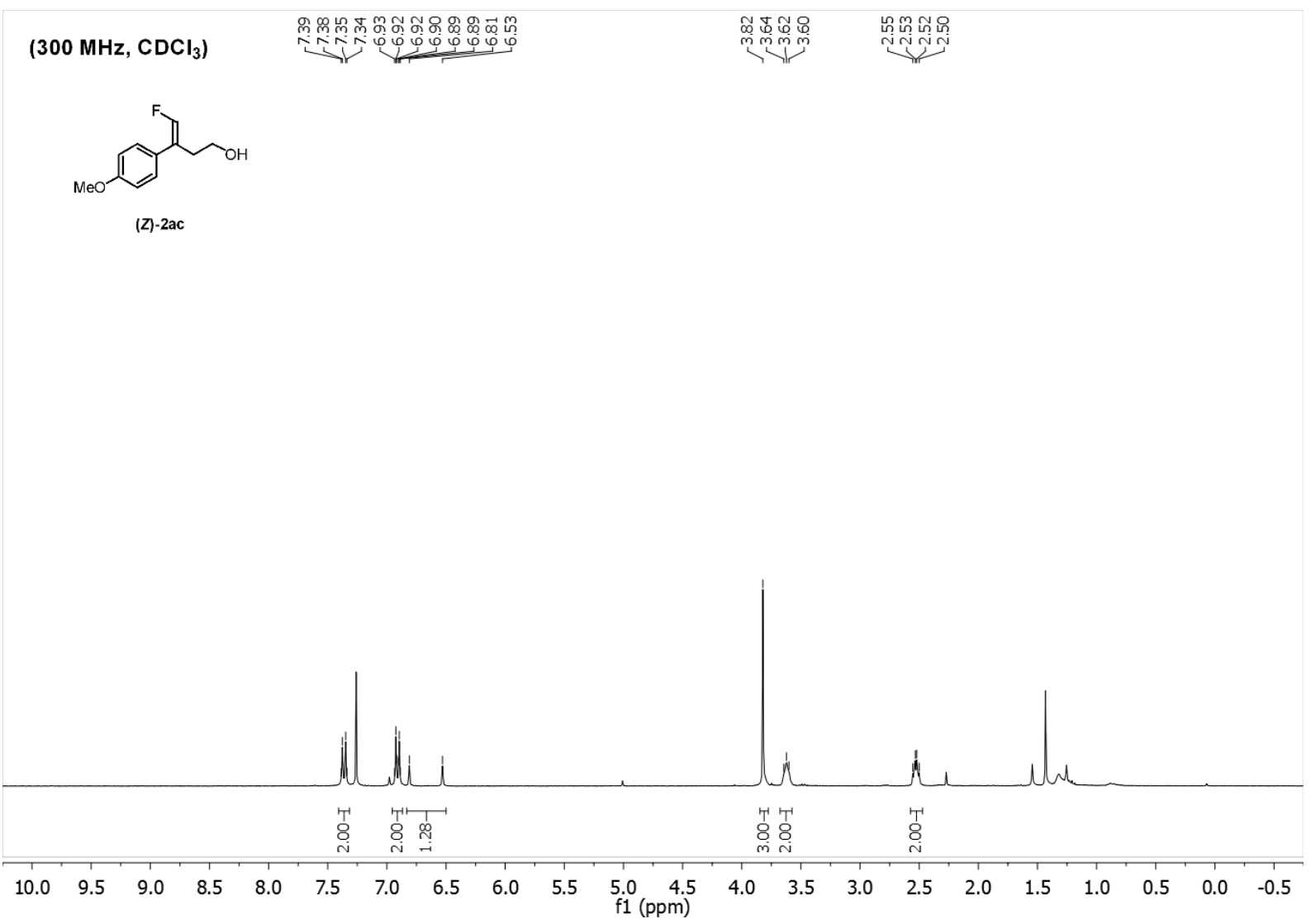

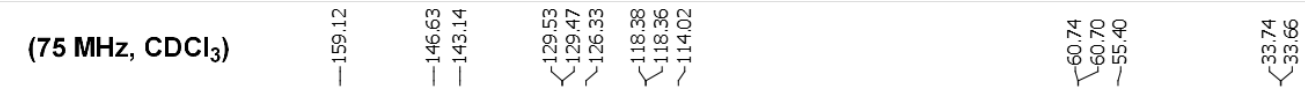

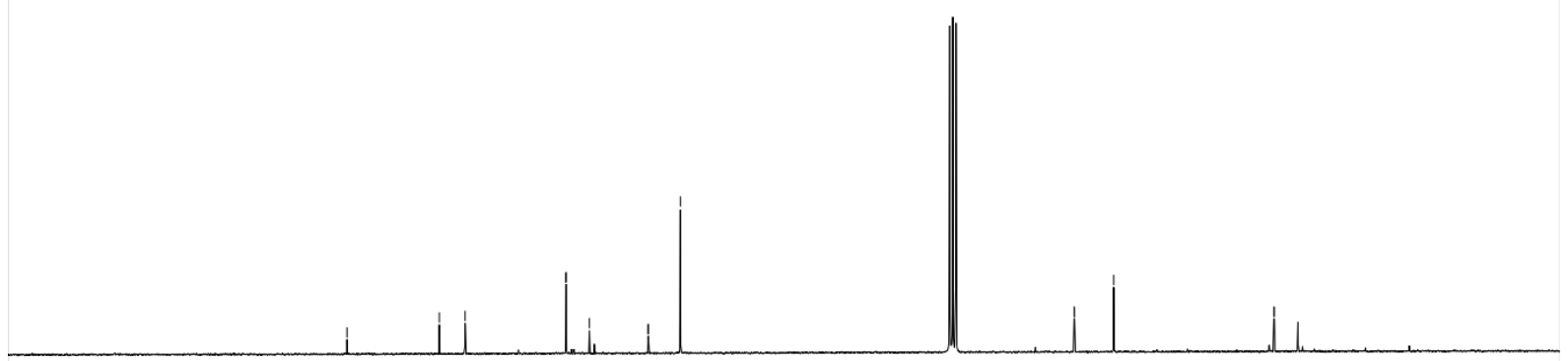

$\begin{array}{lllllllllllllllllllll}200 & 190 & 180 & 170 & 160 & 150 & 140 & 130 & 120 & 110 & 100 & 90 & 80 & 70 & 60 & 50 & 40 & 30 & 20 & 10 & 0\end{array}$ 
(282 MHz, $\mathrm{CDCl}_{3}$ )
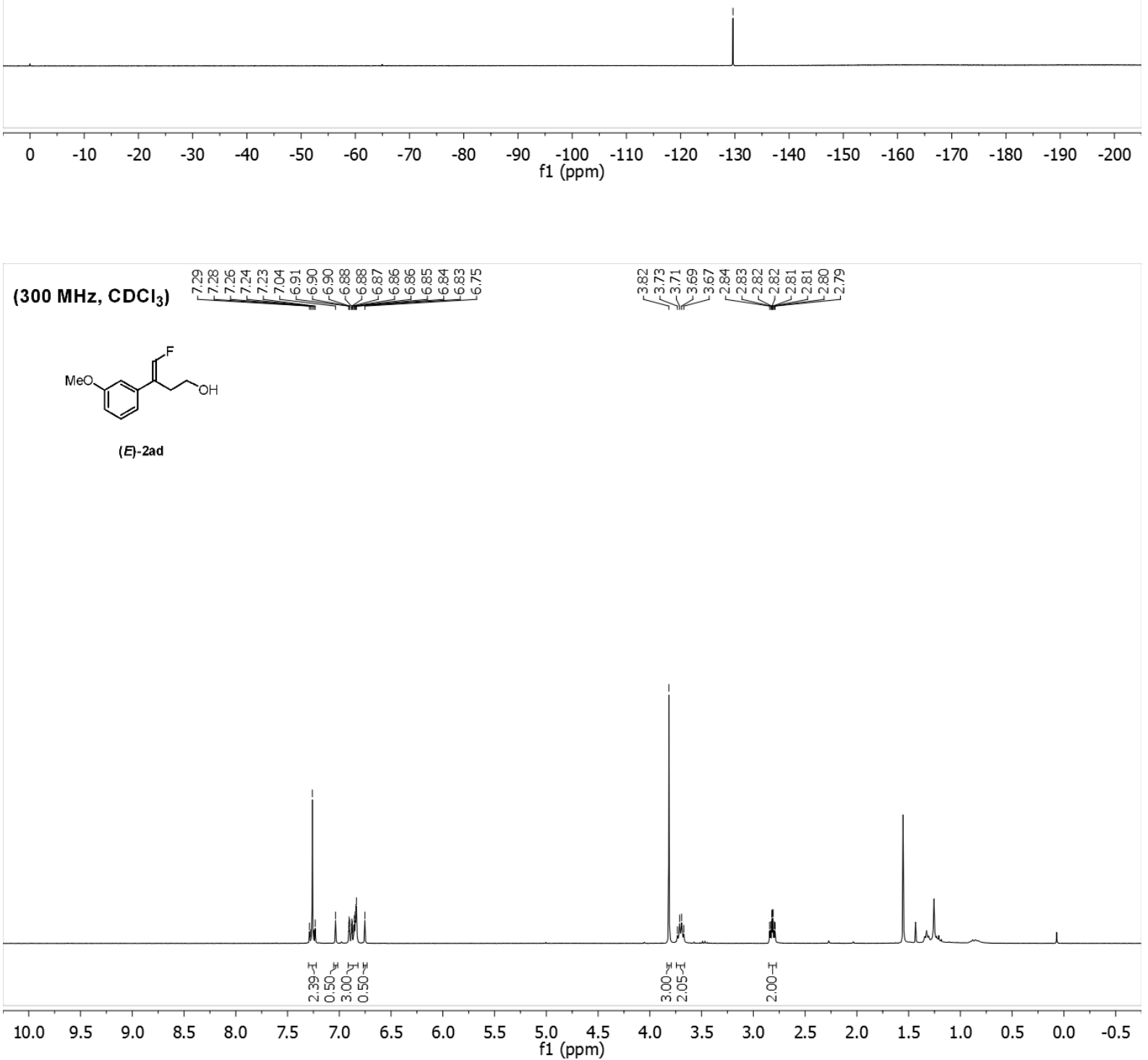


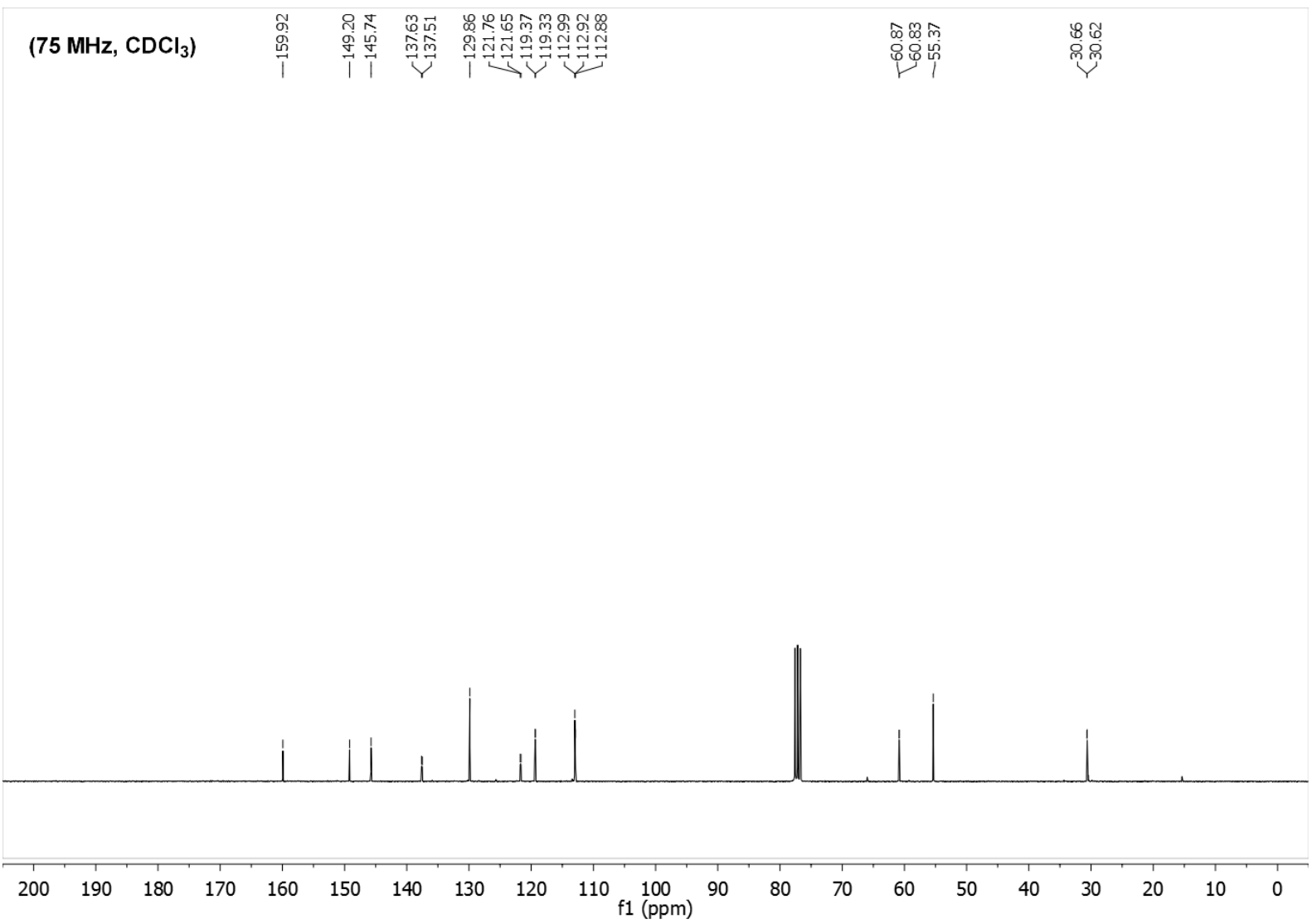

(282 $\mathrm{MHz}, \mathrm{CDCl}_{3}$ ) 


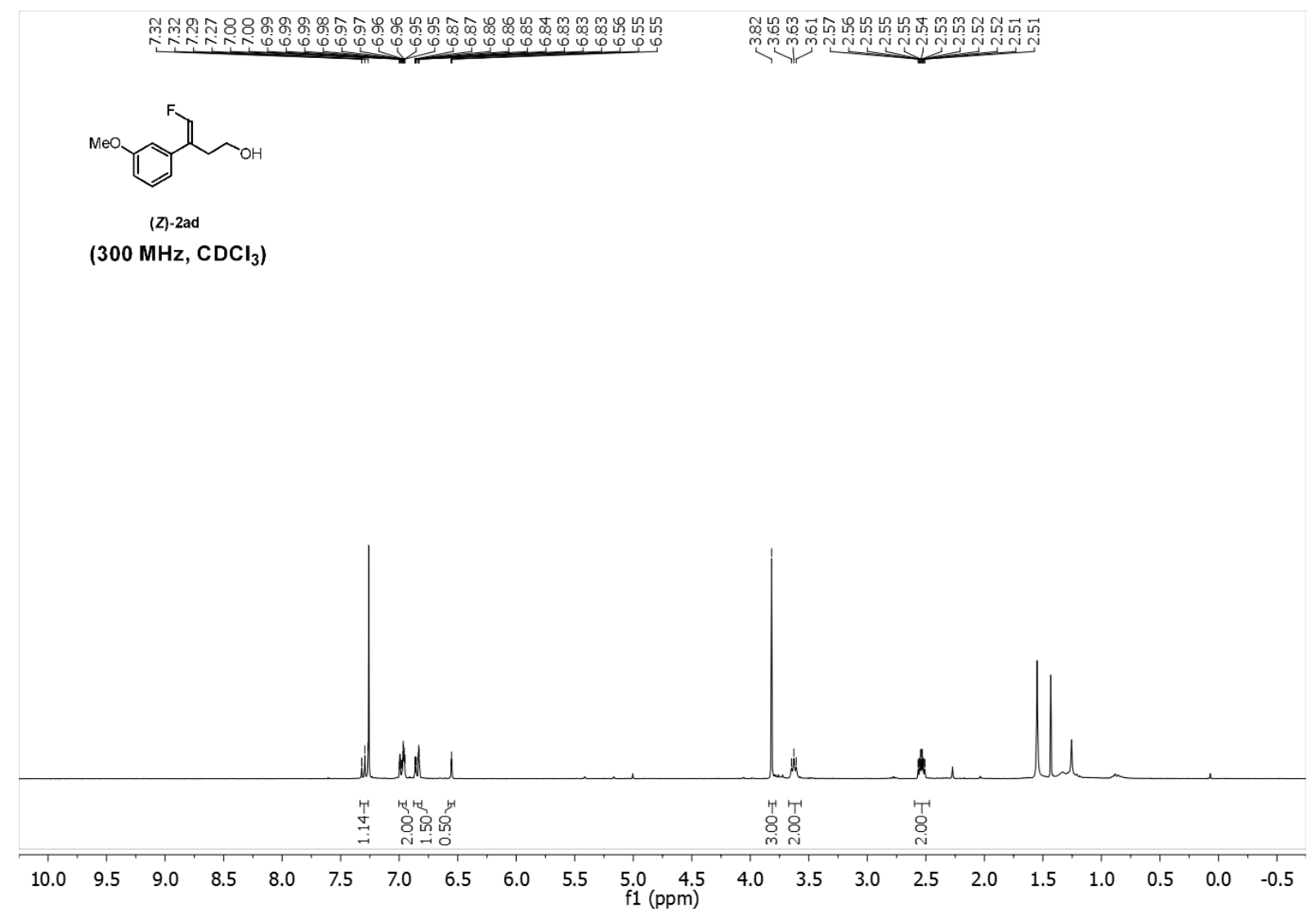

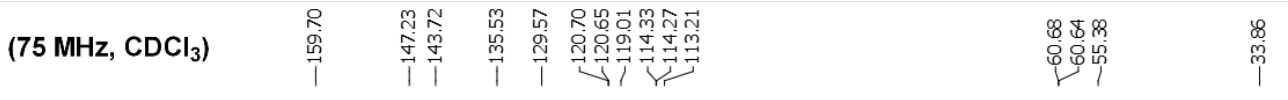

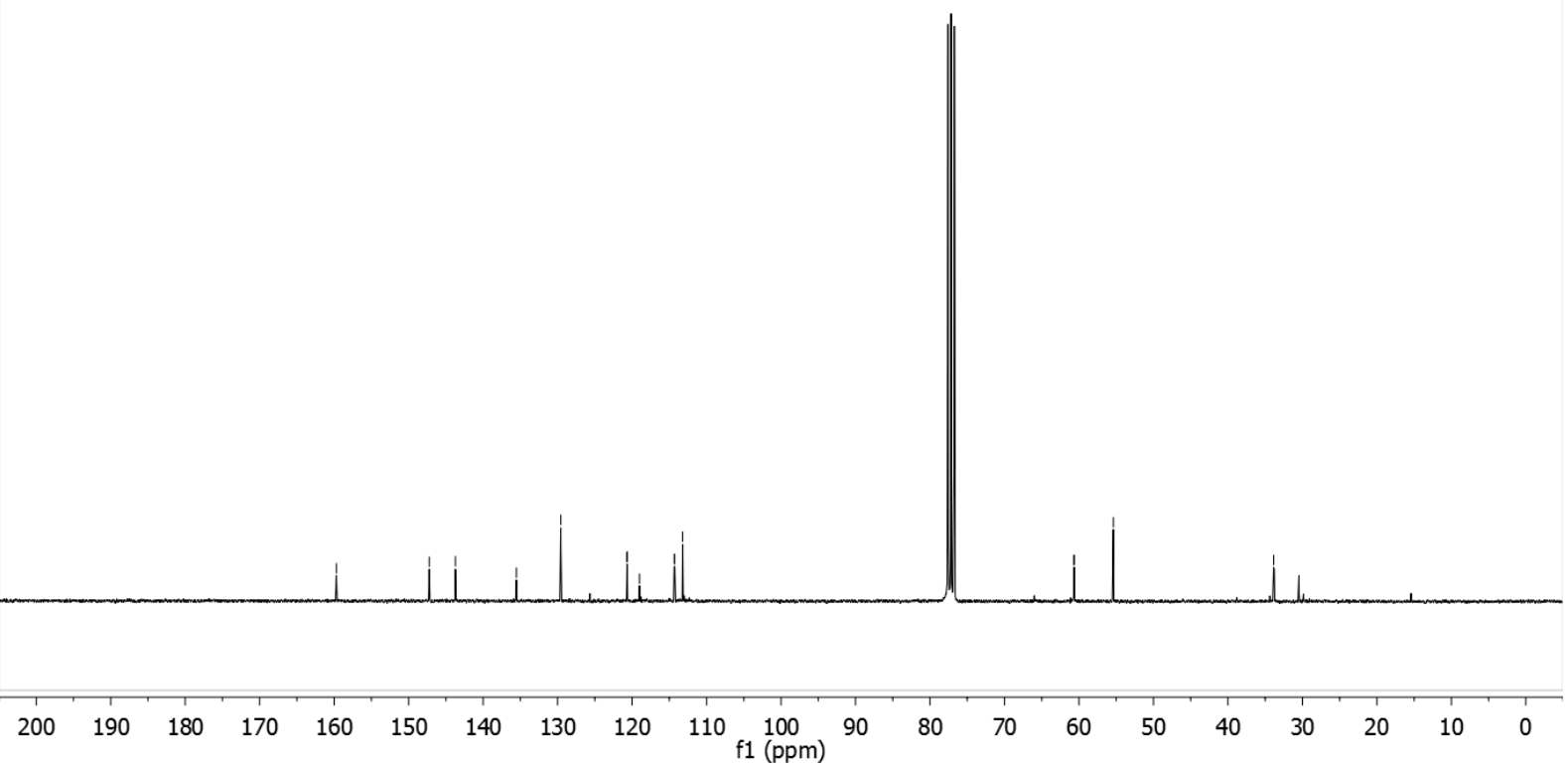


(282 MHz, $\mathrm{CDCl}_{3}$ )

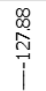
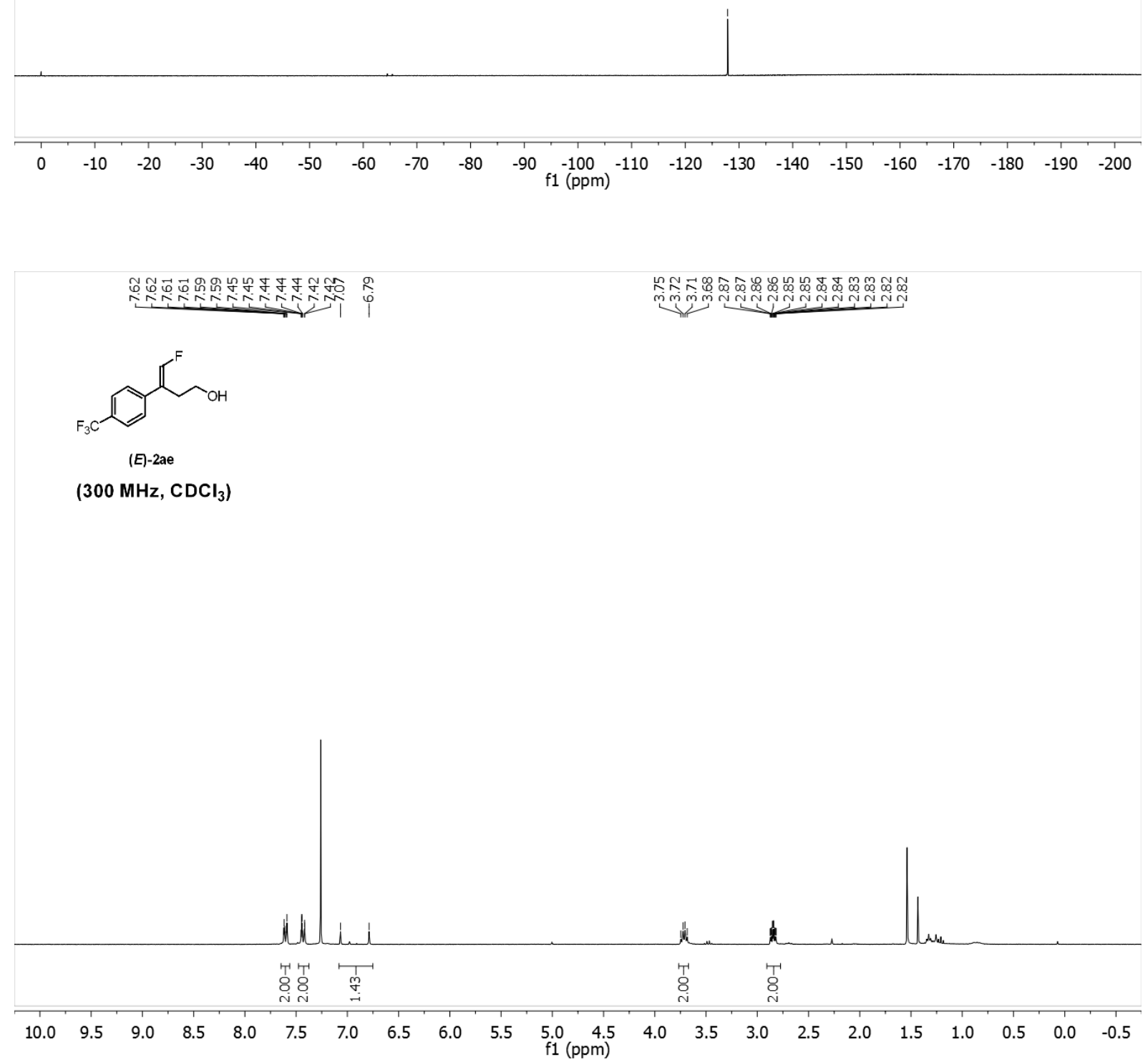


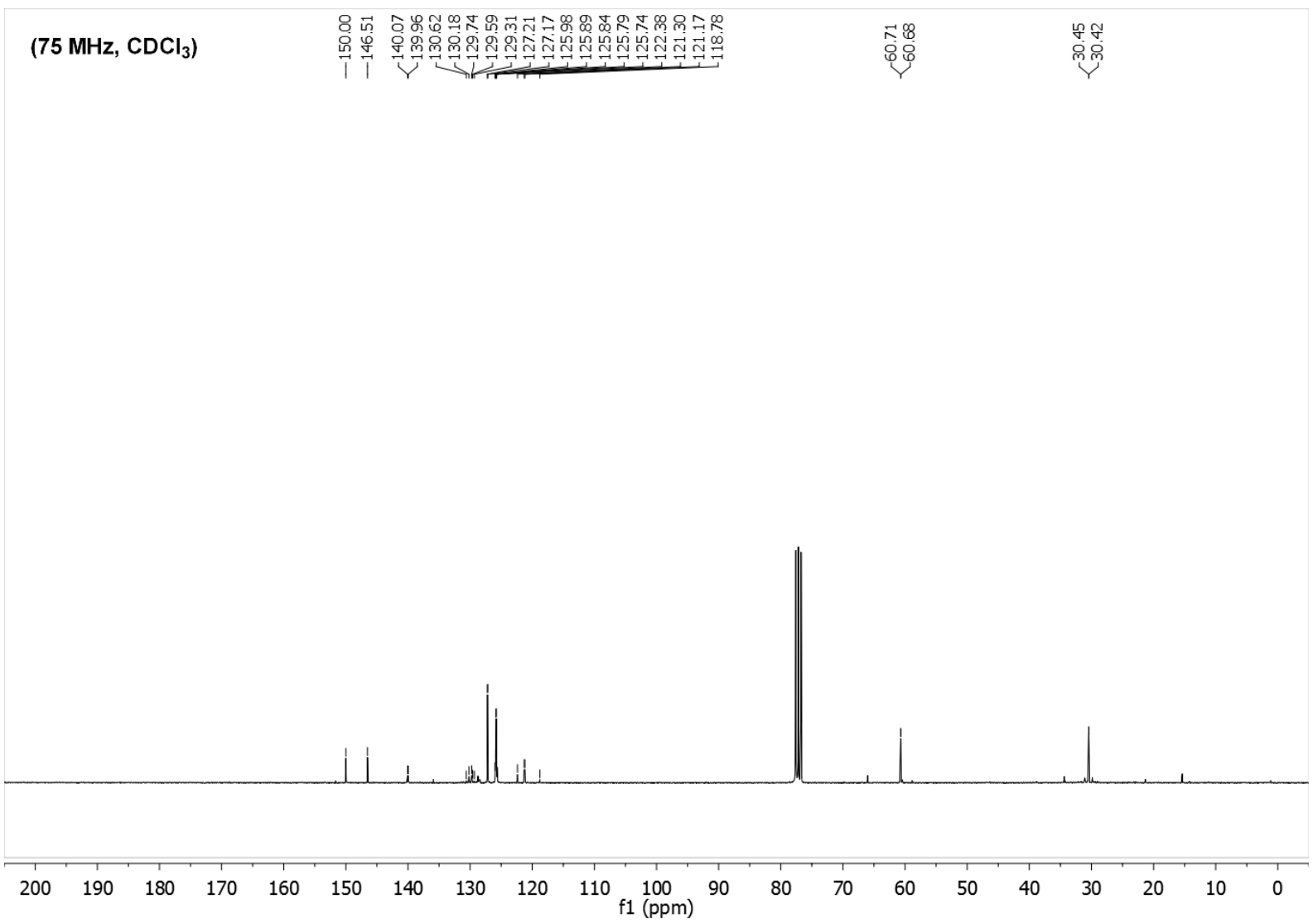

$\left(282 \mathrm{MHz}, \mathrm{CDCl}_{3}\right)$
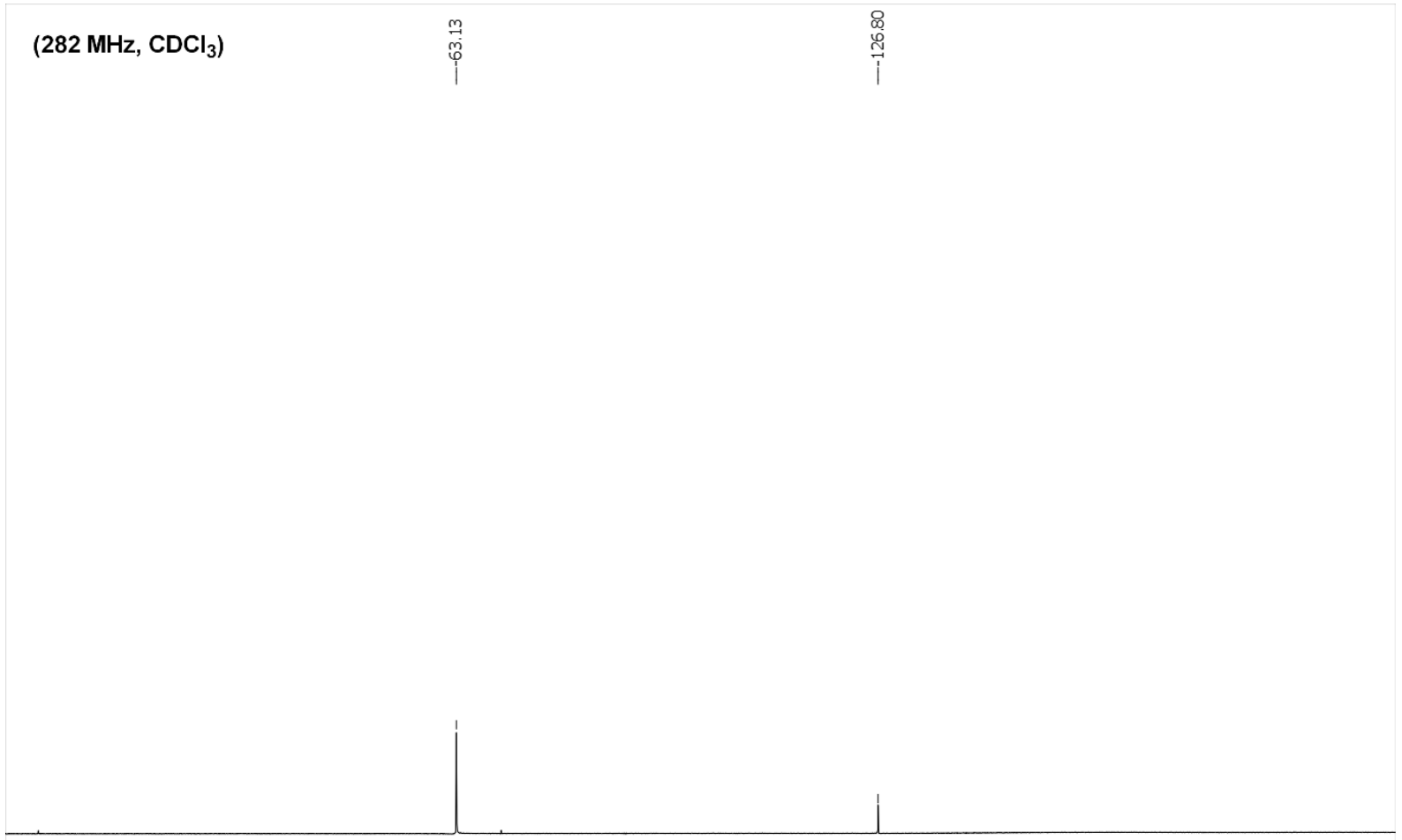

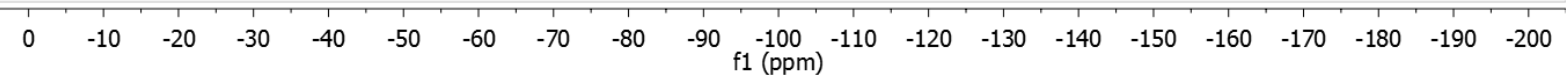




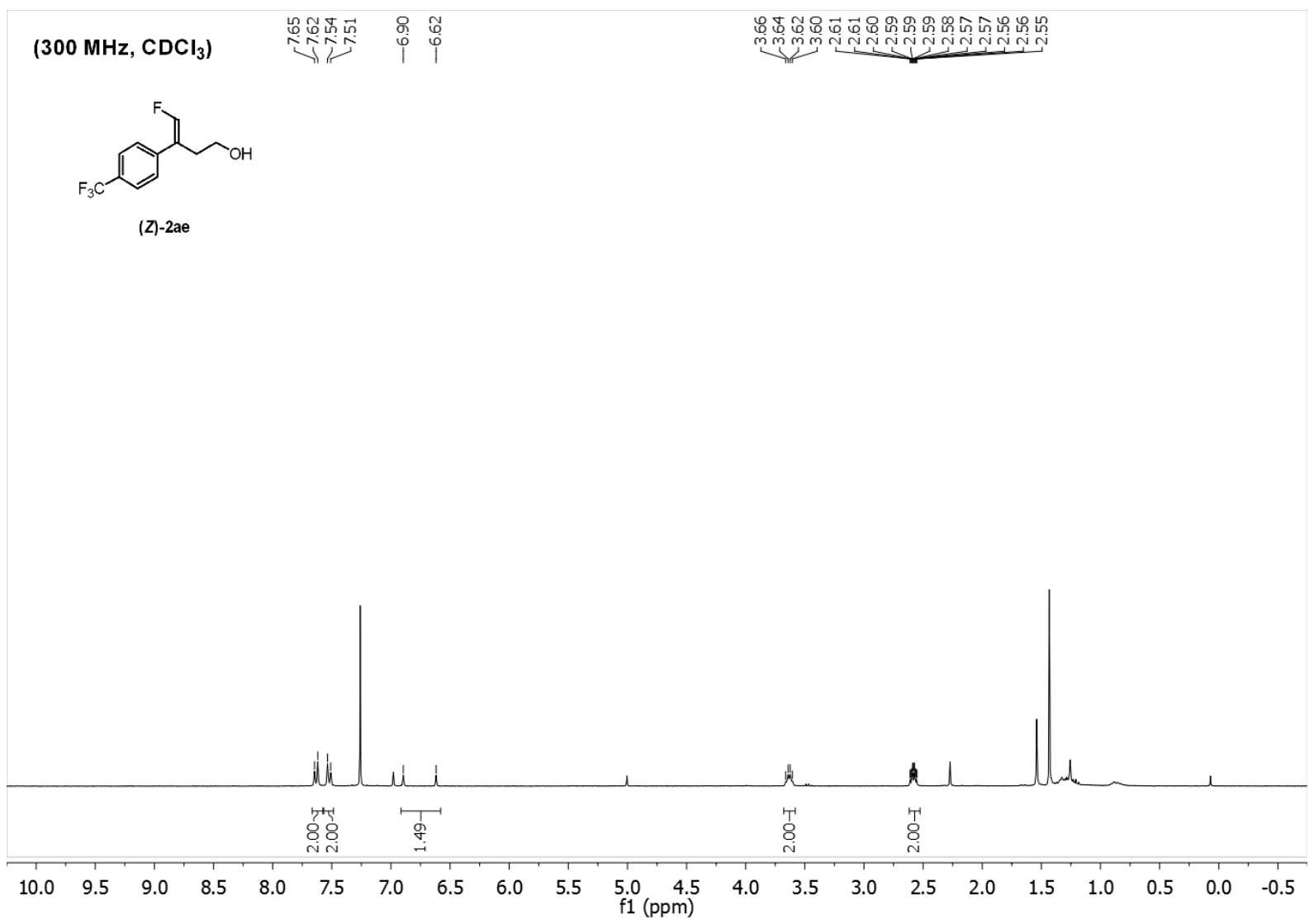

(75 $\mathrm{MHz}, \mathrm{CDCl}_{3}$ )

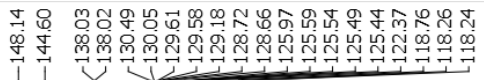

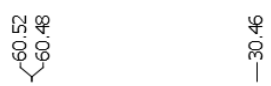

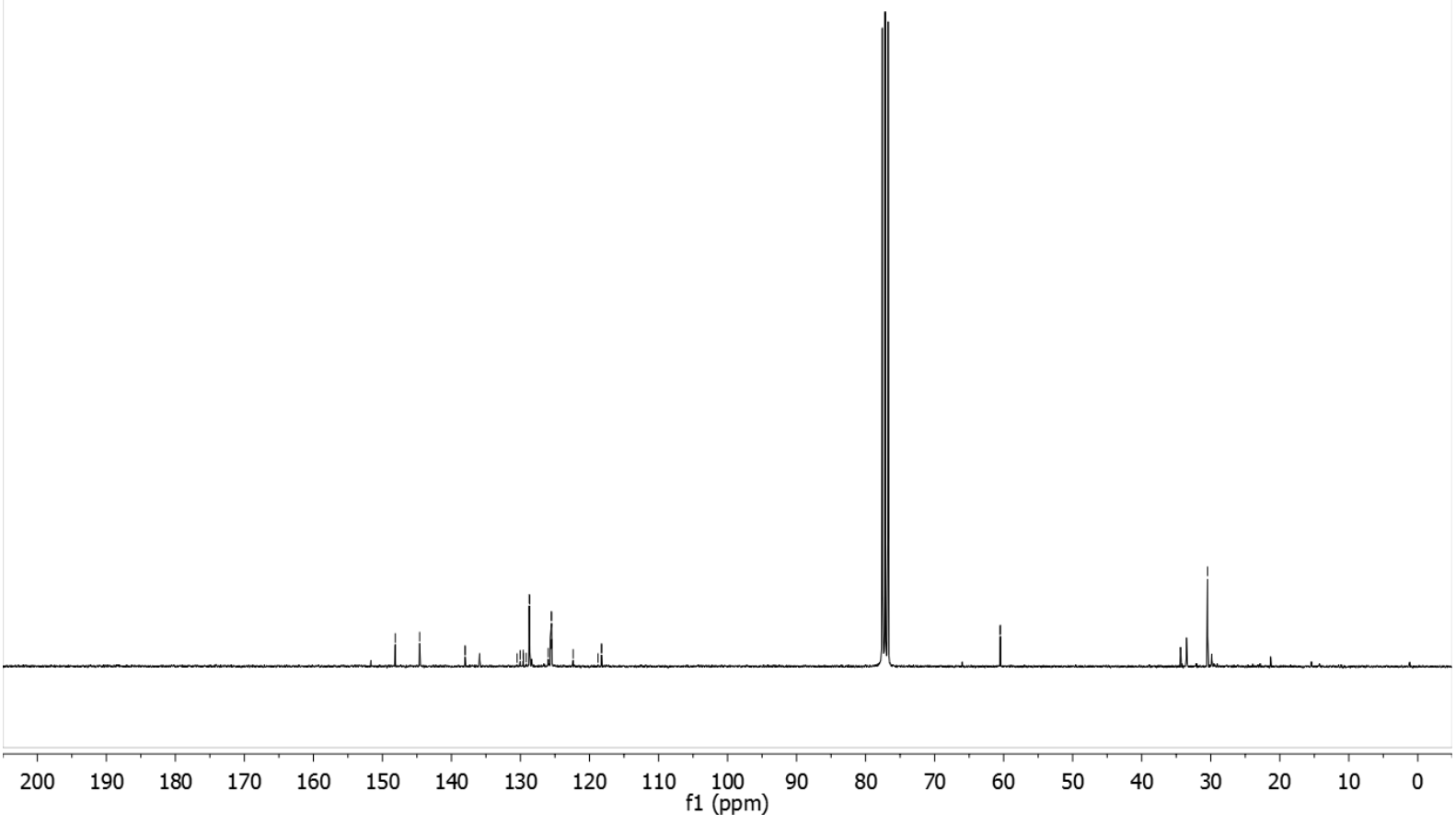


(282 MHz, $\mathrm{CDCl}_{3}$ )

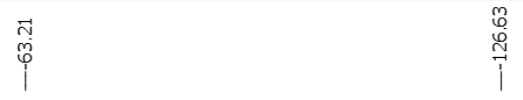

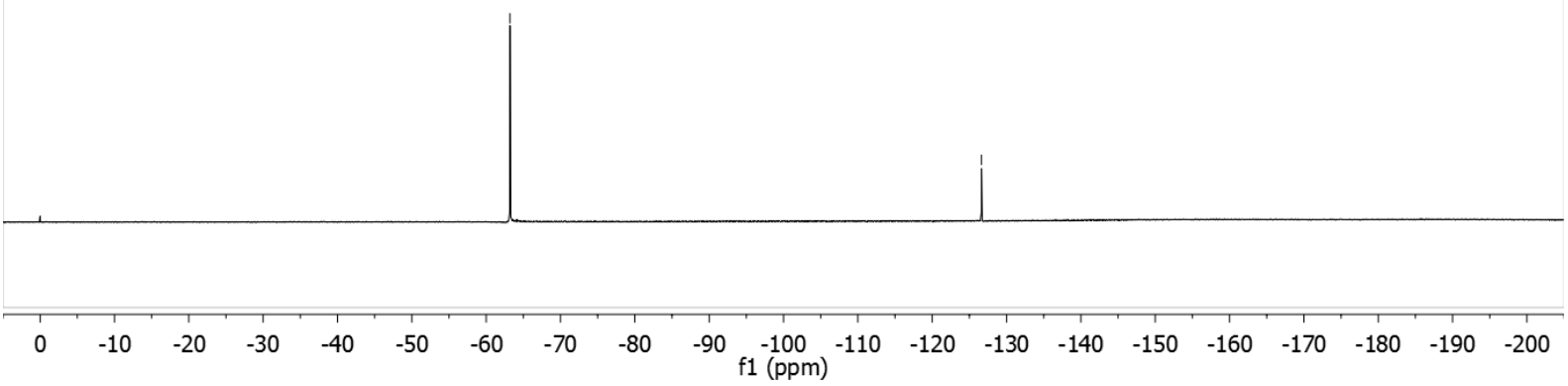

(300 MHz, $\left.\mathrm{CDCl}_{3}\right)$

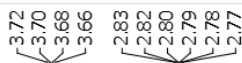<smiles>OCCC(=CCF)c1ccc(F)cc1</smiles>

(E)-2af

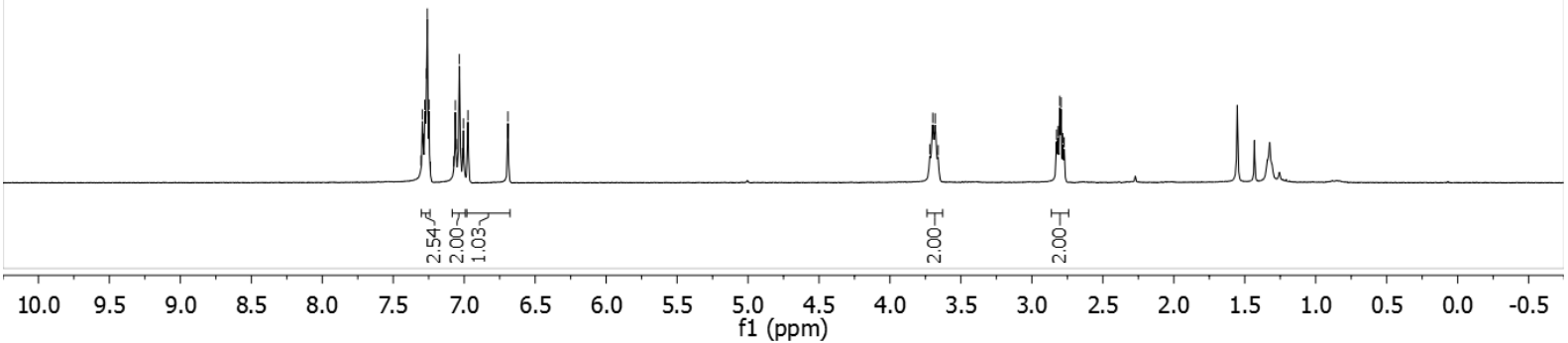




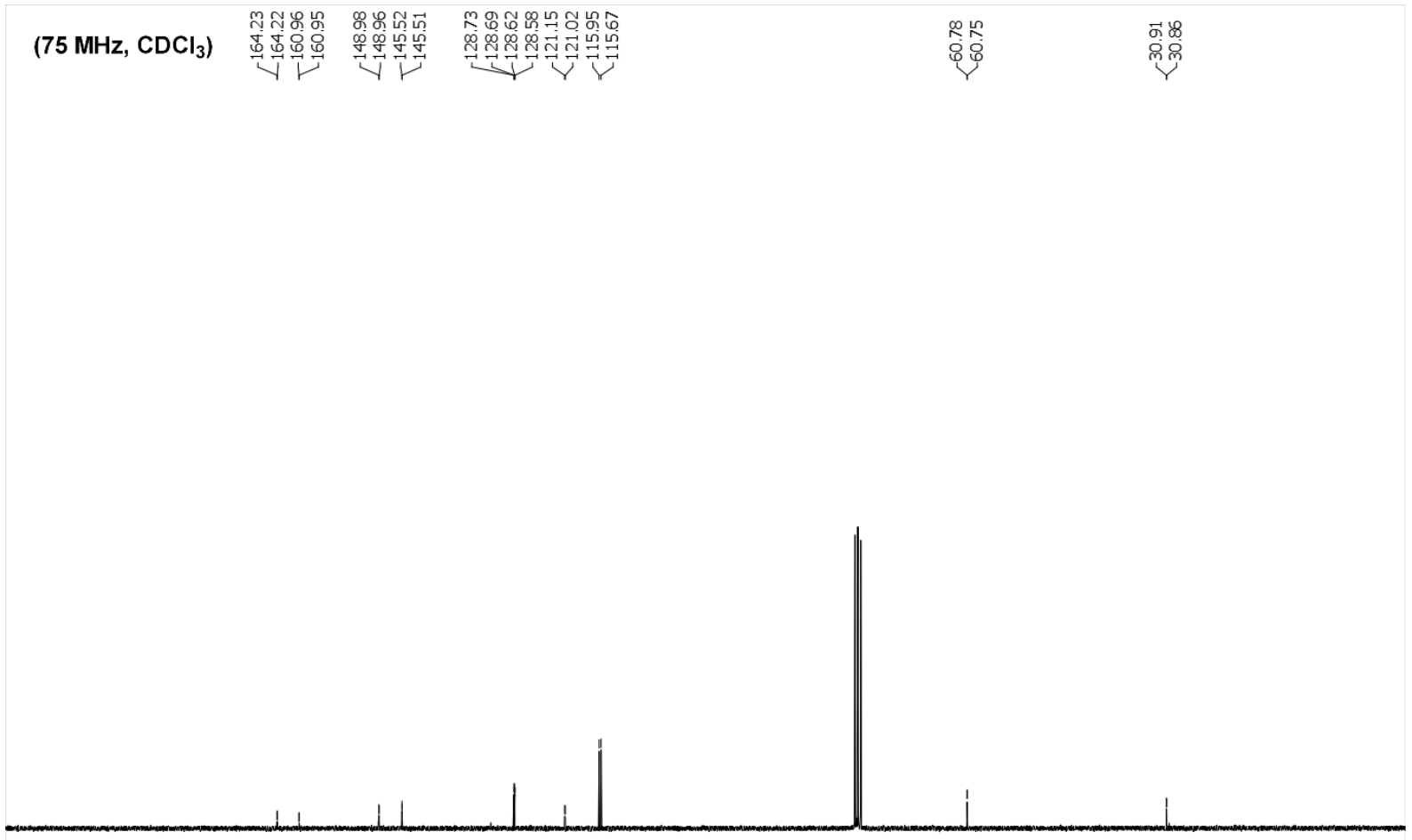

$\begin{array}{lllllllllllllllllllllllllllll}200 & 190 & 180 & 170 & 160 & 150 & 140 & 130 & 120 & 110 & 100 & 90 & 80 & 70 & 60 & 50 & 40 & 30 & 20 & 10 & 0\end{array}$

$\left(282 \mathrm{MHz}, \mathrm{CDCl}_{3}\right)$

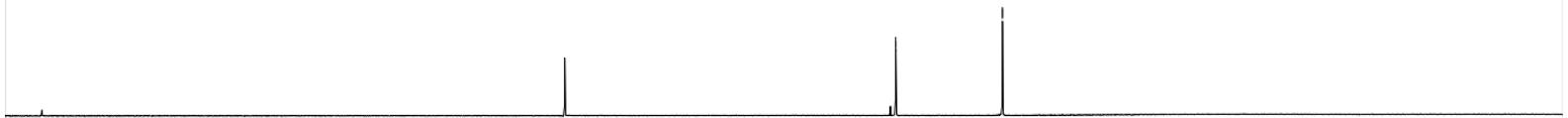

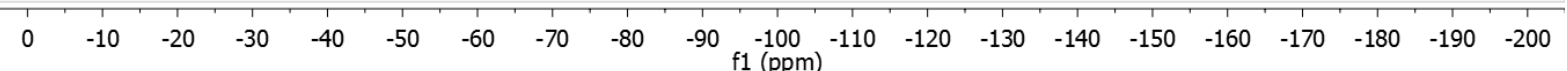




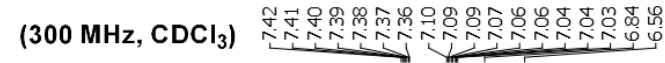<smiles>OCC/C(=C/F)c1ccc(F)cc1</smiles>

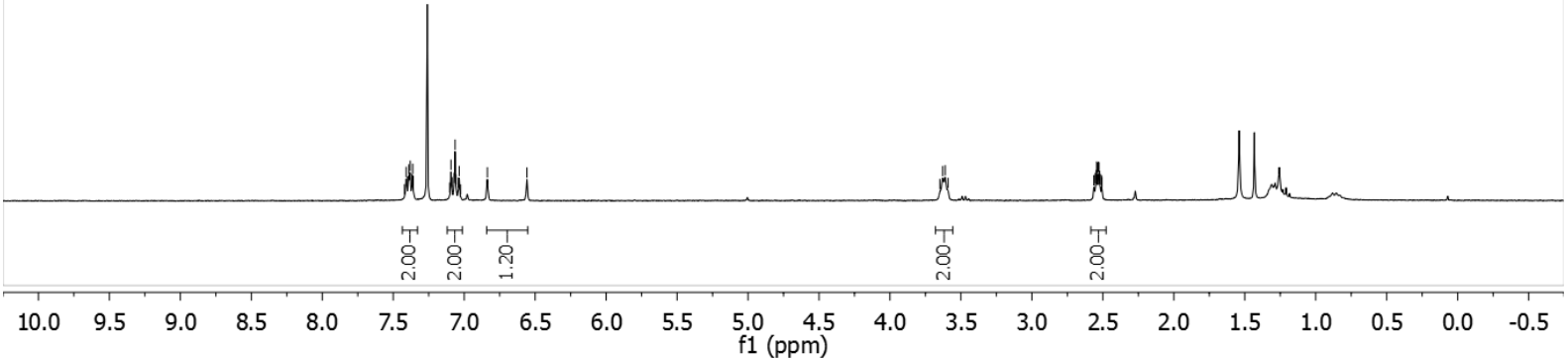

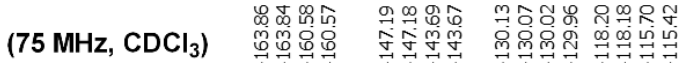

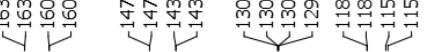

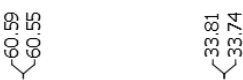

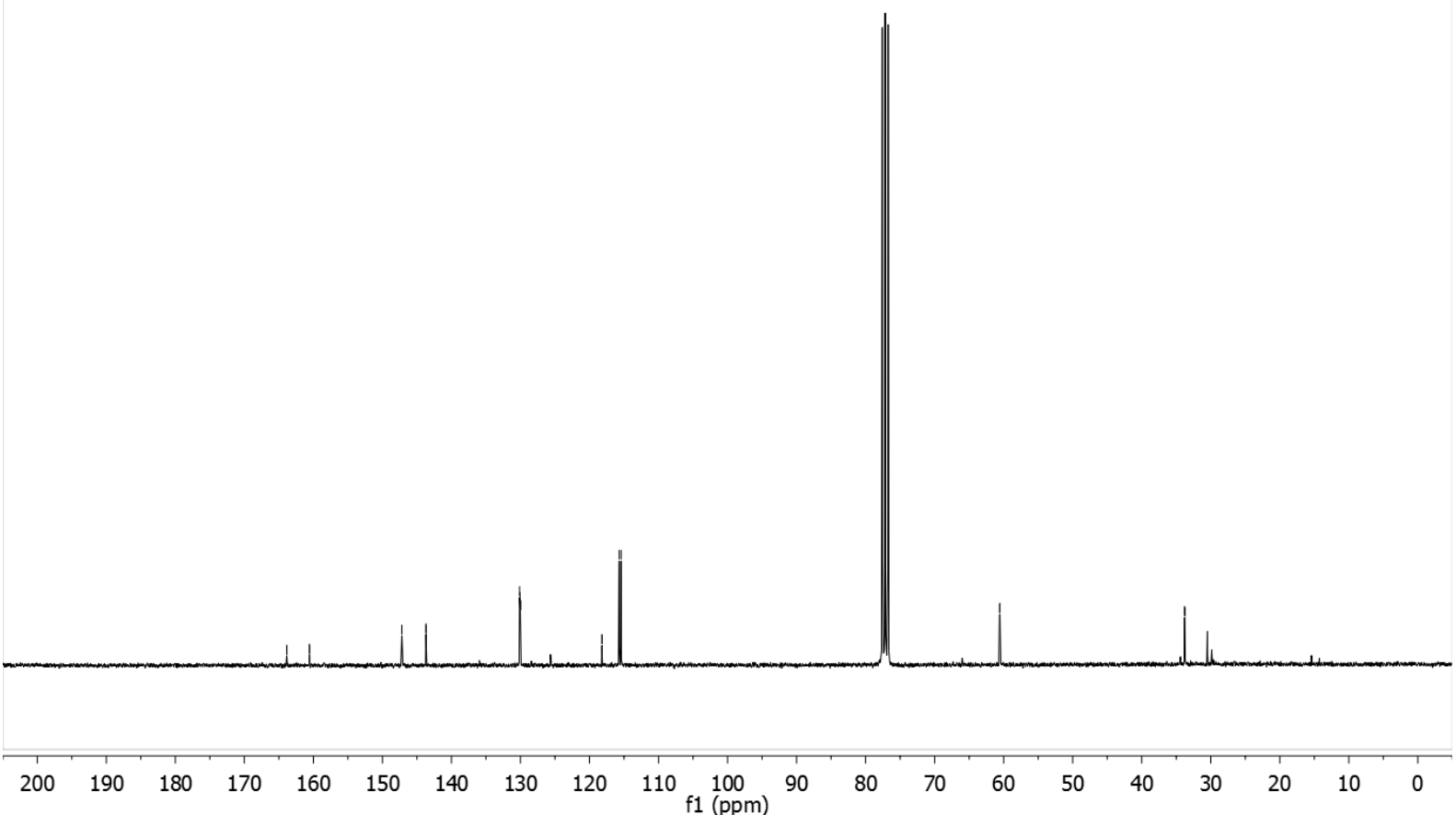


(282 $\mathrm{MHz}, \mathrm{CDCl}_{3}$ )

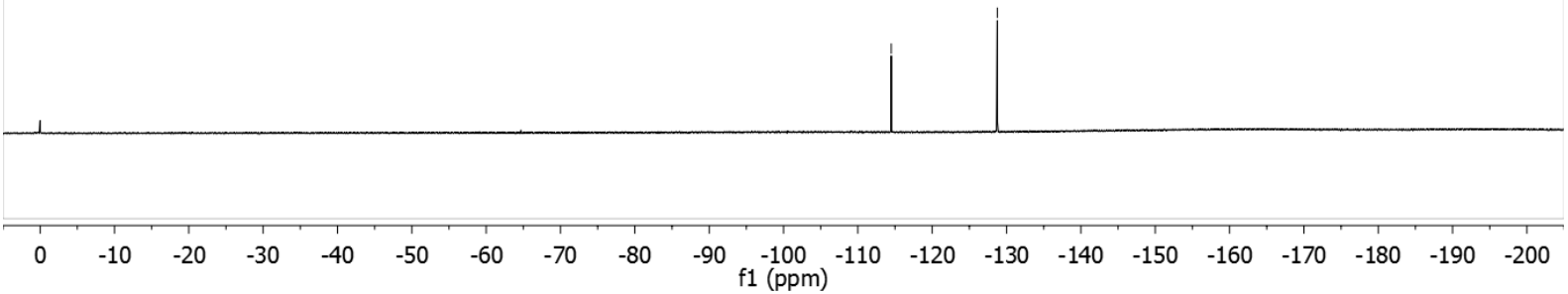

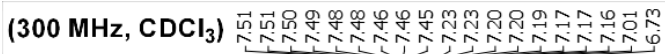

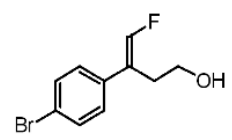

(E)-2ag

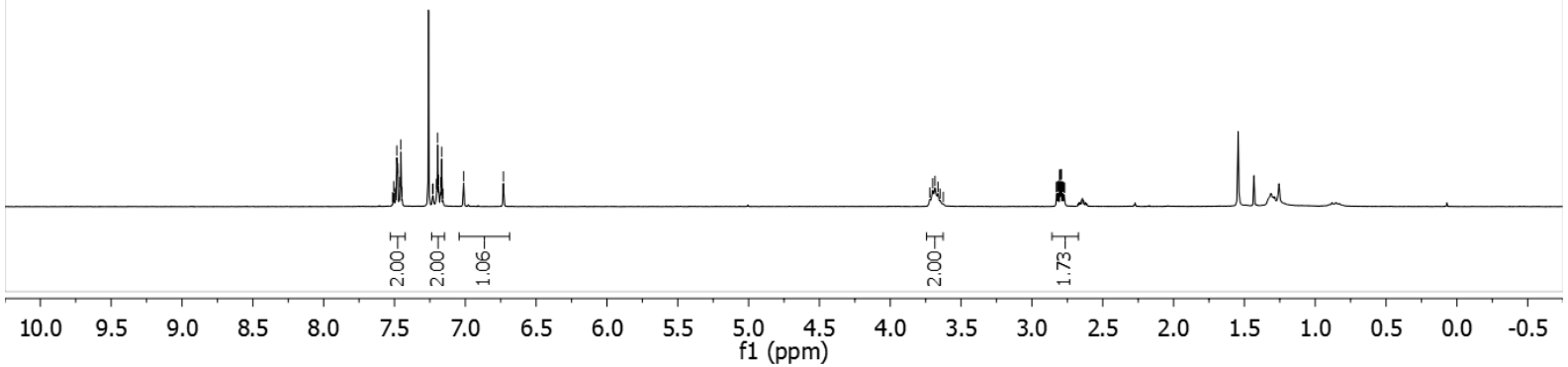




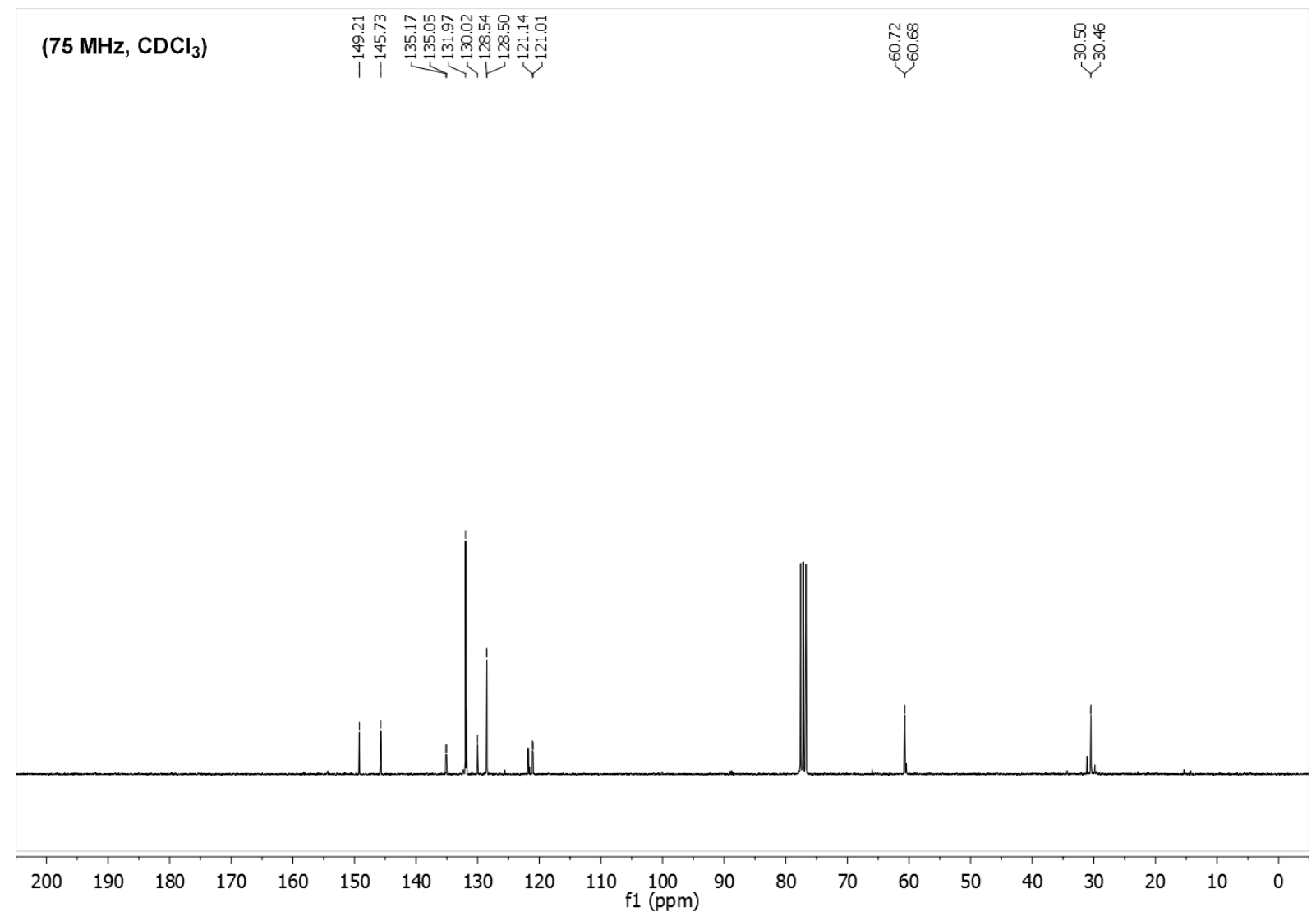

(282 MHz, $\mathrm{CDCl}_{3}$ )
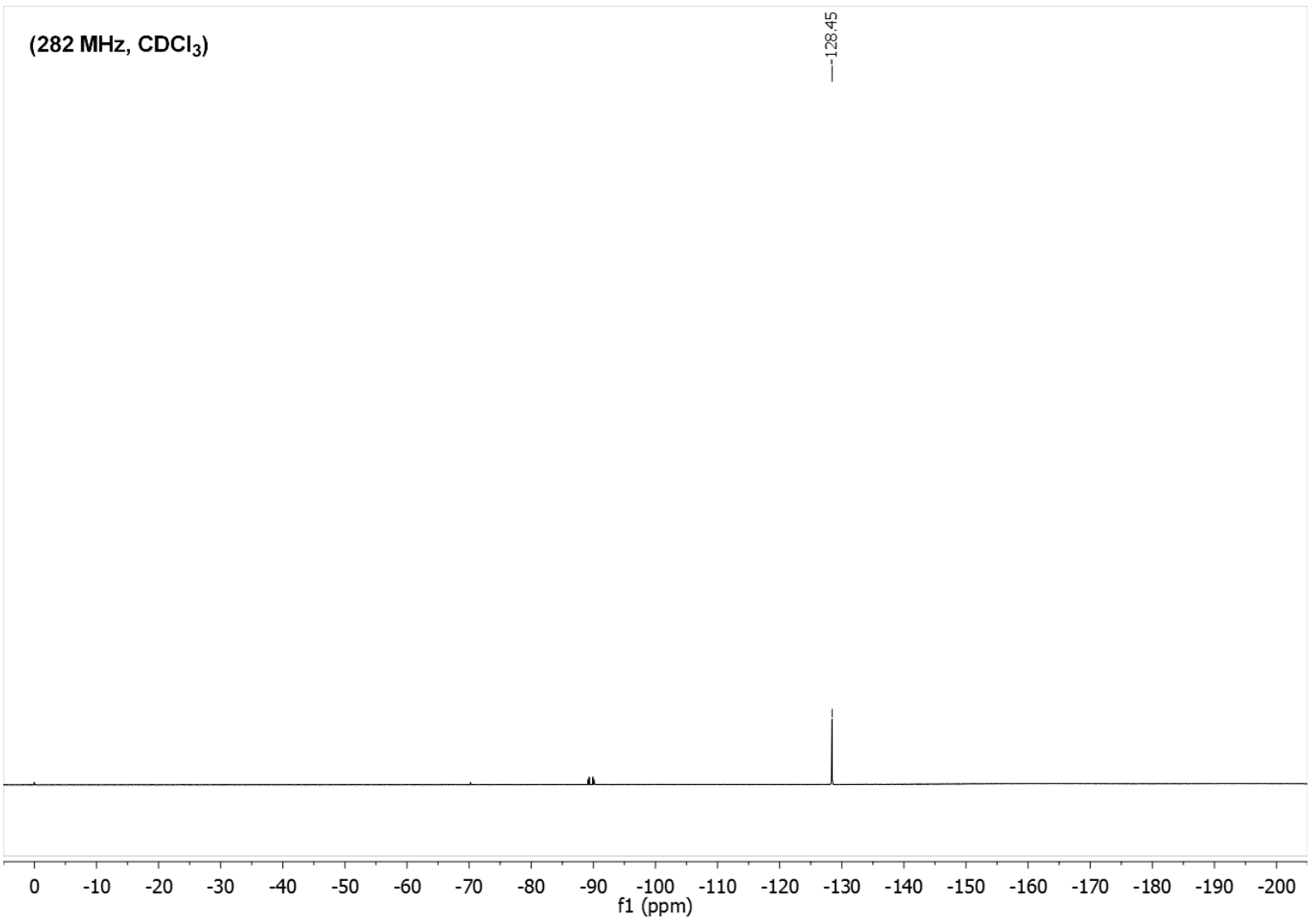


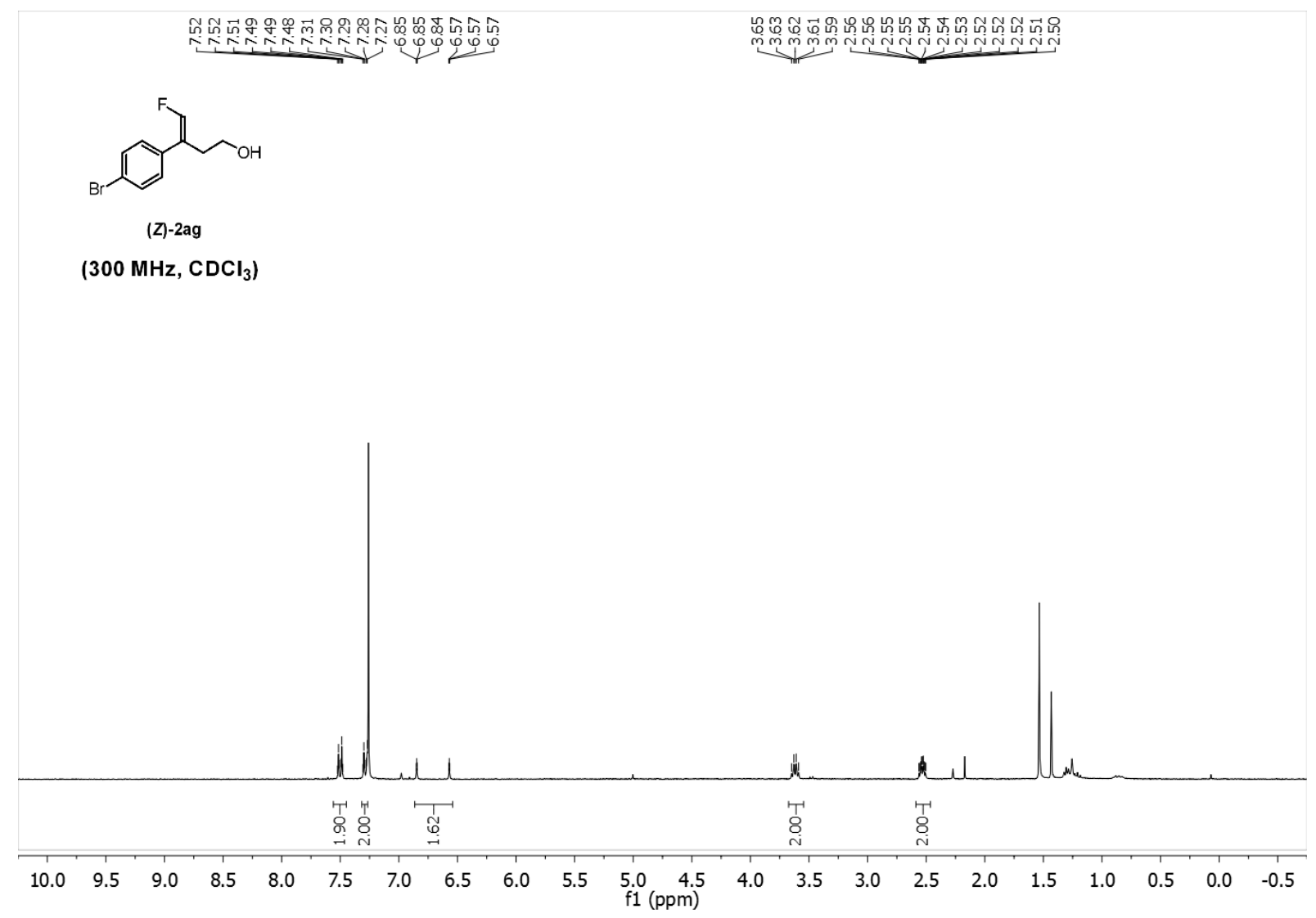

(75 MHz, $\mathrm{CDCl}_{3}$ )

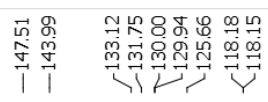

ฟ

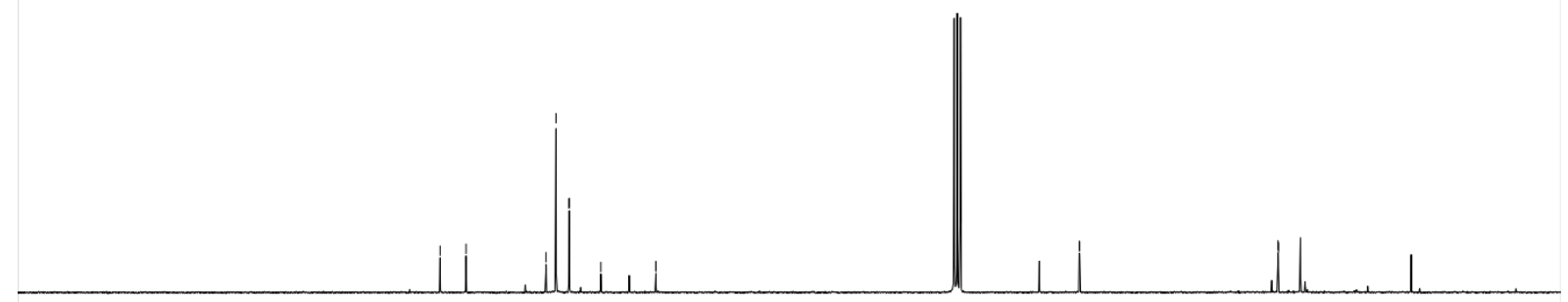

(300 $\mathrm{MHz}, \mathrm{CDCl}_{3}$ ) 
(282 $\mathrm{MHz}, \mathrm{CDCl}_{3}$ )

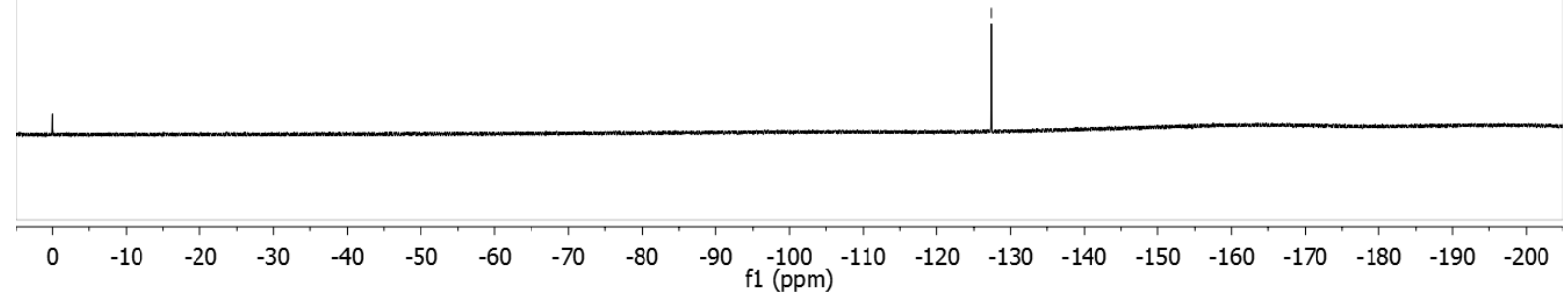

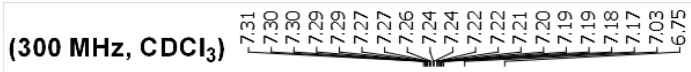
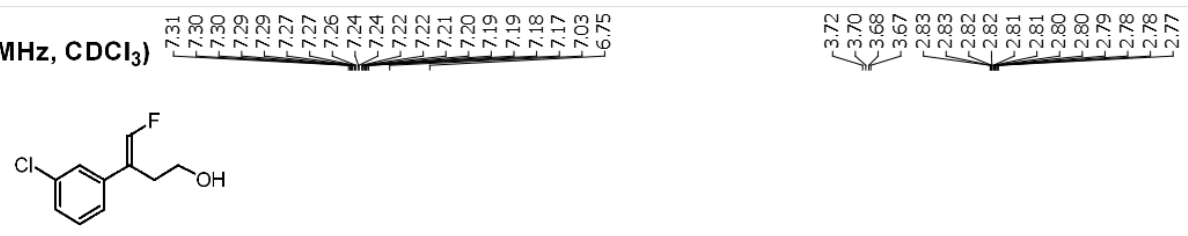

(E)-2ah

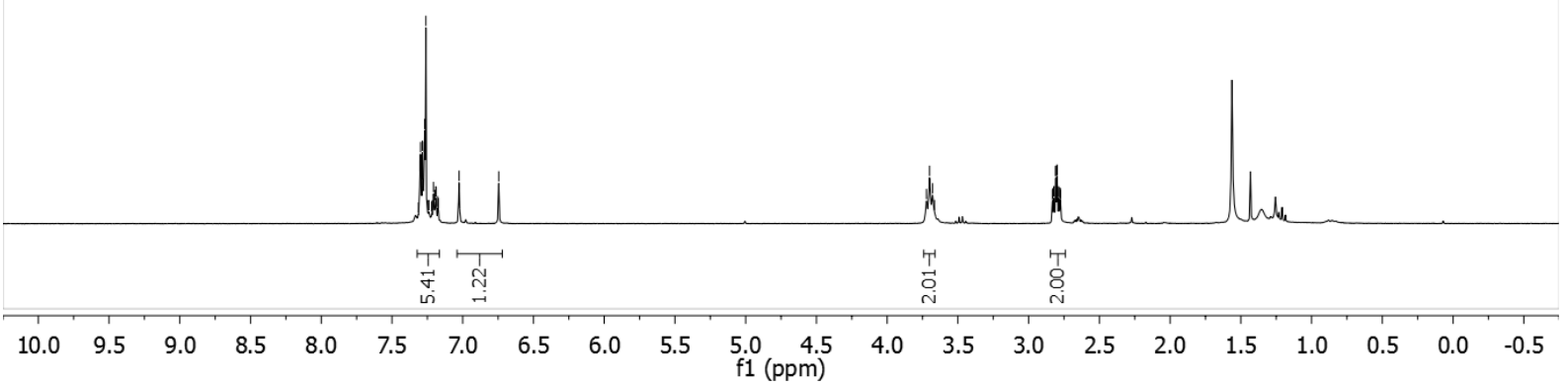




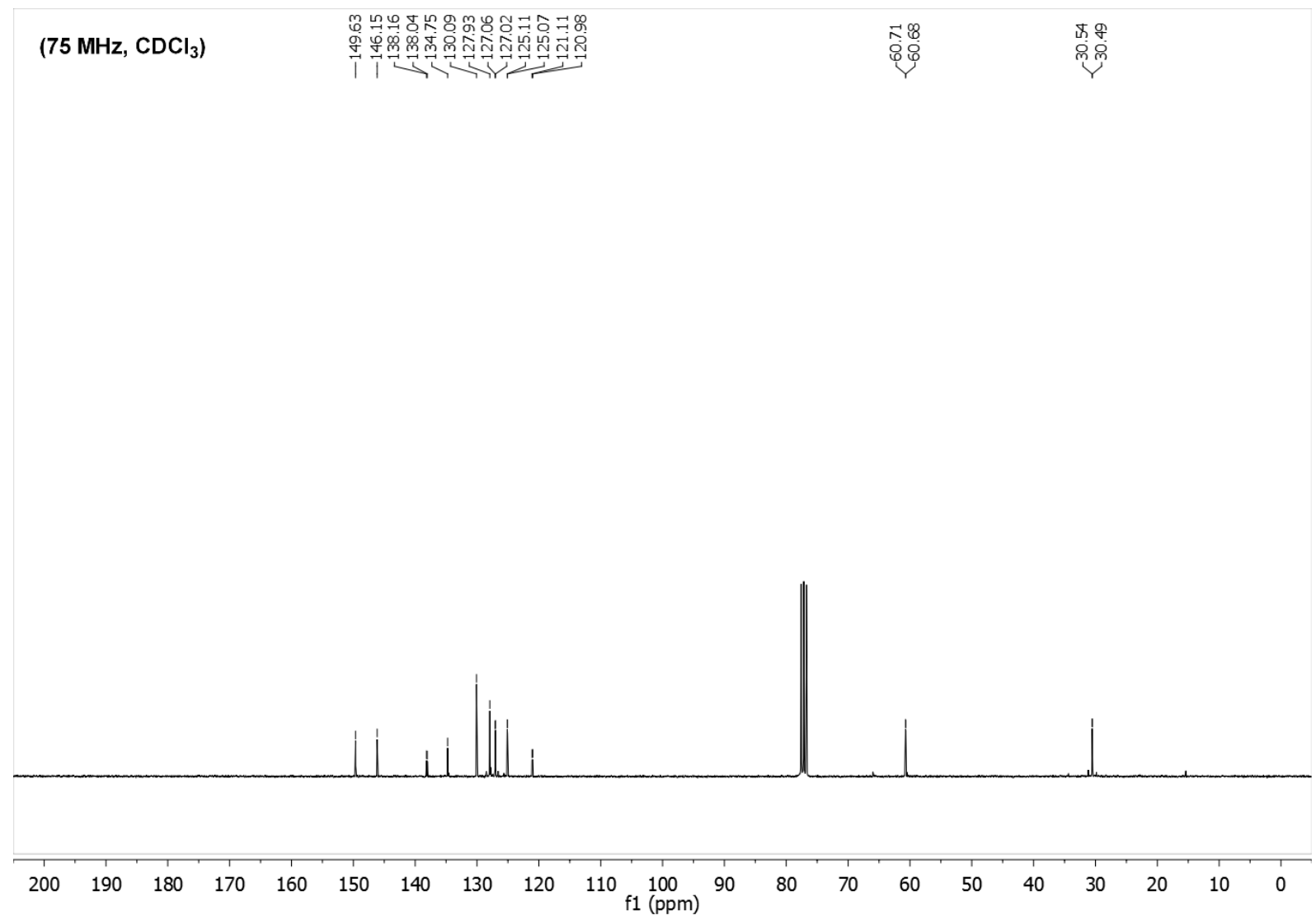

(282 MHz, $\mathrm{CDCl}_{3}$ )
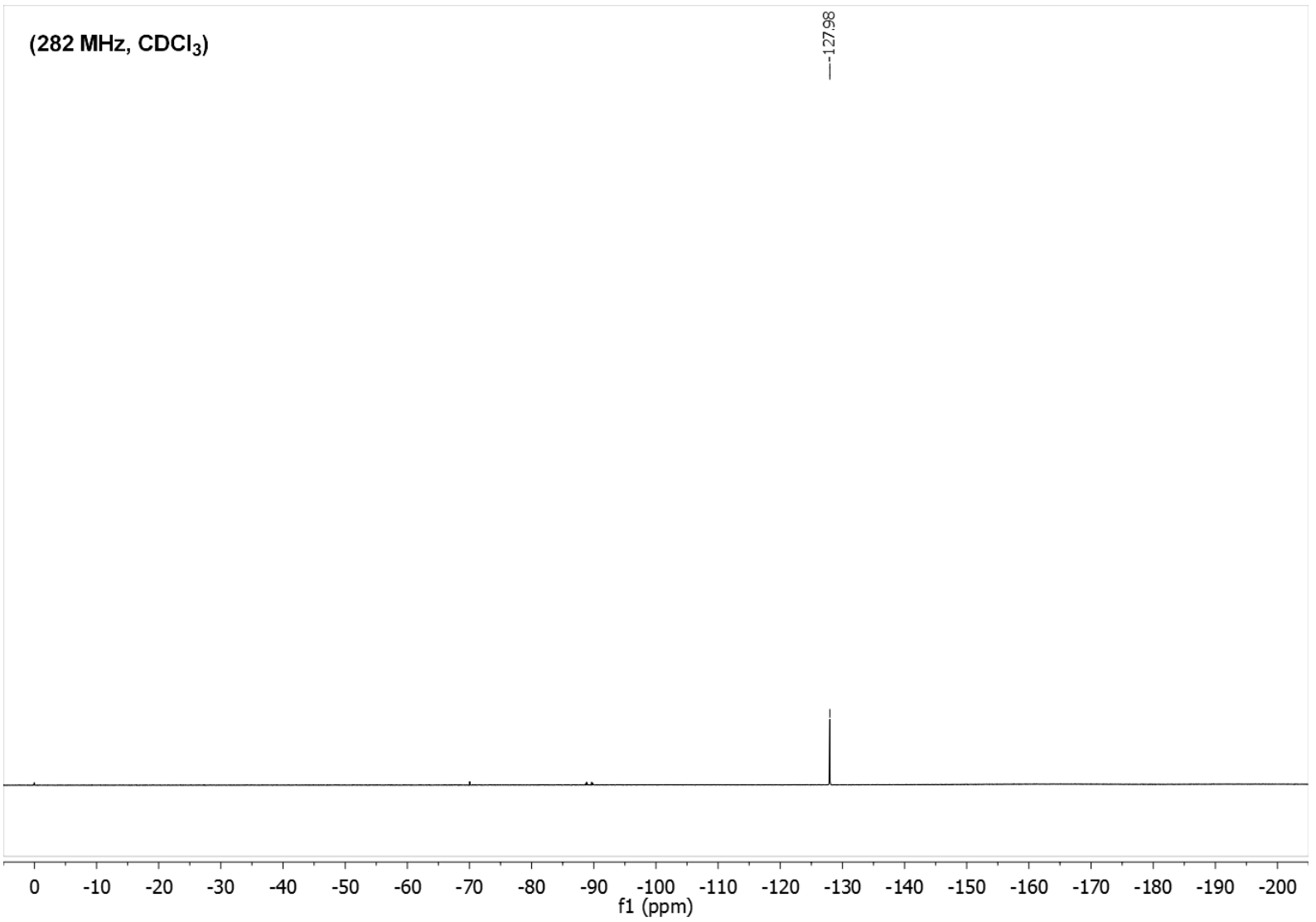


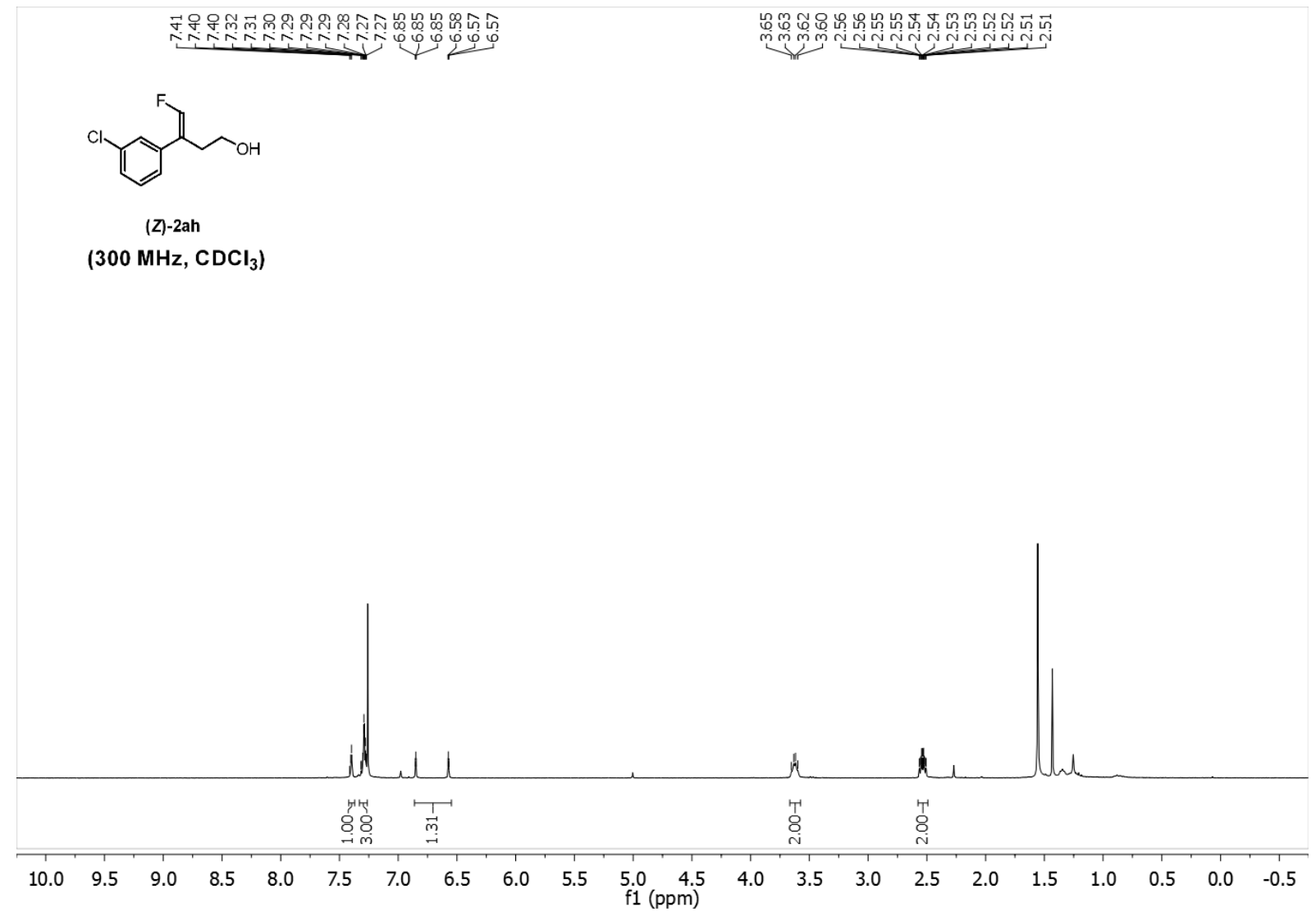

(75 $\mathrm{MHz}, \mathrm{CDCl}_{3}$ )

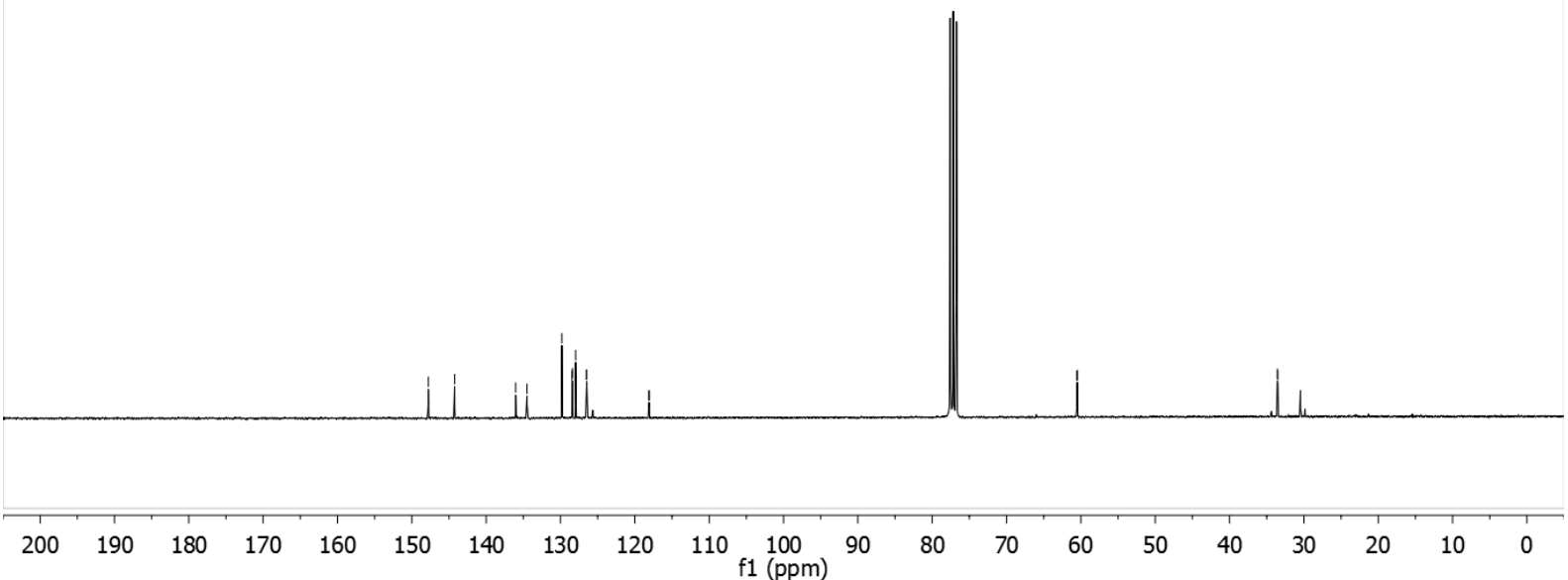


(282 $\mathrm{MHz}, \mathrm{CDCl}_{3}$ )

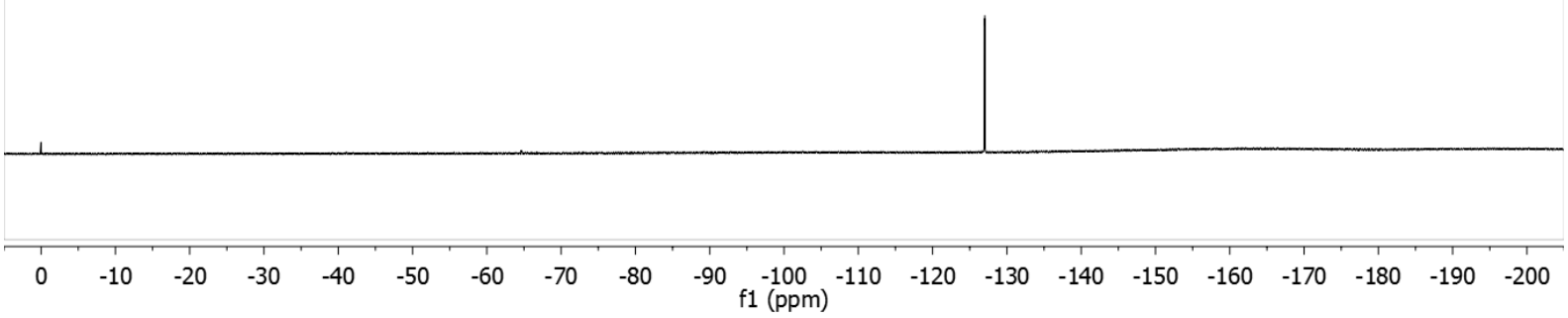

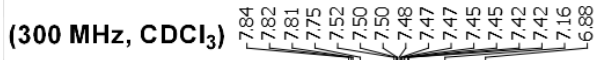

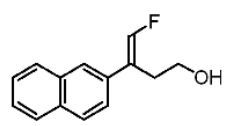

(E)-2ai

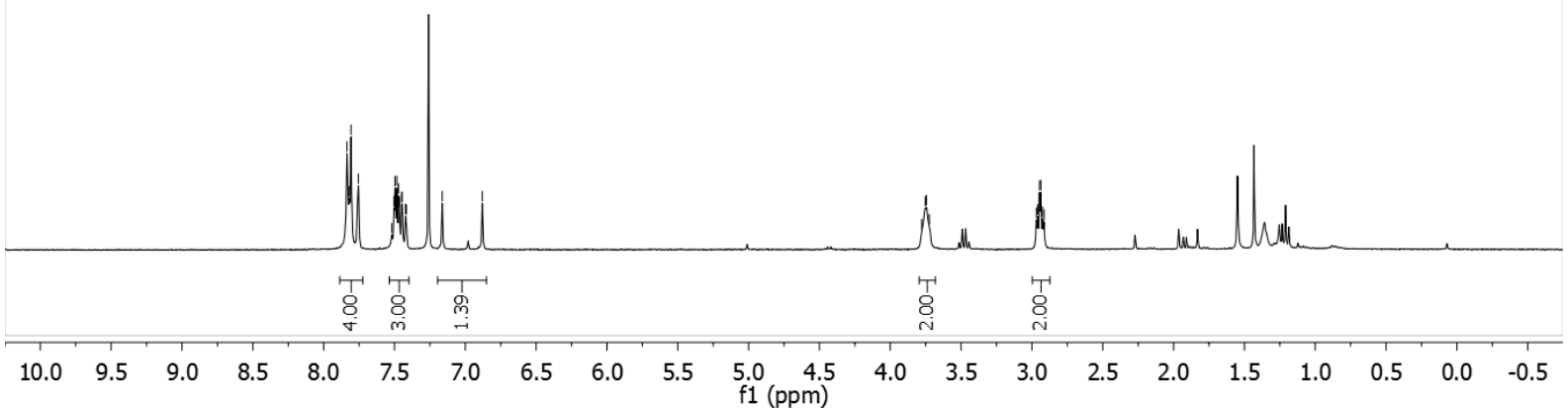




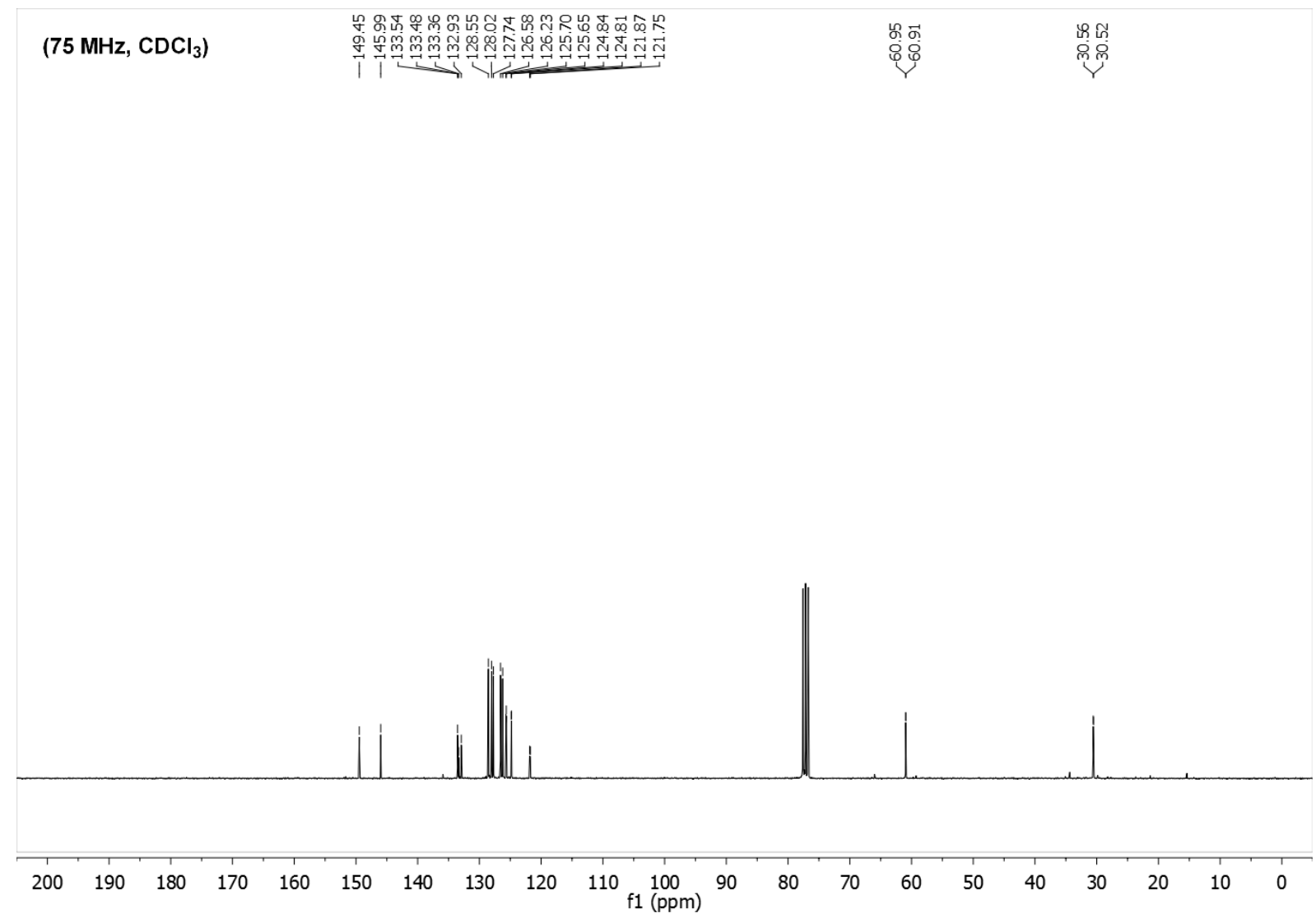

$\left(282 \mathrm{MHz}, \mathrm{CDCl}_{3}\right)$ 

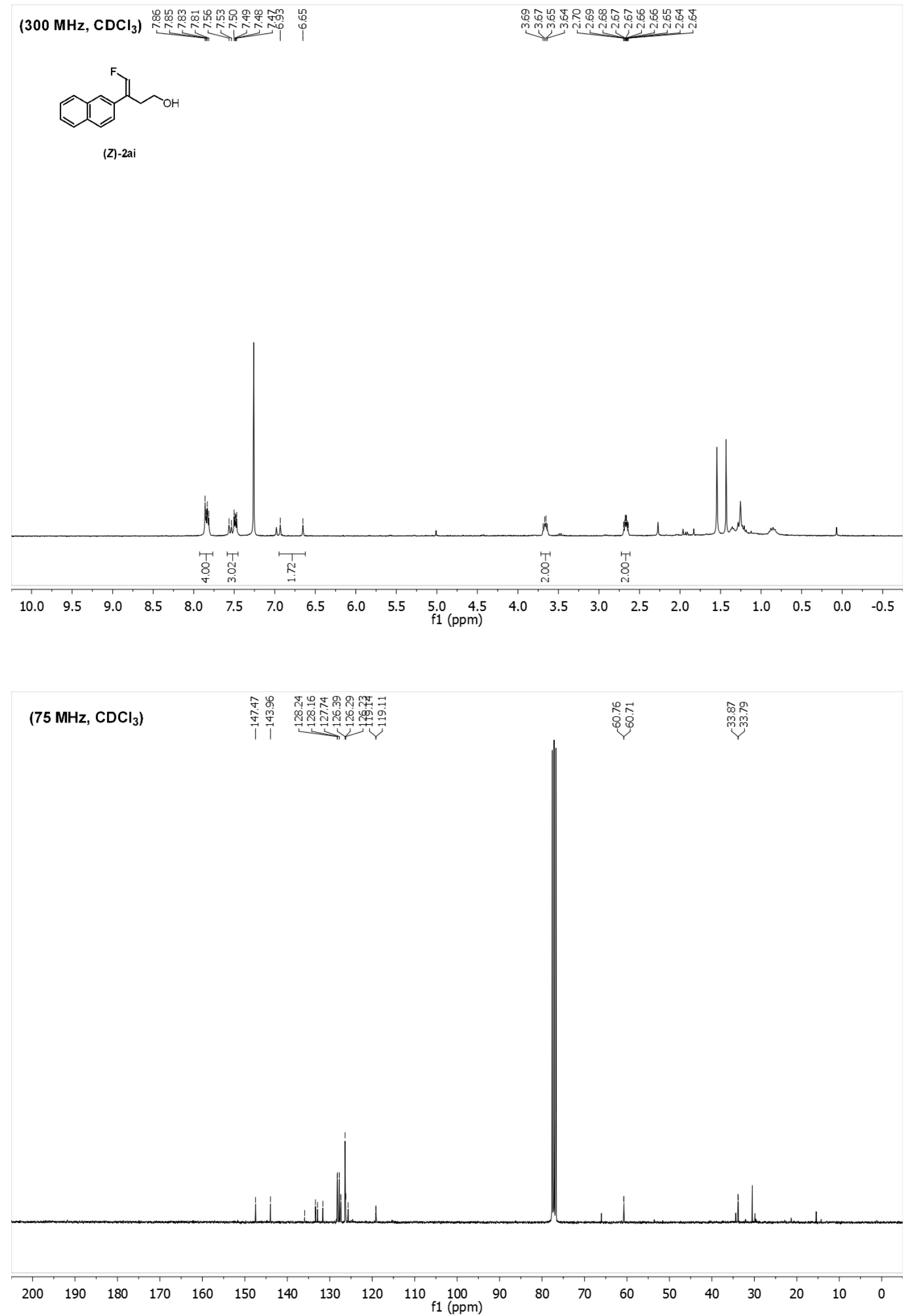
(282 $\mathrm{MHz}, \mathrm{CDCl}_{3}$ )

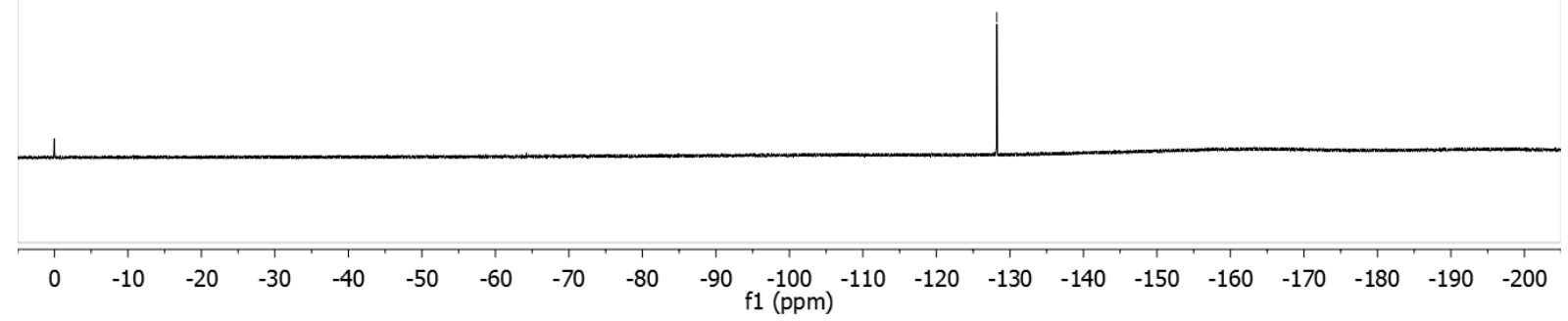

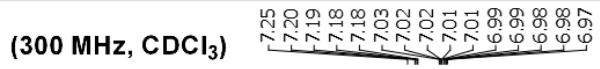

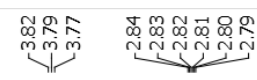

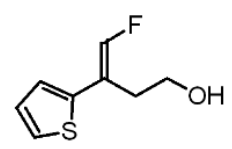

(E)-2aj

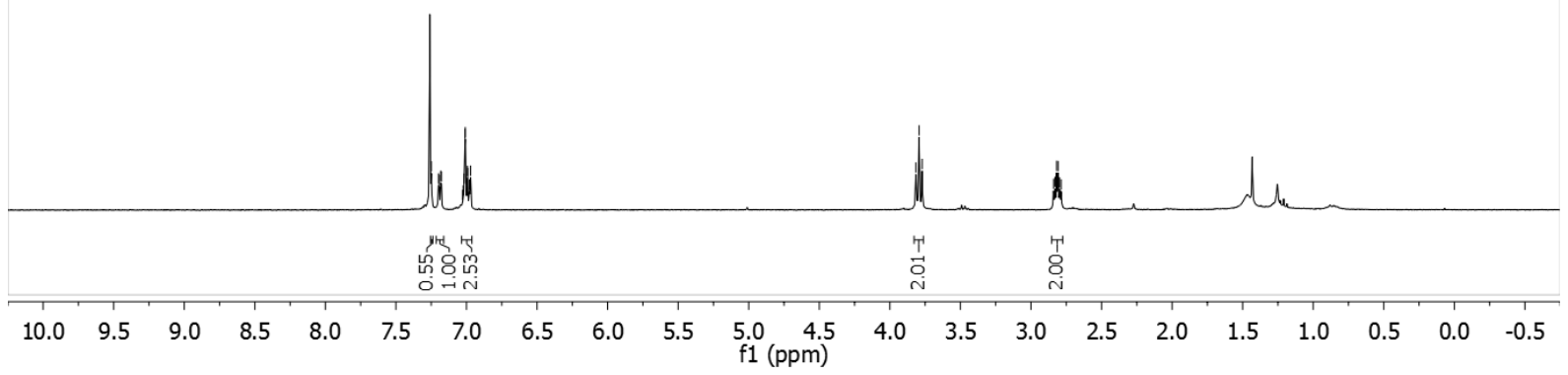




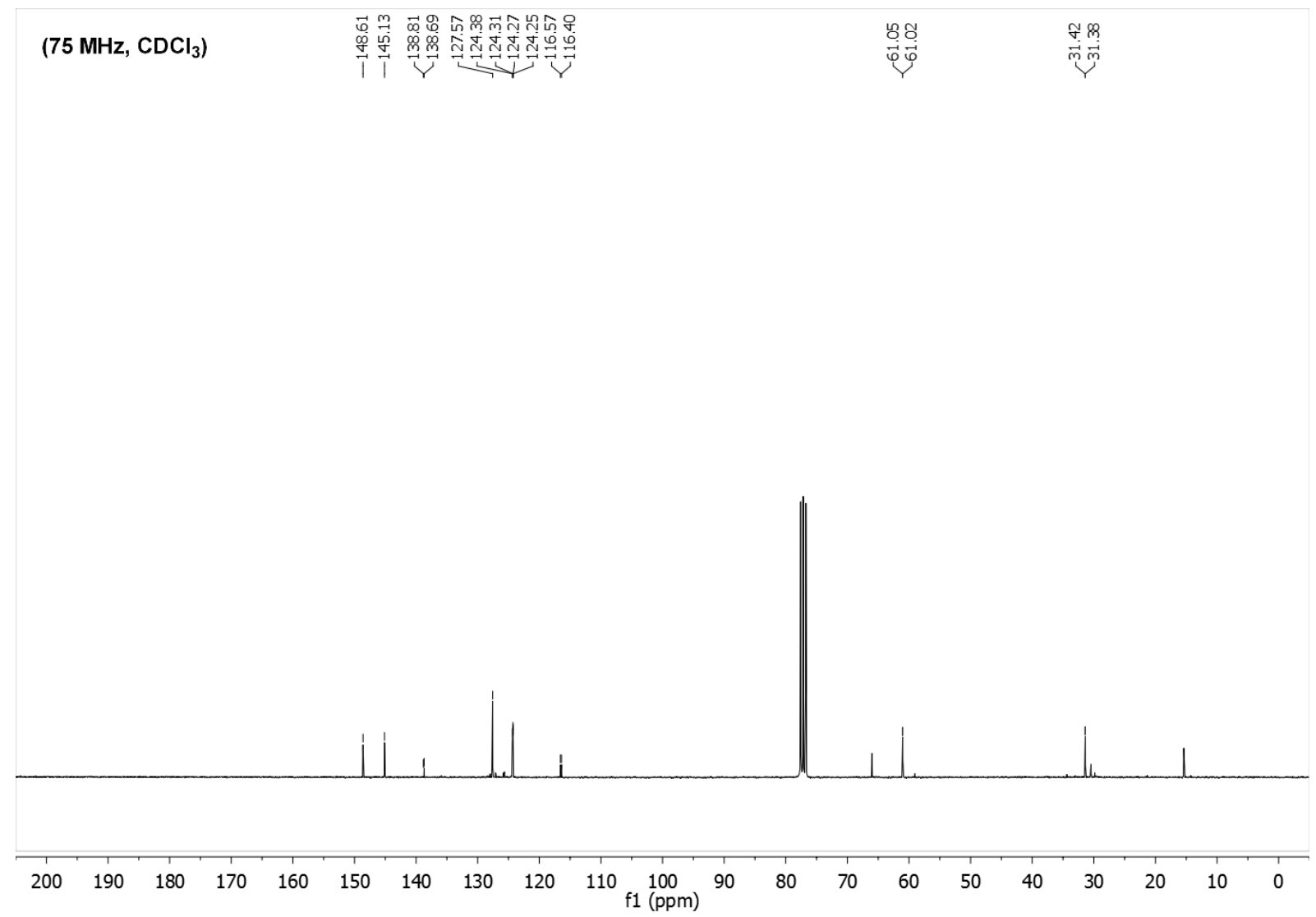

(282 MHz, $\mathrm{CDCl}_{3}$ )

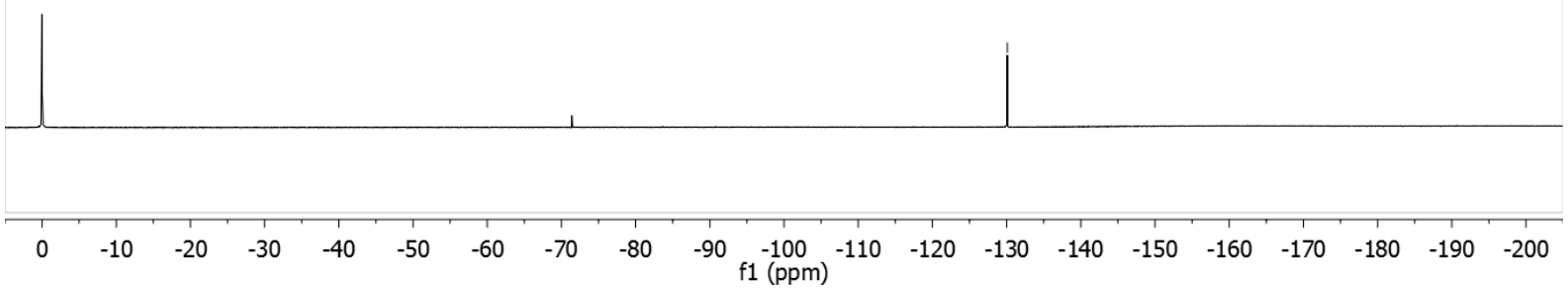




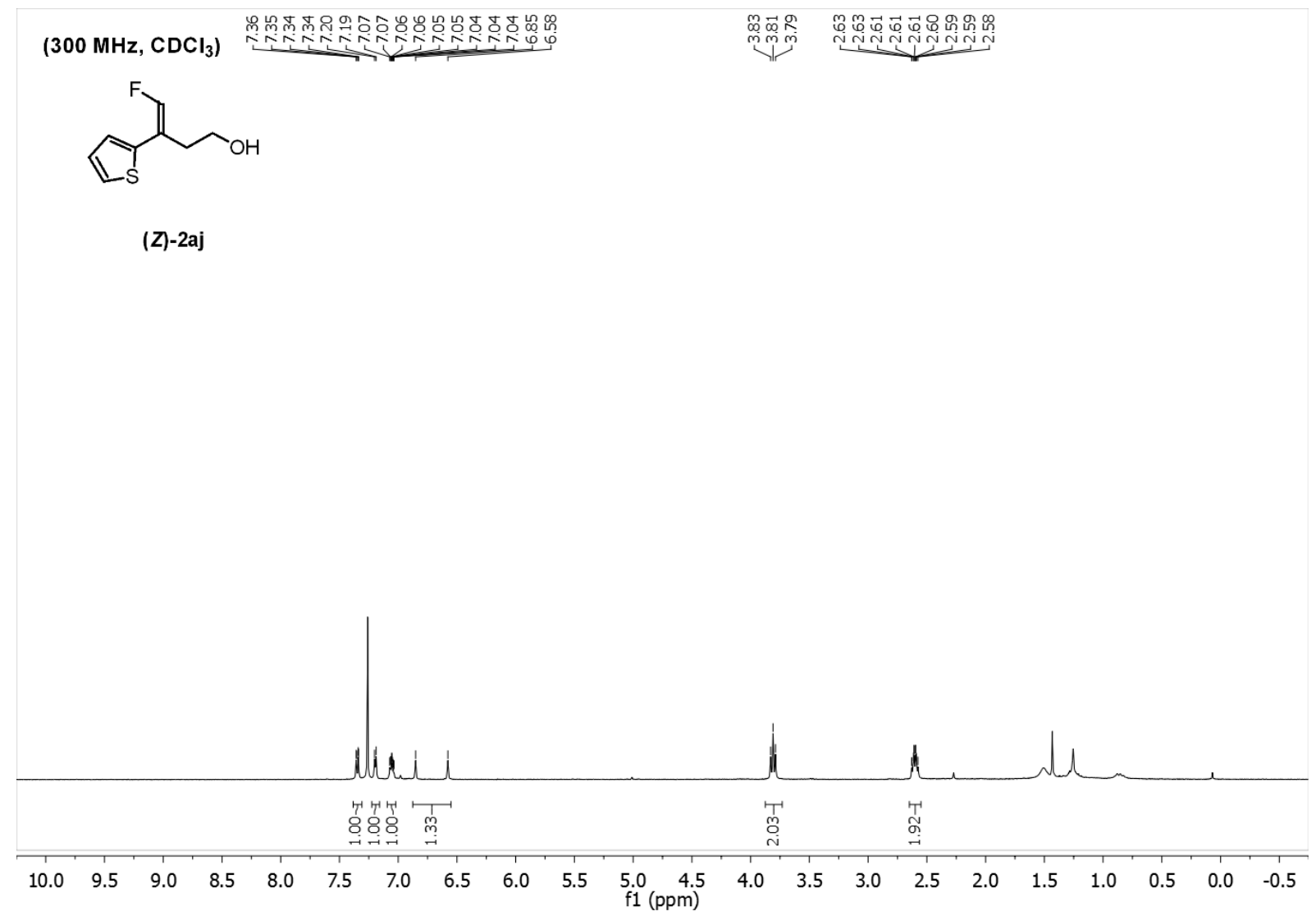

(75 MHz, $\mathrm{CDCl}_{3}$ )

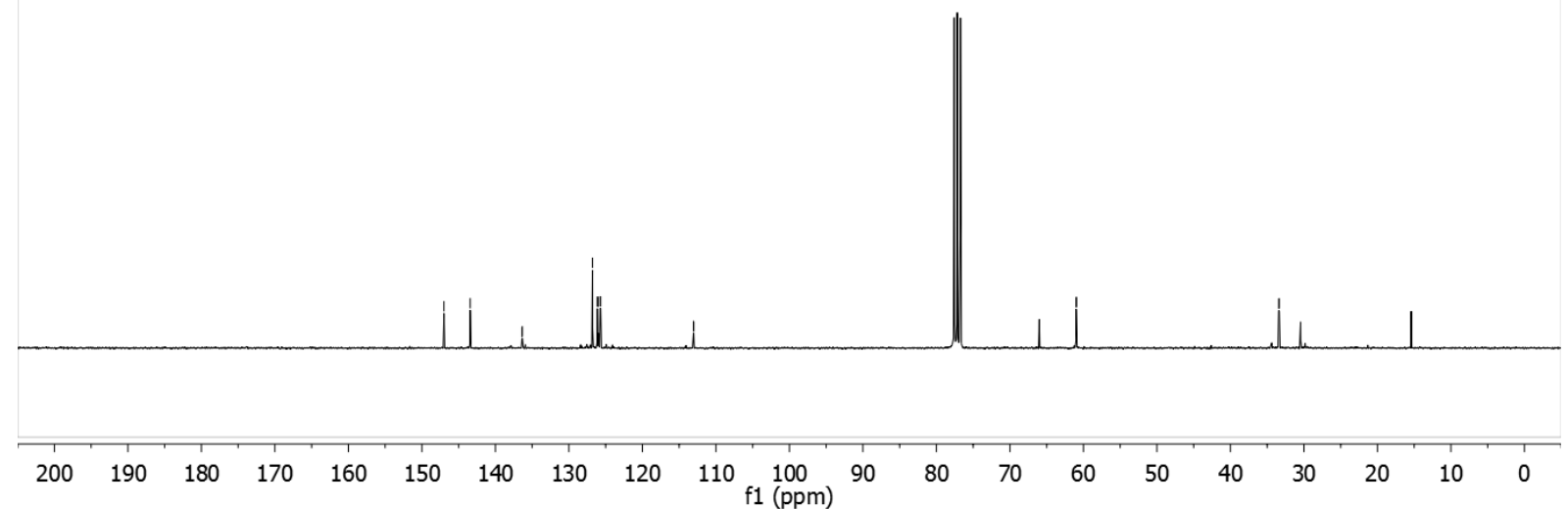


(282 $\mathrm{MHz}, \mathrm{CDCl}_{3}$ )

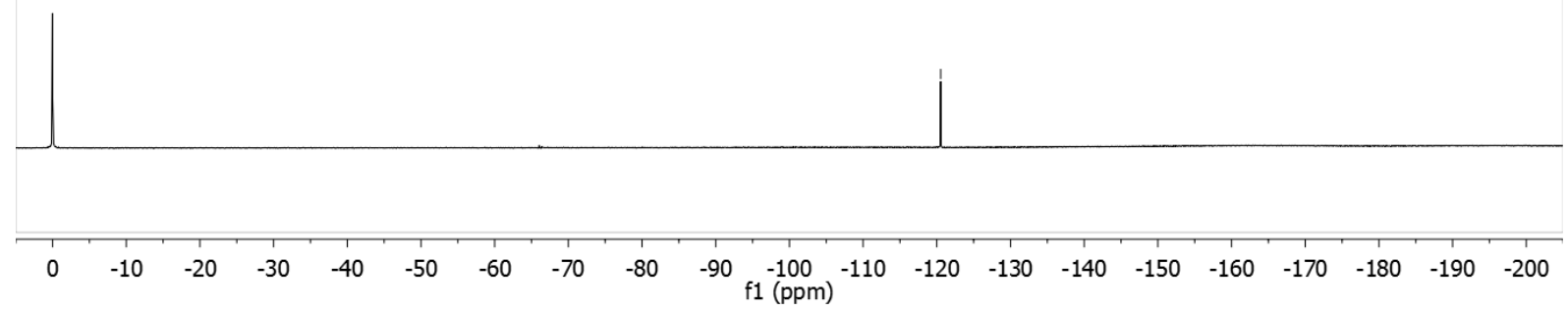

(300 MHz, $\left.\mathrm{CDCl}_{3}\right)$<smiles>CC(CO)C(=CF)c1ccccc1</smiles>

$(E)-2 a k$

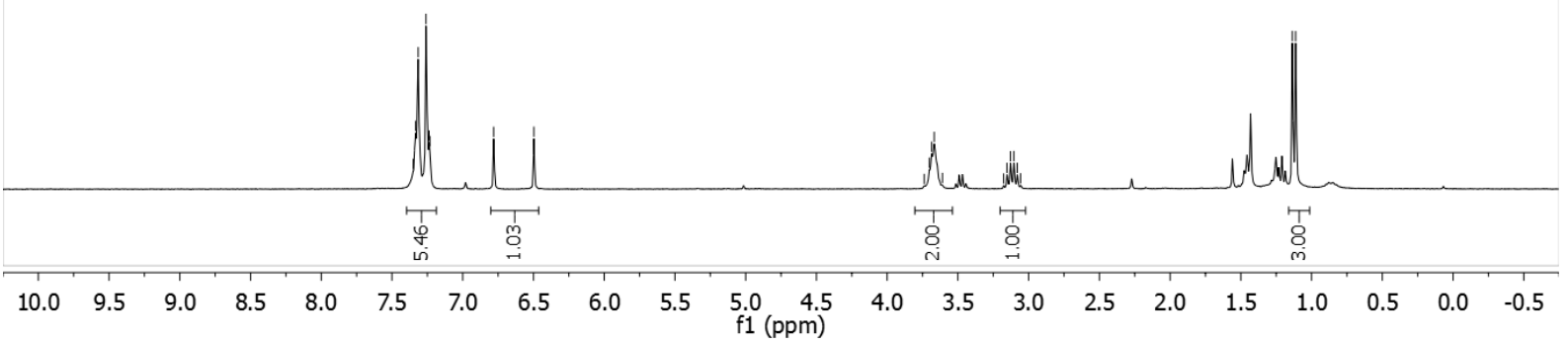




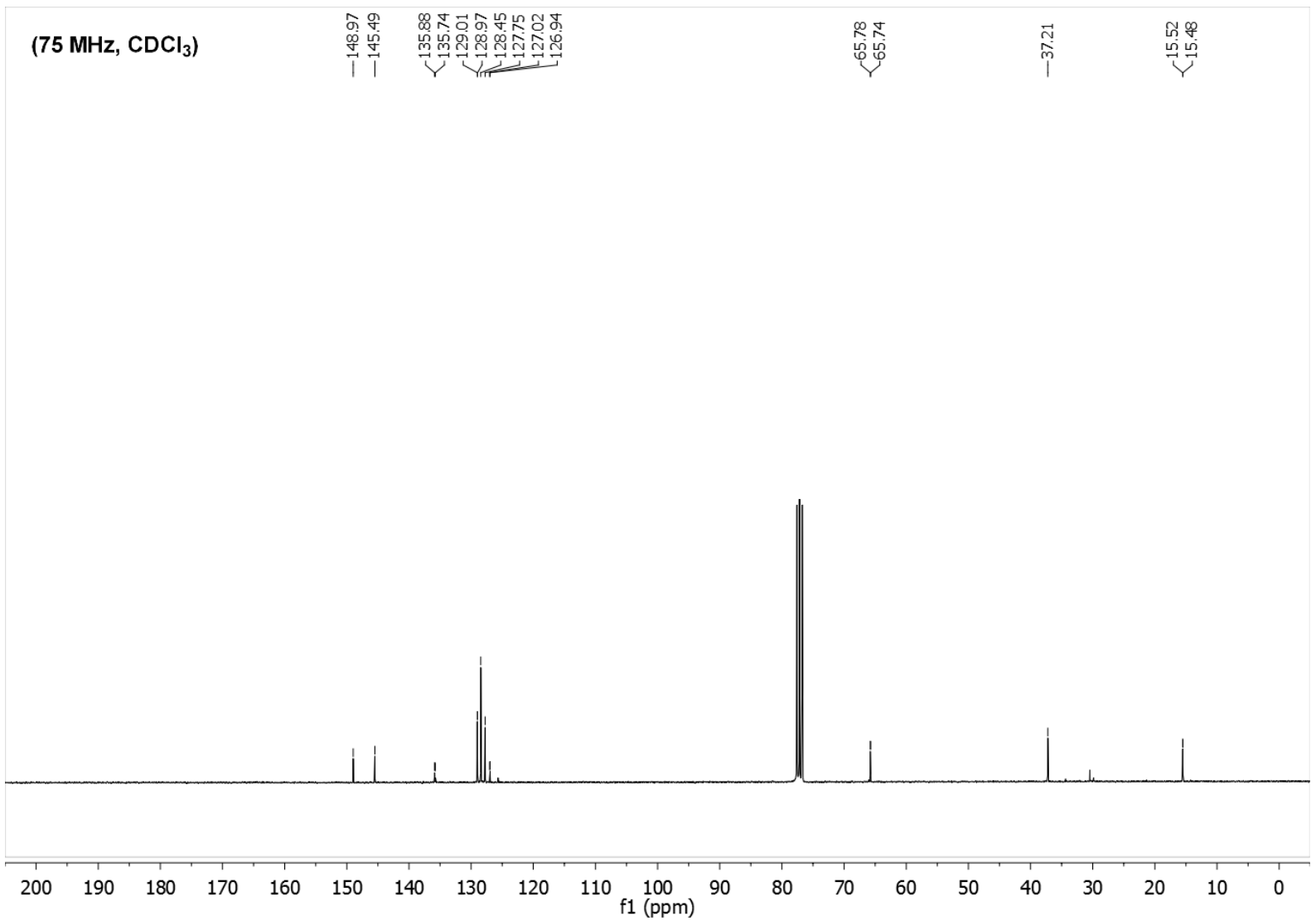

(282 $\mathrm{MHz}, \mathrm{CDCl}_{3}$ )

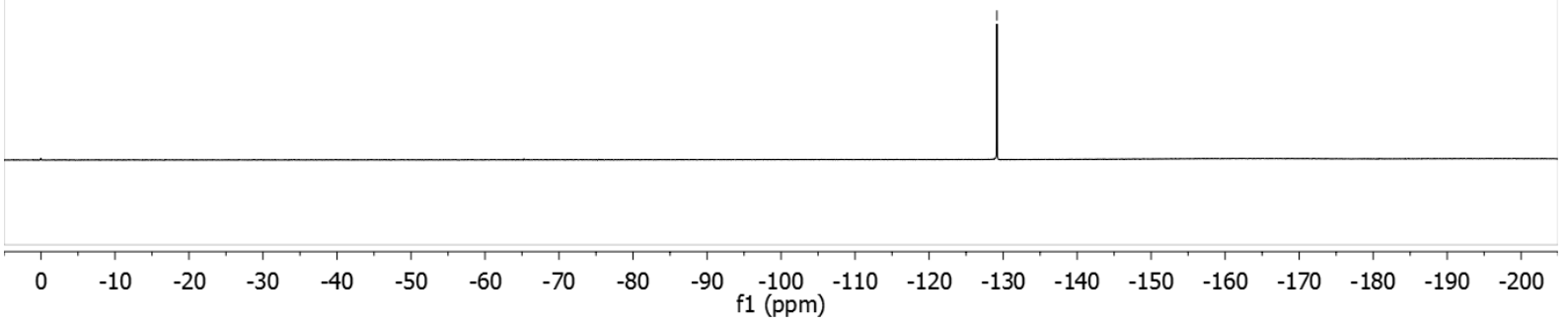




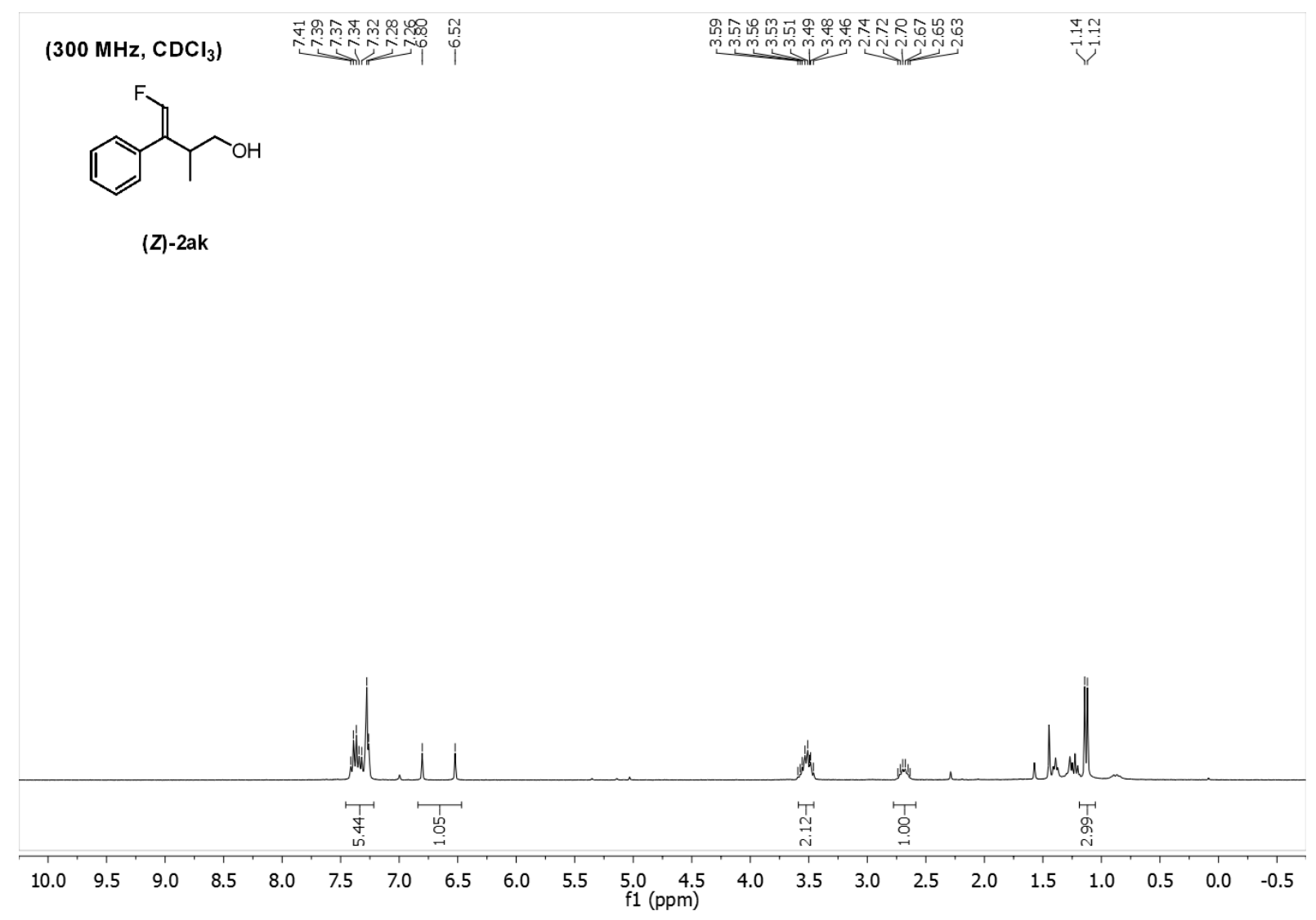

(75 MHz, $\mathrm{CDCl}_{3}$ )

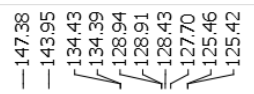

Yิ)

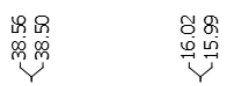

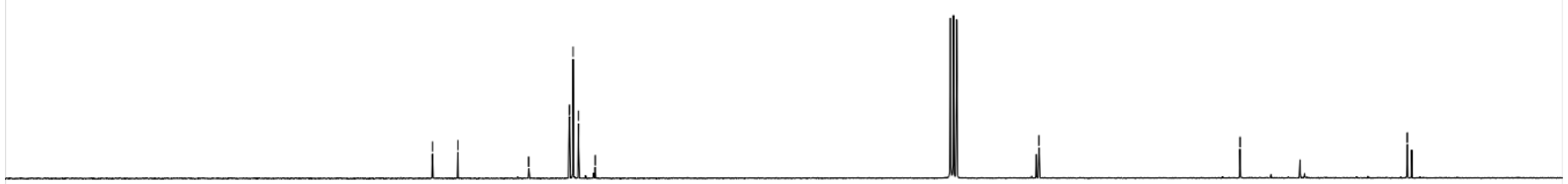


(282 MHz, $\mathrm{CDCl}_{3}$ )

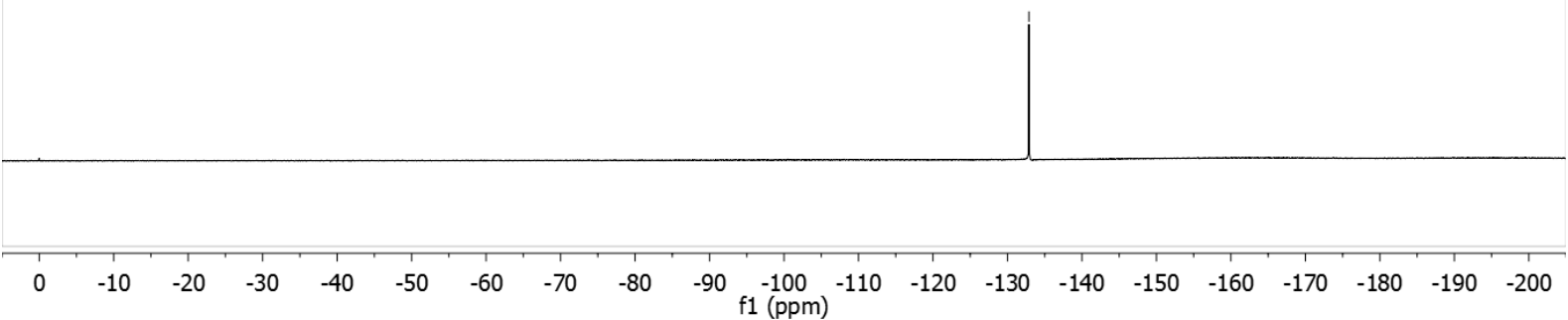

